\title{
Winter Fuels \\ Report
}

\section{Week Ending: February 18, 1994}

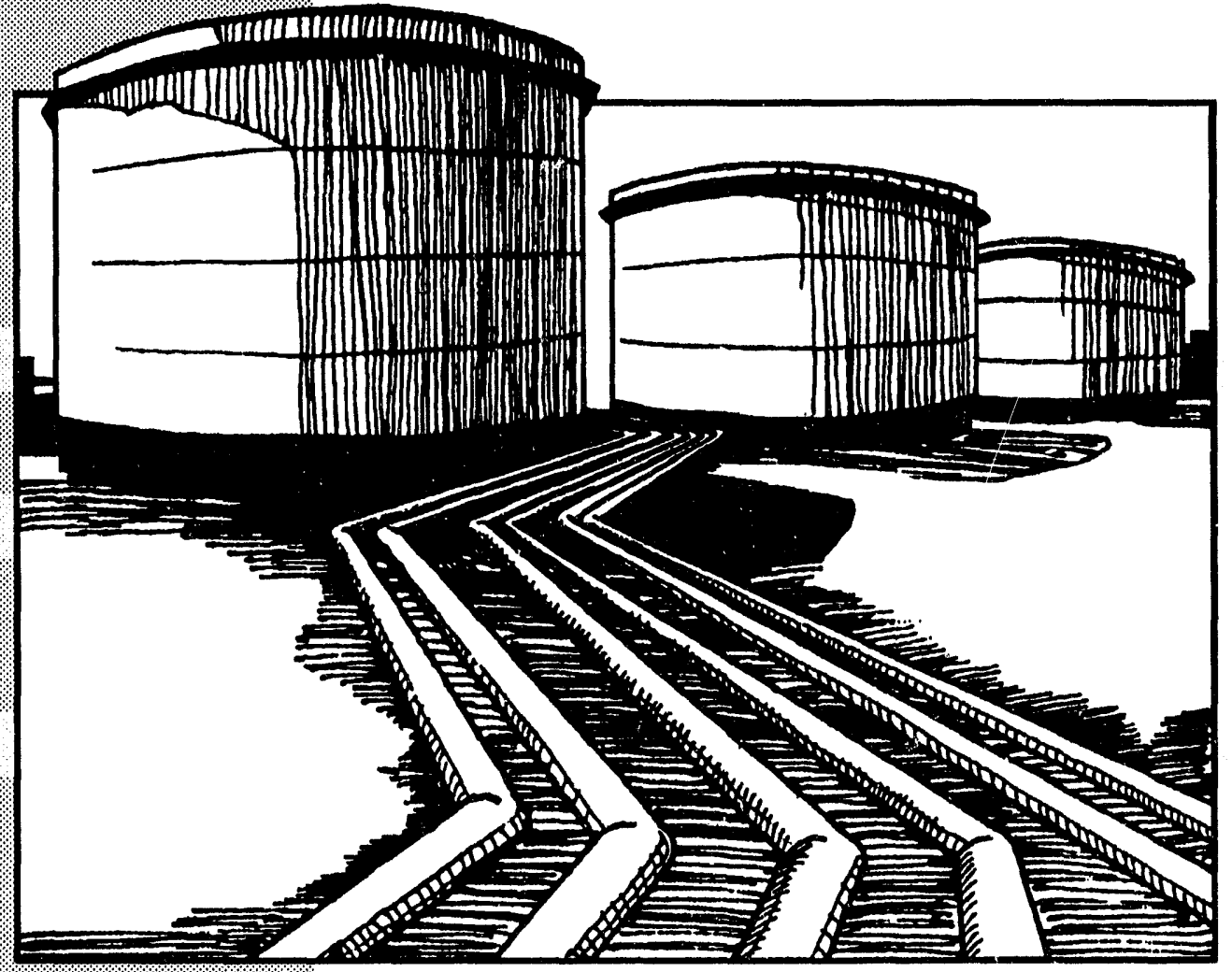

\section{MESTER}

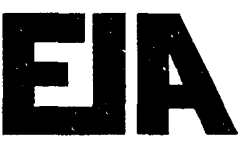

Energy Information Administration ds 
This publication is available free of charge from the National Energy Information Center at the following address:

National Energy Information Center, EI-231

Telecommunications Device for the

Energy Information Administration

Hearing Impaired Only: (202) 586-1181

Forrestal Building, Room 1F-048

9:00 a.m. to 5:00 p.m., eastern time, M-F

Washington, DC 20585

(202) $586-8800$

We thank the following for the use of their photographs and illustrations in this report.

Champlin Petroleum Co., page ix (courtesy of the American Petroleum Institute).

Texaco Inc., page 1 (courtesy of Texaco Inc.).

Cities Service Co., page 11 (courtesy of the American Petroleum Institute).

Atlantic Richfield Co., page 21 (courtesy of the American Petroleum Institute).

Chevron USA Inc., page 33 (courtesy of the American Petroleum Institute).

Amerada Hess Corp., page 51 (courtesy of Amerada Hess Corp.).

Texaco Inc., page 57 (courtesy of Texaco Inc.).

Standard Oil Co., page 61 (courtesy of the American Petroleum Institute).

Released for printing: February 24, 1994

Cover: An artist's rendering of bulk terminal storage tanks. 
DOE/EIA-0538(93/94-20)

Distribution Category UC-98

\title{
Winter Fuels Report
}

\author{
Week Ending: \\ February 18, 1994
}

\author{
Energy Information Administration \\ Office of Oil and Gas \\ U.S. Department of Energy \\ Washington, DC 20585
}

This report was prepared by the Energy Information Administration, the independent statistical and analytical agency within the Department of Energy. The information contained herein should not be construed as advocating or reflecting any policy position of the Department of Energy or any other organization. 


\section{Electronic Publishing System (EPUB)}

\section{User Instructions}

EPUB is an electronic publishing system maintained by the Energy Information Administration of the U.S. Department of Energy. EPUB allows the general public to electronically access selected energy data from many of EIA's statistical reports. The system is a menu-driven, bulletin board type system with extensive online help capabilities that can be accessed free of charge 24 hours a day by using a terminal or PC with an asynchronous modem. (EPUB will be taken down briefly at nidnight for backup.)

\section{CONFIGURING YOUR PC SOFTWARE}

PC users must provide the following information to their communications software in order to successfully access the EPUB system. Consult your communications software documentation for information on how to correctly configure your software.

Communication Parameters:

BAUD RATE: $300-2400$ bps

DATA BITS: 8

STOP BITS: 1

PARITY: NONE

DUPLEX: FULL

TERMINAL TYPE: examples: ANSI, ANSI-BBS, VT100

\section{ACCESS PHONE NUMBER}

Once your communications software and/or hardware has been configured, you can access EPUB by dialing (202) 586-2557.

\section{USING EPUB}

When a connection to the system has been made, some users may find that the menu-driven instructions and the online help capabilities will provide enough information to effectively use EPUB. If needed, more extensive information may be found in the EPUB Users Guide, which is available online from the EPUB system or from:

National Energy Information Center, EI-231

Energy Information Administration

Forrestal Building, Room 1F-048

Washington, DC 20585

(202) $586-8800$

Hours: 9:00 a.m. to 5:00 p.m. Eastern Time, Monday through Friday

Telecommunications device for the hearing-imparied only:

(202)586-1181. Hours 9:00 a.m. to 5:00 p.m. Eastern Time, Monday through Friday.

\section{EPUB ASSISTANCE:}

For communications or technical assistance, call (202) 586-8959, 8:00 a.m. to 5:00 p.m. Eastern Time, Monday through Friday.

For questions about the content of EPUB reports, call (202) 586-8800, 8:00 a.m. to 5:00 p.m. Eastern Time, Monday through Friday.

\section{EPUB PROVIDES SELECTED DATA FROM THE FOLLOWING EIA PUBLICATIONS:}

Heating fuel data, (April through September) updated the 2nd week of the month

Oxygenate data, updated approximately 15 working days after the end of the report month

Weekly Petroleum Status Report, updated on Wednesdays (Thursday in event of a holiday) at 9:00 a.m.

Petroleum Supply Monthly, updated on the 20th of the month

Petroleum Marketing Monthly, updated on the 20th of the month

Winter Fuels Report, propane inventory data updated Wednesdays at 5:00 p.m. All other data updated on Thursdays (Friday in event of a holiday) at 5:00 p.m. (October through March)

Natural Gas Monthly, updated on the 20th of the month

Weekly Coal Production, updated on Fridays at 5:00 p.m.

Quarterly Coal Report, updated 60 days after the end of the quarter

Electric Power Monthly, updated on the 1st of the month

Monthly Energy Review, updated the last week of the month

Short Term Energy Outlook, updated 60 days after the end of the quarter 


\section{Contacts}

The Winter Fuels Report is prepared by the Office of Oil and Gas, Energy Information Administration. General information about this document may be obtained from Jimmie L. Petersen (202) 586-6401, Director of the Office of Oil and Gas or Charles C. Heath (202) 586-6860, Director of the Petroleum Supply Division. Specific questions can be directed to:

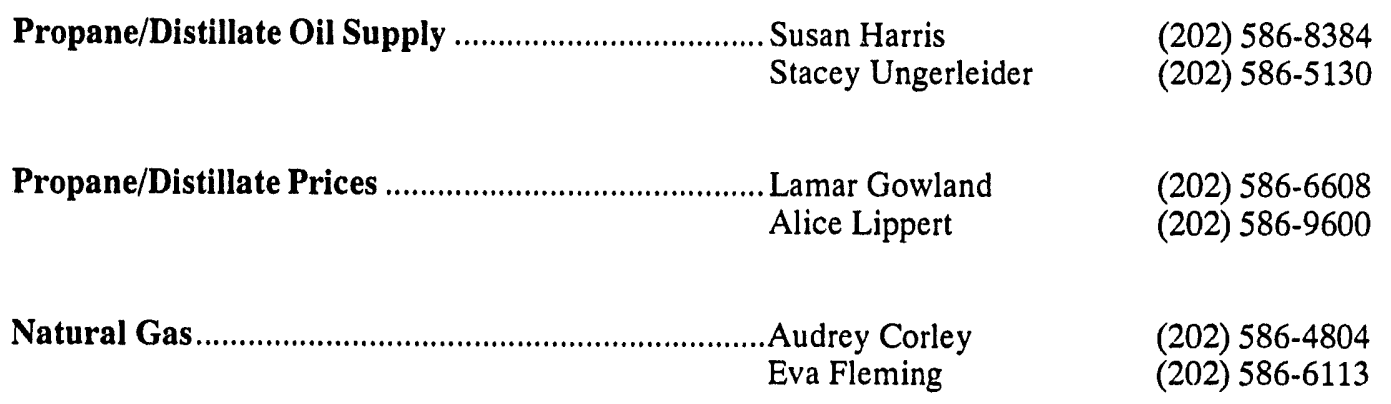

Copies of this report are available to the press through the Department of Energy's Public Inquires, Room 1E-206, Forrestal Building, Washington, DC, (202) 586-5575 and to the public through the National Energy Information Center, Room 1F-048, Forrestal Building, Washington, DC, (202) 586-8800. 


\section{Preface}

The Winter Fuels Report is intended to provide concise, timely information to the industry, the press, policymakers, consumers,

analysts, and State and local governments on the following topics:

distillate fuel oil net production, imports and stocks on a U.S. level and for all Petroleum Administration for Defense Districts (PADD) and product supplied on a U.S. level;

propane net production, imports and stocks on a U.S. level and for PADD's I, II, and III;

natural gas supply and disposition and underground storage for the U.S. and consumption for all PADD's; as well as selected National average prices.

residential and wholesale pricing data for heating oil and propane for those States participating in the joint Energy Information Administration (EIA)/State Heating Oil and Propane Program;

crude oil and petroleum price comparisons for the U.S. and selected cities; and

a 6-10 Day, 30-Day, and 90-Day outlook for temperature and precipitation and U.S. total heating degree-days by city.

The distillate fuel oil and propane supply data are collected and published weekly. The data are based on company submissions for the week ending 7:00 a.m. for the preceding Friday. Weekly data for distillate fuel oil are also published in the Weekly Petroleum Status Report. Monthly data for distillate fuel oil and propane are published in the Petroleum Supply Monthly.

The residential pricing information is collected by the EIA and the State Energy Offices on a semimonthly basis for the EIA/State Heating Oil and Propane Program. The wholesale price comparison data are collected daily and are published weekly. Residential heating fuel prices are derived from price quotes for home delivery of No. 2 fuel oil and propane. As such, they reflect prices in effect on the dates shown. Wholesale heating oil and propane prices are estimates using a sample of terminal quotes to represent avcrage State prices on the dates given. The Computer Petroleum Corporation, Inc., defines these prices to be prices f.o.b. terminal, cxcluding taxes, discounts, and hauling allowances. The crude oil and petroleum product prices are from various industries sources as referenced on each table.

The natural gas data are collected and published monthly in the Natural Gas Monthly.

This report will be published weekly by the EIA starting the second week in October 1993 and will continue until the second week in April 1994. The data will also be available electronically after 5:00 p.m. on Wednesday and Thursday during the heating scason through the EIA Electronic Publication System (EPUB). See page ii for details. 


\section{Contents}

Highlights

Tables

Distillate Fuel Oil

1. Monthly and Weekly Net Production, Imports, and Stocks of Distillate Fuel Oil by Petroleum

Administration for Defense District (PADD) and Product Supplied for the United States

Propane

2. Monthly and Weekly Net Production, Imports, and Stocks of Propane/Propylene by Petroleum

Administration for Defense Districts (PADD) I, II, and III

Natural Gas

3. Supply and Disposition of Dry Natural Gas in the United States.

4. Underground Natural Gas Storage in the United States (All Operators)

5. Natural Gas Consumption by Petroleum Administration for Defense District (PADD)

6. Selected National Average Natural Gas Prices in the United States.

Prices

7. Residential Heating Oil Prices by Region and State

8. Residential Propane Prices by Region and State

9. Wholesale Heating Oil Prices by Region and State

10. Wholesale Propane Prices by Region and State

11. U.S. Crude Oil and Petroleum Product Prices

12. Petroleum Product Prices for Selected Cities

Weather Summary

Highlights 48

13. U.S. Total Heating Degree-Days by City .

\section{Illustrations}

F1. U.S. Distillate Fuel Oil Production

F2. U.S. Distillate Fuel Oil Imports

F3. U.S. Distillate Fuel Oil Stocks

F4. PADD I (East Coast) Distillate Fuel Oil Stocks

F5. PADD II (Midwest) Distillate Fuel Oil Stocks

F6. PADD III (Gulf Coast) Distillate Fuel Oil Stocks

F7. PADD IV (Rocky Mountain) Distillate Fuel Oil Stocks

F8. PADD V (West Coast) Distillate Fuel Oil Stocks

F9. U.S. Propane Production

F10. U.S. Propane Imports

F11. U.S. Propane Stocks

F12. PADD I (East Coast) Propane Stocks

F13. PADD II (Midwest) Propane Stocks ....

F14. PADD III (Gulf Coast) Propane Stocks

F15. Underground Natural Gas Storage in the United States

F16. Natural Gas Deliveries to Consumers in the United States

F17. Average Price of Natural Gas Delivered to Consumers in the United States...

F18. Average Price of Natural Gas in the United States

F19. Residential Heating Oil Prices, New England

F20. Residential Heating Oil Prices, Central Atlantic

F21. Residential Heating Oil Prices, Lower Atlantic

F22. Residential Heating Oil Prices, Midwest

F23. Residential Propane Prices, New England

F24. Residential Propane Prices, Central Atlantic

F25. Residential Propane Prices, Lower Atlantic

F26. Residential Propane Prices, Midwest

F27. Wholesale Heating Oil Prices, New England

F28. Wholesale Heating Oil Prices, Central Atlantic

F29. Wholesale Heating Oil Prices, Lower Atlantic

F30. Wholesale Heating Oil Prices, Midwest

F31. Wholesale Propane Prices, Central Atlantic

F32. Wholesale Propane Prices, Lower Atlantic

F33. Wholesale Propane Prices, Midwest

F34. 6-10 Day Temperature Outlook 
F35. 6-10 Day Precipitation Outlook

F36. 30 Day Temperature Outlook

F37. 90 Day Temperature Outlook.

\section{Appendices}

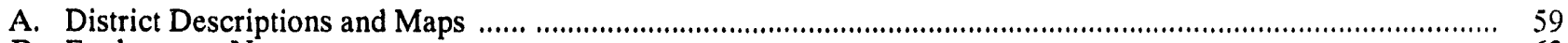

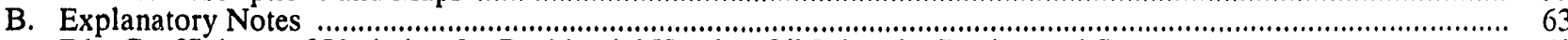

B1. Coefficients of Variation for Residential Heating Oil Prices by Region and State .................................. 68

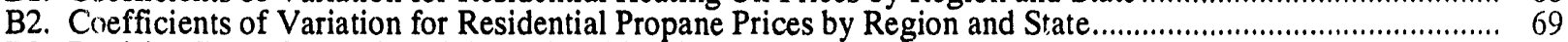

B3. Revision Rates for Residential Heating Oil Prices by Region and State ................................................. 70

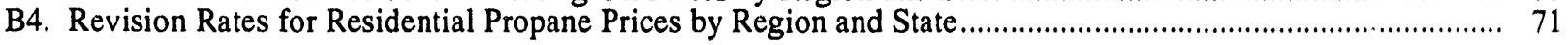

\section{Glossary}




\section{Highlights}

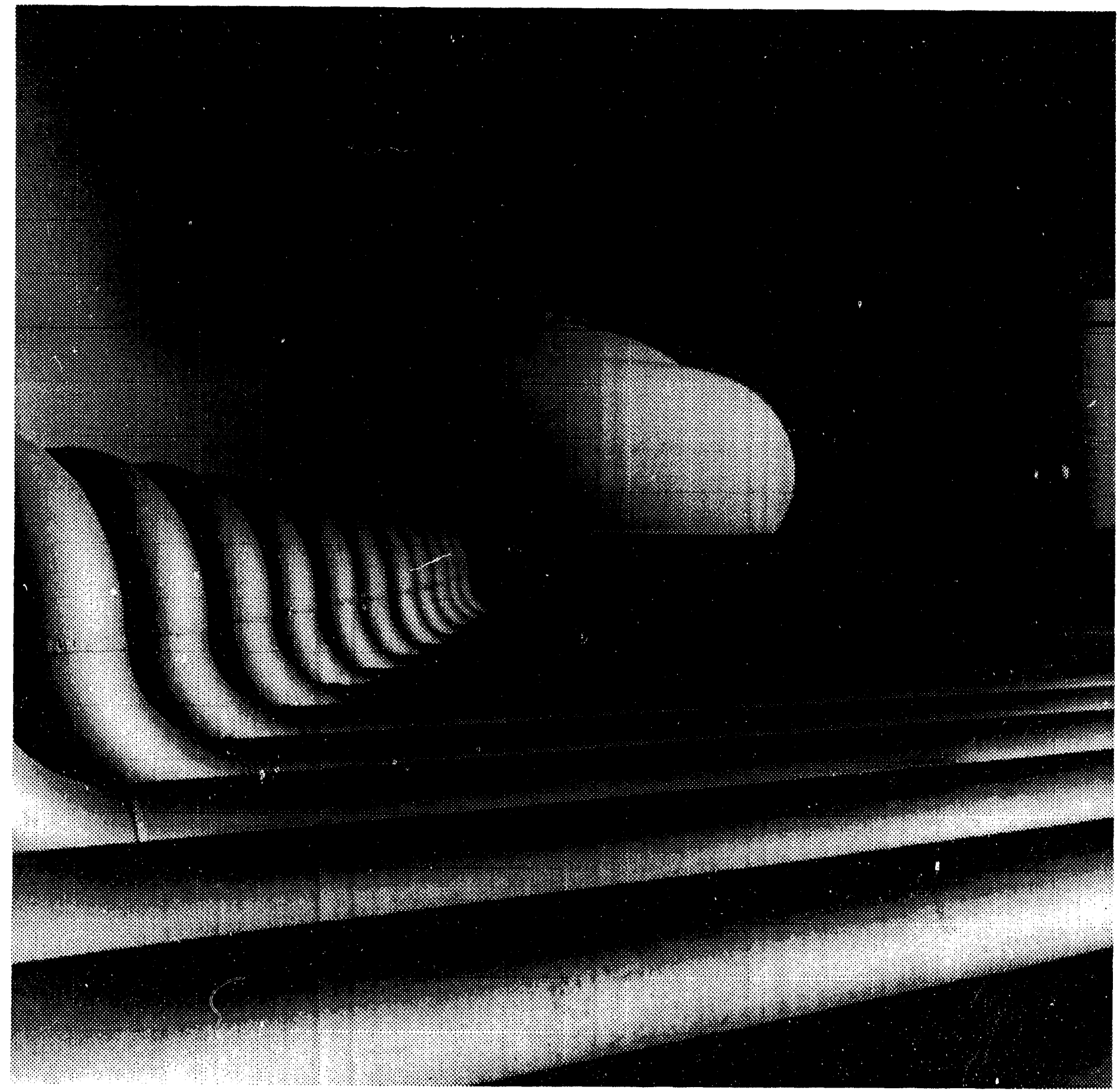

Liquefied petroleum gases are stored in pressurized tanks while other products are stored in conventional tanks. 


\section{Highlights}

\section{DISTILLATE FUEL OIL}

Distillate fuel oil stocks in New England (PADD IX) were down 1.9 MMB last week while PADD IY inventories gained 0.4 MMB. PADD I stocks were down 1.2 MMB over all, and are 9.7 MMB below normal for this time of year. Stocks were also down in PADD

III as suppliers worked to replenish secondary stocks, particularly in the Eastern part of the Nation.

Imports were a large part of this week's supply; at $0.3 \mathrm{MMBD}$ they reflected a price differential between New York Harbor and Rotterdam spot markets. This differential is expected to pull heating fuel into the Northeastern part of the country at very high levels over the next two weeks. Harbors are open, and except for continued barge travel limitation on the Hudson, water bourne supplies can move readily.

Table H1. Distillate Fuel Oll (Thousand Barrels per Day, Except Where Noted)

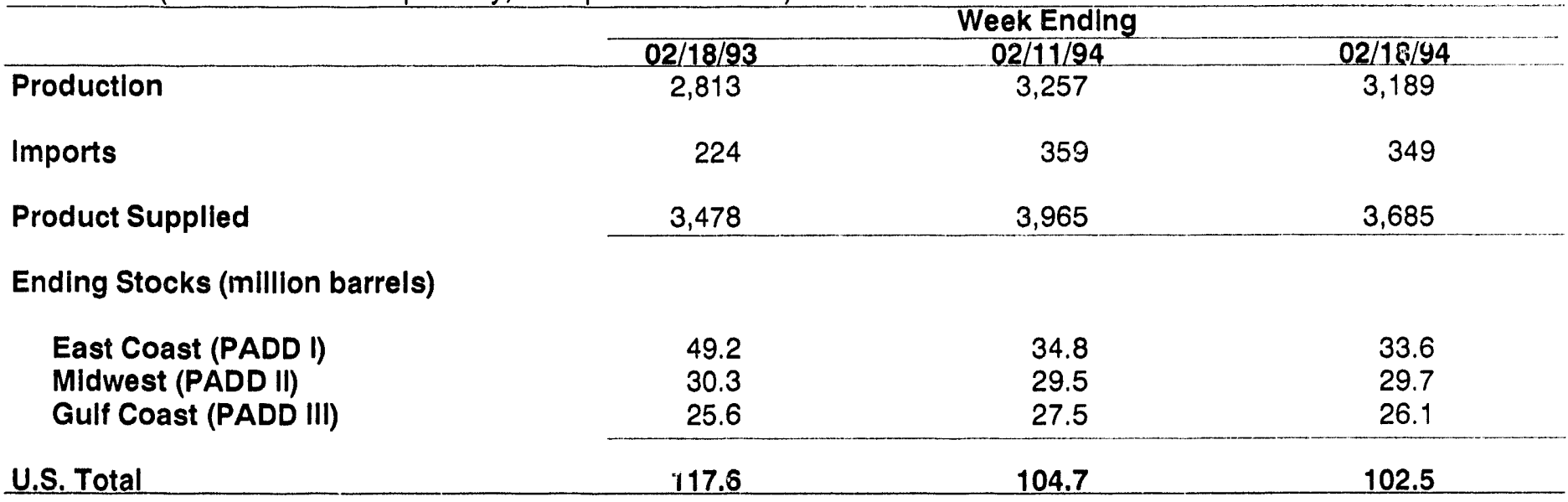

Source: Energy Information Administration (E|A), Weekly and Monthly Petroleum Supply Reporting Systems.

\section{PROPANE}

The U.S. propane inventory level recorded stronger-than-normal declines last week. The Nation's supply of propane for the week ending February 18, 1994, was 24.8 million barrels (MMB). This level was $2.7 \mathrm{MMB}$ lower than the inventory level for the week ending February 11, 1994.

During the week ending February 18, 1994, inventory levels increased in the East Coast but decreased in the Midwest and Gulf Coast. The East Coast stock level rose by 0.1 MMB. In the Midwest, levels dropped by $0.8 \mathrm{MMB}$ while in the Gulf Coast, which is the largest propane producing as well as consuming section of the Nation, the inventory level decreased by $1.9 \mathrm{MMB}$.

Despite a warming trend over portions of the Nation last week, Gulf Coast and Midwest inventories of propane continued to exhibit strong declines as pipeline shippers and distributors took advantage of the lull in the weather and shifted propane from primary storage to local distribution centers. The Energy Information Administration continues to recommend close monitoring of propane slock levels, especially if the weather turns colder than normal. 
Table H2. Propane Stocks by Petroleum Administration for Defense Districts (PADD) I, II, and III (Thousand Barrels)

\begin{tabular}{lcccccccc}
\hline PAD Districts & January & February & \multicolumn{7}{c}{ Week Ending } \\
\cline { 6 - 9 } & 1993 & 1993 & $\mathbf{0 1 / 1 4 / 9 4}$ & $\mathbf{0 1 / 2 1 / 9 4}$ & $\mathbf{0 1 / 2 8 / 9 4}$ & $\mathbf{0 2 / 0 4 / 9 4}$ & $\mathbf{0 2 / 1 1 / 9 4}$ & $02 / 18 / 94$ \\
\hline East Coast (PADD I) & 3,150 & 1,966 & $E_{2,777}$ & $E_{2,310}$ & $E_{1,799}$ & $E_{1,462}$ & $E_{1,621}$ & $E_{1,760}$ \\
Midwest (PADD II) & 10,699 & 7,653 & $E_{15,414}$ & $E_{13,367}$ & $E_{11,628}$ & $E_{10,555}$ & $E_{9,933}$ & $E_{9,146}$ \\
Gulf Coast (PADD III) & 18,757 & 15,855 & $E_{23,203}$ & $E_{21,163}$ & $E_{19,521}$ & $E_{17,284}$ & $E_{15,169}$ & $E_{13,225}$ \\
Total (PADD I-III) & 32,606 & 25,474 & $E_{41,394}$ & $E_{36,840}$ & $E_{32,948}$ & $E_{29,301}$ & $E_{26,723}$ & $E_{24,131}$ \\
U.S. Total & 33,489 & 26,174 & $E_{42,455}$ & $E_{37,785}$ & $E_{33,793}$ & $E_{30,052}$ & $E_{27,408}$ & $E_{24,750}$ \\
\hline
\end{tabular}

E=Estlmated data.

Source: Energy Information Administration (EIA), Monthly Petroleum Supply Reporting System and Form ElA-807,"Propane Telephone Survey."

\section{NATURAL GAS}

\section{Supply and Disposition}

The Energy Information Administration (EIA) estimates that total gas supply available for disposition in December 1993 was an estimated 2,207 billion cubic feet, 1 percent greater than in December 1992. The December 1993 total includes 13 billion cubic feet of supplemental fuel supplies, 198 billion cubic feet of imported gas, and 499 billion cubic feet withdrawn from storage.

O11 i: - disposition side, in December 1993, the consumption of 2,138 billion cubic feet was 24 percent greater than in December 1992. Total disposition included 58 billion cubic feet of gas injected into underground storage reservoirs and exports of 11 billion cubic feet.

\section{Consumption}

Data for the four major end-use sectors indicate that the total amount of gas delivered to all consumers increased to 1,572 billion cubic feet in November 1993, from 1,523 billion cubic feet in November 1992. Consumption in the industrial sector decreased from 653 billion cubic feet in October 1993 to 644 billion cubic feet in November 1993, a decrease of 1 percent.

The electric utility sector consumed 208 billion cubic feet in November 1993, which is 11 percent less than in October 1993 and a 10-percent increase from November 1992.

The residential sector consumed 455 billion cubic feet and the commercial sector consumed 264 billion cubic feet in November 1993.

\section{Natural Gas Prices}

In November 1993, major interstate pipeline companies paid an average of $\$ 2.32$ per thousand cubic feet for gas purchased from domestic producers, 13 percent greater than the October's $\$ 2.05$ total. In November 1993, these pipeline companies paid $\$ 1.85$ per thousand cubic feet for imported gas. Distributors paid an average of $\$ 3.14$ per thousand cubic feet for gas at the city gate in November 1993. Residential consumers paid $\$ 6.16$ per thousand cubic feet in November 1993, 2 percent higher than what they paid in November 1992. 


\section{PRICES}

Surprisingly, heating oil prices did not reflect the continued cold weather and declining stocks. Average wholesale prices fell 0.4 cent, from 61.6 to $61 . z$ cents per gallon. Average retail prices rose 0.8 cent, from 98.7 to 99.5 cents per gallon. Retail prices in all but the Midwest region, are presently above those of one year ago. The average retail price in the Midwest is 1.6 cents per gallon below that of one year ago. Uncertainty concerning future weather related demand contributed to conservative trading.

Average wholesale propane prices fell 1.0 cent, from 36.5 to 35.5 cents per gallon. Average retail prices rose 0.4 cent, from 90.5 to 90.9 cents per gallon. Once again the Central Atlantic region reported the largest price increase, 0.7 cent, from 122.7 to 123.4 cents per gallon. Despite cold weather and stock drawdowns, average retail prices are 2.7 cents below those of one year ago.

As a point of interest, both residential heating oil and propane prices for the month of January, were lower than average prices for January 1993. Average propane prices are 9.1 cents below January, 1993.

Table H3. Residentlal Heating Oll Prices by Petroleum Administration for Defense Districts (Cents per Gallon)

\begin{tabular}{lccccccccc}
\hline & December & January & \multicolumn{7}{c}{ Week Ending } \\
\cline { 7 - 10 } PAD Districts & $\mathbf{1 9 9 2}$ & $\mathbf{1 9 9 3}$ & $\mathbf{1 2 / 2 0 / 9 3}$ & $\mathbf{0 1 / 0 3 / 9 4}$ & $\mathbf{0 1 / 1 7 / 9 4}$ & $\mathbf{0 1 / 3 1 / 9 4}$ & $\mathbf{0 2 / 0 7 / 9 4}$ & $\mathbf{0 2 / 1 4 / 9 4}$ \\
\hline Average & 97.5 & 97.6 & 92.6 & 92.1 & 94.7 & 96.6 & 98.7 & 99.5 \\
East Coast & 99.3 & 99.5 & 94.3 & 94.1 & 96.7 & 98.8 & 101.0 & 101.9 \\
New England & 96.3 & 96.6 & 89.2 & 89.1 & 92.9 & 95.0 & 97.1 & 98.4 \\
Central Atlantic & 101.5 & 101.7 & 97.5 & 97.4 & 99.3 & 101.4 & 103.8 & 104.5 \\
Lower Atlantic & 93.4 & 93.3 & 87.5 & 87.5 & 89.8 & 92.7 & 93.7 & 93.6 \\
Midwest & 87.1 & 87.0 & 83.0 & 80.9 & 83.5 & 84.0 & 85.6 & 85.9 \\
\end{tabular}

Source: Based on data collected by State Energy Otfices.

Table H4. Residential Propane Prices by Petroleum Administration for Defense Districts (Cents per Gallon)

\begin{tabular}{|c|c|c|c|c|c|c|c|c|}
\hline \multirow[b]{2}{*}{ PAD Districts } & \multirow{2}{*}{$\begin{array}{c}\text { December } \\
1992\end{array}$} & \multirow{2}{*}{$\begin{array}{c}\text { January } \\
1993\end{array}$} & \multicolumn{6}{|c|}{ Week Ending } \\
\hline & & & $12 / 20 / 93$ & $01 / 03 / 94$ & $01 / 17 / 94$ & $01 / 31 / 94$ & $02 / 07 / 94$ & $02 / 14 / 94$ \\
\hline Average & 97.9 & 94.6 & 88.0 & 88.0 & 88.4 & 89.6 & 90.5 & 90.9 \\
\hline East Coast & 116.7 & 116.9 & 111.0 & 111.1 & 111.8 & 113.5 & 114.6 & 115.0 \\
\hline New England & 117.4 & 118.3 & 116.1 & 116.1 & 116.1 & 116.8 & 117.7 & 117.8 \\
\hline Central Atlantic & 127.3 & 127.0 & 118.7 & 118.7 & 119.9 & 121.0 & 122.7 & 123.4 \\
\hline Lower Atlantic & 102.2 & 102.0 & 96.8 & 96.9 & 97.9 & 100.9 & 101.5 & 101.7 \\
\hline Midwest & 87.7 & 82.3 & 74.8 & 74.7 & 75.1 & 75.9 & 76.7 & 76.8 \\
\hline
\end{tabular}

Source: Based on data collected by State Energy Offices. 


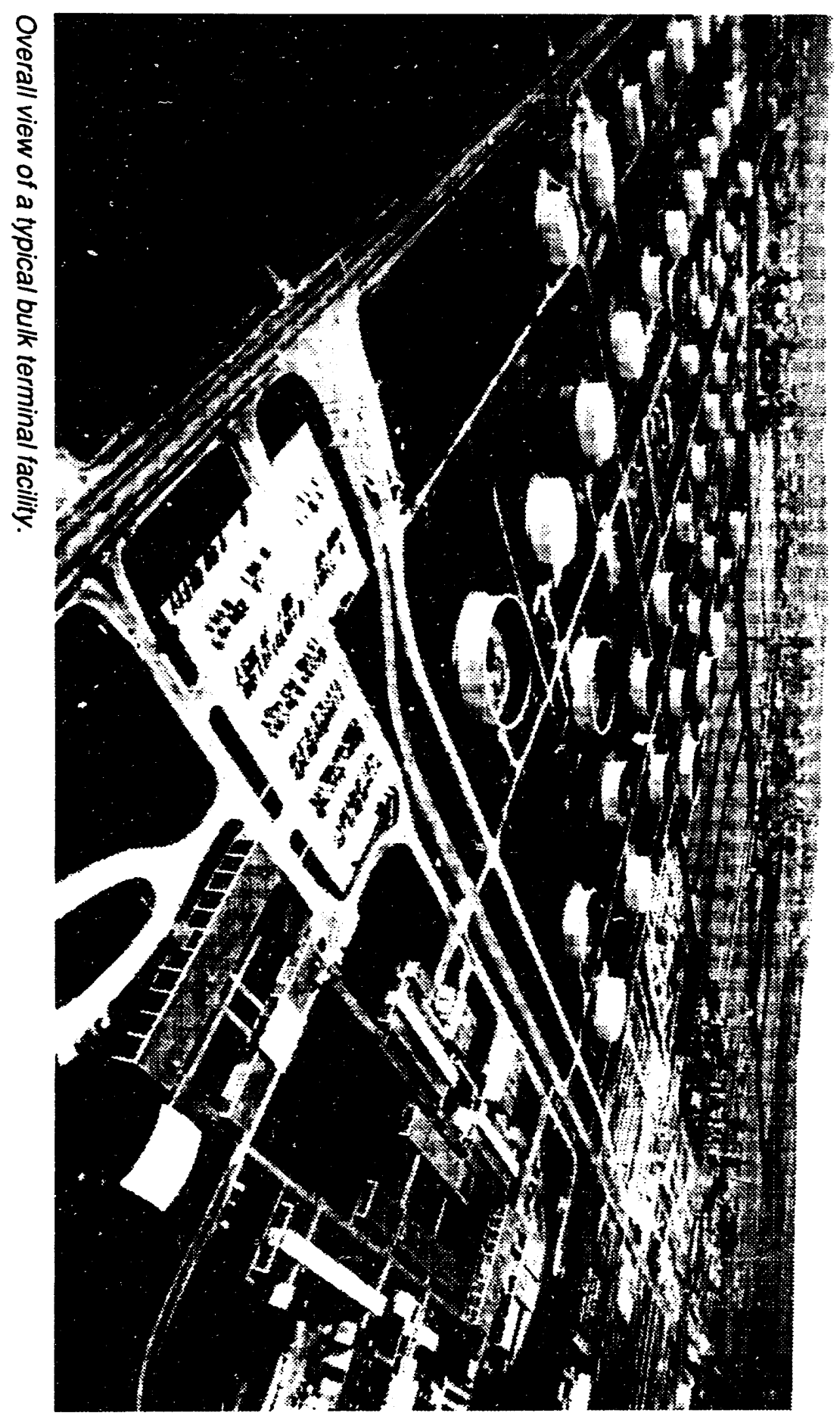

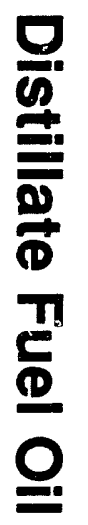


Table 1. Monthly and Weekly Net Production, Imports, and Stocks of Distlllate Fuel OII by Petroleum Administration for Defense District (PADD) and Product Supplied for the United States

(Thousand Barrels per Day, Except Where Noted)

\begin{tabular}{|c|c|c|c|c|c|c|c|c|c|c|c|c|}
\hline DistriceYear & Jan & Feb & Mar & Apr & May & Jun & Jul & Aug & Sep & Oct & Nov & Dec \\
\hline $\begin{array}{l}\text { Total U.S. } \\
\text { Net Production } \\
1991 \\
1992 \\
1993\end{array}$ & $\begin{array}{l}2,845 \\
2,818 \\
2,909\end{array}$ & $\begin{array}{l}2,870 \\
2,661 \\
2,813\end{array}$ & $\begin{array}{l}2,865 \\
2,749 \\
2,918\end{array}$ & $\begin{array}{l}2,819 \\
2,930 \\
3,010\end{array}$ & $\begin{array}{l}2,929 \\
2,933 \\
2,930\end{array}$ & $\begin{array}{l}2,941 \\
2,995 \\
3,095\end{array}$ & $\begin{array}{l}2,998 \\
3,067 \\
3,185\end{array}$ & $\begin{array}{l}2,961 \\
2,865 \\
3,084\end{array}$ & $\begin{array}{l}3,055 \\
2,983 \\
3,206\end{array}$ & $\begin{array}{l}3,040 \\
3,251 \\
3,435\end{array}$ & $\begin{array}{l}3,103 \\
3,240 \\
3,478\end{array}$ & $\begin{array}{l}3,107 \\
3,179\end{array}$ \\
\hline $\begin{array}{l}\text { Week Endling } \\
1993 / 1994 \\
\text { Tord/\% } \\
\text { 0.05\% Sulf \& Under } \\
\text { Greater than 0.05\% }\end{array}$ & $\begin{array}{l}12 / 03 \\
0,560 \\
2,042 \\
1,518\end{array}$ & $\begin{array}{l}12 / 10 \\
3,481 \\
1,752 \\
1,729\end{array}$ & $\begin{array}{c}12 / 17 \\
6,17 \% \\
1,732 \\
1,642\end{array}$ & $\begin{array}{l}12 / 24 \\
3,068 \\
1,643 \\
1,725\end{array}$ & $\begin{array}{r}12 / 3 i \\
6,510 \\
1,910 \\
1,600\end{array}$ & $\begin{array}{l}01 / 07 \\
0,364 \\
1,640 \\
1,724\end{array}$ & $\begin{array}{l}01 / 14 \\
3,52 \\
1,684 \\
1,768\end{array}$ & $\begin{array}{l}01 / 21 \\
0,088 \\
1,458 \\
1,630\end{array}$ & $\begin{array}{l}01 / 28 \\
3,101 \\
1,435 \\
1,666\end{array}$ & $\begin{array}{r}02 / 04 \\
0,6,42 \\
1,387 \\
1,755\end{array}$ & $\begin{array}{l}02 / 11 \\
3,257 \\
1,529 \\
1,728\end{array}$ & $\begin{array}{l}02 / 18 \\
3,189 \\
1,532 \\
1,657\end{array}$ \\
\hline $\begin{array}{l}\text { Imports } \\
1991 \\
1992 \\
1993\end{array}$ & $\begin{array}{l}192 \\
232 \\
182\end{array}$ & $\begin{array}{l}139 \\
217 \\
224\end{array}$ & $\begin{array}{l}206 \\
238 \\
235\end{array}$ & $\begin{array}{l}258 \\
202 \\
209\end{array}$ & $\begin{array}{l}186 \\
179 \\
153\end{array}$ & $\begin{array}{l}209 \\
157 \\
168\end{array}$ & $\begin{array}{l}155 \\
172 \\
130\end{array}$ & $\begin{array}{l}168 \\
229 \\
159\end{array}$ & $\begin{array}{l}237 \\
237 \\
137\end{array}$ & $\begin{array}{l}207 \\
263 \\
242\end{array}$ & $\begin{array}{l}249 \\
236 \\
214\end{array}$ & $\begin{array}{l}252 \\
229\end{array}$ \\
\hline $\begin{array}{l}\text { Week Endlng } \\
1993 / 1994 \\
\text { OTotal } \\
0.05 \% \text { Sulf \& Under } \\
\text { Greater than } 0.05 \%\end{array}$ & $\begin{array}{c}12 / 03 \\
2 / 8 \\
127 \\
86\end{array}$ & $\begin{array}{c}12 / 10 \\
1 / 13 \\
158\end{array}$ & $\begin{array}{c}12 / 17 \\
610 \\
24 \\
86\end{array}$ & $\begin{array}{l}12 / 24 \\
107 \\
39 \\
68\end{array}$ & $\begin{array}{c}12 / 31 \\
867 \\
64 \\
23\end{array}$ & $\begin{array}{r}01 / 07 \\
152 \\
40 \\
92\end{array}$ & $\begin{array}{c}01 / 14 \\
153 \\
18 \\
135\end{array}$ & $\begin{array}{c}01 / 21 \\
77 \\
61\end{array}$ & $\begin{array}{c}01 / 28 \\
286 \\
48 \\
178\end{array}$ & $\begin{array}{r}02 / 04 \\
226 \\
63 \\
160\end{array}$ & $\begin{array}{r}02 / 11 \\
359 \\
30 \\
329\end{array}$ & $\begin{array}{r}02 / 18 \\
349 \\
68 \\
281\end{array}$ \\
\hline $\begin{array}{l}\text { Stocks (Million Barr } \\
1991 \\
1992 \\
1993\end{array}$ & $\begin{array}{l}111.7 \\
126.7 \\
130.2\end{array}$ & $\begin{array}{l}101.6 \\
108.8 \\
109.4\end{array}$ & $\begin{array}{l}98.2 \\
97.7 \\
97.5\end{array}$ & $\begin{array}{r}102.9 \\
92.1 \\
98.3\end{array}$ & $\begin{array}{r}106.9 \\
96.4 \\
101.6\end{array}$ & $\begin{array}{l}113.7 \\
104.5 \\
109.4\end{array}$ & $\begin{array}{l}124.7 \\
114.6 \\
120.2\end{array}$ & $\begin{array}{l}131.4 \\
122.8 \\
127.9\end{array}$ & $\begin{array}{l}140.1 \\
127.8 \\
130.4\end{array}$ & $\begin{array}{l}138.3 \\
136.8 \\
144.8\end{array}$ & $\begin{array}{l}144.5 \\
146.3 \\
149.5\end{array}$ & $\begin{array}{l}143.5 \\
140.6\end{array}$ \\
\hline $\begin{array}{l}\text { Weok Ending } \\
1993 / 1994 \\
\text { Tota/ } \\
0.05 \% \text { Sulf \& Under } \\
\text { Greater than } 0.05 \%\end{array}$ & $\begin{array}{r}12 / 03 \\
646.2 \\
61.5 \\
84.7\end{array}$ & $\begin{array}{r}12 / 10 \\
14.17 \\
61.8 \\
82.8\end{array}$ & $\begin{array}{r}12 / 17 \\
140.8 \\
61.8 \\
79.0\end{array}$ & $\begin{array}{r}12 / 24 \\
142.5 \\
61.4 \\
81.1\end{array}$ & $\begin{array}{c}12 / 31 \\
1447 \\
62.8 \\
81.9\end{array}$ & $\begin{array}{r}01 / 07 \\
140.0 \\
63.3 \\
76.7\end{array}$ & $\begin{array}{c}01 / 14 \\
134.6 \\
61.4 \\
73.2\end{array}$ & $\begin{array}{r}01 / 21 \\
125, \\
58.5 \\
67.2\end{array}$ & $\begin{array}{r}01 / 28 \\
1167 \\
52.9 \\
63.9\end{array}$ & $\begin{array}{c}02 / 04 \\
108.3 \\
50.9 \\
57.3\end{array}$ & $\begin{array}{c}02 / 11 \\
1047 \\
48.8 \\
55.8\end{array}$ & $\begin{array}{r}02 / 18 \\
102.5 \\
47.4 \\
55.1\end{array}$ \\
\hline $\begin{array}{l}\text { Product Supplled } \\
1991 \\
1992 \\
1993\end{array}$ & $\begin{array}{l}3,367 \\
3,231 \\
3,141\end{array}$ & $\begin{array}{l}2,976 \\
3,219 \\
3,478\end{array}$ & $\begin{array}{l}2,984 \\
3,207 \\
3,386\end{array}$ & $\begin{array}{l}2,839 \\
3,039 \\
2,949\end{array}$ & $\begin{array}{l}2,765 \\
2,753 \\
2,624\end{array}$ & $\begin{array}{l}2,775 \\
2,679 \\
2,843\end{array}$ & $\begin{array}{l}2,648 \\
2,710 \\
2,669\end{array}$ & $\begin{array}{l}2,770 \\
2,705 \\
2,797\end{array}$ & $\begin{array}{l}2,865 \\
2,908 \\
3,001\end{array}$ & $\begin{array}{l}3,047 \\
3,056 \\
2,968\end{array}$ & $\begin{array}{l}2,921 \\
2,929 \\
3,206\end{array}$ & $\begin{array}{l}3,087 \\
3,316\end{array}$ \\
\hline $\begin{array}{l}\text { Weak Endling } \\
1993 / 1994 \\
\text { '/, }\end{array}$ & $\begin{array}{r}12 / 03 \\
2,965\end{array}$ & $\begin{array}{r}12 / 10 \\
3,700\end{array}$ & $\begin{array}{r}12 / 17 \\
3,865\end{array}$ & $\begin{array}{r}12 / 24 \\
3,057\end{array}$ & $\begin{array}{l}12 / 31 \\
3 / 103\end{array}$ & $\begin{array}{l}01 / 07 \\
4,002\end{array}$ & $\begin{array}{r}01 / 14 \\
4,212\end{array}$ & $\begin{array}{l}01 / 21 \\
4,277\end{array}$ & $\begin{array}{r}01 / 28 \\
4.436\end{array}$ & $\begin{array}{l}02 / 04 \\
4410\end{array}$ & $\begin{array}{c}02 / 11 \\
3,965\end{array}$ & $\begin{array}{l}02 / 18 \\
3,685\end{array}$ \\
\hline $\begin{array}{l}\text { East Coast (PADD I) } \\
\text { Net Production }{ }^{\mathrm{a}} \\
1991 \\
1992 \\
1993\end{array}$ & $\begin{array}{l}344 \\
332 \\
370\end{array}$ & $\begin{array}{l}373 \\
292 \\
335\end{array}$ & $\begin{array}{l}344 \\
275 \\
335\end{array}$ & $\begin{array}{l}299 \\
371 \\
359\end{array}$ & $\begin{array}{l}339 \\
355 \\
322\end{array}$ & $\begin{array}{l}367 \\
369 \\
426\end{array}$ & $\begin{array}{l}368 \\
406 \\
417\end{array}$ & $\begin{array}{l}359 \\
352 \\
375\end{array}$ & $\begin{array}{l}376 \\
361 \\
393\end{array}$ & $\begin{array}{l}351 \\
448 \\
468\end{array}$ & $\begin{array}{l}383 \\
426 \\
456\end{array}$ & $\begin{array}{l}395 \\
395\end{array}$ \\
\hline $\begin{array}{l}\text { Week Ending } \\
1993,1994 \\
\text { Total } \\
\text { 0.05\% Sulf \& Under } \\
\text { Greater than } 0.05 \%\end{array}$ & $\begin{array}{r}12 / 03 \\
509 \\
264 \\
245\end{array}$ & $\begin{array}{c}12 / 10 \\
505 \\
135 \\
370\end{array}$ & $\begin{array}{c}12 / 17 \\
482 \\
184 \\
298\end{array}$ & $\begin{array}{c}12 / 24 \\
467 \\
85 \\
372\end{array}$ & $\begin{array}{l}12 / 34 \\
470 \\
203 \\
267\end{array}$ & $\begin{array}{c}01 / 07 \\
457 \\
138 \\
319\end{array}$ & $\begin{array}{c}01 / 14 \\
461 \\
83 \\
378\end{array}$ & $\begin{array}{l}01 / 21 \\
370 \\
104 \\
266\end{array}$ & $\begin{array}{c}01 / 28 \\
418 \\
92 \\
326\end{array}$ & $\begin{array}{l}02 / 04 \\
459 \\
116 \\
343\end{array}$ & $\begin{array}{r}02 / 11 \\
533 \\
121 \\
412\end{array}$ & $\begin{array}{c}02 / 18 \\
455 \\
120 \\
335\end{array}$ \\
\hline $\begin{array}{l}\text { Stocks (Million Bar } \\
1991 \\
1992 \\
1993\end{array}$ & is) $\begin{array}{l} \\
39.8 \\
53.4 \\
58.6\end{array}$ & $\begin{array}{l}31.8 \\
43.5 \\
43.2\end{array}$ & $\begin{array}{l}29.8 \\
31.0 \\
33.1\end{array}$ & $\begin{array}{l}32.3 \\
28.5 \\
34.5\end{array}$ & $\begin{array}{l}35.5 \\
30.1 \\
37.1\end{array}$ & $\begin{array}{l}43.6 \\
37.5 \\
43.2\end{array}$ & $\begin{array}{l}51.0 \\
45.4 \\
51.5\end{array}$ & $\begin{array}{l}56.6 \\
53.6 \\
59.2\end{array}$ & $\begin{array}{l}62.3 \\
58.1 \\
63.8\end{array}$ & $\begin{array}{l}65.6 \\
64.8 \\
72.0\end{array}$ & $\begin{array}{l}66.8 \\
68.2 \\
70.1\end{array}$ & $\begin{array}{l}63.4 \\
65.1\end{array}$ \\
\hline $\begin{array}{l}\text { Week Ending } \\
1993 / 1994 \\
\text { Total } \\
\text { O.05\% Sulf \& Under } \\
\text { Greater than 0.05\% }\end{array}$ & $\begin{array}{c}12 / 03 \\
698 \\
19.0 \\
50.8\end{array}$ & $\begin{array}{c}12 / 10 \\
6609 \\
18.1 \\
48.7\end{array}$ & $\begin{array}{c}12 / 17 \\
64.9 \\
18.7 \\
46.3\end{array}$ & $\begin{array}{c}12 / 24 \\
65.9 \\
18.9 \\
47.0\end{array}$ & $\begin{array}{c}12 / 31 \\
66,3 \\
19.4 \\
47.0\end{array}$ & $\begin{array}{c}01 / 07 \\
59.5 \\
18.4 \\
41.1\end{array}$ & $\begin{array}{c}01 / 14 \\
55.8 \\
16.5 \\
39.3\end{array}$ & $\begin{array}{r}01 / 21 \\
479 \\
15.4 \\
32.4\end{array}$ & $\begin{array}{r}01 / 28 \\
43.2 \\
13.8 \\
29.4\end{array}$ & $\begin{array}{c}02 / 04 \\
35.8 \\
11.8 \\
24.0\end{array}$ & $\begin{array}{r}02 / 11 \\
34.8 \\
11.6 \\
23.2\end{array}$ & $\begin{array}{r}02 / 18 \\
33.6 \\
10.2 \\
23.3\end{array}$ \\
\hline
\end{tabular}

See footnotes at end of table. 
Table 1. Monthly and Weekly Net Production, Imports, and Stocks of Distillate Fuel OII by Petroleum Administration for Defense District (PADD) and Product Supplled for the United States (Continued) (Thousand Barrels per Day, Except Where Noted)

\begin{tabular}{|c|c|c|c|c|c|c|c|c|c|c|c|c|}
\hline DistrictYear & Jan & $F \bullet b$ & Mar & Apr & May & Jun & Jul & Aug & Sep & Oct & Nov & Dec \\
\hline $\begin{array}{l}\text { New England (PADD } \\
\text { Stocks (Million Ba } \\
1991 \\
1992 \\
1993\end{array}$ & $\begin{array}{r}5.4 \\
7.4 \\
10.0\end{array}$ & $\begin{array}{l}3.6 \\
6.7 \\
8.0\end{array}$ & $\begin{array}{l}3.5 \\
4.4 \\
5.8\end{array}$ & $\begin{array}{l}4.4 \\
3.3 \\
5.3\end{array}$ & $\begin{array}{l}5.1 \\
4.7 \\
5.5\end{array}$ & $\begin{array}{l}6.5 \\
6.8 \\
7.7\end{array}$ & $\begin{array}{l}8.7 \\
9.5 \\
8.9\end{array}$ & $\begin{array}{r}9.9 \\
11.0 \\
10.5\end{array}$ & $\begin{array}{l}10.8 \\
11.2 \\
10.5\end{array}$ & $\begin{array}{l}11.0 \\
12.1 \\
13.0\end{array}$ & $\begin{array}{l}11.8 \\
11.6 \\
11.9\end{array}$ & $\begin{array}{l}9.9 \\
9.9\end{array}$ \\
\hline $\begin{array}{l}\text { Week Ending } \\
1993 / 1994\end{array}$ & $12 / 03$ & $12 / 10$ & $12 / 17$ & $12 / 24$ & $12 / 31$ & $01 / 07$ & $01 / 14$ & $01 / 21$ & $01 / 28$ & $02 / 04$ & $02 / 11$ & $02 / 18$ \\
\hline $\begin{array}{l}\text { Toud } \\
0.05 \% \text { Sulf \& Under } \\
\text { Greater than } 0.05 \%\end{array}$ & $\begin{array}{r}13.3 \\
3.0 \\
10.2\end{array}$ & $\begin{array}{c}16.6 \\
3.0 \\
9.6\end{array}$ & $\begin{array}{c}12.0 \\
3.1 \\
8.9\end{array}$ & $\begin{array}{c}1 \% \\
2.9 \\
8.9\end{array}$ & $\begin{array}{c}12.0 \\
2.7 \\
9.3\end{array}$ & $\begin{array}{l}11.6 \\
2.6 \\
8.6\end{array}$ & $\begin{array}{c}10.6 \\
2.4 \\
7.9\end{array}$ & $\begin{array}{c}6.3 \\
2.4 \\
5.9\end{array}$ & $\begin{array}{l}7.3 \\
2.0 \\
5.4\end{array}$ & $\begin{array}{l}6.6 \\
1.8 \\
4.9\end{array}$ & $\begin{array}{l}6.9 \\
1.7 \\
5.2\end{array}$ & $\begin{array}{r}5.0 \\
1.2 \\
3.8\end{array}$ \\
\hline
\end{tabular}

Central Atlantic (PADD M

Stocks (Mililen Barrels)

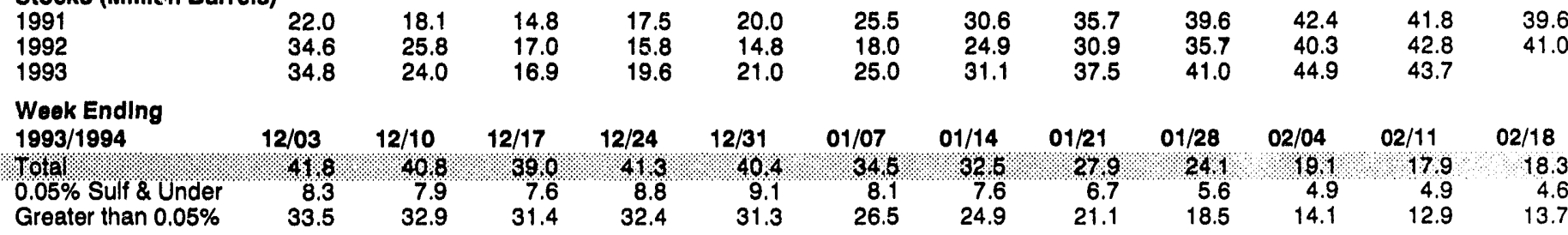

Lower Atlantic (PADD IZ)

Stocks (Million Barrels)

\begin{tabular}{|c|c|c|c|c|c|c|c|c|c|c|c|c|}
\hline $\begin{array}{l}1991 \\
1992 \\
1993\end{array}$ & $\begin{array}{l}12.4 \\
11.3 \\
13.8\end{array}$ & $\begin{array}{l}10.0 \\
11.0 \\
11.1\end{array}$ & $\begin{array}{r}11.4 \\
9.5 \\
10.5\end{array}$ & $\begin{array}{r}10.4 \\
9.4 \\
9.6\end{array}$ & $\begin{array}{l}10.3 \\
10.6 \\
10.6\end{array}$ & $\begin{array}{l}11.6 \\
12.7 \\
10.5\end{array}$ & $\begin{array}{l}11.6 \\
11.1 \\
11.6\end{array}$ & $\begin{array}{l}11.0 \\
11.7 \\
11.2\end{array}$ & $\begin{array}{l}11.9 \\
11.3 \\
12.3\end{array}$ & $\begin{array}{l}12.2 \\
12.4 \\
14.1\end{array}$ & $\begin{array}{l}13.3 \\
13.7 \\
14.5\end{array}$ & $\begin{array}{l}13.9 \\
14.1\end{array}$ \\
\hline $\begin{array}{l}\text { Woek Endlng } \\
1993 / 1994\end{array}$ & $12 / 03$ & $12 / 10$ & $12 / 17$ & $12 / 24$ & $12 / 31$ & 01/07 & $01 / 14$ & $01 / 21$ & $01 / 28$ & $02 / 04$ & $02 / 11$ & $02 / 1$ \\
\hline $\begin{array}{l}\text { Total } \\
0.05 \% \text { Sulf \& Under } \\
\text { Greater than } 0.05 \%\end{array}$ & $\begin{array}{l}147 \\
7.6 \\
7.1\end{array}$ & $\begin{array}{c}10.6 \\
7.2 \\
6.3\end{array}$ & $\begin{array}{r}13.9 \\
8.0 \\
6.0\end{array}$ & $\begin{array}{r}12.8 \\
7.2 \\
5.7\end{array}$ & $\begin{array}{c}140 \\
7.6 \\
6.4\end{array}$ & $\begin{array}{c}13.8 \\
7.7 \\
6.1\end{array}$ & $\begin{array}{l}10.0 \\
6.5 \\
6.5\end{array}$ & $\begin{array}{r}11.7 \\
6.4 \\
5.4\end{array}$ & $\begin{array}{r}11.7 \\
6.2 \\
5.6\end{array}$ & $\begin{array}{r}10.1 \\
5.1 \\
5.0\end{array}$ & $\begin{array}{r}10.1 \\
5.0 \\
5.1\end{array}$ & \\
\hline
\end{tabular}

Midwest (PADD II)

Net Production

199

1992

1993

$\begin{array}{lll}665 & 679 & 677 \\ 683 & 685 & 700 \\ 757 & 692 & 72\end{array}$

$\begin{array}{llll}677 & 679 & 724 & 734\end{array}$

700
724

$\begin{array}{ll}654 & 722 \\ 747 & 733\end{array}$

$12 / 03$

1993/1994

Total

$0.05 \%$ Sulf \& Under

890

503

387

$12 / 10$

$12 / 10 \quad 12 / 17$

434
418

849
423
426

$12 / 2$

$12 / 24 \quad 12 / 31$

426
438

$12 / 31$
869
393

Stocks (MIllion Barrels)

1991

1992

1993

31.

31.2
32.1

29.8

29.8

$\begin{array}{lll}30.0 & 30.6 & 31.6\end{array}$

$\begin{array}{lll}30.1 & 27.7 & 27.4\end{array}$

29.0

28.3

Week Ending

1993/1994

Total:

$0.05 \%$ Sulf \& Under

$12 / 03$

$12 / 03$
30
20
12

$30:$
20
12.9

$12 / 10$

$12 / 17$

337 .

$12 / 24 \quad 12 / 31$

33.6 36.

$20.8 \quad 23.1$

$13.0 \quad 13.0$ $\begin{array}{lll}13.4 & 12.9 & 13.0\end{array}$
26.9

\begin{abstract}
13.0
\end{abstract}

734
739
753

$01 / 07$
797
358
439

$\begin{array}{ll}769 & 71 \\ 739 & 743 \\ 756 & 700\end{array}$

711
743
700

74
738
756

778

774
86

$01 / 14 \quad 01 / 21$

$422 \quad 373$

373
390

$01 / 28$
720
328

392

$31.2 \quad 33.1$

29.0

27.7

29.3

$01 / 07$

36.5
23.5
12.9
$01 / 14$
34.8

12.8

33.2
31.1
27.3

$01 / 21$
33.9
21.3
12.6

32.1
30.8

27.1

30.4

30.4
29.1

$01 / 28$

31.6
19.8
11.7

$02 / 04$

30.6
19.2

11.3

$\begin{array}{cc}746 & 734 \\ 779 & 768 \\ 875 & \\ & \\ 02 / 11 & 02 / 18 \\ 772 & 771 \\ 363 & 370 \\ 409 & 401\end{array}$

401

33.0

$31.9 \quad 31.3$

33.9

$02 / 11 \quad 02 / 18$

$\begin{array}{ll}29.5 & 29.7\end{array}$

$18.2 \quad 17.6$

See footnotes at end of table. 
Table 1. Monthly and Weekly Net Production, Imports, and Stocks of Distlllate Fuel OII by Petroleum Administration for Defense District (PADD) and Product Supplled for the United States (Continued) (Thousand Barrels per Day, Except Where Noted)

\begin{tabular}{|c|c|c|c|c|c|c|c|c|c|c|c|c|}
\hline Dlstrlct/Year & Jan & Feb & Mar & Apr & May & Jun & Jul & Aug & Sep & Oct & Nov & Dec \\
\hline $\begin{array}{l}\text { Gulf Coast (PADD III) } \\
\text { Net Productlon } \\
1991 \\
1992 \\
1993\end{array}$ & $\begin{array}{l}1,286 \\
1,274 \\
1,300\end{array}$ & $\begin{array}{l}1,293 \\
1,170 \\
1,271\end{array}$ & $\begin{array}{l}1,328 \\
1,220 \\
1,315\end{array}$ & $\begin{array}{l}1,295 \\
1,327 \\
1,349\end{array}$ & $\begin{array}{l}1,292 \\
1,302 \\
1,281\end{array}$ & $\begin{array}{l}1,264 \\
1,314 \\
1,342\end{array}$ & $\begin{array}{l}1,297 \\
1,348 \\
1,430\end{array}$ & $\begin{array}{l}1,329 \\
1,205 \\
1,466\end{array}$ & $\begin{array}{l}1,344 \\
1,323 \\
1,444\end{array}$ & $\begin{array}{l}1,332 \\
1,452 \\
1,488\end{array}$ & $\begin{array}{l}1,410 \\
1,486 \\
1,524\end{array}$ & $\begin{array}{l}1,422 \\
1,462\end{array}$ \\
\hline $\begin{array}{l}\text { Weok Ending } \\
1993 / 1994 \\
\text { tota/ } \\
0.05 \% \text { Sulf \& Under } \\
\text { Greater than 0.05\% }\end{array}$ & $\begin{array}{c}12 / 03 \\
1595 \\
909 \\
686\end{array}$ & $\begin{array}{c}12 / 10 \\
1646 \\
803 \\
739\end{array}$ & $\begin{array}{c}12 / 17 \\
\% \neq 2 / \% \\
709 \\
713\end{array}$ & $\begin{array}{c}12 / 24 \\
1586 \\
799 \\
739\end{array}$ & $\begin{array}{c}12 / 31 \\
691 \% \\
841 \\
750\end{array}$ & $\begin{array}{c}01 / 07 \\
1 \% 80 \\
717 \\
772\end{array}$ & $\begin{array}{l}01 / 14 \\
1698 \\
816 \\
777\end{array}$ & $\begin{array}{l}01 / 21 \\
1391 \\
609 \\
782\end{array}$ & $\begin{array}{c}01 / 28 \\
1.25 \\
645 \\
780\end{array}$ & $\begin{array}{l}02 / 04 \\
1,417 \\
557 \\
860\end{array}$ & $\begin{array}{c}02 / 11 \\
1346 \\
641 \\
705\end{array}$ & $\begin{array}{r}02 / 18 \\
1,392 \\
669 \\
723\end{array}$ \\
\hline $\begin{array}{l}\text { Stocks (Million Barr } \\
1991 \\
1992 \\
1993\end{array}$ & $\begin{array}{l}27.2 \\
28.8 \\
27.1\end{array}$ & $\begin{array}{l}25.9 \\
22.5 \\
24.6\end{array}$ & $\begin{array}{l}25.1 \\
23.4 \\
23.1\end{array}$ & $\begin{array}{l}26.7 \\
24.0 \\
23.4\end{array}$ & $\begin{array}{l}25.5 \\
25.6 \\
24.1\end{array}$ & $\begin{array}{l}24.7 \\
24.7 \\
25.3\end{array}$ & $\begin{array}{l}27.4 \\
27.1 \\
26.7\end{array}$ & $\begin{array}{l}28.6 \\
26.4 \\
29.3\end{array}$ & $\begin{array}{l}31.0 \\
27.5 \\
28.4\end{array}$ & $\begin{array}{l}28.5 \\
31.5 \\
29.7\end{array}$ & $\begin{array}{l}31.2 \\
33.2 \\
30.8\end{array}$ & $\begin{array}{l}31.7 \\
30.8\end{array}$ \\
\hline $\begin{array}{l}\text { Weak Ending } \\
1993 / 1994 \\
\text { Tolal } \\
\text { 0.05\% Sulf \& Under } \\
\text { Greater than 0.05\% }\end{array}$ & $\begin{array}{r}12 / 03 \\
138.6 \\
13.6 \\
14.9\end{array}$ & $\begin{array}{l}12 / 10 \\
28.8 .8 \\
14.2 \\
14.6\end{array}$ & $\begin{array}{r}12 / 17 \\
27.4 \\
13.0 \\
14.4\end{array}$ & $\begin{array}{l}12 / 24 \\
28.6 \\
12.9 \\
15.7\end{array}$ & $\begin{array}{r}12 / 31 \\
28.2 \\
11.9 \\
16.3\end{array}$ & $\begin{array}{c}01 / 07 \\
29.4 \\
12.1 \\
17.3\end{array}$ & $\begin{array}{l}01 / 14 \\
30.1 \\
13.8 \\
16.2\end{array}$ & $\begin{array}{r}01 / 21 \\
29.8 \\
12.6 \\
17.3\end{array}$ & $\begin{array}{r}01 / 28 \\
28.4 \\
10.7 \\
17.7\end{array}$ & $\begin{array}{l}02 / 04 \\
29.1 \\
11.5 \\
17.6\end{array}$ & $\begin{array}{r}02 / 11 \\
27.5 \\
10.6 \\
16.9\end{array}$ & $\begin{array}{r}02 / 18 \\
26.1 \\
11.1 \\
15.0\end{array}$ \\
\hline $\begin{array}{l}\text { Rocky Mountain (PAD } \\
\text { Net Production } \\
1991 \\
1992 \\
1993\end{array}$ & $\begin{array}{l}\text { IV } \\
118 \\
112 \\
103\end{array}$ & $\begin{array}{l}113 \\
116 \\
109\end{array}$ & $\begin{array}{l}131 \\
126 \\
113\end{array}$ & $\begin{array}{l}122 \\
117 \\
109\end{array}$ & $\begin{array}{l}133 \\
119 \\
132\end{array}$ & $\begin{array}{l}136 \\
125 \\
125\end{array}$ & $\begin{array}{l}147 \\
128 \\
121\end{array}$ & $\begin{array}{l}139 \\
120 \\
124\end{array}$ & $\begin{array}{l}126 \\
122 \\
149\end{array}$ & $\begin{array}{l}136 \\
131 \\
134\end{array}$ & $\begin{array}{l}123 \\
120 \\
141\end{array}$ & $\begin{array}{l}118 \\
116\end{array}$ \\
\hline $\begin{array}{l}\text { Week Ending } \\
1993 / 1994 \\
\text { rotal } \\
0.05 \% \text { Sulf \& Under } \\
\text { Greater than 0.05\% }\end{array}$ & $\begin{array}{c}12 / 03 \\
125 \\
73 \\
52\end{array}$ & $\begin{array}{c}12 / 10 \\
112 \\
62 \\
50\end{array}$ & $\begin{array}{c}12 / 17 \\
109 \\
75 \\
64\end{array}$ & $\begin{array}{l}12 / 24 \\
119 \\
59 \\
60\end{array}$ & $\begin{array}{c}12 / 31 \\
132 \\
81 \\
51\end{array}$ & $\begin{array}{c}01 / 07 \\
129 \\
78 \\
51\end{array}$ & $\begin{array}{l}01 / 14 \\
1 / 24 \\
81 \\
43\end{array}$ & $\begin{array}{c}01 / 21 \\
129 \\
80 \\
49\end{array}$ & $\begin{array}{c}01 / 28 \\
133 \\
72 \\
61\end{array}$ & $\begin{array}{c}02 / 04 \\
116 \\
62 \\
54\end{array}$ & $\begin{array}{r}02 / 11 \\
129 \\
79 \\
50\end{array}$ & $\begin{array}{c}02 / 18 \\
125 \\
83 \\
42\end{array}$ \\
\hline $\begin{array}{l}\text { Stocks (Million Barr } \\
1991 \\
1992 \\
1993\end{array}$ & $\begin{array}{l}3.2 \\
2.7 \\
2.5\end{array}$ & $\begin{array}{l}3.3 \\
2.5 \\
2.4\end{array}$ & $\begin{array}{l}3.5 \\
2.8 \\
2.4\end{array}$ & $\begin{array}{l}3.1 \\
2.3 \\
2.0\end{array}$ & $\begin{array}{l}3.3 \\
2.2 \\
2.4\end{array}$ & $\begin{array}{l}3.3 \\
2.4 \\
2.3\end{array}$ & $\begin{array}{l}3.2 \\
2.5 \\
2.4\end{array}$ & $\begin{array}{l}3.0 \\
2.1 \\
2.1\end{array}$ & $\begin{array}{l}2.8 \\
2.0 \\
2.2\end{array}$ & $\begin{array}{l}2.6 \\
2.3 \\
2.1\end{array}$ & $\begin{array}{l}2.8 \\
2.7 \\
2.7\end{array}$ & $\begin{array}{l}3.2 \\
2.6\end{array}$ \\
\hline $\begin{array}{l}\text { Week Ending } \\
1993 / 1994 \\
\text { Total } \\
0.05 \% \text { Sulf \& Under } \\
\text { Greater than 0.05\% }\end{array}$ & $\begin{array}{r}12 / 03 \\
125 \\
1.5 \\
1.0\end{array}$ & $\begin{array}{c}12 / 10 \\
28 \\
1.7 \\
1.1\end{array}$ & $\begin{array}{r}12 / 17 \\
2.8 \\
1.7 \\
1.1\end{array}$ & $\begin{array}{c}12 / 24 \\
2.8 \\
1.6 \\
1.1\end{array}$ & $\begin{array}{r}12 / 31 \\
2.9 \\
1.7 \\
1.2\end{array}$ & $\begin{array}{r}01 / 07 \\
1.9 \\
1.6 \\
1.2\end{array}$ & $\begin{array}{c}01 / 14 \\
2 / 8 \\
1.7 \\
1.1\end{array}$ & $\begin{array}{r}01 / 21 \\
0.0 \\
1.8 \\
1.2\end{array}$ & $\begin{array}{c}01 / 28 \\
2.9 \\
1.6 \\
1.2\end{array}$ & $\begin{array}{r}02 / 04 \\
2.8 \\
1.7 \\
1.1\end{array}$ & $\begin{array}{r}02 / 11 \\
2.8 \\
1.7 \\
1.1\end{array}$ & $\begin{array}{r}02 / 18 \\
2.8 \\
1.7 \\
1.2\end{array}$ \\
\hline $\begin{array}{l}\text { West Coast (PADD V) } \\
\text { Net Production } \\
1991 \\
1992 \\
1993\end{array}$ & $\begin{array}{l}432 \\
418 \\
379\end{array}$ & $\begin{array}{l}411 \\
398 \\
406\end{array}$ & $\begin{array}{l}385 \\
427 \\
432\end{array}$ & $\begin{array}{l}424 \\
462 \\
446\end{array}$ & $\begin{array}{l}441 \\
436 \\
462\end{array}$ & $\begin{array}{l}440 \\
448 \\
450\end{array}$ & $\begin{array}{l}418 \\
446 \\
461\end{array}$ & $\begin{array}{l}423 \\
446 \\
419\end{array}$ & $\begin{array}{l}467 \\
441 \\
465\end{array}$ & $\begin{array}{l}442 \\
447 \\
482\end{array}$ & $\begin{array}{l}442 \\
428 \\
481\end{array}$ & $\begin{array}{l}438 \\
438\end{array}$ \\
\hline $\begin{array}{l}\text { Week Ending } \\
1993 / 1994 \\
\text { Total } \\
\text { O.05\% Sulf \& Under } \\
\text { Greater than } 0.05 \%\end{array}$ & $\begin{array}{l}12 / 03 \\
144 \\
293 \\
148\end{array}$ & $\begin{array}{l}12 / 10 \\
470 \\
318 \\
152\end{array}$ & $\begin{array}{l}12 / 17 \\
341 \\
141\end{array}$ & $\begin{array}{l}12 / 24 \\
390 \\
274 \\
116\end{array}$ & $\begin{array}{l}12 / 31 \\
440 \\
309 \\
139\end{array}$ & $\begin{array}{l}01 / 07 \\
492 \\
349 \\
143\end{array}$ & $\begin{array}{l}01 / 14 \\
420 \\
282 \\
138\end{array}$ & $\begin{array}{l}01 / 21 \\
435 \\
292 \\
143\end{array}$ & $\begin{array}{l}01 / 28 \\
405 \\
298 \\
107\end{array}$ & $\begin{array}{l}02 / 04 \\
393 \\
290 \\
103\end{array}$ & $\begin{array}{l}02 / 11 \\
477 \\
325 \\
152\end{array}$ & $\begin{array}{c}02 / 18 \\
446 \\
290 \\
156\end{array}$ \\
\hline $\begin{array}{l}\text { Stocks (Million Barr } \\
1991 \\
1992 \\
1993\end{array}$ & $\begin{array}{r} \\
11.5 \\
10.7 \\
9.9\end{array}$ & $\begin{array}{l}10.9 \\
10.4 \\
10.1\end{array}$ & $\begin{array}{r}9.9 \\
10.4 \\
9.9\end{array}$ & $\begin{array}{r}10.2 \\
9.6 \\
10.2\end{array}$ & $\begin{array}{l}11.1 \\
11.1 \\
11.0\end{array}$ & $\begin{array}{l}10.9 \\
10.8 \\
10.9\end{array}$ & $\begin{array}{l}10.0 \\
10.4 \\
10.9\end{array}$ & $\begin{array}{r}10.0 \\
9.6 \\
10.0\end{array}$ & $\begin{array}{r}11.9 \\
9.5 \\
8.9\end{array}$ & $\begin{array}{r}11.3 \\
9.1 \\
10.5\end{array}$ & $\begin{array}{l}11.5 \\
10.3 \\
12.0\end{array}$ & $\begin{array}{l}12.1 \\
10.8\end{array}$ \\
\hline $\begin{array}{l}\text { Week Ending } \\
1993 / 1994 \\
\text { Totd } \\
\text { O.05\% Sulf \& Under } \\
\text { Greater than } 0.05 \%\end{array}$ & $\begin{array}{c}12 / 03 \\
1 / 20 \\
6.9 \\
5.1\end{array}$ & $\begin{array}{c}12 / 10 \\
1 / 7 \\
6.7 \\
5.0\end{array}$ & $\begin{array}{c}12 / 17 \\
1 / 0 \\
7.6 \\
4.3\end{array}$ & $\begin{array}{l}12 / 24 \\
11.4 \\
7.1 \\
4.3\end{array}$ & $\begin{array}{c}12 / 31 \\
6.8 \\
6.8\end{array}$ & $\begin{array}{r}01 / 07 \\
1 / 8 \\
7.6 \\
4.1\end{array}$ & $\begin{array}{c}01 / 14 \\
11.2 \\
7.4 \\
3.7\end{array}$ & $\begin{array}{c}01 / 21 \\
1 / 10 \\
7.3 \\
3.7\end{array}$ & $\begin{array}{c}01 / 28 \\
10.6 \\
6.9 \\
3.7\end{array}$ & $\begin{array}{c}02 / 04 \\
10.0 \\
6.7 \\
3.2\end{array}$ & $\begin{array}{c}02 / 11 \\
10.0 \\
6.8 \\
3.2\end{array}$ & $\begin{array}{r}02 / 18 \\
10.3 \\
6.8 \\
3.5\end{array}$ \\
\hline
\end{tabular}

- Net production equals gross production minus input. Negative production will occur when the amount of product produced during the month is less than the amount of that same product reprocessed (input) or reclassified to become another product during the same month.

Notes: - Totals may not equal sum of components due to independent rounding. - Sum of PADD's IX, IY, and IZ may not equal PADD I because of independent estimation.

Source: Energy Information Administration, Weekly and Monthly Petroleum Supply Reporting Systems. Magnitudes of revisions to monthly data are published in Appendix $C$ of the Petroleum Supply Monthly. 
Figure 1. U.S. DistIllate Fuel OII Production

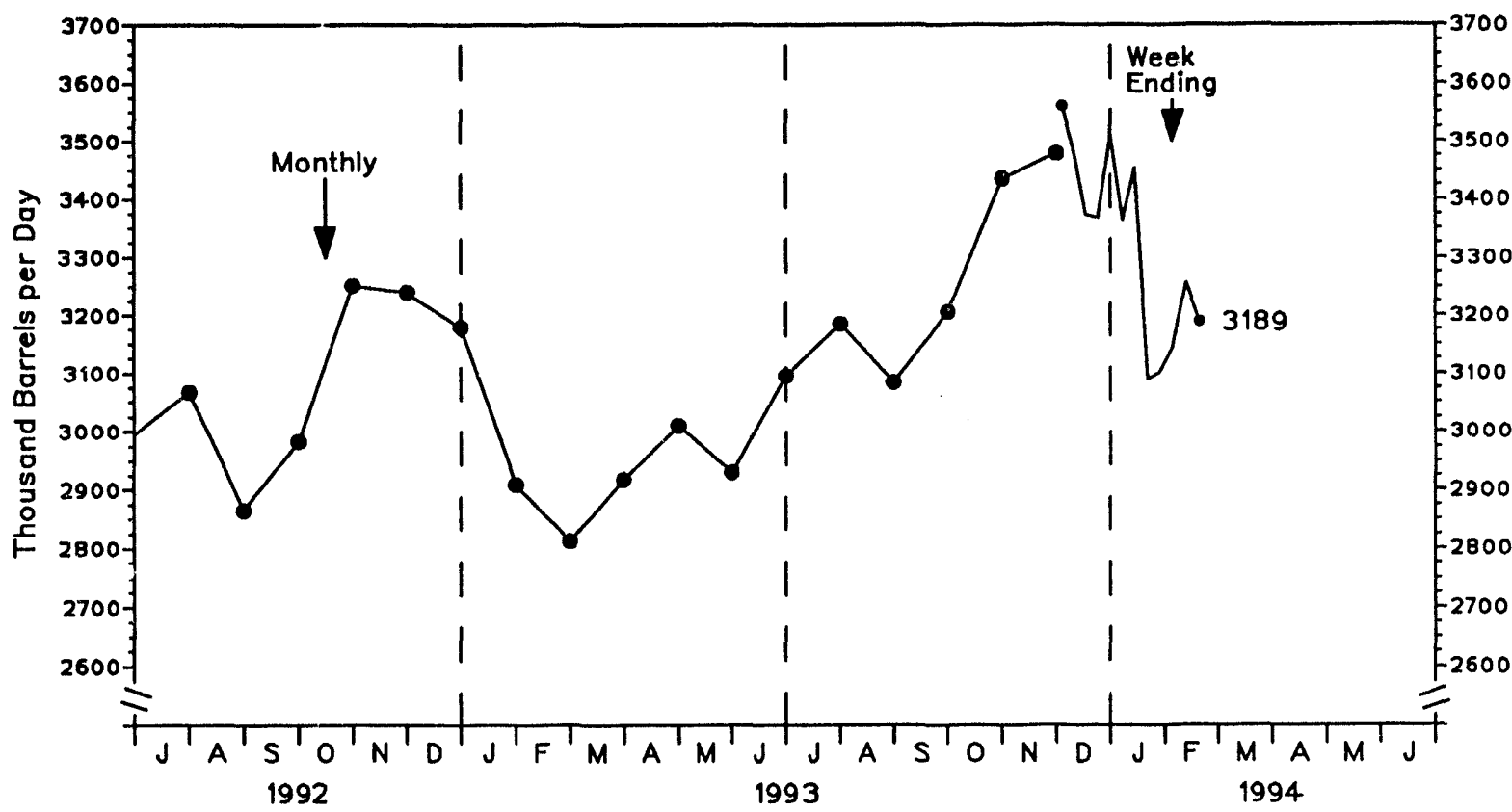

Source: - Monthly Data: 1992, EIA, Petroleum Supply Annual; 1993, Petroleum Supply Monthly. - Week-Ending Production: Estimates based on weekly data collected on Form EIA-800.

Figure 2. U.S. Distlllate Fuel OII Imports

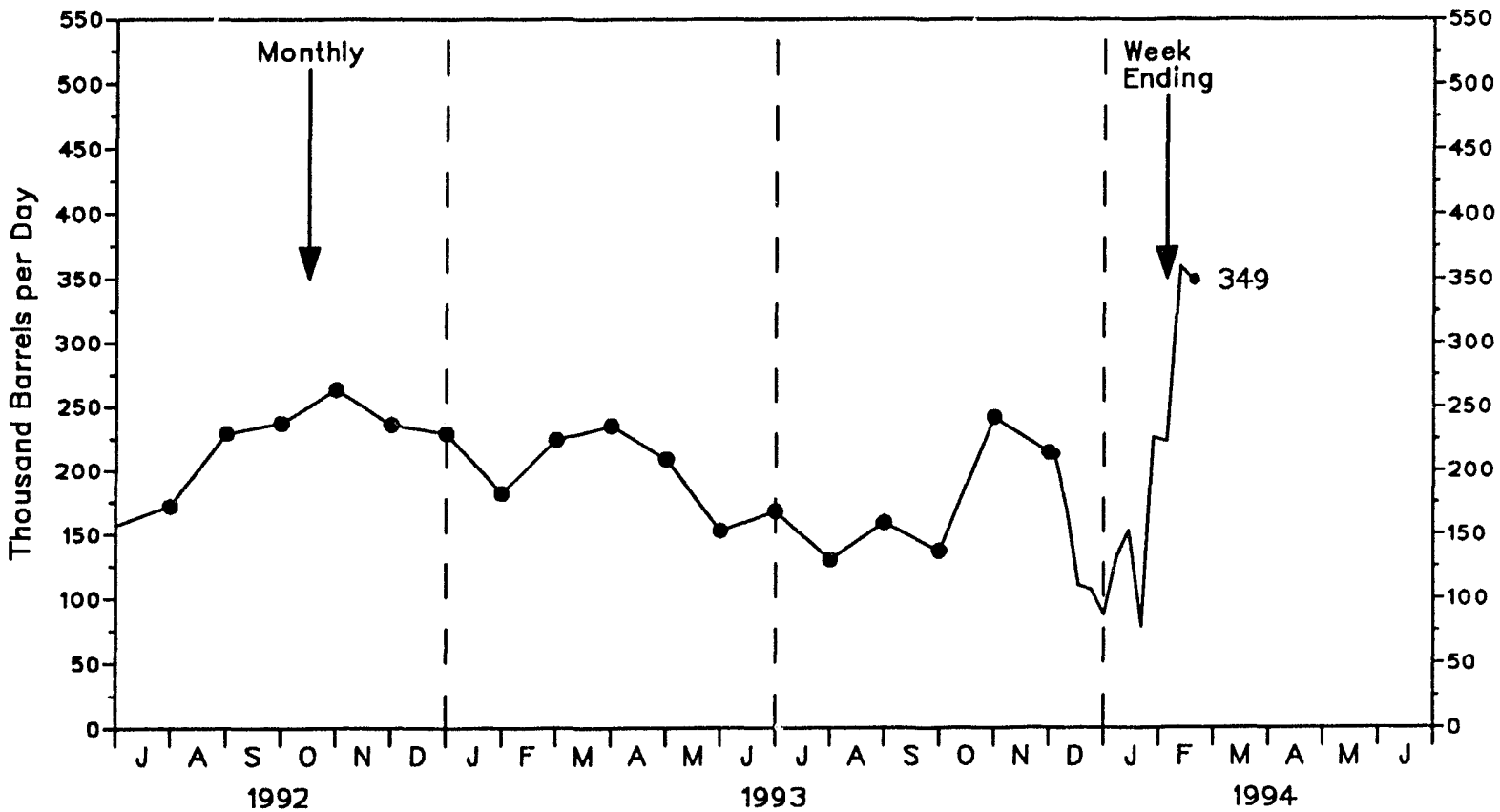

Source: - Monthly Data: 1992, ElA, Petroleum Supply Annual; 1993, Petroleum Supply Monthly. - Week-Ending Imports: Estimates based on weekly data collected on Form EIA.804. 
Figure 3. U.S. Distillate Fuel OII Stocks

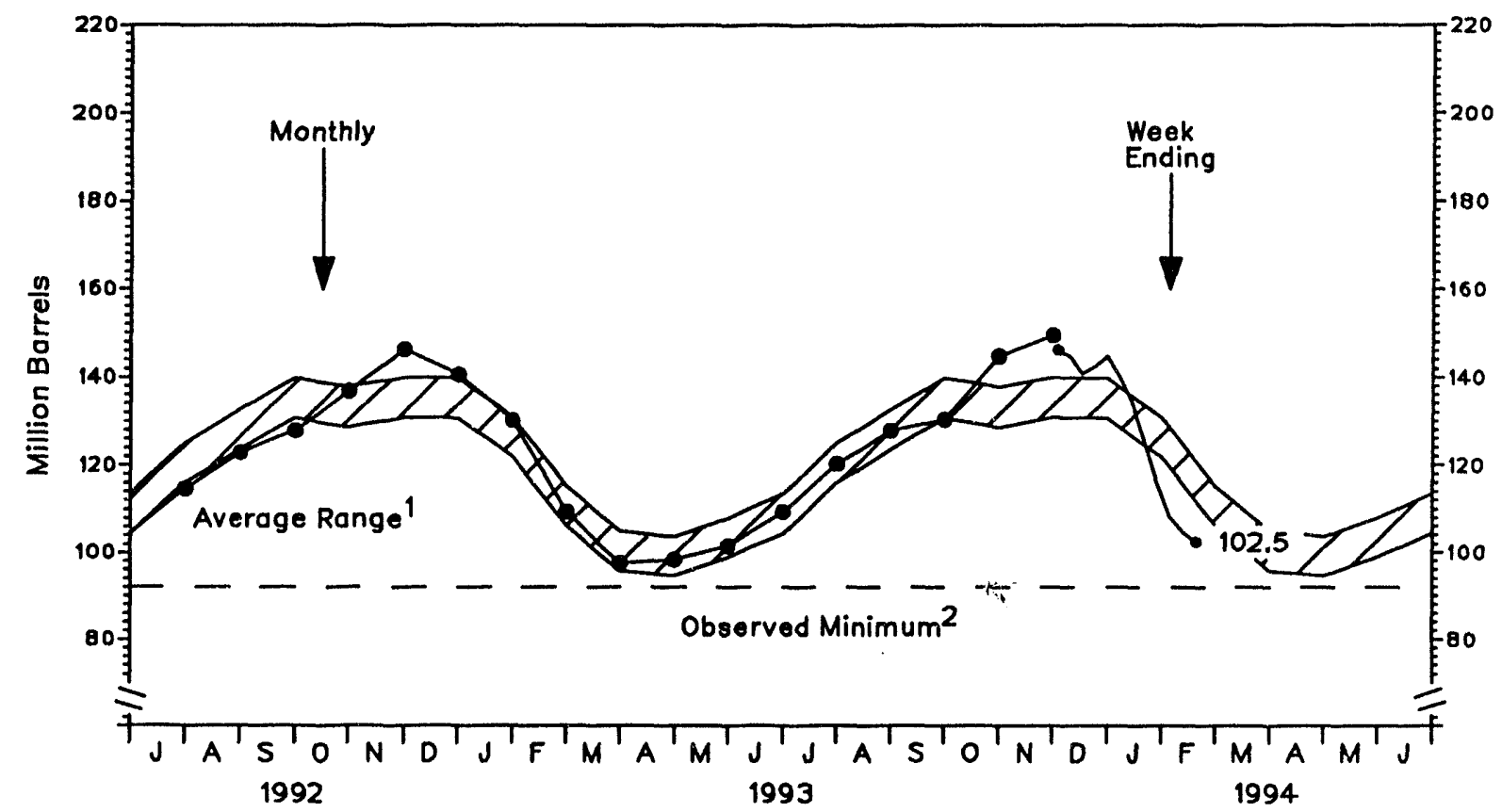

${ }^{1}$ Average level and width of average range are based on 3 years of monthly data: July 1990-June 1993. The seasonal pattern is based on 7 years of monthly data.

${ }^{2}$ The Observed Minimum for distillate fuel oil stocks in the last 36 month period was 92.1 million barrels, occurring in April 1992.

Source: - Data for Ranges and Seasonal Patterns: 1985-1992, Energy Information Administration (ElA), Petroleum Supply Annual; 1993, ElA. Petroleum Supply Monthly. - Monthly Data: 1992, ElA, Petroleum Supply Annual; 1993, Petroleum Supply Monthly. • Week-Ending Stocks: Estimates based on weekly data collected on Forms EIA-800, -801 , and -802 .

Figure 4. PADD I (East Coast) Distlllate Fuel Oll Stocks

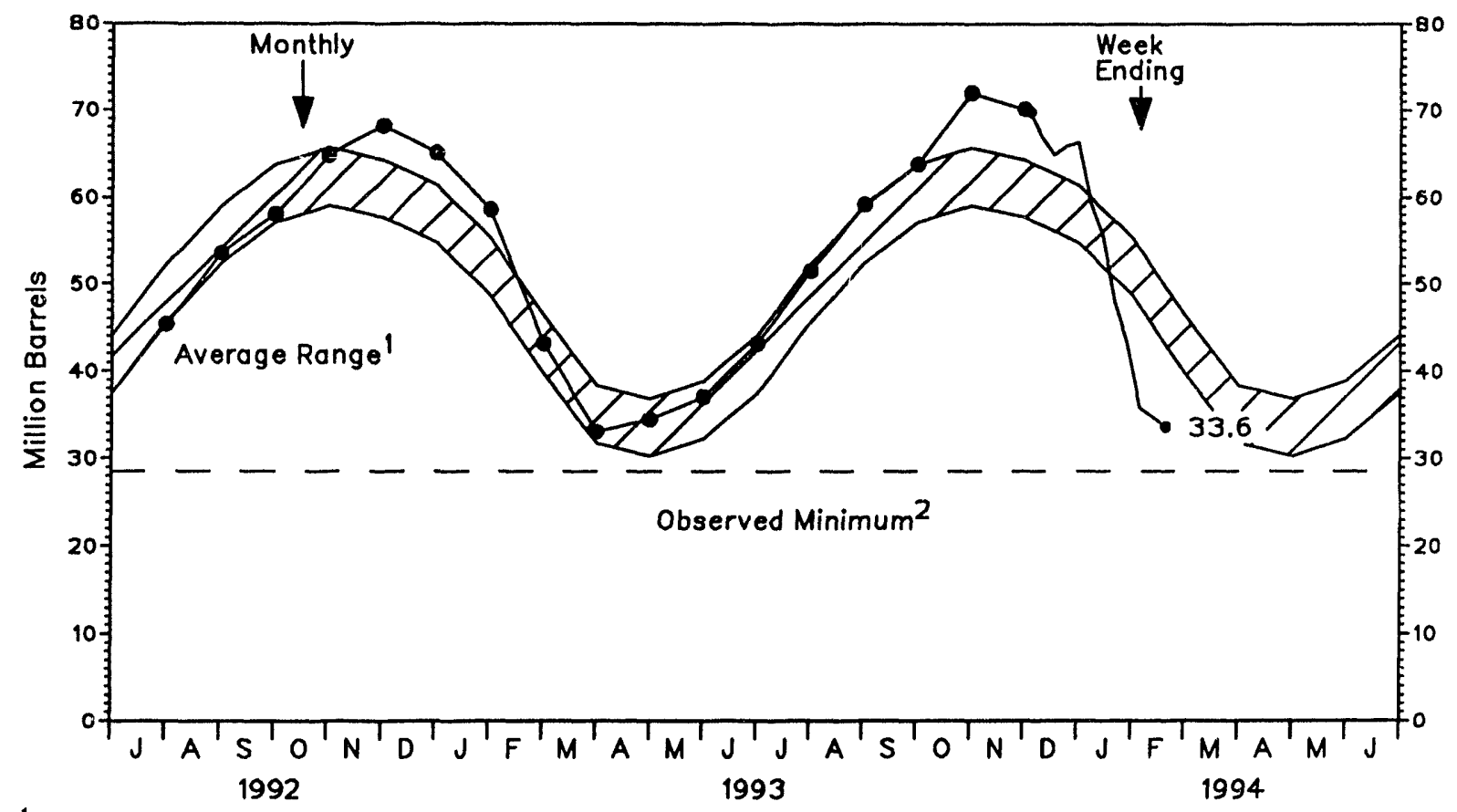

${ }^{1}$ Average level and width of average range are based on 3 years of monthly data: July 1990-June 1993. The seasonal pattern is based on 7 years of monthly data.

2 The Observed Minimum for distillate fuel oil stocks in the last 36 month period was 28.5 million barrels, occurring in April 1992.

Source: - Data for Ranges and Seasonal Patterns: 1985-1992, Energy Information Administration (ElA), Petroleum Supply Annual; 1993, ElA, Petroleum Supply Monthly. • Monthly Data: 1992, ElA, Petroleum Supply Annual; 1993, Petroleum Supply Monthly. - Week-Ending Stocks: Estimates based on weekly data collected on Forms EIA-800, -801, and -802 . 
Figure 5. PADD II (Mldwest) Distillate Fuel Oll Stocks

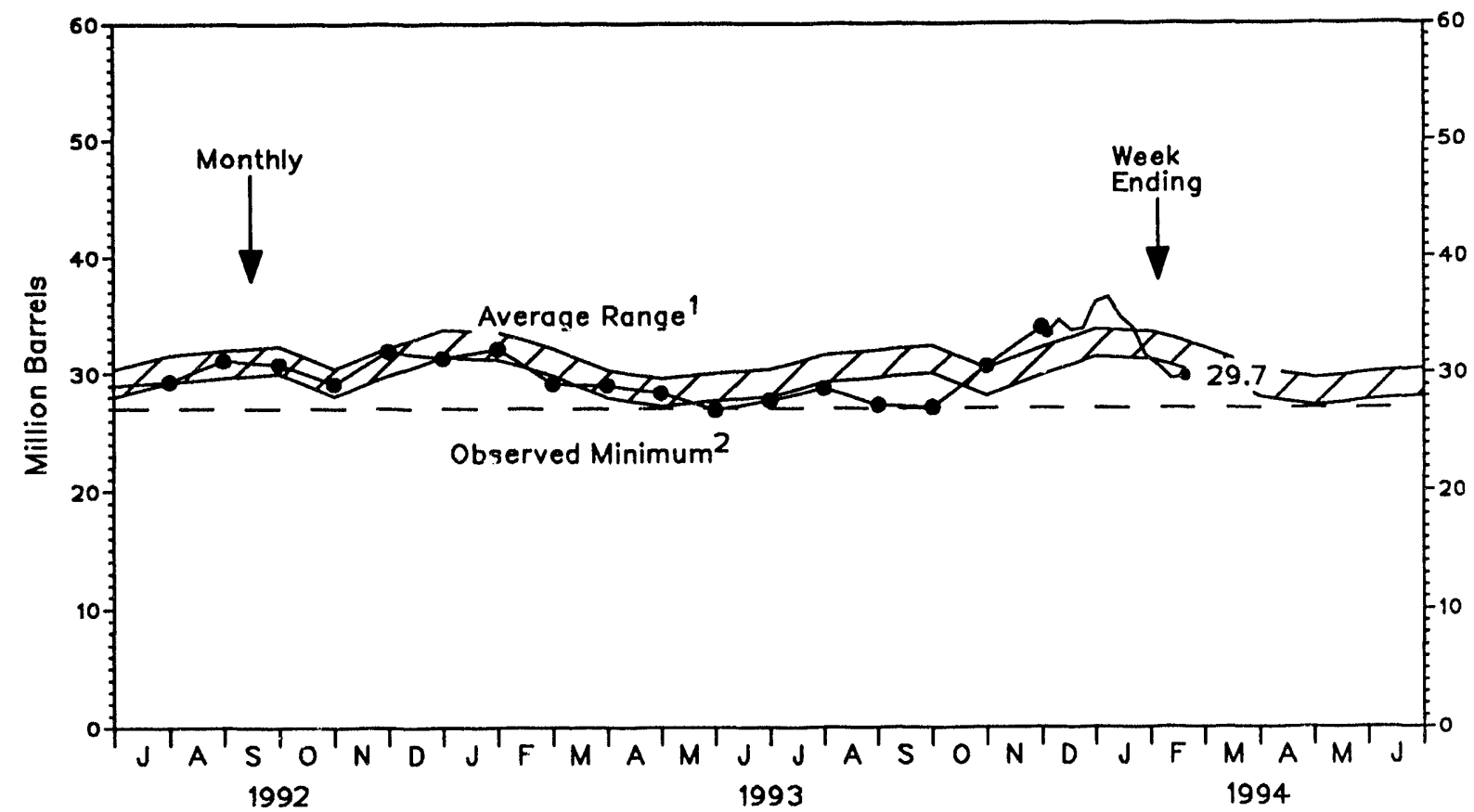

${ }^{1}$ Average level and width of average range are based on 3 years of monthly data: July 1990-June 1993. The seasonal pattern is based on 7 years of monthly data.

${ }_{2}^{2}$ The Observed Minimum for distillate fuel oil stocks in the last 36 month period was 26.9 million barrels, occurring in May 1993.

Source: - Data for Ranges and Seasonal Patterns: 1985-1992, Energy Information Administration (ElA), Petroleum Supply Annual; 1993, ElA, Petroleum Supply Monthly. - Monthly Data: 1992, ElA, Petroleum Supply Annual; 1993, Petroleum Supply Monthly. - Week-Ending Stocks: Estimates based on weekly data collected on Forms EIA-800, -801 , and -802 .

\section{Figure 6. PADD III (Gulf Coast) Distillate Fuel Oll Stocks}

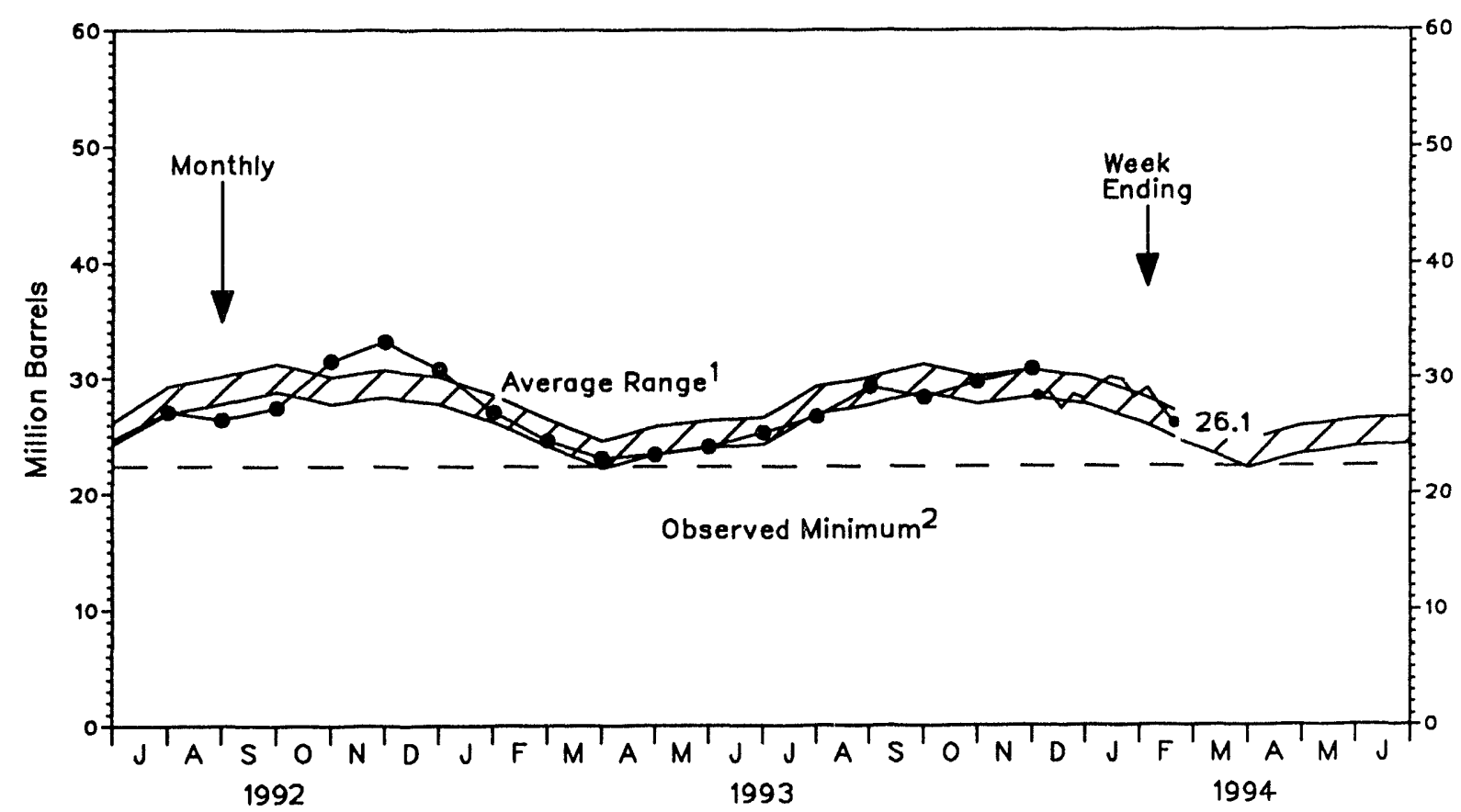

${ }^{1}$ Average level and width of average range are based on 3 years of monthly data: July 1990-June 1993. The seasonal pattern is based on 7 years of monthly data.

2 The Observed Minimum for distillate fuel oil stocks in the last 36 month period was 22.5 million barrels, occurring in February 1992.

Source: - Data for Ranges and Seasonal Patterns: 1985-1992, Energy Information Administration (ElA), Petroleum Supply Annual; 1993, ElA, Petroleum Supply Monthly. - Monthly Data: 1992, ElA, Petroleum Supply Annual; 1993, Petroleum Supply Monthly. - Week.Ending Stocks: Estimates based on weekly data collected on Forms EIA-800, -801 , and -802 .

Period Ending 02/18/94 Energy Information Administration/Winter Fuels Report 
Figure 7. PADD IV (Rocky Mountain) Distlllate Fuel Oll Stocks

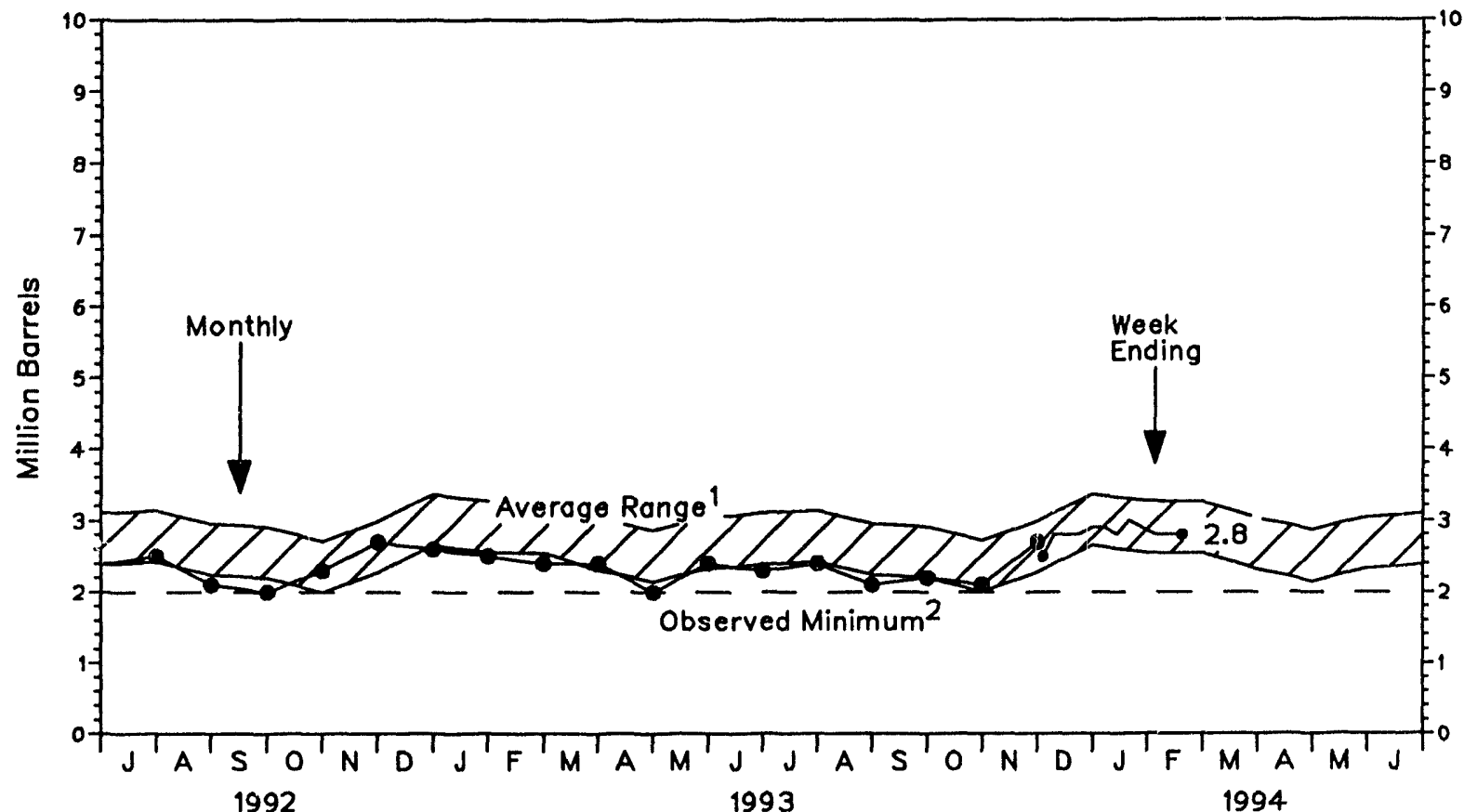

${ }^{1}$ Average level and width of average range are based on 3 years of mrsnthly data: July 1990-June 1993. The seasonal pattern is based on 7 years of monthly data.

2 The Observed Minimum for distillate fuel oil stocks in the last 36 month period was 2.0 million barrels, occurring in September 1992.

Source: - Data for Ranges and Seasonal Patterns: 1985-1992, Energy Information Administration (ElA), Petroleum Supply Annual; 1993, EIA, Petroleum Supply Monthly. • Monthly Data: 1992, ElA, Petroleum Supply Annual; 1993, Petroleum Supply Monthly. - Week-Ending Stocks: Estimates based on weekly data collected on Forms EIA-800, -801 , and -802 .

Figure 8. PADD V (West Coast) Distillate Fuel Oll Stocks

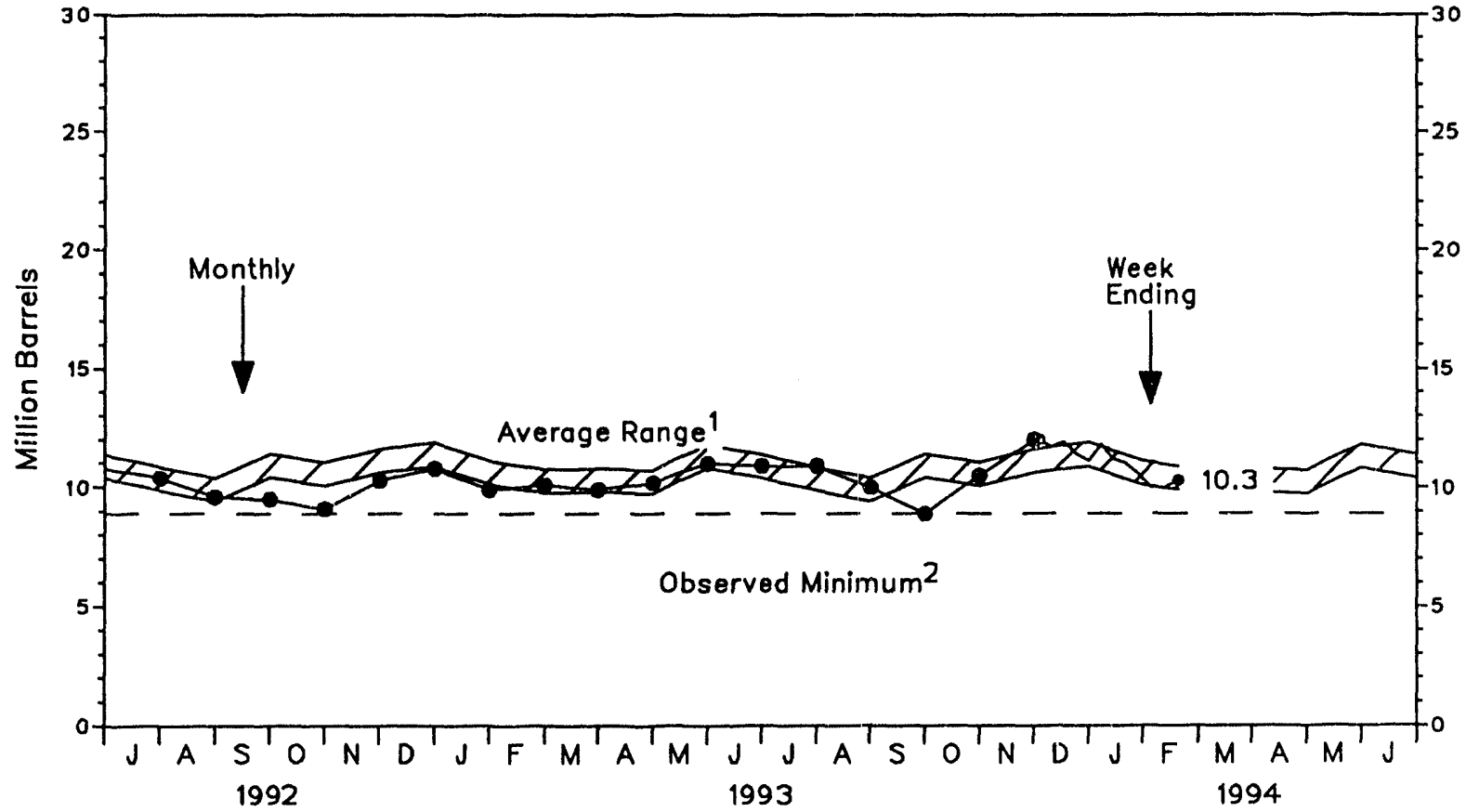

${ }^{1}$ Average level and width of average range are based on 3 years of monthly data: July 1990.June 1993. The seasonal pattern is based on 7 years of monthly data.

2 The Observed Minimum for distillate fuel oil stocks in the last 36 month period was 8.9 million barrels, occurring in September 1993.

Source: - Data for Ranges and Seasonal Patterns: 1985-1992, Energy Information Administration (ElA), Petroleum Supply Annual; 1993, ElA, Petroleum Supply Monthly. - Monthly Data: 1992, ElA, Petroleum Supply Annual; 1993, Petroleum Supply Monthly. - Week-Ending Stocks: Estimates based on weekly data collected on Forms EIA-800, -801 , and -802 . 


\section{Propane}

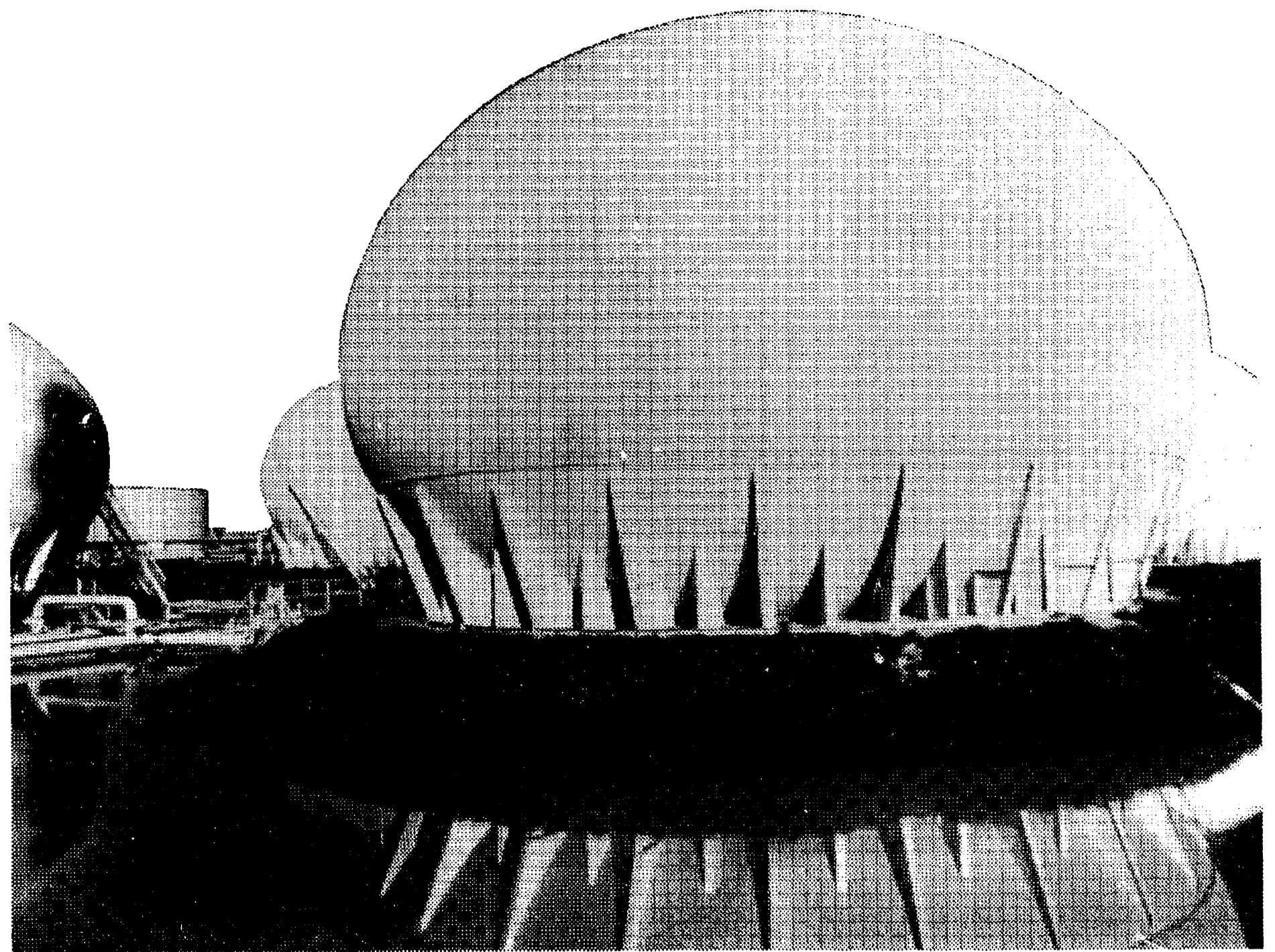

Spherical tanks are used to store liquefied petroleum gases under pressure. 
Table 2. Monthly and Weekly Net Production, Imports, and Stocks of Propane/Propylene by Petroleum Administration for Defense Districts (PADD) I, II, and III (Thousand Barrels per Day, Except Where Noted)

\begin{tabular}{|c|c|c|c|c|c|c|c|c|c|c|c|c|}
\hline District/Year & Jan & Feb & Mar & Apr & May & Jun & Jul & Aug & Sep & Oct & Nov & Dec \\
\hline \multicolumn{13}{|c|}{$\begin{array}{l}\text { Total U.S. } \\
\text { Net Production a }\end{array}$} \\
\hline 1991 & 920 & 923 & 912 & 900 & 922 & 906 & 901 & 891 & 905 & 902 & 930 & 964 \\
\hline 1992 & 949 & 955 & 940 & 961 & 977 & 978 & 964 & 946 & 931 & 933 & 964 & 977 \\
\hline 1993 & 965 & 959 & 971 & 973 & 942 & 958 & 956 & 945 & 956 & 953 & 964 & \\
\hline \multicolumn{13}{|l|}{ Imports } \\
\hline 1991 & 105 & 90 & 56 & 101 & 90 & 81 & 91 & 73 & 92 & 146 & 82 & 86 \\
\hline 1992 & 90 & 86 & 68 & 80 & 72 & 66 & 68 & 85 & 71 & 104 & 99 & 131 \\
\hline 1993 & 72 & 78 & 85 & 112 & 96 & 75 & 105 & 116 & 132 & 107 & 138 & \\
\hline \multicolumn{13}{|c|}{ Stocks (Million Barrels) } \\
\hline 1991 & 35.0 & 30.1 & 29.8 & 35.2 & 41.8 & 48.5 & 51.0 & 52.3 & 51.6 & 52.7 & 51.6 & 47.6 \\
\hline 1992 & 38.9 & 33.1 & 32.6 & 36.2 & 44.1 & 50.3 & 55.7 & 59.3 & 60.8 & 58.1 & 50.8 & 38.9 \\
\hline 1993 & 33.5 & 26.2 & 21.8 & 28.8 & 36.9 & 44.9 & 52.1 & 57.8 & 61.3 & 61.0 & 56.9 & \\
\hline \multicolumn{13}{|l|}{ Week Ending } \\
\hline $1993 / 1994$ & $\begin{array}{l}12 / 03 \\
E_{56.5}\end{array}$ & $\begin{array}{r}12 / 10 \\
E_{55.2}\end{array}$ & $\begin{array}{l}12 / 17 \\
E_{53.6}\end{array}$ & $\begin{array}{l}12 / 24 \\
E_{52.7}\end{array}$ & $\begin{array}{r}12 / 31 \\
E 49,7\end{array}$ & $\begin{array}{l}01 / 07 \\
\mathrm{E} 46.6\end{array}$ & $\begin{array}{l}01 / 14 \\
E 42.5\end{array}$ & $\begin{array}{l}01 / 21 \\
E_{37.8}\end{array}$ & $\begin{array}{l}01 / 28 \\
E_{33.8}\end{array}$ & $\begin{array}{l}02 / 04 \\
E_{301}\end{array}$ & $\begin{array}{l}02 / 11 \\
E_{27} 4\end{array}$ & $\begin{array}{r}02 / 18 \\
E_{24.8}\end{array}$ \\
\hline \multicolumn{13}{|c|}{$\begin{array}{l}\text { East Coast (PADD I) } \\
\text { Net Production }\end{array}$} \\
\hline 1991 & 55 & 54 & 56 & 47 & 54 & 52 & 50 & 47 & 49 & 48 & 50 & 58 \\
\hline 1992 & 60 & 60 & 60 & 56 & 52 & 60 & 56 & 54 & 54 & 63 & 63 & 65 \\
\hline 1993 & 57 & 55 & 53 & 53 & 52 & 59 & 56 & 54 & 56 & 60 & 55 & \\
\hline \multicolumn{13}{|l|}{ Week Ending } \\
\hline $1993 / 1994$ & $\begin{array}{l}12 / 03 \\
E 66\end{array}$ & $\begin{array}{l}12 / 10 \\
E 61\end{array}$ & $\begin{array}{l}12 / 17 \\
E 57\end{array}$ & $\begin{array}{l}12 / 24 \\
E_{55}\end{array}$ & $\begin{array}{l}12 / 31 \\
E 57\end{array}$ & $\begin{array}{l}01 / 07 \\
E_{49}\end{array}$ & $\begin{array}{l}01 / 14 \\
E_{64}\end{array}$ & $\begin{array}{l}01 / 21 \\
E_{44}\end{array}$ & $\begin{array}{l}01 / 28 \\
\varepsilon_{44}\end{array}$ & $E_{45}^{02 / 04}$ & $02 / 11$ & $\mathrm{E}_{62}^{02 / 18}$ \\
\hline \multicolumn{13}{|l|}{ Imports } \\
\hline 1991 & 24 & 17 & 18 & 16 & 7 & 15 & 3 & 4 & 22 & 13 & 18 & 26 \\
\hline 1992 & 23 & 27 & 19 & 14 & 13 & 16 & 8 & 11 & 15 & 12 & 27 & 22 \\
\hline 1993 & 21 & 23 & 16 & 23 & 4 & 17 & 8 & 4 & 18 & 14 & 22 & \\
\hline \multicolumn{13}{|l|}{ Weok Ending } \\
\hline $1993 / 1994$ & $\begin{array}{l}12 / 03 \\
15\end{array}$ & $\begin{array}{l}12 / 10 \\
64\end{array}$ & $\begin{array}{l}12 / 17 \\
E_{33}\end{array}$ & $12 / 24$ & $\begin{array}{l}12 / 31 \\
E 12\end{array}$ & $\begin{array}{l}01 / 07 \\
E 13\end{array}$ & $\mathrm{E}_{86}^{01 / 14}$ & $\begin{array}{l}01 / 21 \\
E 67\end{array}$ & $\begin{array}{l}01 / 28 \\
E_{50}\end{array}$ & $\begin{array}{l}02 / 04 \\
E_{14}\end{array}$ & $02 / 11$ & $\begin{array}{l}02 / 18 \\
E_{82}\end{array}$ \\
\hline \multicolumn{13}{|c|}{ Stocks (Million Barrels) } \\
\hline 1991 & 4.1 & 3.5 & 3.8 & 4.2 & 4.1 & 4.2 & 3.9 & 3.3 & 3.6 & 4.1 & 4.2 & 4.1 \\
\hline 1992 & 2.9 & 2.6 & 2.4 & 2.4 & 2.7 & 3.1 & 3.5 & 4.0 & 4.3 & 4.3 & 4.7 & 3.7 \\
\hline 1993 & 3.2 & 2.0 & 1.6 & 2.1 & 2.5 & 3.8 & 4.3 & 4.2 & 4.4 & 4.5 & 4.2 & \\
\hline \multicolumn{13}{|l|}{ Week Ending } \\
\hline $1993 / 1994$ & $\begin{array}{l}12 / 03 \\
E 4.1\end{array}$ & $\begin{array}{l}12 / 10 \\
E_{4,1}\end{array}$ & $\begin{array}{r}12 / 17 \\
E_{4.4}\end{array}$ & $\begin{array}{l}12 / 24 \\
E_{4.4}\end{array}$ & $\begin{array}{l}12 / 31 \\
E_{3.7}\end{array}$ & $\begin{array}{l}01 / 07 \\
E_{3.2}\end{array}$ & $\begin{array}{l}01 / 14 \\
E_{2,8}\end{array}$ & $\begin{array}{r}01 / 21 \\
E_{2.3}\end{array}$ & $\begin{array}{r}01 / 28 \\
E 1.8\end{array}$ & $\begin{array}{l}02 / 04 \\
E 1.5\end{array}$ & $\begin{aligned} 02 / 11 \\
E_{1.6}\end{aligned}$ & $\begin{array}{r}02 / 18 \\
E_{1.8}\end{array}$ \\
\hline
\end{tabular}


Table 2. Monthly and Weekly Net Production, Imports, and Stocks of Propane/Propylene by Petroleum Administration for Defense Distrlcts (PADD) I, II, and III (Continued)

(Thousanc Barrels per Day Except Where Noted)

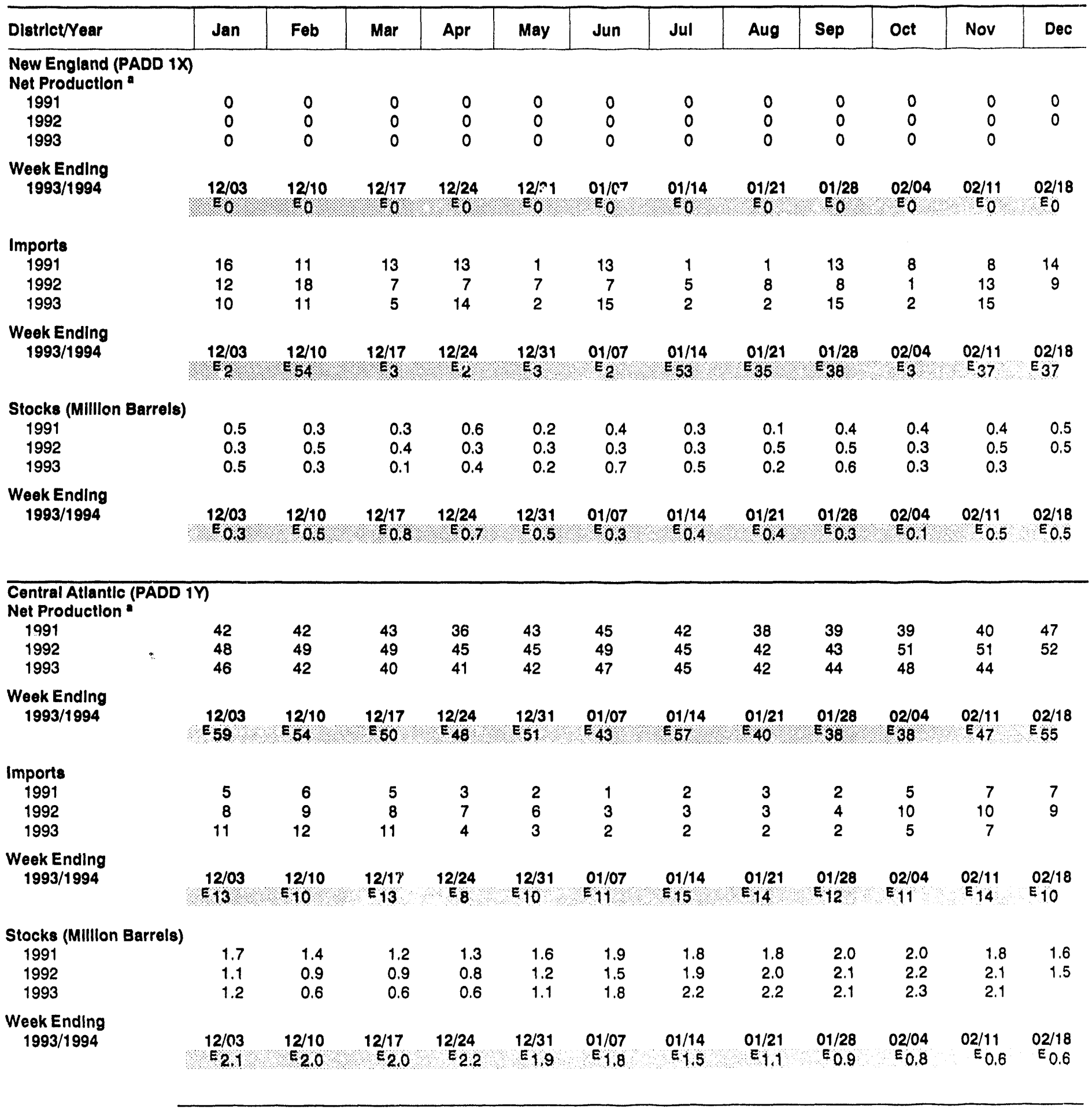

See footnotes at end of table. 
Table 2. Monthly and Weekly Net Production, Imports, and Stocks of Propane/Propylene by Petroleum Administration for Defense Districts (PADD) I, II, and III (Continued)

(Thousand Barrels per Day Except Where Noted)

\begin{tabular}{|c|c|c|c|c|c|c|c|c|c|c|c|c|}
\hline District/Year & Jan & Feb & Mar & Apr & May & Jun & Jul & Aug & Sep & Oct & Nov & Dec \\
\hline \multicolumn{13}{|c|}{$\begin{array}{l}\text { Lower Atlantic (PADD 1Z) } \\
\text { Net Production }\end{array}$} \\
\hline 1991 & 12 & 11 & 13 & 12 & 12 & 7 & 8 & 10 & 10 & 10 & 10 & 11 \\
\hline 1992 & 12 & 11 & 11 & 11 & 7 & 11 & 11 & 11 & 11 & 12 & 13 & 13 \\
\hline 1993 & 12 & 13 & 14 & 12 & 9 & 12 & 11 & 12 & 12 & 12 & 11 & \\
\hline \multicolumn{13}{|l|}{ Week Ending } \\
\hline $1993 / 1994$ & $\begin{array}{l}12 / 03 \\
E 7\end{array}$ & $\begin{array}{l}12 / 10 \\
67\end{array}$ & $12 / 17$ & $12 / 24$ & $12 / 31$ & $01 / 07$ & $01 / 14$ & $01 / 21$ & $01 / 28$ & $\begin{array}{l}02 / 04 \\
87\end{array}$ & $02 / 11$ & $\begin{array}{c}02 / 18 \\
E_{6}\end{array}$ \\
\hline \multicolumn{13}{|l|}{ Imports } \\
\hline 1991 & 3 & 0 & 0 & 0 & 4 & 0 & 0 & 0 & 7 & 0 & 4 & 5 \\
\hline 1992 & 3 & 0 & 3 & 0 & 0 & 6 & 0 & 0 & 3 & 0 & 4 & 3 \\
\hline 1993 & 0 & 0 & 0 & 5 & 0 & 0 & 5 & 0 & 0 & 6 & 0 & \\
\hline \multicolumn{13}{|l|}{ Week Ending } \\
\hline $1993 / 1994$ & $12 / 03$ & $12 / 10$ & $\mathbb{E}^{12 / 17}$ & $12 / 24$ & $12 / 31$ & $01 / 07$ & $01 / 14$ & E $1 / 21$ & $01 / 28$ & $02 / 04$ & $02 / 11$ & $E_{36}^{02 / 18}$ \\
\hline \multicolumn{13}{|c|}{ Stocks (Million Barrels) } \\
\hline 1991 & 1.9 & 1.8 & 2.3 & 2.3 & 2.3 & 1.9 & 1.8 & 1.4 & 1.2 & 1.7 & 2.0 & 2.0 \\
\hline 1992 & 1.4 & 1.1 & 1.2 & 1.2 & 1.1 & 1.3 & 1.2 & 1.5 & 1.7 & 1.9 & 2.1 & 1.6 \\
\hline 1993 & 1.5 & 1.0 & 0.9 & 1.1 & 1.3 & 1.4 & 1.6 & 1.7 & 1.7 & 1.9 & 1.8 & \\
\hline \multicolumn{13}{|l|}{ Week Ending } \\
\hline $1993 / 1994$ & $12 / 03$ & $12 / 10$ & $12 / 17$ & $12 / 24$ & $12 / 31$ & $01 / 07$ & $\begin{array}{l}01 / 14 \\
00.9\end{array}$ & $01 / 21$ & $\begin{array}{l}01 / 28 \\
E_{0.6}\end{array}$ & $\begin{array}{l}02 / 04 \\
E 0.6\end{array}$ & $\begin{array}{l}02 / 11 \\
E_{0.5}\end{array}$ & $\begin{array}{l}02 / 18 \\
E_{0.7}\end{array}$ \\
\hline \multicolumn{13}{|c|}{$\begin{array}{l}\text { Midwest (PADD II) } \\
\text { Net Production : }\end{array}$} \\
\hline 1991 & 217 & 229 & 219 & 214 & 215 & 208 & 214 & 211 & 210 & 213 & 217 & 231 \\
\hline 1992 & 231 & 234 & 216 & 210 & 214 & 223 & 214 & 223 & 216 & 212 & 227 & 222 \\
\hline 1993 & 228 & 212 & 222 & 225 & 209 & 217 & 207 & 212 & 212 & 213 & 222 & \\
\hline \multicolumn{13}{|l|}{ Week Ending } \\
\hline $1993 / 1994$ & $\begin{array}{c}12 / 03 \\
250\end{array}$ & $\begin{array}{l}12 / 10 \\
E 230\end{array}$ & $\begin{array}{l}12 / 17 \\
E 251\end{array}$ & $\begin{array}{c}12 / 24 \\
E 242\end{array}$ & $\begin{array}{l}12 / 31 \\
241\end{array}$ & $\begin{array}{l}01 / 07 \\
\mathbf{2}_{40}\end{array}$ & E $201 / 14$ & $\begin{array}{c}01 / 21 \\
E_{240}\end{array}$ & $\begin{array}{c}01 / 28 \\
E_{233}\end{array}$ & $\begin{array}{r}02 / 04 \\
247\end{array}$ & $\begin{array}{c}02 / 11 \\
E_{240}\end{array}$ & $E_{229}^{02 / 18}$ \\
\hline \multicolumn{13}{|l|}{ Imports } \\
\hline 1991 & 63 & 59 & 33 & 40 & 44 & 41 & 34 & 47 & 49 & 52 & 45 & 53 \\
\hline 1992 & 59 & 55 & 47 & 43 & 42 & 40 & 32 & 45 & 43 & 60 & 61 & 74 \\
\hline 1993 & 44 & 43 & 47 & 41 & 41 & 29 & 45 & 48 & 45 & 58 & 60 & \\
\hline \multicolumn{13}{|l|}{ Week Ending } \\
\hline $1993 / 1994$ & $E_{40}^{12 / 03}$ & $E_{68}^{12 / 10}$ & $12 / 17$ & $12 / 24$ & $\begin{array}{l}12 / 31 \\
2\end{array}$ & $\begin{array}{l}01 / 07 \\
688\end{array}$ & $\begin{array}{l}01 / 14 \\
78\end{array}$ & $\begin{array}{l}01 / 21 \\
E_{56}\end{array}$ & $\begin{array}{l}01 / 28 \\
E_{64}\end{array}$ & $\begin{array}{l}02 / 04 \\
E_{54}\end{array}$ & $E_{50}$ & $E_{40}^{02 / 18}$ \\
\hline \multicolumn{13}{|c|}{ Stccks (Million Barrels) } \\
\hline 1991 & 12.9 & 11.1 & 11.7 & 13.8 & 17.1 & 20.2 & 21.8 & 23.3 & 22.9 & 22.6 & 20.3 & 17.7 \\
\hline 1992 & 14.3 & 12.9 & 13.4 & 15.4 & 18.4 & 20.9 & 23.4 & 24.5 & 24.6 & 21.6 & 16.3 & 11.6 \\
\hline 1993 & 10.7 & 7.7 & 7.4 & 9.9 & 12.7 & 15.5 & 18.4 & 20.9 & 22.7 & 21.5 & 20.4 & \\
\hline \multicolumn{13}{|l|}{ Week Ending } \\
\hline $1993 / 1994$ & $\begin{array}{l}12 / 03 \\
E / 9.4\end{array}$ & $\begin{array}{l}12 / 10 \\
19,3\end{array}$ & $12 / 17$ & 12/24 & $\begin{array}{c}12 / 31 \\
17,6\end{array}$ & $01 / 07$ & $01 / 14$ & $\begin{array}{l}01 / 21 \\
E_{13}, 4\end{array}$ & $\begin{array}{l}01 / 28 \\
\varepsilon_{11,6}\end{array}$ & $\begin{array}{l}02 / 04 \\
E_{10.6}\end{array}$ & $\begin{array}{c}02 / 11 \\
E_{9.9}\end{array}$ & $\begin{array}{l}02 / 18 \\
E_{9.1}\end{array}$ \\
\hline
\end{tabular}

See footnotes at end of table. 
Table 2. Monthly and Weekly Net Production, Imports, and Stocks of Propane/Propylene by Petroleum Administration for Defense Districts (PADD) I, II, and III (Continued)

(Thousand Barrels per Day Except Where Noted)

\begin{tabular}{|c|c|c|c|c|c|c|c|c|c|c|c|c|}
\hline District/Year & Jan & Feb & Mar & Apr & May & Jun & Jul & Aug & Sep & Oct & Nov & Dec \\
\hline \multicolumn{13}{|c|}{$\begin{array}{l}\text { Gulf Coast (PADD III) } \\
\text { Net Production " }\end{array}$} \\
\hline $\begin{array}{l}1991 \\
1992 \\
1993\end{array}$ & $\begin{array}{l}545 \\
560 \\
577\end{array}$ & $\begin{array}{l}544 \\
559 \\
590\end{array}$ & $\begin{array}{l}535 \\
563 \\
590\end{array}$ & $\begin{array}{l}539 \\
584 \\
593\end{array}$ & $\begin{array}{l}549 \\
602 \\
583\end{array}$ & $\begin{array}{l}543 \\
590 \\
585\end{array}$ & $\begin{array}{l}539 \\
587 \\
595\end{array}$ & $\begin{array}{l}533 \\
569 \\
581\end{array}$ & $\begin{array}{l}553 \\
559 \\
585\end{array}$ & $\begin{array}{l}540 \\
558 \\
580\end{array}$ & $\begin{array}{l}562 \\
569 \\
582\end{array}$ & $\begin{array}{l}575 \\
586\end{array}$ \\
\hline \multicolumn{13}{|l|}{ Week Ending } \\
\hline $1993 / 1994$ & $\begin{array}{r}12 / 03 \\
564\end{array}$ & $\begin{array}{r}12 / 10 \\
551\end{array}$ & $12 / 17$ & $\begin{array}{c}12 / 24 \\
E_{563}\end{array}$ & $\begin{array}{c}12 / 31 \\
566\end{array}$ & $\begin{array}{l}01 / 07 \\
5 / 48\end{array}$ & $\begin{array}{c}01 / 14 \\
503\end{array}$ & $\begin{array}{l}01 / 21 \\
626\end{array}$ & $\begin{array}{l}01 / 28 \\
554\end{array}$ & $\begin{array}{r}02 / 04 \\
E_{553}\end{array}$ & $\begin{array}{r}02 / 11 \\
E 495\end{array}$ & $\begin{array}{l}02 / 18 \\
E_{501}\end{array}$ \\
\hline \multicolumn{13}{|l|}{ Imports } \\
\hline 1991 & 7 & 7 & 0 & 41 & 36 & 22 & 51 & 16 & 15 & 73 & 8 & 0 \\
\hline 1992 & 0 & 0 & 0 & 20 & 14 & 7 & 26 & 28 & 10 & 29 & 7 & 29 \\
\hline 1993 & 0 & 7 & 19 & 45 & 48 & 27 & 50 & 61 & 65 & 31 & 50 & \\
\hline \multicolumn{13}{|l|}{ Week Ending } \\
\hline $1993 / 1994$ & $12 / 03$ & $12 / 10$ & $12 / 17$ & $12 / 24$ & $12 / 31$ & $01 / 07$ & $E_{60}^{01 / 14}$ & $\begin{array}{l}01 / 21 \\
E_{4}\end{array}$ & $01 / 28$ & $\begin{array}{l}02 / 04 \\
E_{6}\end{array}$ & $\begin{array}{c}02 / 11 \\
E_{8}\end{array}$ & $\begin{array}{l}02 / 18 \\
E_{8}\end{array}$ \\
\hline \multicolumn{13}{|c|}{ Stocks (Million Barrels) } \\
\hline 1991 & 17.2 & 14.8 & 13.6 & 16.5 & 19.7 & 22.9 & 23.9 & 23.9 & 22.9 & 23.6 & 24.7 & 23.9 \\
\hline 1992 & 20.5 & 16.5 & 15.7 & 17.4 & 21.6 & 24.7 & 27.0 & 28.7 & 29.8 & 29.9 & 27.8 & 22.1 \\
\hline 1993 & 18.8 & 15.9 & 12.2 & 16.2 & 20.7 & 24.3 & 28.0 & 31.0 & 32.3 & 33.0 & 30.5 & \\
\hline \multicolumn{13}{|l|}{ Week Ending } \\
\hline $1993 / 1994$ & $\begin{array}{l}12 / 03 \\
E_{316}\end{array}$ & $\begin{array}{l}12 / 10 \\
E_{30,4}\end{array}$ & $\begin{array}{l}12 / 17 \\
E_{29,1}\end{array}$ & $\begin{array}{l}12 / 24 \\
E 28.5\end{array}$ & $\begin{array}{l}12 / 31 \\
E_{27,1}\end{array}$ & $\begin{array}{l}01 / 07 \\
E_{25,4}\end{array}$ & $\begin{array}{l}01 / 14 \\
\mathrm{E}_{23.2}\end{array}$ & $\begin{array}{l}01 / 21 \\
\mathrm{E}_{21,2}\end{array}$ & $\begin{array}{l}01 / 28 \\
E_{19.5}\end{array}$ & $\begin{array}{l}02 / 04 \\
E_{17.3}\end{array}$ & $\begin{array}{l}02 / 11 \\
E_{15.2}\end{array}$ & $\begin{array}{l}02 / 18 \\
E_{13.2}\end{array}$ \\
\hline
\end{tabular}

Net production equals gross production minus input. Negative production will occur when the amount of product produced during the month is less than the amount of that same product reprocessed (input) or reclassified to become another product during the same month.

E=Estimated data.

Note: - This table presents weekly data, derived from a cut-off sample of refineries and fractionators that produce propane and from companies that import or

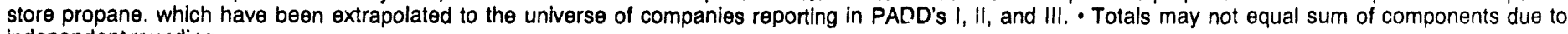
independent rounding.

Source: Energy Information Administration (EIA), Monthly Petroleum Supply Reporting System and data collected on Form ElA-807, "Propane Telephone Survey." Magnitudes of revisions to monthly data are published in Appendix C of the Petroleum Supply Monthly. 
Figure 9. U.S. Propane Production

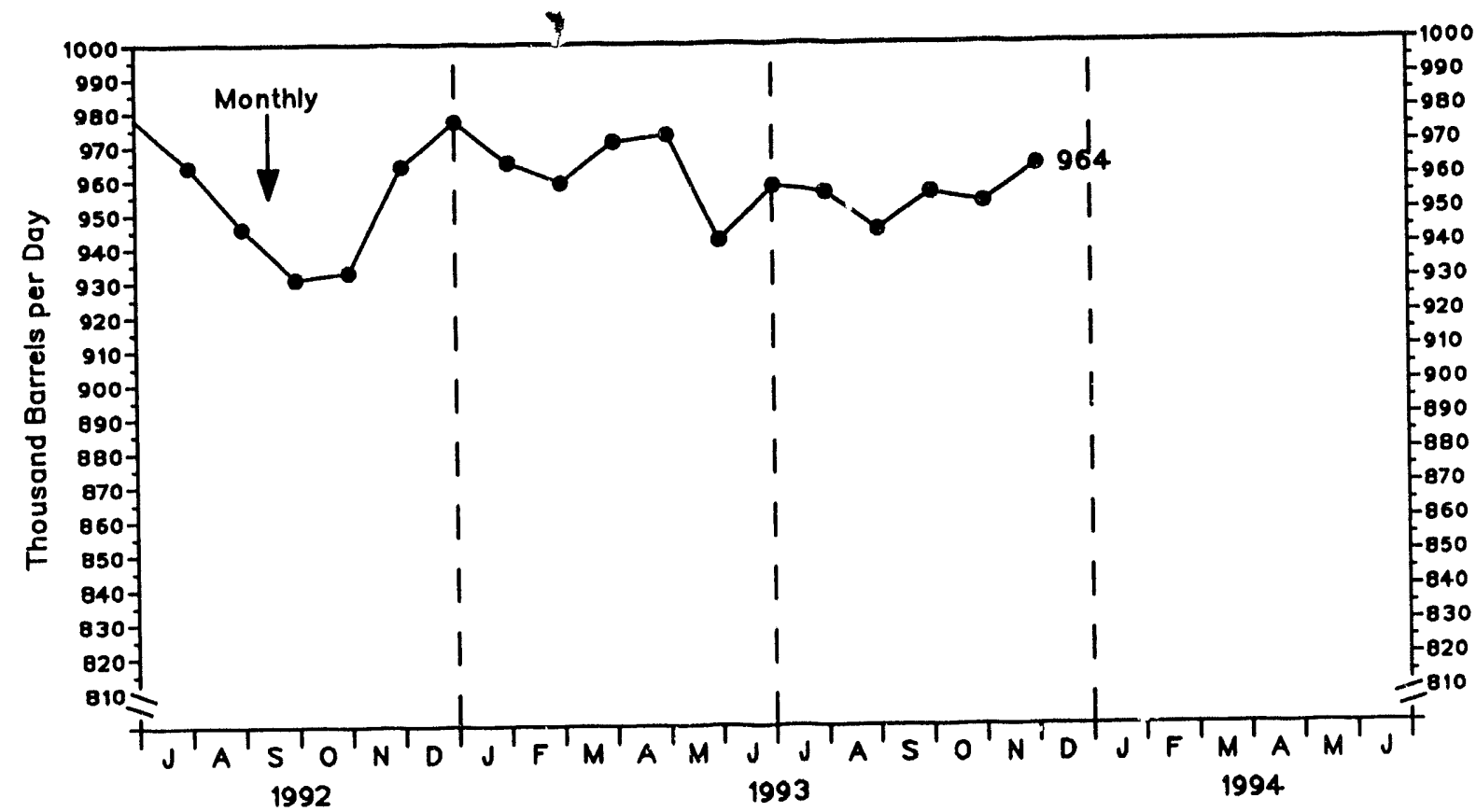

Source: 1992, EIA, Petroleum Supply Annual; 1993, EIA, Petroleum Supply Monthly.

Figure 10. U.S. Propane Imports

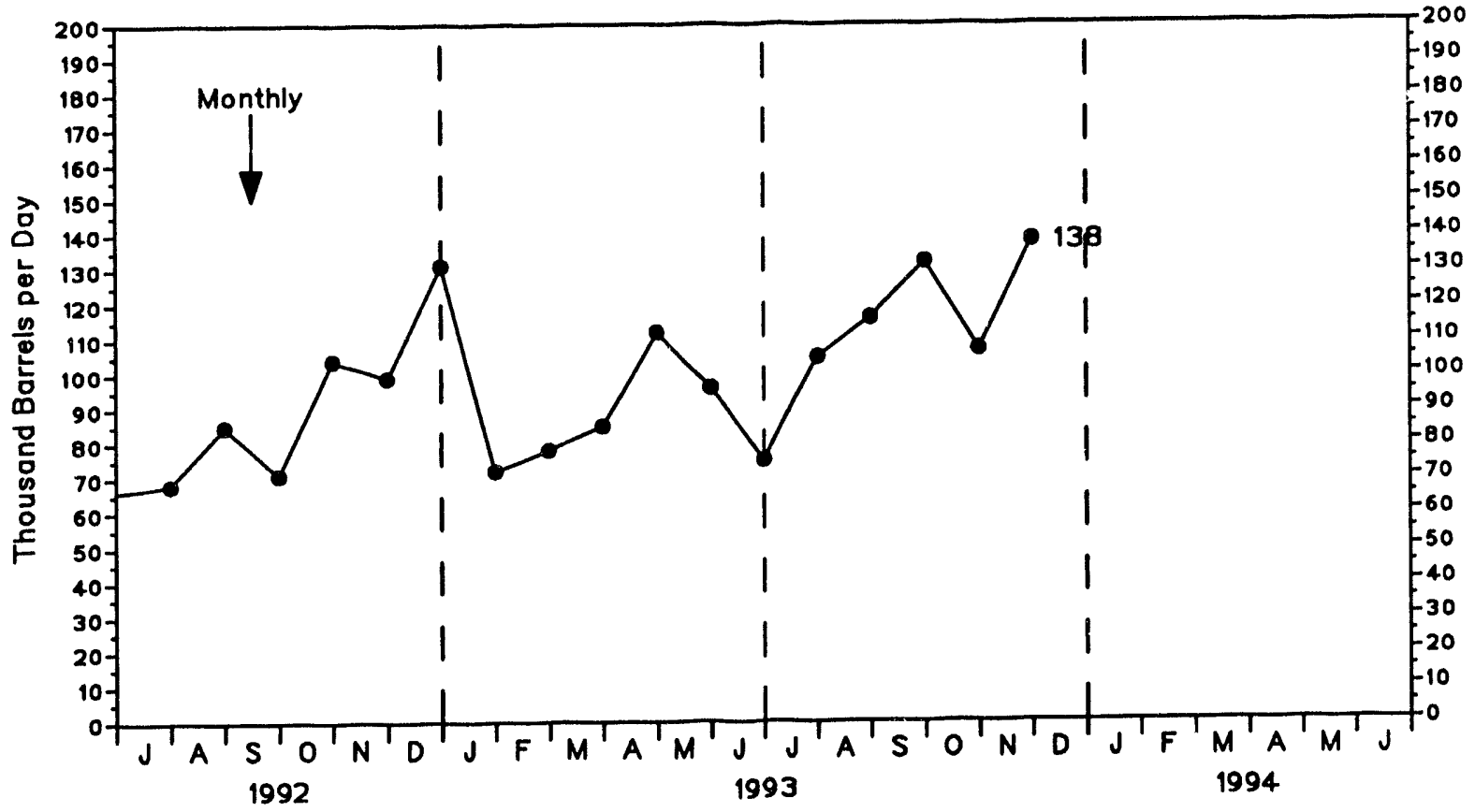

Source: 1992, ElA, Petroloum Supply Annual; 1993, EIA, Petroleum Supply Monthly. 
Figure 11. U.S. Propane Stocks

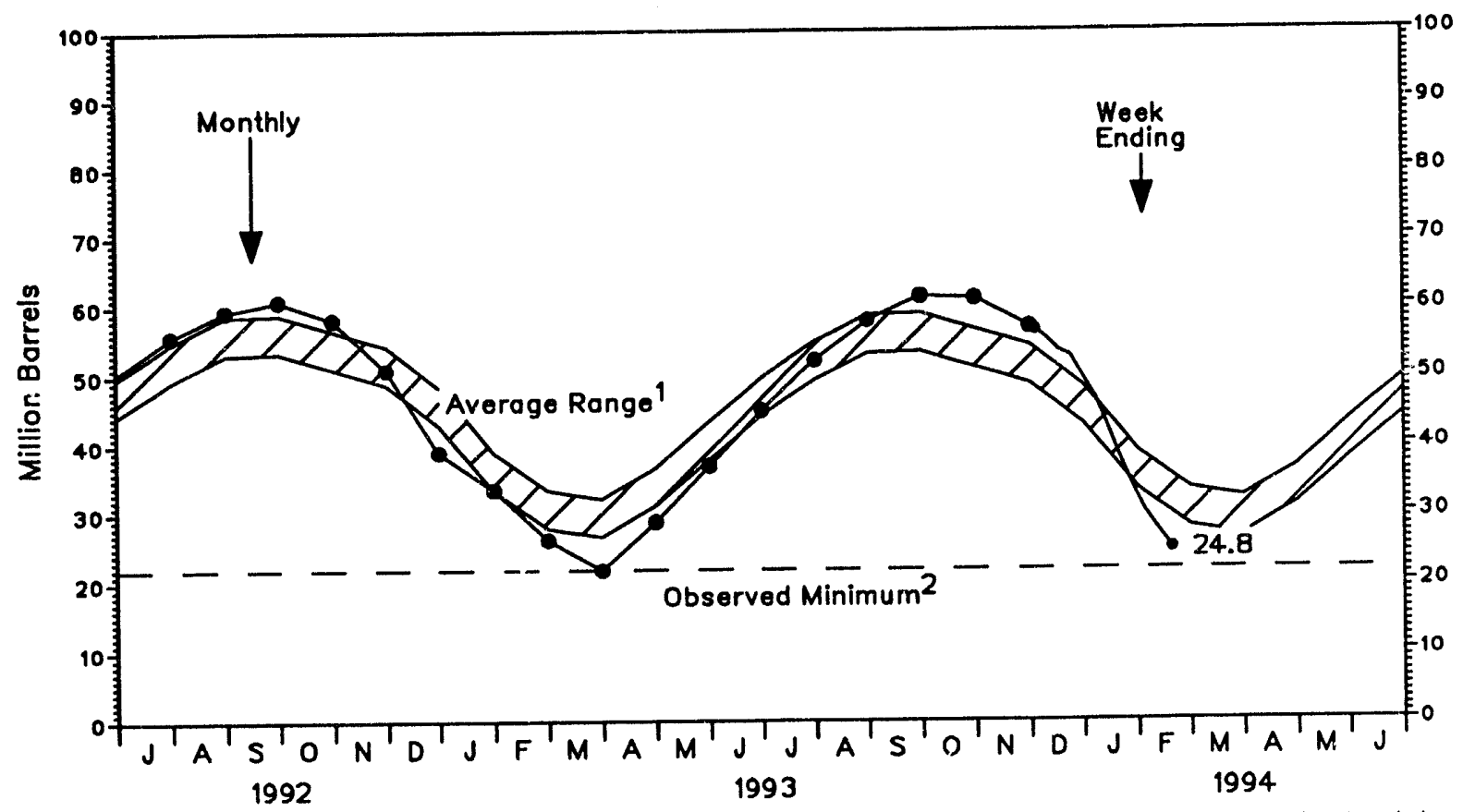

1 Average level and width of average range are based on 3 years of monthly data: July 1990-June 1993. The seasonal pattern is based on 7 years of monthly data.

2 The Observed Minimum for propane stocks in the last 36 month period was 21.8 million barrels, occurring in March 1993.

Source: - Data for Ranges and Seasonal Patterns: 1985-1992, Energy Information Administration (EIA), Petroleum Supply Annual; 1993, ElA, Petroleum Supply Monthly. - Monthly Data: 1992, EIA, Petroleum Supply Annual; 1993, ElA, Petroleum Supply Monthly. - Week-Ending Stocks: Estimates based on data from Table $\mathrm{H} 1$.

\section{Figure 12. PADD I (East Coast) Propane Stocks}

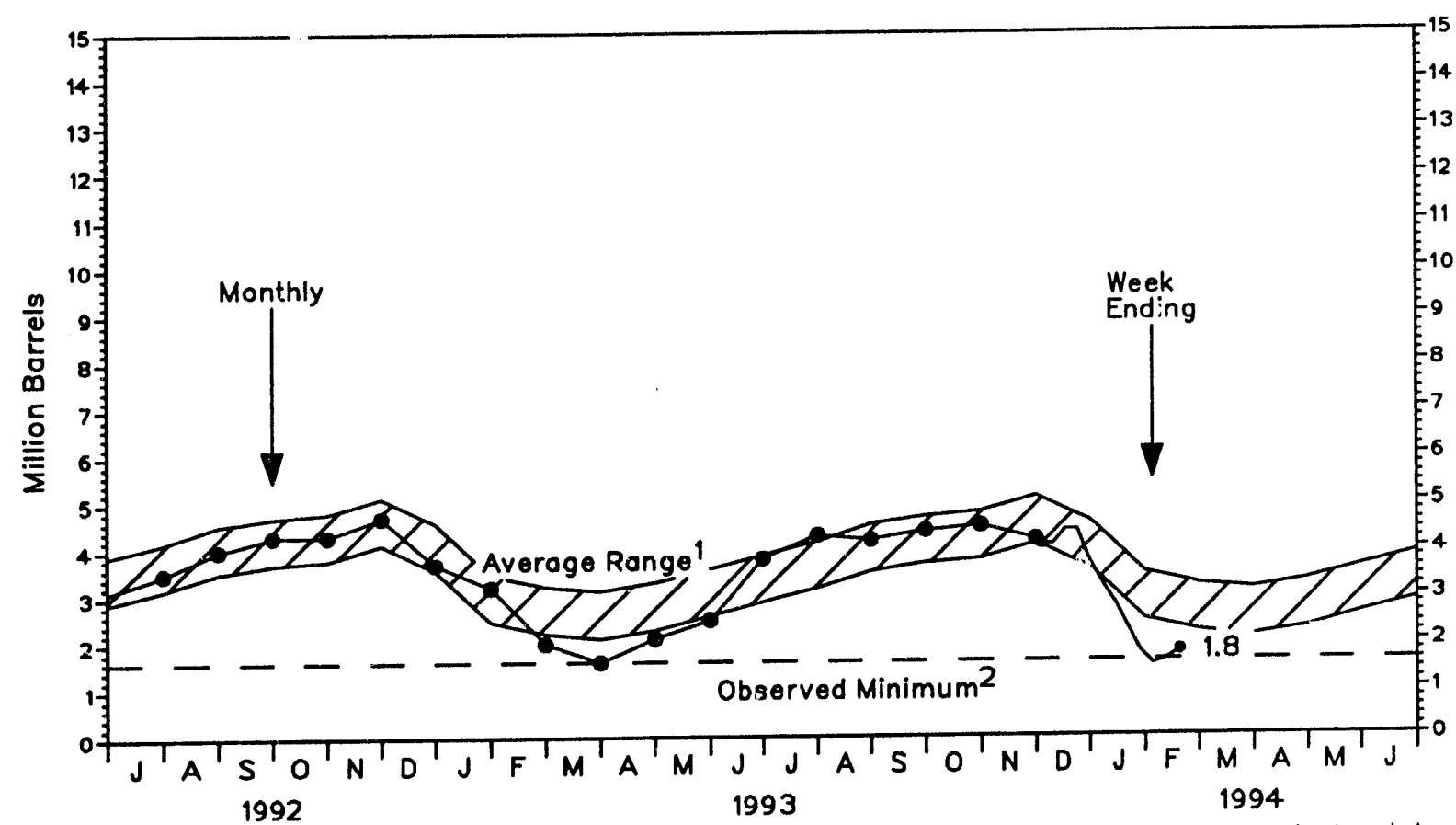

1 Average level and width of average range are based on 3 years of monthly data: July 1990-June 1993. The seasonal pattern is based on 7 years of monthly data.

2 The Observed Minimum for propane stocks in the last 36 month period was 1.6 million barrels, occurring in March 1993.

Source: - Data for Ranges and Seasonal Patterns: 1985-1992, Energy Information Administration (EIA), Petroleum Supply Annual; 1993, ElA, Petroloum Supply Monthly. - Monthly Data: 1992. ElA, Petroleum Supply Annual; 1993. EIA, Petroleum Supply Monthly. - Week-Ending Stocks: Estimates based on data collected on Form ElA-807, "Propane Telephone Survey."

\section{Period Ending 02/18/94 Energy Information Administration/Winter Fuels Report}


Figure 13. PADD II (Midwest) Propane Stocks

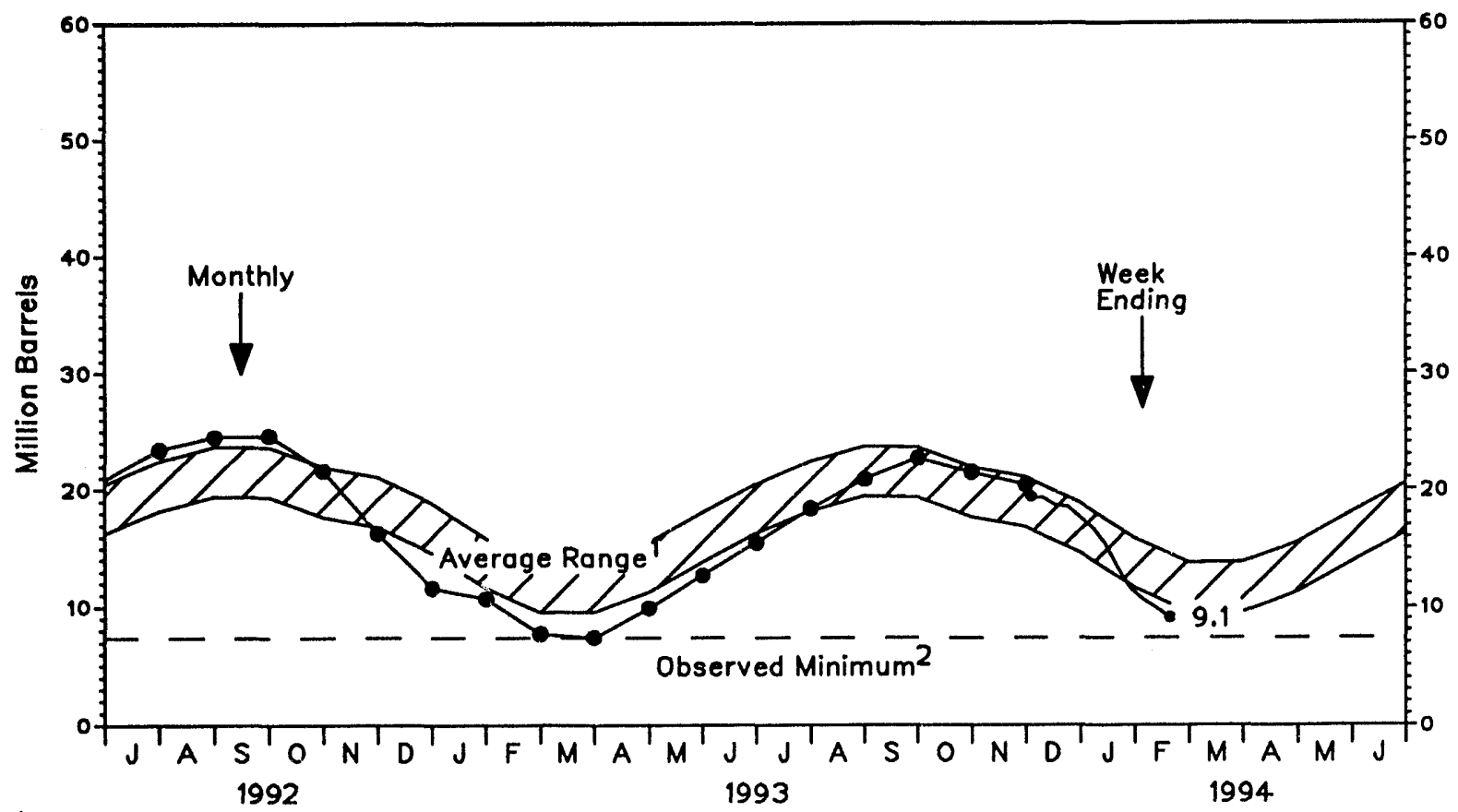

${ }^{1}$ Average level and width of average range are based on 3 years of monthly data: July 1990-June 1993. The seasonal pattern is based on 7 years of monthly data.

${ }_{2}$ The Observed Minimum for propane stocks in the last 36 month period was 7.4 million barrels, occurring in March 1993.

Source: - Data for Ranges and Seasonal Patterns: 1985-1992, Energy Information Administration (ElA), Petroleum Supply Annual; 1993, ElA, Petroleum Supply Monthly. • Monthly Data: 1992, ElA, Petroleum Supply Annual; 1993, ElA, Petroleum Supply Monthly. • Week-Ending Stocks: Estimates based on data collected on Form EIA-807, "Propane Telephone Survey."

Figure 14. PADD III (Gulf Coast) Propane Stocks

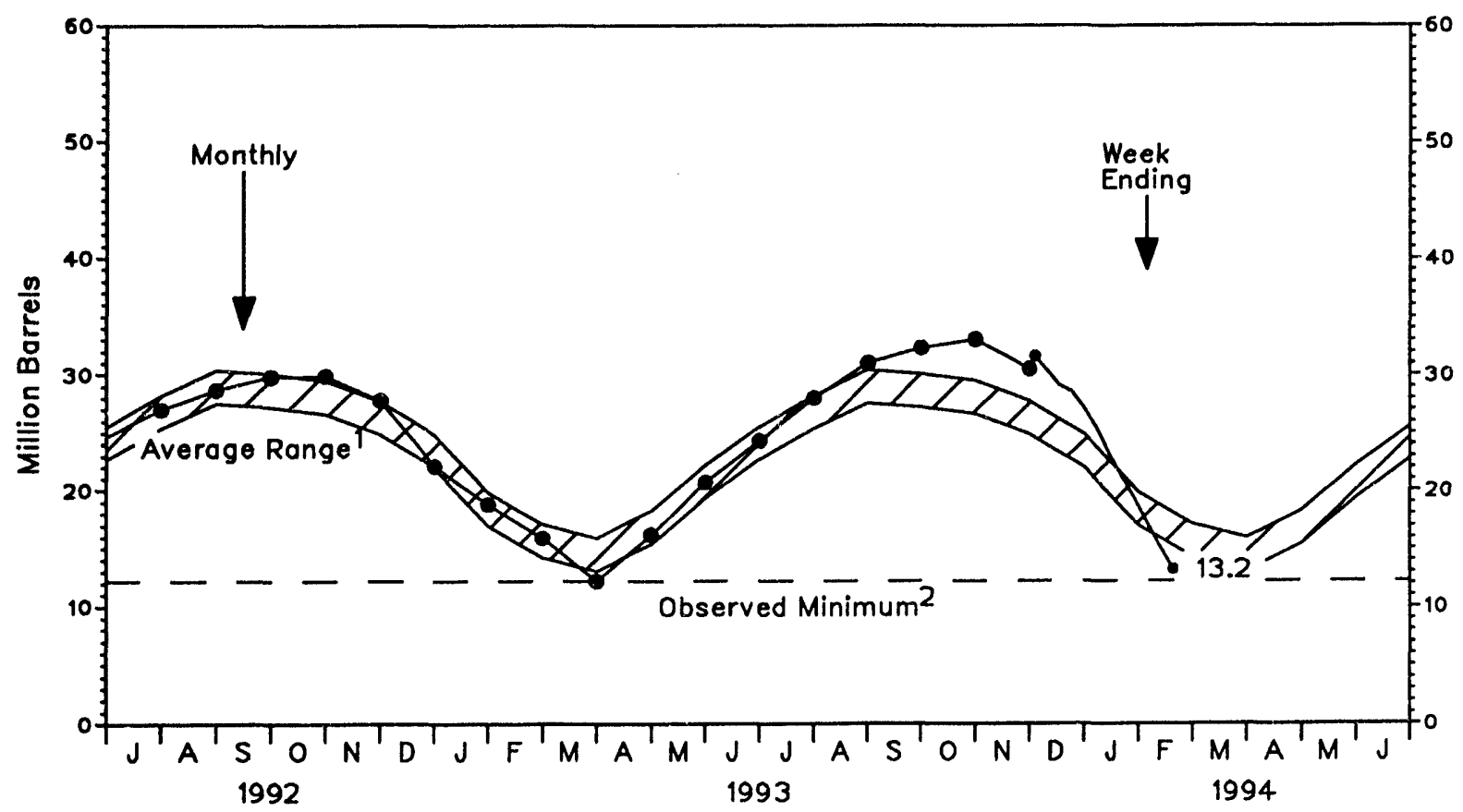

${ }^{1}$ Average level and width of average range are based on 3 years of monthly data: July 1990-June 1993. The seasonal pattern is based on 7 years of monthly data.

2 The Observed Minimum for propane stocks in the last 36 month period was 12.2 million barrels, occurring in March 1993.

Source: - Data for Ranges and Seasonal Patterns: 1985-1992, Energy Information Administration (EIA), Petroleum Supply Annual; 1993, ElA, Petroleum Supply Monthly. - Monthly Data: 1992, ElA, Petroleum Supply Annual; 1993, ElA, Petroleum Supply Monthly. - Week-Ending Stocks: Estimates based on data collected on Form EIA-807, "Propane Telephone Survey." 


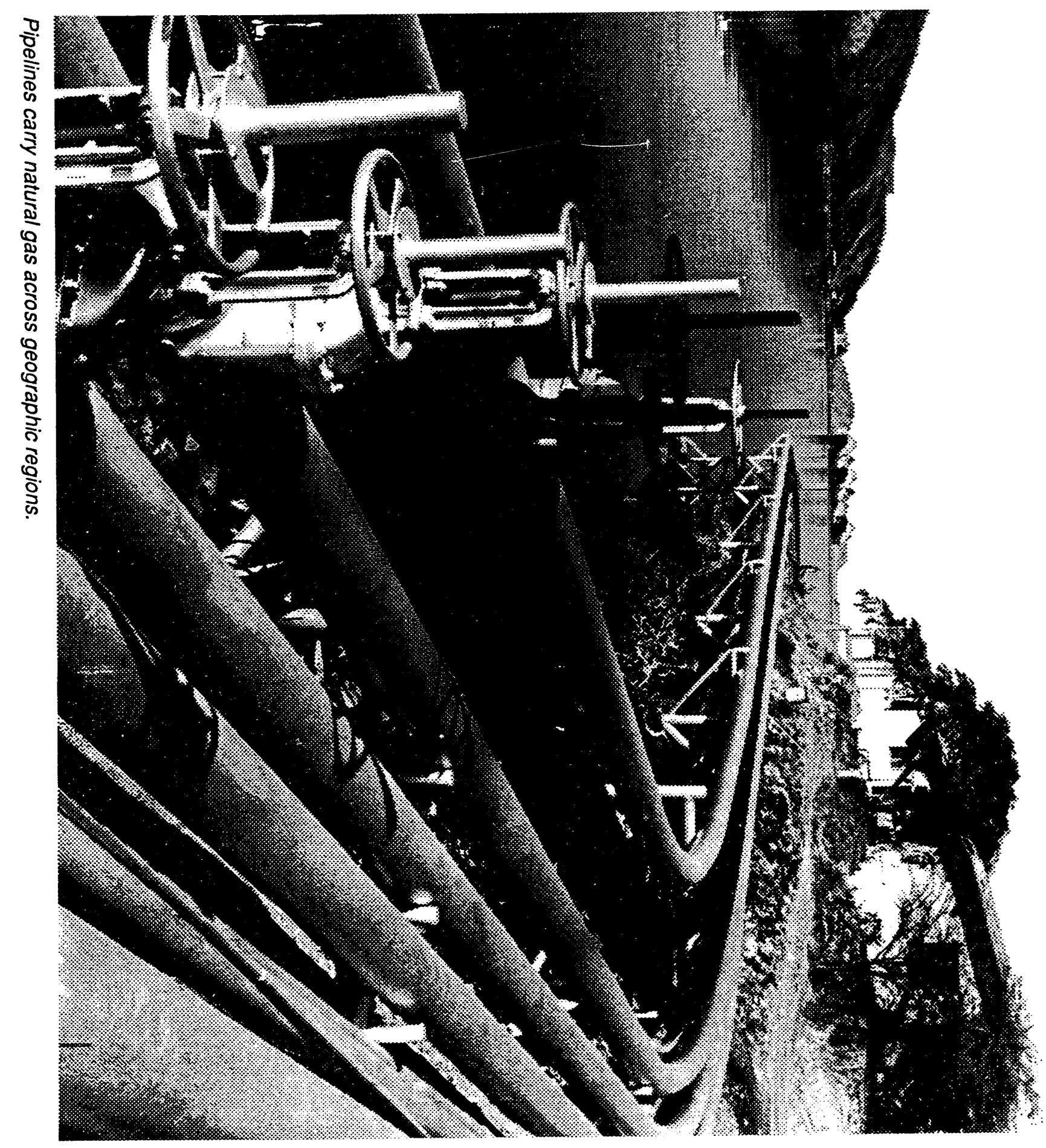

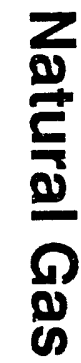


Table 3. Supply and Disposition of Dry Natural Gas in the United States

(Billion Cubic Feet)

\begin{tabular}{|c|c|c|c|c|c|c|c|c|c|}
\hline \multirow{2}{*}{$\begin{array}{l}\text { Year } \\
\text { and } \\
\text { Month }\end{array}$} & \multicolumn{5}{|c|}{ Supply } & \multirow{2}{*}{$\begin{array}{c}\text { Total } \\
\text { Supply/ } \\
\text { Dispositionc }\end{array}$} & \multicolumn{3}{|c|}{ Disposition } \\
\hline & \begin{tabular}{|c|} 
Total Dry \\
Gas \\
Production
\end{tabular} & $\begin{array}{c}\text { Withdrawals } \\
\text { from } \\
\text { Storage }\end{array}$ & $\begin{array}{c}\text { Supplemental } \\
\text { Gaseous } \\
\text { Fuels }\end{array}$ & Imports & $\begin{array}{c}\text { Balancing } \\
\text { Item }^{b}\end{array}$ & & $\begin{array}{c}\text { Additions } \\
\text { to } \\
\text { Storage }\end{array}$ & Exports & Consumptiond \\
\hline 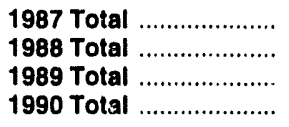 & $\begin{array}{l}16,621 \\
17,103 \\
17,311 \\
17,810\end{array}$ & $\begin{array}{l}1,905 \\
2,270 \\
2,854 \\
1,986\end{array}$ & $\begin{array}{l}101 \\
101 \\
107 \\
123\end{array}$ & $\begin{array}{r}993 \\
1,294 \\
1,382 \\
1,532\end{array}$ & $\begin{array}{l}-444 \\
-453 \\
-218 \\
-149\end{array}$ & $\begin{array}{l}19,176 \\
20,315 \\
21,435 \\
21,302\end{array}$ & $\begin{array}{l}1,911 \\
2,211 \\
2,528 \\
2,499\end{array}$ & $\begin{array}{r}54 \\
74 \\
107 \\
86\end{array}$ & $\begin{array}{l}17,211 \\
18,030 \\
18,801 \\
18,716\end{array}$ \\
\hline $\begin{array}{l}1991 \\
\begin{array}{l}19 \\
\text { January }\end{array} \\
\text { February } \\
\text { March }\end{array}$ & $\begin{array}{l}1,610 \\
1,417 \\
1,535 \\
1,462 \\
1,453 \\
1,385 \\
1,399 \\
1,405 \\
1,398 \\
1,509 \\
1,528 \\
1,597\end{array}$ & $\begin{array}{r}682 \\
409 \\
297 \\
104 \\
58 \\
42 \\
75 \\
82 \\
78 \\
103 \\
360 \\
461\end{array}$ & $\begin{array}{r}12 \\
10 \\
11 \\
9 \\
9 \\
8 \\
9 \\
9 \\
8 \\
10 \\
9 \\
11\end{array}$ & $\begin{array}{l}163 \\
138 \\
151 \\
144 \\
141 \\
133 \\
135 \\
127 \\
134 \\
157 \\
169 \\
181\end{array}$ & $\begin{array}{r}-44 \\
62 \\
-15 \\
65 \\
13 \\
-37 \\
-28 \\
-48 \\
-72 \\
-88 \\
-209 \\
-98\end{array}$ & $\begin{array}{l}2,423 \\
2,035 \\
1,979 \\
1,785 \\
1,675 \\
1,531 \\
1,590 \\
1,574 \\
1,545 \\
1,691 \\
1,856 \\
2,151\end{array}$ & $\begin{array}{l}115 \\
112 \\
129 \\
234 \\
331 \\
326 \\
299 \\
290 \\
304 \\
258 \\
150 \\
125\end{array}$ & $\begin{array}{r}10 \\
11 \\
10 \\
9 \\
8 \\
7 \\
8 \\
10 \\
11 \\
14 \\
15 \\
18\end{array}$ & $\begin{array}{l}2,299 \\
1,912 \\
1,840 \\
1,542 \\
1,337 \\
1,199 \\
1,283 \\
1,274 \\
1,231 \\
1,419 \\
1,691 \\
2,009\end{array}$ \\
\hline 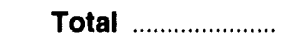 & 17,698 & 2,752 & 113 & 1.773 & .500 & 21,836 & 2,672 & 129 & 19,035 \\
\hline 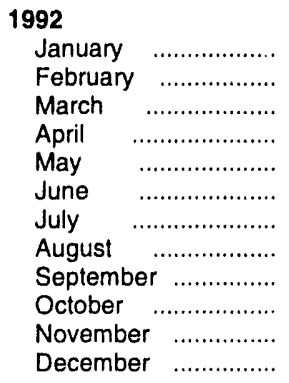 & $\begin{array}{l}1,586 \\
1,398 \\
1,475 \\
1,447 \\
1,485 \\
1,444 \\
1,491 \\
1,451 \\
1,437 \\
1,533 \\
1,514 \\
1,579\end{array}$ & $\begin{array}{r}624 \\
463 \\
397 \\
142 \\
44 \\
35 \\
42 \\
46 \\
40 \\
70 \\
282 \\
587\end{array}$ & $\begin{array}{r}12 \\
11 \\
11 \\
10 \\
9 \\
8 \\
8 \\
8 \\
8 \\
10 \\
11 \\
12\end{array}$ & $\begin{array}{l}165 \\
175 \\
180 \\
176 \\
174 \\
162 \\
167 \\
175 \\
166 \\
176 \\
210 \\
209\end{array}$ & $\begin{array}{r}-71 \\
42 \\
-42 \\
89 \\
68 \\
16 \\
-8 \\
-19 \\
-24 \\
-130 \\
-239 \\
-191\end{array}$ & $\begin{array}{l}2,315 \\
2,089 \\
2,022 \\
1,864 \\
1,780 \\
1,666 \\
1,700 \\
1,662 \\
1,629 \\
1,659 \\
1,778 \\
2,195\end{array}$ & $\begin{array}{r}60 \\
45 \\
74 \\
161 \\
344 \\
384 \\
373 \\
380 \\
362 \\
271 \\
88 \\
58\end{array}$ & $\begin{array}{l}16 \\
14 \\
23 \\
18 \\
19 \\
18 \\
16 \\
18 \\
18 \\
19 \\
19 \\
19\end{array}$ & $\begin{array}{l}2,239 \\
2,031 \\
1,926 \\
1,685 \\
1,418 \\
1,264 \\
1,311 \\
1,264 \\
1,249 \\
1,368 \\
1,672 \\
2,119\end{array}$ \\
\hline Total ...................... & 17,840 & 2,772 & 118 & 2,138 & -508 & 22,360 & 2,599 & 216 & 19,544 \\
\hline $\begin{array}{l}1993 \\
\text { January } \\
\text { February } \\
\text { March }\end{array}$ & $\begin{array}{r}1,608 \\
1,435 \\
1,579 \\
1,520 \\
1,545 \\
1,484 \\
1,527 \\
1,525 \\
1,502 \\
1,575 \\
\mathbf{E}_{1,543} \\
\mathbf{E}_{1,607}\end{array}$ & $\begin{array}{r}607 \\
578 \\
382 \\
110 \\
25 \\
44 \\
49 \\
100 \\
28 \\
102 \\
316 \\
499\end{array}$ & $\begin{array}{r}13 \\
12 \\
12 \\
10 \\
8 \\
10 \\
9 \\
9 \\
9 \\
10 \\
\mathrm{E}_{12} \\
\mathrm{E}_{13}\end{array}$ & $\begin{array}{r}198 \\
183 \\
199 \\
185 \\
160 \\
178 \\
190 \\
184 \\
188 \\
E_{189} \\
E_{182} \\
E_{198}\end{array}$ & $\begin{array}{r}-65 \\
3 \\
60 \\
78 \\
21 \\
-8 \\
-19 \\
-42 \\
2 \\
-155 \\
-206 \\
-110\end{array}$ & $\begin{array}{l}2,361 \\
2,212 \\
2,233 \\
1,904 \\
1,760 \\
1,708 \\
1,756 \\
1,776 \\
1,729 \\
1,721 \\
1,846 \\
2,207\end{array}$ & $\begin{array}{r}50 \\
27 \\
79 \\
222 \\
457 \\
418 \\
399 \\
421 \\
382 \\
255 \\
112 \\
58\end{array}$ & $\begin{array}{l}18 \\
13 \\
17 \\
12 \\
12 \\
11 \\
13 \\
10 \\
10 \\
E_{8} \\
E_{10} \\
E_{11}\end{array}$ & $\begin{array}{r}2,293 \\
2,172 \\
2,137 \\
1,671 \\
1,290 \\
1,280 \\
1,345 \\
1,345 \\
1,337 \\
1,458 \\
1,725 \\
E_{2,138}\end{array}$ \\
\hline Yotal ........................ & 18,452 & 2,840 & 128 & 2,235 & -440 & 23,214 & 2,879 & 142 & 20,192 \\
\hline
\end{tabular}

a Monthly and annual data for 1987 through 1992 include underground storage and liquefied natural gas storage. Data for January 1993 forward include underground storage only. See Appendix A, Explanatory Note 7 of Natural Gas Monthly (NGM) for discussion of computation procedures.

$b$ Represents quantities lost and imbalances in data due to differences among data sources. See Appendix A, Explanatory Note 10 of the NGM for ful discussion.

c Total data for 1987 through 1992 do not equal equivalent data in Table 1 of the Natural Gas Annual (NGA) 1992 due to the exclusion of intransit receipts and deliveries in the NGM.

Consists of pipeline fuel use, lease and plant fuel use, and deliveries to consuming sectors as shown in Table 3 of the NGM.

E = Estimated data.

Notes: - Data for 1987 through 1992 are final. All other data are preliminary unless otherwise indicated. $\bullet$ Geographic coverage is the 50 States and the District of Columbia. - Totals may not equal sum of components due to independent rounding.

Sources: - Total Dry Gas Production: EIA, Natural Gas Annual, 1987 through 1992; IOGCC, MMS reporting, and EIA estimates, January 1993 through current month. See Appendix A, Explanatory Note 3 of the Natural Gas Monthly for estimation procedures and revision policy. - Withdrawals from and Additions to Storage: EIA, Natural Gas Annual, 1987 through 1992; Form EIA-191, January 1993 through current month. • Supplemental Gaseous Fuels: ElA, Natural Gas Annual, 1987 through 1992; and ElA computations, January 1993 through current month. See Appendix A, Explanatory Note 2 of the Natural Gas Monthly for discussion of procedures and revision policy. - Imports and Exports: Form FPC-14, 1987 through 1992 ; and estimates, January 1993 through the current month. See Appendix A, Explanatory Note 4 of the Natural Gas Monthly for discussion of procedures and revision policy.

Consumption and Balancing ltem: EIA, Natural Gas Annual, 1987 through 1992; and EIA computations, January 1993 through current month. See Appendix A, Explanatory Notes 5 and 10 of the Natural Gas Monthly for discussion of computation procedures and revision policy. 
Table 4. Underground Natural Gas Storage In the United States (All Operators) (Billion Cubic Feet)

\begin{tabular}{|c|c|c|c|c|c|c|c|c|}
\hline \multirow{2}{*}{$\begin{array}{l}\text { Year } \\
\text { and } \\
\text { Month }\end{array}$} & \multicolumn{3}{|c|}{$\begin{array}{l}\text { Natural Gas in } \\
\text { Underground Storage } \\
\text { at End of Perlod }\end{array}$} & \multicolumn{2}{|c|}{$\begin{array}{c}\text { Change In Working Gas } \\
\text { from Same Period } \\
\text { Previous Year }\end{array}$} & \multicolumn{3}{|c|}{ Storage Activity } \\
\hline & $\begin{array}{l}\text { Base } \\
\text { Gas }\end{array}$ & $\begin{array}{c}\text { Working } \\
\text { Gas }\end{array}$ & Totalb & Volume & Percent & Injections & Whthdrawals & Netc \\
\hline $\begin{array}{l}1988 \text { Total } \ldots . . . . . \\
1989 \text { Total } \ldots . . . . . . \\
1990 \text { Total } \ldots . . . . . \\
1991 \text { Total" ......... }\end{array}$ & $\begin{array}{l}3,800 \\
3,812 \\
3,868 \\
3,954\end{array}$ & $\begin{array}{l}2,850 \\
2,513 \\
3,068 \\
2,824\end{array}$ & $\begin{array}{l}6,650 \\
6,325 \\
6,936 \\
6,778\end{array}$ & $\begin{array}{r}94 \\
-337 \\
555 \\
-244\end{array}$ & $\begin{array}{r}3.4 \\
-11.8 \\
22.1 \\
-8.0\end{array}$ & $\begin{array}{l}2,174 \\
2,491 \\
2,433 \\
2,608\end{array}$ & $\begin{array}{l}2,244 \\
2,804 \\
1,934 \\
2,689\end{array}$ & $\begin{array}{r}-69 \\
-313 \\
499 \\
-80\end{array}$ \\
\hline $\begin{array}{c}1992 \\
\text { January ............. } \\
\text { February .......... } \\
\text { March ............... } \\
\text { April ................ } \\
\text { May ............... } \\
\text { June ............... } \\
\text { July ............... } \\
\text { August ........... } \\
\text { September ....... } \\
\text { October ........... } \\
\text { November ........ } \\
\text { December ........ }\end{array}$ & $\begin{array}{l}4,061 \\
4,057 \\
4,046 \\
4,038 \\
4,044 \\
4,050 \\
4,064 \\
4,062 \\
4,061 \\
4,065 \\
4,061 \\
4,044\end{array}$ & $\begin{array}{l}2,216 \\
1,837 \\
1,545 \\
1,573 \\
1,848 \\
2,153 \\
2,460 \\
2,761 \\
3,044 \\
3,223 \\
3,054 \\
2,597\end{array}$ & $\begin{array}{l}6,277 \\
5,894 \\
5,591 \\
5,611 \\
5,892 \\
6,203 \\
6,524 \\
6,823 \\
7,105 \\
7,288 \\
7,115 \\
6,641\end{array}$ & $\begin{array}{l}-146 \\
-226 \\
-367 \\
-463 \\
-425 \\
-400 \\
-311 \\
-217 \\
-157 \\
-146 \\
-94 \\
-227\end{array}$ & $\begin{array}{r}-6.2 \\
-10.9 \\
-19.2 \\
-22.8 \\
-18.7 \\
-15.7 \\
-11.2 \\
-7.3 \\
-4.9 \\
-4.3 \\
-3.0 \\
-8.0\end{array}$ & $\begin{array}{r}68 \\
52 \\
81 \\
167 \\
330 \\
366 \\
357 \\
364 \\
346 \\
264 \\
95 \\
65\end{array}$ & $\begin{array}{r}591 \\
441 \\
381 \\
150 \\
53 \\
43 \\
50 \\
54 \\
48 \\
78 \\
276 \\
557\end{array}$ & $\begin{array}{r}-524 \\
-389 \\
-301 \\
18 \\
277 \\
323 \\
307 \\
309 \\
298 \\
186 \\
-181 \\
-491\end{array}$ \\
\hline Total ................... & - & - & - & - & - & 2,555 & 2,724 & .168 \\
\hline $\begin{array}{c}1993 \\
\text { January ............ } \\
\text { February .......... } \\
\text { March ............... } \\
\text { April ................ } \\
\text { May ................. } \\
\text { June ............... } \\
\text { July ................ } \\
\text { August ........... } \\
\text { September ....... } \\
\text { October ........... } \\
\text { November ........ } \\
\text { December ........ }\end{array}$ & $\begin{array}{l}4,041 \\
4,015 \\
3,994 \\
4,004 \\
4,026 \\
4,039 \\
4,039 \\
4,263 \\
4,255 \\
4,314 \\
4,325 \\
4,325\end{array}$ & $\begin{array}{l}2,046 \\
1,519 \\
1,238 \\
1,337 \\
1,744 \\
2,118 \\
2,471 \\
2,572 \\
2,904 \\
2,998 \\
2,781 \\
2,341\end{array}$ & $\begin{array}{l}6,087 \\
5,534 \\
5,232 \\
5,340 \\
5,771 \\
6,157 \\
6,510 \\
6,835 \\
7,159 \\
7,312 \\
7,106 \\
6,666\end{array}$ & $\begin{array}{r}-170 \\
-318 \\
-306 \\
-236 \\
-104 \\
-35 \\
11 \\
-189 \\
-140 \\
-225 \\
-273 \\
-256\end{array}$ & $\begin{array}{r}-7.7 \\
-17.3 \\
-19.8 \\
-15.0 \\
-5.6 \\
-1.6 \\
0.5 \\
-6.8 \\
-4.6 \\
-7.0 \\
-8.9 \\
-9.9\end{array}$ & $\begin{array}{r}50 \\
27 \\
79 \\
222 \\
457 \\
418 \\
399 \\
421 \\
382 \\
255 \\
112 \\
58\end{array}$ & $\begin{array}{r}607 \\
578 \\
382 \\
110 \\
25 \\
44 \\
49 \\
100 \\
28 \\
102 \\
316 \\
499\end{array}$ & $\begin{array}{r}-556 \\
-551 \\
-303 \\
112 \\
432 \\
374 \\
350 \\
321 \\
354 \\
154 \\
-204 \\
-441\end{array}$ \\
\hline Total ................... & - & - & - & - & - & 2,879 & 2,840 & 39 \\
\hline $\begin{array}{l}1994 \\
\quad \text { January .............. }\end{array}$ & $\varepsilon 4,325$ & $E_{1,591}$ & ${ }^{E} 5,916$ & Е.455 & E -22.2 & E 72 & E 822 & E. 750 \\
\hline
\end{tabular}

- Total as of December 31.

b Total underground storage capacity at the end of each calendar year (in billion cubic feet): 1987, 1988, and 1989 - 8,124; 1990 - 8,125; 1991 - 7,993; and 1992 7,932 .

c Positive rumbers indicate the volume of injections in excess of withdrawals. Negative numbers indicate the volume of withdrawals in excess of injections.

$\varepsilon=$ Estimateu data.

Notes: - Data for 1987 through 1992 are final. All other data are preliminary unless otherwise noted. See Appendix A, Explanatory Note 7 of the Natural Gas Monthly for discussion of revision policy. - Gas in storage at the end of a reporting period may not equal the quantity derived by adding or subtracting net injections or withdrawals during the period to the quantity of gas in storage at the beginning of the period. This is due to changes in the quantities of native gas included in base gas and/or losses in base gas due to migration from storage reservoirs. - Totals may not equal sum of components because of independent rounding. $\bullet$ Geographic coverage is the 50 States and the District of Columbia.

Source : Form EIA-191, Form FERC-8, and Form EIA-176. 
Figure 15. Underground Natural Gas Storage in the United States, 1989 - 1993

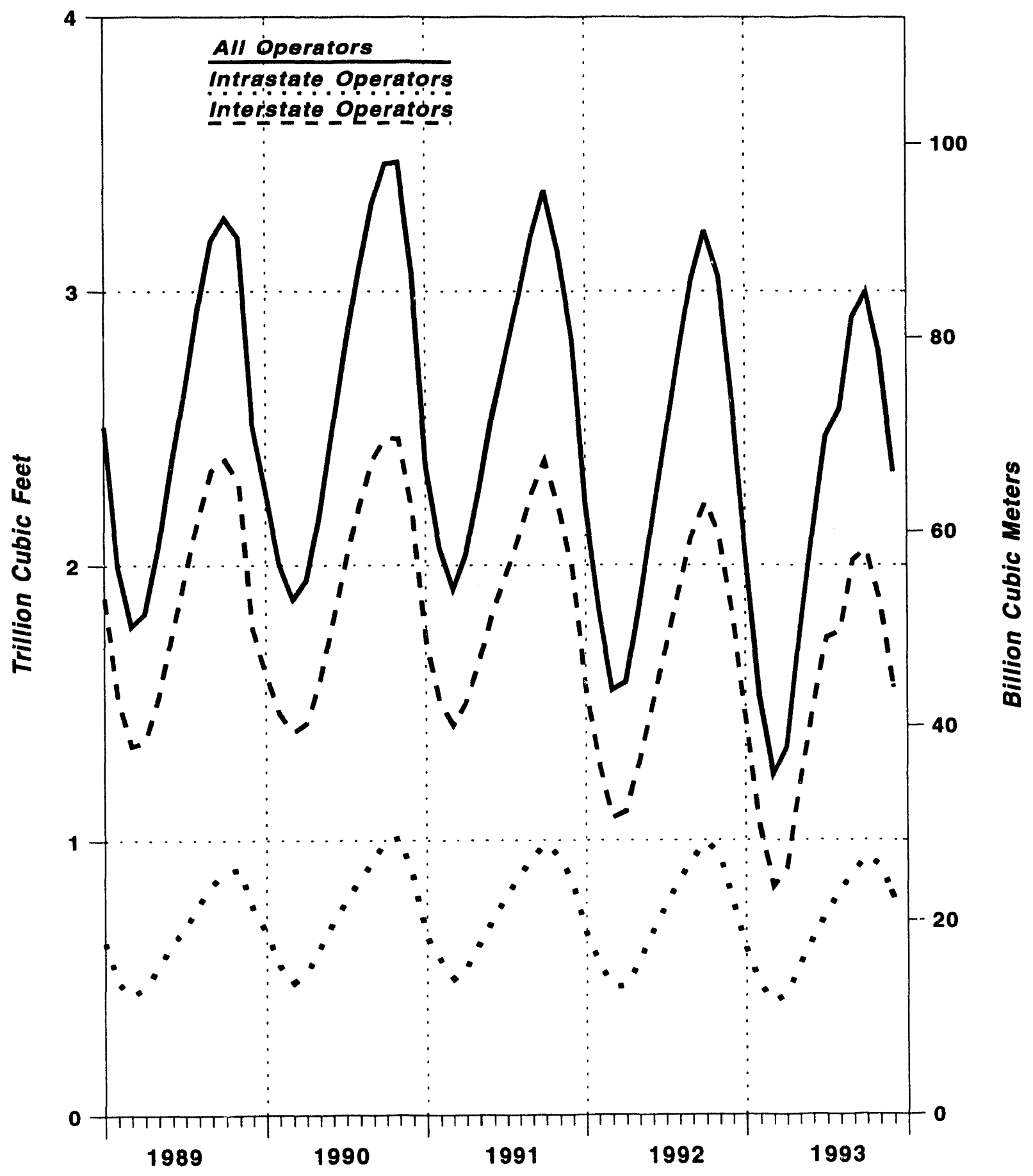

Sources: Energy Information Administration (EIA), Form EIA-191/FERC-8, "Underground Natural Gas Storage Report", and Form EIA-176, "Annual Report of Natural and Supplemental Gas Supply and Disposition", Natural Gas Annual, and Natural Gas Monthly. 
Table 5. Natural Gas Consumption by Petroleum Administration for Defense District (PADD) (Billion Cubic Feet)

\begin{tabular}{|c|c|c|c|c|c|c|c|c|}
\hline \multirow{2}{*}{$\begin{array}{c}\text { Year } \\
\text { and } \\
\text { Month }\end{array}$} & \multicolumn{4}{|c|}{ New England } & \multicolumn{4}{|c|}{ Central Atlantic } \\
\hline & Residential & Commercial & Industrial & $\begin{array}{l}\text { Electric } \\
\text { Utillities }\end{array}$ & Residentlal & Commercial & Industrial & $\begin{array}{l}\text { Electric } \\
\text { Utllities }\end{array}$ \\
\hline \multicolumn{9}{|l|}{1991} \\
\hline January $\quad$......... & 27 & 14 & 9 & 2 & 145 & 76 & 52 & 15 \\
\hline February ........ & 26 & 14 & 9 & 0 & 131 & 69 & 45 & 12 \\
\hline March $\quad$......... & 23 & 13 & 10 & 2 & 117 & 61 & 46 & 18 \\
\hline April $\quad$............. & 17 & 9 & 11 & 4 & 80 & 46 & 45 & 23 \\
\hline May $\quad$.......... & 10 & 6 & 12 & 4 & 44 & 28 & 40 & 33 \\
\hline June $\quad$........... & 6 & 4 & 11 & 5 & 26 & 22 & 37 & 35 \\
\hline July $\quad$............ & 5 & 4 & 8 & 8 & 23 & 22 & 36 & 44 \\
\hline August $\quad \ldots \ldots . .$. & 4 & 4 & 9 & 9 & 21 & 20 & 37 & 44 \\
\hline September ..... & 5 & 4 & 9 & 5 & 24 & 21 & 38 & 27 \\
\hline October $\quad \ldots . . . .$. & 8 & 5 & 11 & 5 & 43 & 29 & 44 & 22 \\
\hline November $\quad . . .$. & 14 & 8 & 11 & 2 & 78 & 44 & 46 & 19 \\
\hline Daceriber ..... & 21 & 12 & 11 & 0 & 118 & 66 & 48 & 16 \\
\hline Total $\quad \ldots \ldots \ldots . .$. & 166 & 97 & 122 & 47 & 850 & 504 & 514 & 306 \\
\hline \multicolumn{9}{|l|}{1992} \\
\hline January $\quad$......... & 29 & 15 & 12 & 0 & 150 & 77 & 56 & 11 \\
\hline February ....... & 30 & 16 & 14 & 0 & 148 & 77 & 57 & 15 \\
\hline March $\quad$.......... & 27 & 15 & 13 & 1 & 129 & 70 & 57 & 22 \\
\hline April $\quad$............ & 21 & 12 & 16 & 4 & 98 & 55 & 53 & 24 \\
\hline$\ldots \ldots \ldots$ & 13 & 8 & 14 & 4 & 55 & 32 & 48 & 24 \\
\hline ........... & 7 & 5 & 13 & 6 & 31 & 22 & 46 & 30 \\
\hline ............ & 5 & 5 & 12 & 8 & 25 & 21 & 47 & 42 \\
\hline August $\quad \ldots \ldots \ldots$ & 5 & 5 & 13 & 5 & 23 & 21 & 47 & 31 \\
\hline September ..... & 5 & 5 & 13 & 5 & 25 & 22 & 48 & 28 \\
\hline October $\quad . . . . . .$. & 9 & 7 & 13 & 4 & 50 & 32 & 52 & 16 \\
\hline November $\quad . . .$. & 16 & 10 & 14 & 4 & 82 & 46 & 58 & 14 \\
\hline December ..... & 24 & 13 & 14 & 0 & 128 & 69 & 59 & 13 \\
\hline ........... & 192 & 114 & 163 & 42 & 944 & 546 & 627 & 271 \\
\hline \multicolumn{9}{|l|}{1993} \\
\hline January $\quad . . . . . . .$. & 30 & 16 & 14 & 0 & 148 & 75 & 62 & 12 \\
\hline February ........ & 32 & 17 & 14 & 0 & 159 & 80 & 61 & 13 \\
\hline March $\quad \ldots \ldots \ldots$ & 29 & 16 & 14 & 3 & 151 & 77 & 64 & 16 \\
\hline April $\quad$............. & 20 & 11 & 13 & 4 & 93 & 51 & 56 & 16 \\
\hline ........... & 11 & 6 & 13 & 3 & 45 & 28 & 50 & 14 \\
\hline ........... & 7 & 5 & 13 & 3 & 32 & 24 & 50 & 26 \\
\hline ............. & 5 & 4 & 12 & 5 & 23 & 22 & 47 & 42 \\
\hline August $\quad \ldots \ldots \ldots$ & 5 & 4 & 16 & 5 & 22 & 21 & 48 & 33 \\
\hline September ..... & 5 & 4 & 15 & 3 & 25 & 22 & 48 & 21 \\
\hline October $\quad . . . . . .$. & 9 & 6 & 17 & 2 & 49 & 32 & 52 & 18 \\
\hline November ..... & 17 & 10 & 16 & 2 & 84 & 47 & 57 & 14 \\
\hline
\end{tabular}

See footnotes at end of table. 
Table 5. Natural Gas Consumption by Petroleum Administration for Defense District (PADD) (Continued) (Billion Cubic Feet)

\begin{tabular}{|c|c|c|c|c|c|c|c|c|}
\hline \multirow{2}{*}{$\begin{array}{c}\text { Year } \\
\text { and } \\
\text { Month }\end{array}$} & \multicolumn{4}{|c|}{ Lower Atlantlc } & \multicolumn{4}{|c|}{ PAD Dlstrict I } \\
\hline & Residential & Commercial & Industrlal & $\begin{array}{l}\text { Electric } \\
\text { Utllities }\end{array}$ & Residentlal & Commercial & Industrial & $\begin{array}{l}\text { Electrle } \\
\text { Utilities }\end{array}$ \\
\hline \multicolumn{9}{|l|}{1991} \\
\hline January ........ & 46 & 28 & 47 & 15 & 218 & 118 & 108 & 32 \\
\hline February ....... & 40 & 26 & 42 & 14 & 197 & 109 & 96 & 26 \\
\hline March & 33 & 23 & 45 & 16 & 173 & 97 & 101 & 36 \\
\hline April $\quad$............. & 18 & 16 & 43 & 17 & 115 & 71 & 99 & 44 \\
\hline ........... & 10 & 12 & 43 & 20 & 64 & 46 & 95 & 57 \\
\hline ............ & 8 & 11 & 41 & 21 & 40 & 37 & 89 & 61 \\
\hline July $\quad$............. & 7 & 10 & 41 & 26 & 35 & 36 & 85 & 78 \\
\hline August $\quad \ldots . . .$. & 7 & 11 & 43 & 26 & 32 & 35 & 89 & 79 \\
\hline September ..... & 7 & 11 & 43 & 21 & 36 & 36 & 90 & 53 \\
\hline October ........ & 12 & 13 & 45 & 19 & 63 & 47 & 100 & 46 \\
\hline November ..... & 28 & 19 & 44 & 15 & 120 & 71 & 101 & 36 \\
\hline December ..... & 39 & 25 & 44 & 14 & 178 & 103 & 103 & 30 \\
\hline$\ldots \ldots \ldots$ & 254 & 206 & 522 & 225 & 1,270 & 807 & 1,158 & 578 \\
\hline \multicolumn{9}{|l|}{1992} \\
\hline January ........ & 50 & 31 & 47 & 14 & 229 & 123 & 115 & 25 \\
\hline February ....... & 45 & 29 & 46 & 15 & 223 & 122 & 117 & 30 \\
\hline March $\quad . . . . . .$. & 34 & 24 & 51 & 19 & 190 & 109 & 121 & 42 \\
\hline April $\quad$............. & 25 & 20 & 47 & 20 & 144 & 87 & 116 & 48 \\
\hline ............ & 14 & 14 & 46 & 21 & 82 & 54 & 108 & 49 \\
\hline ............. & 9 & 12 & 44 & 23 & 47 & 39 & 103 & 59 \\
\hline .............. & 7 & 11 & 46 & 26 & 37 & 37 & 105 & 76 \\
\hline August $\quad . . . . . .$. & 7 & 11 & 45 & 22 & 35 & 37 & 105 & 58 \\
\hline September ..... & 7 & 11 & 45 & 22 & 37 & 38 & 106 & 55 \\
\hline October ......... & 14 & 14 & 44 & 13 & 73 & 53 & 109 & 33 \\
\hline November ..... & 28 & 19 & 47 & 13 & 126 & 75 & 119 & 31 \\
\hline December $\quad . . .$. & 44 & 29 & 47 & 11 & 196 & 111 & 120 & 24 \\
\hline$\ldots \ldots \ldots$ & 285 & 224 & 555 & 220 & 1,421 & 884 & 1,345 & 533 \\
\hline \multicolumn{9}{|l|}{1993} \\
\hline January ........ & 48 & 30 & 51 & 13 & 226 & 121 & 127 & 25 \\
\hline February ....... & 50 & 31 & 50 & 14 & 241 & 128 & 125 & 27 \\
\hline March $\quad . . . . . . .$. & 46 & 30 & 52 & 14 & 226 & 123 & 130 & 33 \\
\hline April $\quad \ldots \ldots \ldots \ldots$ & 28 & 20 & 49 & 14 & 141 & 82 & 118 & 34 \\
\hline ............ & 12 & 13 & 45 & 17 & 68 & 47 & 108 & 34 \\
\hline ........... & 8 & 11 & 49 & 21 & 47 & 40 & 112 & 50 \\
\hline ............ & 7 & 11 & 51 & 25 & 35 & 37 & 110 & 72 \\
\hline August $\quad \ldots . . .$. & 7 & 11 & 54 & 24 & 34 & 36 & 118 & 62 \\
\hline September ..... & 7 & 11 & 46 & 20 & 37 & 37 & 109 & 44 \\
\hline October $\quad . . . . . . .$. & 13 & 14 & 50 & 14 & 71 & 52 & 119 & 34 \\
\hline November $\quad . . .$. & 27 & 20 & 51 & 12 & 128 & 77 & 124 & 28 \\
\hline
\end{tabular}

See footnotes at end of table. 
Table 5. Natural Gas Consumption by Petroleum Administration for Defense District (PADD) (Continued) (Billion Cubic Feet)

\begin{tabular}{|c|c|c|c|c|c|c|c|c|}
\hline \multirow{2}{*}{$\begin{array}{c}\text { Year } \\
\text { and } \\
\text { Month }\end{array}$} & \multicolumn{4}{|c|}{ PAD District II } & \multicolumn{4}{|c|}{ PAD District III } \\
\hline & Residential & Commercial & Industrial & $\begin{array}{l}\text { Electric } \\
\text { Utilities }\end{array}$ & Residential & Commercial & Industrial & $\begin{array}{l}\text { Electric } \\
\text { Utilitles }\end{array}$ \\
\hline \multicolumn{9}{|l|}{1991} \\
\hline January & 385 & 189 & 203 & 16 & 84 & 45 & 262 & 87 \\
\hline February ....... & 292 & 152 & 178 & 13 & 64 & 36 & 230 & 68 \\
\hline March $\quad$.......... & 245 & 125 & 173 & 16 & 48 & 30 & 241 & 95 \\
\hline April $\quad$............... & 147 & 76 & 152 & 20 & 29 & 24 & 244 & 112 \\
\hline ............. & 87 & 49 & 142 & 27 & 18 & 18 & 252 & 132 \\
\hline ............ & 49 & 32 & 134 & 29 & 15 & 17 & 241 & 140 \\
\hline July $\quad$.............. & 43 & 37 & 136 & 39 & 14 & 18 & 265 & 168 \\
\hline August $\quad \ldots \ldots . .$. & 40 & 35 & 140 & 36 & 13 & 16 & 269 & 159 \\
\hline September ..... & 55 & 37 & 142 & 26 & 14 & 14 & 257 & 118 \\
\hline October $\quad$......... & 102 & 57 & 156 & 22 & 18 & 17 & 273 & 127 \\
\hline November $\quad . . .$. & 224 & 110 & 172 & 19 & 41 & 28 & 268 & 95 \\
\hline December ..... & 295 & 147 & 185 & 16 & 60 & 36 & 280 & 81 \\
\hline$\ldots \ldots \ldots$ & 1,964 & 1,047 & 1,913 & 279 & 419 & 299 & 3,081 & 1,382 \\
\hline \multicolumn{9}{|l|}{1992} \\
\hline January & 339 & 165 & 196 & 16 & 76 & 42 & 279 & 81 \\
\hline February $\quad . . . . .$. & 289 & 147 & 187 & 16 & 68 & 37 & 240 & 77 \\
\hline March $\quad$.......... & 251 & 123 & 186 & 20 & 44 & 28 & 275 & 96 \\
\hline April $\quad$............... & 184 & 97 & 172 & 20 & 33 & 25 & 267 & 109 \\
\hline ........... & 102 & 53 & 153 & 20 & 20 & 20 & 260 & 116 \\
\hline ............ & 61 & 35 & 142 & 20 & 16 & 17 & 245 & 139 \\
\hline July $\quad$............ & 47 & 34 & 139 & 25 & 15 & 20 & 259 & 168 \\
\hline August $\quad \ldots . . .$. & 46 & 34 & 139 & 22 & 14 & 19 & 249 & 138 \\
\hline September ..... & 53 & 35 & 144 & 21 & 14 & 17 & 245 & 130 \\
\hline October $\quad . . . . . .$. & 111 & 61 & 164 & 13 & 16 & 18 & 248 & 103 \\
\hline November $\quad . . .$. & 206 & 108 & 181 & 13 & 34 & 28 & 244 & 89 \\
\hline Decrmber $\quad . . .$. & 316 & 160 & 195 & 15 & 67 & 39 & 263 & 84 \\
\hline$\ldots \ldots \ldots$ & 2,003 & 1,052 & 1,998 & 220 & 417 & 310 & 3,074 & 1,330 \\
\hline \multicolumn{9}{|l|}{1993} \\
\hline January & 368 & 180 & 203 & 14 & 77 & 44 & 273 & 77 \\
\hline February $\quad . . . . .$. & 334 & 166 & 200 & 14 & 67 & 39 & 248 & 73 \\
\hline March $\quad$.......... & 311 & 155 & 200 & 15 & 59 & 35 & 270 & 95 \\
\hline April $\quad \ldots \ldots \ldots \ldots$ & 196 & 100 & 171 & 14 & 39 & 29 & 258 & 87 \\
\hline .......... & 92 & 49 & 154 & 14 & 22 & 22 & 237 & 94 \\
\hline$\ldots \ldots \ldots$ & 62 & 35 & 149 & 20 & 16 & 22 & 250 & 146 \\
\hline July $\quad$........... & 45 & 33 & 138 & 34 & 14 & 22 & 271 & 188 \\
\hline August $\quad \ldots \ldots . .$. & 41 & 32 & 146 & 40 & 13 & 21 & 259 & 197 \\
\hline September ..... & 56 & 37 & 147 & 18 & 13 & 18 & 323 & 144 \\
\hline October $\quad . . . . . .$. & 118 & 63 & 169 & 19 & 19 & 19 & 260 & 124 \\
\hline November $\quad . . .$. & 209 & 109 & 181 & 17 & 42 & 29 & 239 & 105 \\
\hline
\end{tabular}

See footnotes at end of table. 
Table 5. Natural Gas Consumption by Petroleum Administration for Defense District (PADD) (Continued) (Billion Cubic Feet)

\begin{tabular}{|c|c|c|c|c|c|c|c|c|}
\hline \multirow{2}{*}{$\begin{array}{l}\text { Year } \\
\text { and } \\
\text { Month }\end{array}$} & \multicolumn{4}{|c|}{ PAD Dlstrict IV } & \multicolumn{4}{|c|}{ PAD District V } \\
\hline & Residential & Commercial & Industrial & $\begin{array}{l}\text { Electrlc } \\
\text { Utilities }\end{array}$ & Residential & Commercial & Industrial & $\begin{array}{l}\text { Electric } \\
\text { Utilities }\end{array}$ \\
\hline \multicolumn{9}{|l|}{1991} \\
\hline January $\quad$........ & 49 & 29 & 23 & 1 & 108 & 51 & 76 & 36 \\
\hline February & 38 & 23 & 20 & 1 & 72 & 39 & 66 & 38 \\
\hline March $\quad$.......... & 30 & 18 & 21 & 1 & 77 & 40 & 71 & 46 \\
\hline ............ & 22 & 13 & 19 & 1 & 60 & 41 & 73 & 38 \\
\hline ........... & 16 & 10 & 18 & 1 & 44 & 31 & 65 & 32 \\
\hline ............ & 9 & 6 & 17 & 1 & 35 & 28 & 65 & 29 \\
\hline .............. & 6 & 4 & 17 & 2 & 29 & 29 & 69 & 44 \\
\hline August $\quad \ldots \ldots .$. & 6 & 4 & 17 & 2 & 26 & 23 & 71 & 53 \\
\hline September ..... & 6 & 5 & 19 & 1 & 27 & 29 & 74 & 64 \\
\hline October $\quad . . . . . . .$. & 11 & 7 & 21 & 2 & 31 & 34 & 75 & 68 \\
\hline November $\ldots . .$. & 25 & 15 & 23 & 2 & 50 & 31 & 64 & 47 \\
\hline December $\quad . . .$. & 39 & 22 & 25 & 2 & 86 & 43 & 71 & 42 \\
\hline Total & 257 & 157 & 240 & 15 & 646 & 419 & 839 & 536 \\
\hline \multicolumn{9}{|l|}{1992} \\
\hline January $\quad$........ & 41 & 24 & 25 & 1 & 100 & 56 & 87 & 46 \\
\hline February $\quad . . . . .$. & 37 & 22 & 23 & 1 & 80 & 39 & 77 & 46 \\
\hline March $\quad$......... & 28 & 16 & 23 & 1 & 62 & 37 & 70 & 48 \\
\hline April $\quad \ldots \ldots \ldots \ldots$ & 21 & 13 & 21 & 1 & 48 & 29 & 52 & 51 \\
\hline ........... & 12 & 7 & 20 & 1 & 35 & 35 & 80 & 50 \\
\hline ........... & 9 & 6 & 20 & 1 & 29 & 29 & 67 & 46 \\
\hline .............. & 7 & 5 & 21 & 1 & 26 & 27 & 64 & 62 \\
\hline August $\quad \ldots \ldots \ldots$ & 6 & 4 & 20 & 1 & 25 & 27 & 69 & 82 \\
\hline September ..... & 7 & 5 & 21 & 1 & 26 & 26 & 71 & 66 \\
\hline October $\quad . . . . . .$. & 11 & 8 & 23 & 1 & 31 & 27 & 63 & 62 \\
\hline November $\quad . . .$. & 23 & 15 & 26 & 1 & 48 & 31 & 72 & 56 \\
\hline December ..... & 41 & 25 & 27 & 1 & 98 & 46 & 71 & 52 \\
\hline$\ldots \ldots \ldots$ & 242 & 149 & 267 & 14 & 607 & 409 & 843 & 668 \\
\hline \multicolumn{9}{|l|}{1993} \\
\hline January ........ & 48 & 28 & 29 & 1 & 115 & 49 & 67 & 47 \\
\hline February ....... & 41 & 24 & 27 & 1 & 88 & 51 & 72 & 48 \\
\hline March $\quad \ldots . . . .$. & 37 & 22 & 25 & 1 & 70 & 39 & 73 & 49 \\
\hline April $\quad \ldots \ldots \ldots \ldots$ & 25 & 14 & 24 & 1 & 49 & 31 & 68 & 38 \\
\hline$\ldots \ldots \ldots$ & 15 & 9 & 24 & 1 & 38 & 29 & 69 & 24 \\
\hline ............ & 9 & 6 & 23 & 1 & 31 & 24 & 65 & 37 \\
\hline ............. & 7 & 5 & 22 & 2 & 28 & 26 & 76 & 38 \\
\hline August $\quad \ldots \ldots .$. & 6 & 5 & 22 & 2 & 27 & 22 & 69 & 56 \\
\hline September ..... & 8 & 6 & 24 & 1 & 28 & 25 & 73 & 51 \\
\hline October $\quad \ldots . . .$. & 13 & 8 & 24 & 1 & 31 & 29 & 81 & 54 \\
\hline November $\quad . . .$. & 26 & 16 & 26 & 1 & 51 & 33 & 74 & 57 \\
\hline
\end{tabular}

Notes: - Data for 1987 through 1992 are final. All other data are preliminary unless otherwise indicated. - Geographic coverage is the 50 States and the District of Columbia. - Totals may not equal sum of components because of independent rounding.

Sources: All data except electric utility: EIA, Natural Gas Annual, 1991 through 1992; and Form EIA-857 and computations January 1993 through the current month. See Appendix A, Explanatory Note 5 of the Natural Gas Monthly for computation procedures and revision policy. Electric utility data: Form EIA-759, "Monthly Power Plant Report" (formerly Form FPC-4). 
Figure 16. Natural Gas Deliveries to Consumers in the United States, 1989 - 1993

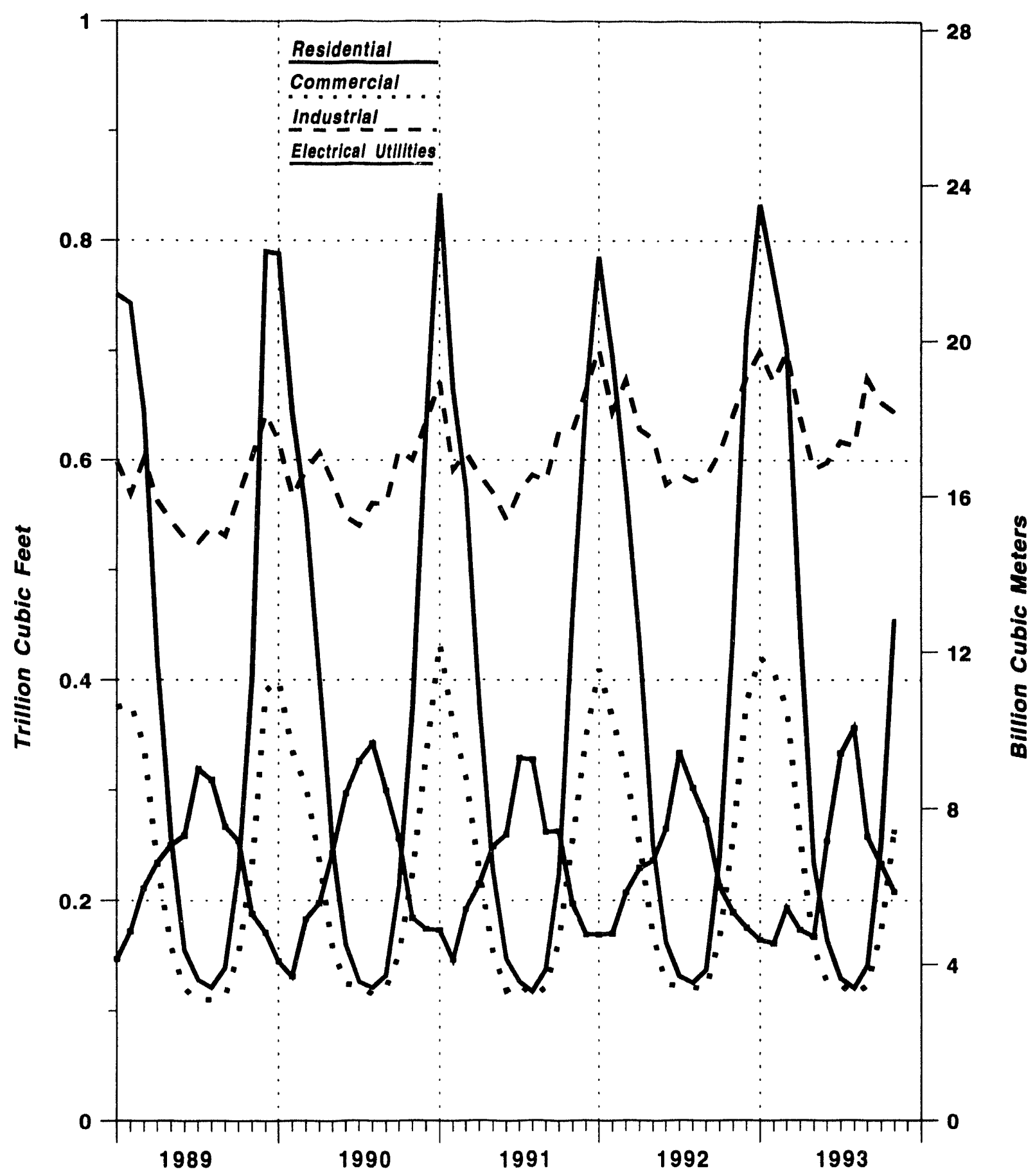

Sources: Energy Information Administration (EIA), Form ElA-857, "Monthly Report of Natural Gas Purchases and Deliveries to Consumers", Form EIA-759, "Monthly Power Plant Report", Natural Gas Annual and Natural Gas Monthly. 
Table 6. Selected National Average Natural Gas Prices in the United States (Dollars per Thousand Cubic Feet)

\begin{tabular}{|c|c|c|c|c|c|c|c|c|}
\hline \multirow{2}{*}{$\begin{array}{l}\text { Year } \\
\text { and } \\
\text { Month }\end{array}$} & \multirow{2}{*}{$\begin{array}{c}\text { Wellhead } \\
\text { Prlcea }\end{array}$} & \multicolumn{2}{|c|}{$\begin{array}{c}\text { Major Interstate } \\
\text { Pipeline Companies }\end{array}$} & \multirow{2}{*}{$\begin{array}{l}\text { City } \\
\text { Gate }\end{array}$} & \multicolumn{4}{|c|}{ Delivered to Consumers } \\
\hline & & Importsb & $\begin{array}{l}\text { Purchased } \\
\text { from } \\
\text { Producersb }\end{array}$ & & Residentlal & Commercial & Industrial & $\begin{array}{l}\text { Electric } \\
\text { Utilities }\end{array}$ \\
\hline 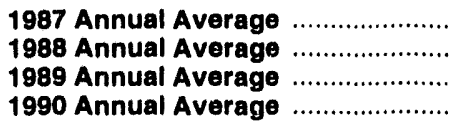 & $\begin{array}{l}1.67 \\
1.69 \\
1.69 \\
1.71\end{array}$ & $\begin{array}{l}2.17 \\
2.00 \\
2.04 \\
2.03\end{array}$ & $\begin{array}{l}2.10 \\
2.13 \\
2.18 \\
2.19\end{array}$ & $\begin{array}{l}2.87 \\
2.92 \\
3.01 \\
3.03\end{array}$ & $\begin{array}{l}5.54 \\
5.47 \\
5.64 \\
5.80\end{array}$ & $\begin{array}{l}4.77 \\
4.63 \\
4.74 \\
4.83\end{array}$ & $\begin{array}{l}2.94 \\
2.95 \\
2.96 \\
2.93\end{array}$ & $\begin{array}{l}2.32 \\
2.33 \\
2.43 \\
2.38\end{array}$ \\
\hline $\begin{array}{l}1991 \\
\text { January } \\
\text { February } \\
\text { March }\end{array}$ & $\begin{array}{l}1.96 \\
1.62 \\
1.49 \\
1.50 \\
1.48 \\
1.43 \\
1.34 \\
1.43 \\
1.59 \\
1.82 \\
1.89 \\
2.00\end{array}$ & $\begin{array}{l}2.20 \\
2.10 \\
1.92 \\
2.03 \\
1.99 \\
2.03 \\
2.11 \\
1.71 \\
1.84 \\
2.00 \\
2.20 \\
2.09\end{array}$ & $\begin{array}{l}2.19 \\
1.93 \\
2.02 \\
1.87 \\
1.96 \\
1.75 \\
1.79 \\
1.71 \\
1.76 \\
1.94 \\
2.02 \\
2.11\end{array}$ & $\begin{array}{l}3.08 \\
2.94 \\
2.78 \\
2.74 \\
2.76 \\
2.86 \\
2.74 \\
2.78 \\
2.91 \\
2.92 \\
2.92 \\
3.05\end{array}$ & $\begin{array}{l}5.54 \\
5.56 \\
5.60 \\
5.90 \\
6.28 \\
6.97 \\
7.23 \\
7.36 \\
6.92 \\
6.20 \\
5.51 \\
5.51\end{array}$ & $\begin{array}{l}4.94 \\
4.94 \\
4.89 \\
4.87 \\
4.65 \\
4.80 \\
4.50 \\
4.73 \\
4.57 \\
4.58 \\
4.71 \\
4.84\end{array}$ & $\begin{array}{l}3.25 \\
2.97 \\
2.75 \\
2.68 \\
2.40 \\
2.34 \\
2.23 \\
2.29 \\
2.40 \\
2.69 \\
2.84 \\
3.09\end{array}$ & $\begin{array}{l}2.70 \\
2.35 \\
2.21 \\
2.10 \\
2.01 \\
1.94 \\
1.88 \\
1.96 \\
2.19 \\
2.35 \\
2.43 \\
2.64\end{array}$ \\
\hline Annual Average $\ldots \ldots \ldots \ldots \ldots \ldots \ldots$ & 1.64 & 2.02 & 1.92 & 2.90 & 5.82 & 4.81 & 2.69 & 2.18 \\
\hline $\begin{array}{l}1992 \\
\text { January } \\
\text { February } \\
\text { March }\end{array}$ & $\begin{array}{l}1.74 \\
1.26 \\
1.35 \\
1.42 \\
1.51 \\
1.62 \\
1.55 \\
1.84 \\
1.92 \\
2.38 \\
2.13 \\
2.07\end{array}$ & $\begin{array}{l}2.20 \\
1.98 \\
1.45 \\
2.01 \\
1.79 \\
2.03 \\
1.89 \\
1.85 \\
2.05 \\
2.13 \\
2.32 \\
1.92\end{array}$ & $\begin{array}{l}2.10 \\
1.70 \\
1.90 \\
1.73 \\
1.99 \\
2.16 \\
1.86 \\
2.14 \\
2.13 \\
2.69 \\
2.33 \\
2.40\end{array}$ & $\begin{array}{l}2.90 \\
2.70 \\
2.61 \\
2.74 \\
2.90 \\
3.00 \\
3.01 \\
3.18 \\
3.23 \\
3.50 \\
3.33 \\
3.17\end{array}$ & $\begin{array}{l}5.53 \\
5.54 \\
5.50 \\
5.62 \\
6.15 \\
6.84 \\
7.27 \\
7.45 \\
7.15 \\
6.52 \\
6.02 \\
5.74\end{array}$ & $\begin{array}{l}4.85 \\
5.03 \\
4.77 \\
4.77 \\
4.59 \\
4.72 \\
4.64 \\
4.73 \\
4.69 \\
4.90 \\
5.12 \\
5.11\end{array}$ & $\begin{array}{l}3.04 \\
2.78 \\
2.58 \\
2.54 \\
2.44 \\
2.53 \\
2.54 \\
2.71 \\
2.82 \\
3.21 \\
3.26 \\
3.38\end{array}$ & $\begin{array}{l}2.49 \\
2.03 \\
1.99 \\
2.07 \\
2.11 \\
2.18 \\
2.13 \\
2.42 \\
2.51 \\
3.04 \\
2.87 \\
2.81\end{array}$ \\
\hline Annual Average $\ldots \ldots \ldots \ldots \ldots \ldots \ldots$ & 1.74 & 1.97 & 2.09 & 3.01 & 5.89 & 4.88 & 2.84 & 2.36 \\
\hline 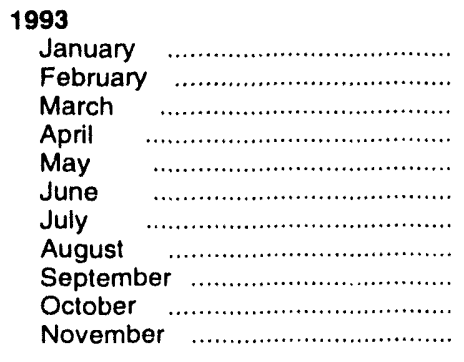 & $\begin{array}{r}1.96 \\
1.72 \\
1.89 \\
2.05 \\
2.30 \\
1.87 \\
1.91 \\
2.00 \\
2.11 \\
1.93 \\
E_{2} .06\end{array}$ & $\begin{array}{l}2.02 \\
1.91 \\
1.78 \\
2.15 \\
2.13 \\
1.95 \\
1.78 \\
2.02 \\
2.17 \\
1.97 \\
1.85\end{array}$ & $\begin{array}{l}2.17 \\
1.94 \\
2.20 \\
2.34 \\
2.81 \\
2.03 \\
2.02 \\
2.35 \\
2.58 \\
2.05 \\
2.32\end{array}$ & $\begin{array}{l}3.11 \\
2.94 \\
3.06 \\
3.24 \\
3.58 \\
3.44 \\
3.34 \\
3.35 \\
3.52 \\
3.15 \\
3.14\end{array}$ & $\begin{array}{l}5.71 \\
5.71 \\
5.66 \\
5.99 \\
6.72 \\
7.32 \\
7.83 \\
8.10 \\
7.74 \\
6.75 \\
6.16\end{array}$ & $\begin{array}{l}5.18 \\
5.08 \\
5.06 \\
5.13 \\
5.21 \\
5.31 \\
5.03 \\
5.26 \\
5.27 \\
5.12 \\
5.13\end{array}$ & $\begin{array}{l}3.26 \\
3.12 \\
3.08 \\
3.13 \\
3.24 \\
2.95 \\
2.71 \\
2.86 \\
3.03 \\
2.88 \\
3.09\end{array}$ & $\begin{array}{l}2.70 \\
2.55 \\
2.61 \\
2.75 \\
2.90 \\
2.47 \\
2.46 \\
2.60 \\
2.69 \\
2.45 \\
\text { NA }\end{array}$ \\
\hline 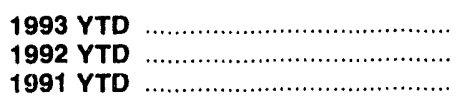 & $\begin{array}{l}1.98 \\
1.70 \\
1.60\end{array}$ & $\begin{array}{l}1.98 \\
1.97 \\
2.01\end{array}$ & $\begin{array}{l}2.25 \\
2.07 \\
1.90\end{array}$ & $\begin{array}{l}3.20 \\
2.98 \\
2.88\end{array}$ & $\begin{array}{l}6.16 \\
5.92 \\
5.88\end{array}$ & $\begin{array}{l}5.14 \\
4.84 \\
4.81\end{array}$ & $\begin{array}{l}3.04 \\
2.78 \\
2.65\end{array}$ & $\begin{array}{l}2.38 \\
2.34 \\
2.15\end{array}$ \\
\hline
\end{tabular}

- See Appendix A, Explantory Note 8 of the Natural Gas Monthly for discussion of wellhead price.

b See Appendix A. Explantory Note 9 of the Natural Gas Monthly for discussion of major interstate pipeline company data.

c See Table Notes and Sources for explanation of break in series for consumer prices in 1988.

E Estimated data

NA = Not Available.

Notes: - Data for 1987 through 1992 are final. All other data are preliminary unless otherwise indicated. - Geographic coverage is the 50 States and the District of Columbia. Price for gas deliverd to industrial consumers for 1987 through 1988 imputed average for volumes of gas delivered for the account of others. From 1988 on, prices reflect on-system sales prices only. The change in series in 1988 affects the commercial,

industrial sector prices.

Sources: - Average wellhead price: EIA, Natural Gas Annual, 1992, 1987 through 1992; and EIA estimates, January 1993 through current month. See Appendix A, Explanatory Note 8 of the Natural Gas Monthly for estimation procedures and revision policy. Imports and Interstate Pipeline Company Purchases: Form FERC-11. Average City Gate, Residential, Commercial and Industrial average prices for 1987 through current month from Form ElA-857. See Appendix A, Explanatory Note 5 of the Natural Gas Monthly for discussion of revision policy. - Electric Utitities averages from Form FERC-423, "Monthly Report of Cost and Quality of Fuels for Electric Plants." 
Figure 17. Average Price of Natural Gas Dellvered to Consumers in the United States, 1989 - 1993

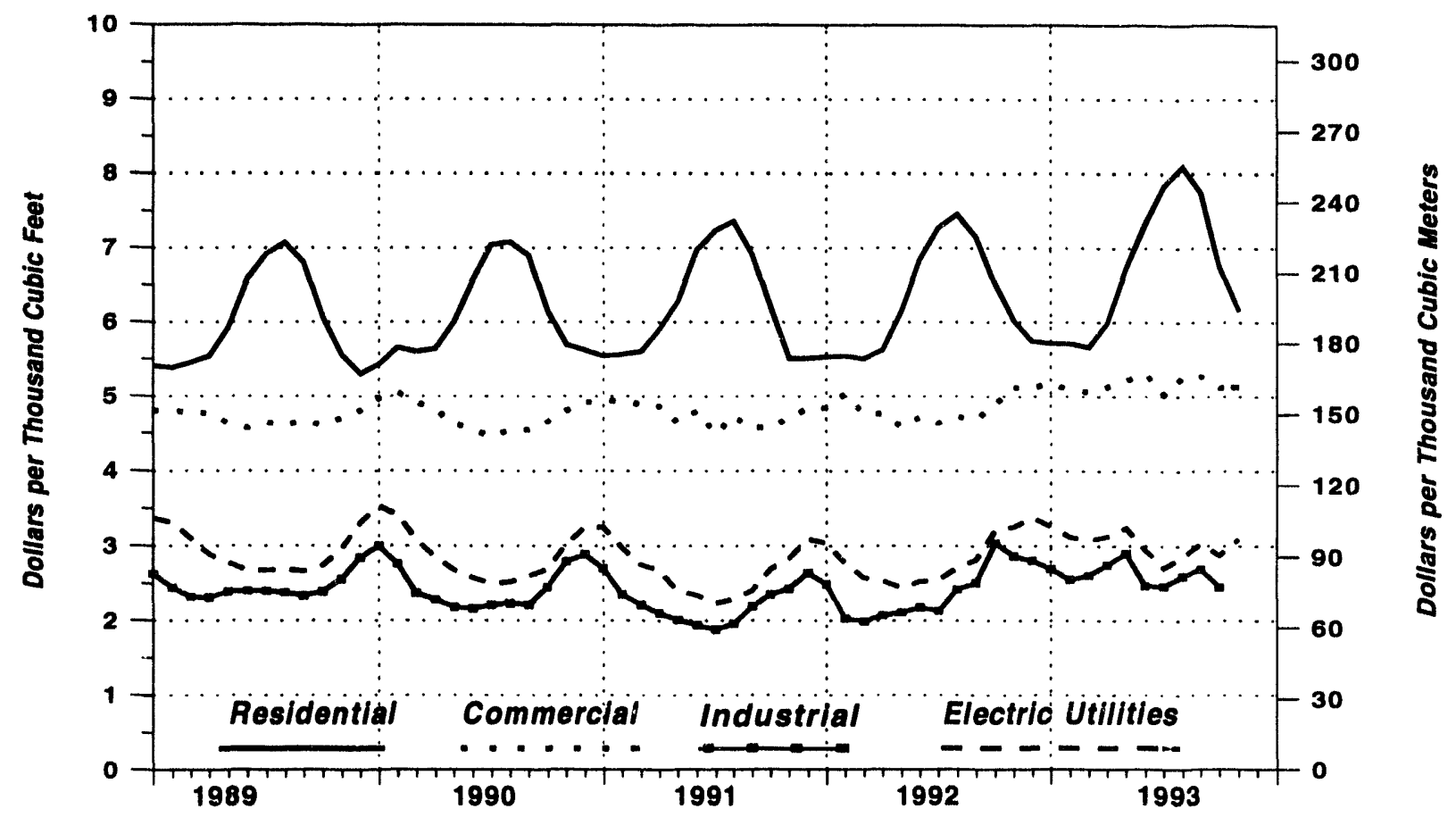

Sources: Energy Information Administration (ElA), Form EIA-857, "Monthly Report of Natural Gas Purchases and Deliveries to Consumers", Form FERC-423, "Monthly Report of Cost and Quality of Fuels for Electric Plants", Natural Gas Annual.

Figure 18. Average Price of Natural Gas in the United States, 1989 - 1993

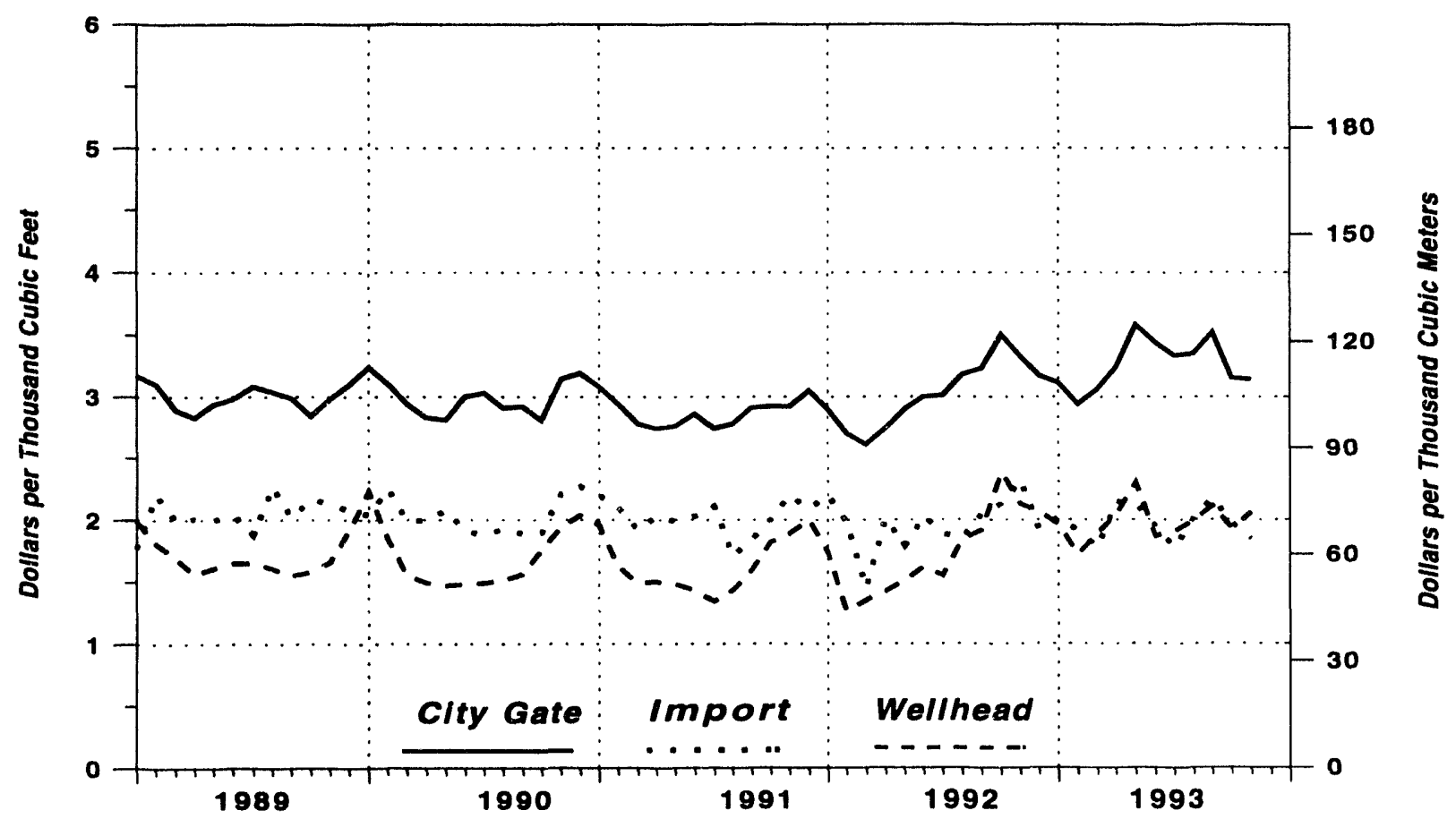

Sources: Energy Information Administration (EIA), Form EIA-857, "Monthly Report of Natural Gas Purchases and Deliveries to Consumers", Form FERC-11, "Natural Gas Pipeline Company Monthly Statement", Natural Gas Annual. 


\section{Prices}

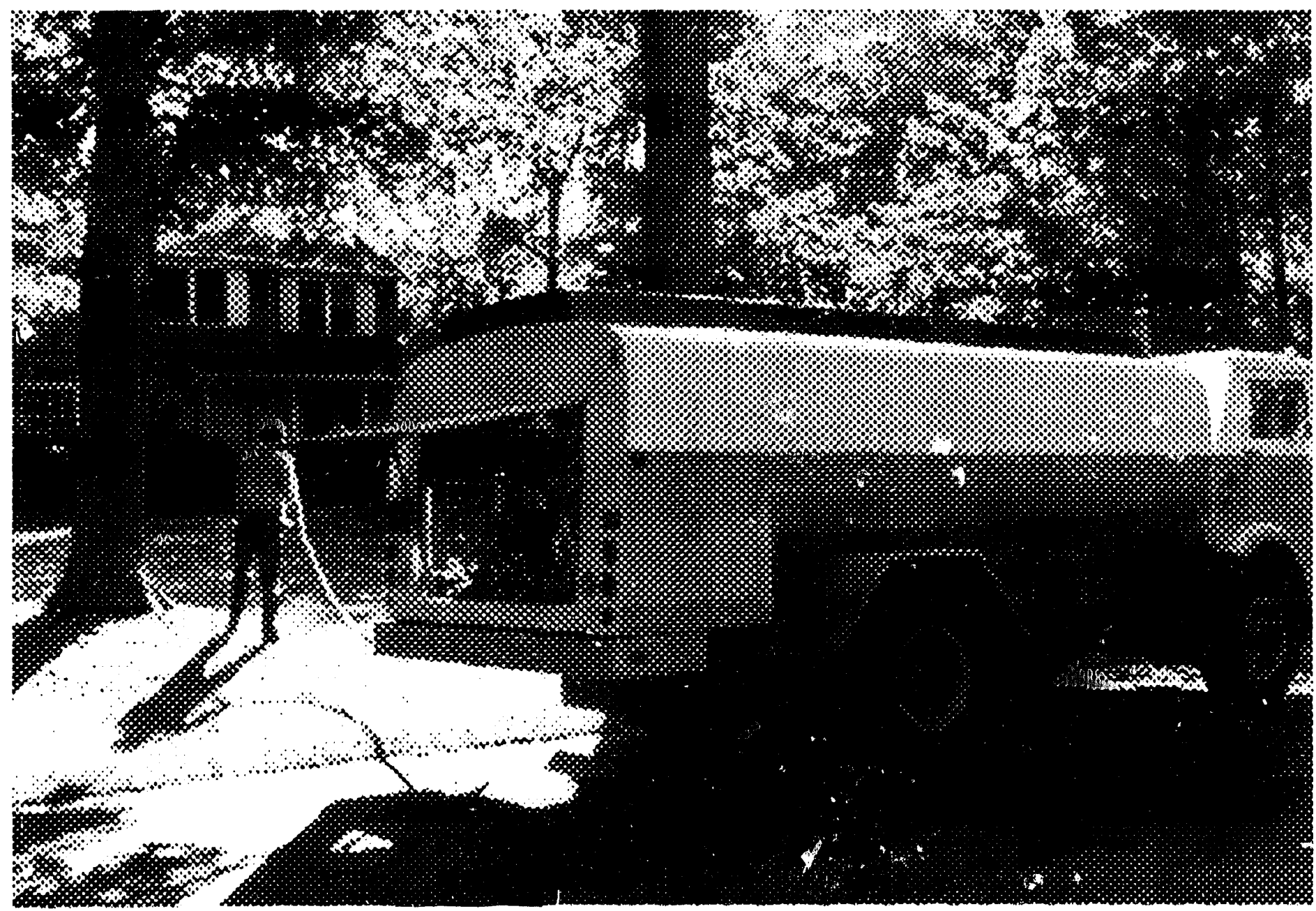

Distillate fuel oil and propane are two sources of residential heating in the United States. 
Table 7. Residentlal Heating OII Prices by Reglon and State (Cents per Gallon)

\begin{tabular}{|c|c|c|c|c|c|c|}
\hline \multirow[b]{2}{*}{ Reglon/State } & \multicolumn{6}{|c|}{ 1992/93 Heating Season } \\
\hline & October & Novomber & December & January & February & March \\
\hline বে & 972 & 983 & $97 \%$ & 075 & 976 & 98.1 \\
\hline $\begin{array}{l}\text { East Coast (PADD }) \\
\text { New England (PADD IX) } \\
\text { Central Atlantic (PADD IY) } \\
\text { Lower Atlantic (PADD IZ) }\end{array}$ & $\begin{array}{r}88.6 \\
96.3 \\
100.3 \\
93.1\end{array}$ & $\begin{array}{r}98 \% \\
96.6 \\
101.9 \\
94.6\end{array}$ & $\begin{array}{r}991 \\
95.7 \\
101.3 \\
93.8\end{array}$ & $\begin{array}{r}99.3 \\
96.3 \\
101.5 \\
93.4\end{array}$ & $\begin{array}{r}09.5 \\
96.6 \\
101.7 \\
93.3\end{array}$ & $\begin{array}{r}99.9 \\
96.8 \\
102.2 \\
93.4\end{array}$ \\
\hline Mowost (PADD II) & 89.8 & 900 & 877 & $871 \%$ & 870 & 880 \\
\hline
\end{tabular}

\begin{tabular}{|c|c|c|c|c|c|c|c|c|c|c|c|c|}
\hline \multirow[b]{2}{*}{ Reglon/State } & \multicolumn{12}{|c|}{ 1993/94 Heating Season } \\
\hline & $10 / 04$ & $10 / 18$ & $11 / 01$ & $11 / 15$ & $12 / 06$ & $12 / 20$ & $01 / 03$ & $01 / 17$ & $01 / 31$ & $02 / 07$ & $02 / 14$ & $02 / 21$ \\
\hline Averago & 08.6 & $841 \%$ & 8.8. & 847 & 038. & $026 \%$ & 821 & 847 & 966 & 98.7 & 99.5 & \\
\hline East Coest (PADDI) & $05 \%$ & 956 & 05.8 & $95 \%$ & 858 & 8\%, & 841 & 967 & 98.8 & 1010 & 1018 & \\
\hline $\begin{array}{l}\text { Now England (PADDIX) } \\
\text { Connecticut } \\
\text { Maine } \\
\text { Massachusetts } \\
\text { New Hampshire } \\
\text { Rhode Island } \\
\text { Vermont }\end{array}$ & $\begin{array}{l}91.4 \\
94.9 \\
83.2 \\
91.6 \\
86.5 \\
95.5 \\
91.3\end{array}$ & $\begin{array}{l}917 \\
95.8 \\
82.7 \\
91.6 \\
87.4 \\
95.2 \\
92.0\end{array}$ & $\begin{array}{l}91.6 \\
96.0 \\
81.0 \\
91.6 \\
88.0 \\
94.2 \\
91.9\end{array}$ & $\begin{array}{l}91.6 \\
96.0 \\
79.6 \\
92.0 \\
88.0 \\
94.2 \\
91.8\end{array}$ & $\begin{array}{l}90.5 \\
94.7 \\
78.9 \\
90.3 \\
87.4 \\
94.5 \\
92.1\end{array}$ & $\begin{array}{l}89.2 \\
94.6 \\
76.5 \\
88.7 \\
86.4 \\
92.5 \\
90.6\end{array}$ & $\begin{array}{l}891 \\
93.3 \\
79.1 \\
88.6 \\
86.2 \\
91.9 \\
90.7\end{array}$ & $\begin{array}{l}92.9 \\
96.1 \\
85.4 \\
93.5 \\
88.7 \\
93.6 \\
91.5\end{array}$ & $\begin{array}{l}95.0 \\
97.8 \\
86.5 \\
95.9 \\
92.6 \\
95.6 \\
93.0\end{array}$ & $\begin{array}{l}971 \\
99.0 \\
90.9 \\
97.9 \\
95.3 \\
97.5 \\
96.3\end{array}$ & $\begin{array}{r}98.4 \\
100.8 \\
91.8 \\
99.0 \\
96.8 \\
98.0 \\
97.2\end{array}$ & \\
\hline $\begin{array}{l}\text { Central Atjantic (PADD M) } \\
\text { Delaware } \\
\text { District of Columbia } \\
\text { Maryland } \\
\text { New Jersey } \\
\text { New York } \\
\text { Pennsylvania }\end{array}$ & $\begin{array}{r}97.4 \\
91.6 \\
105.4 \\
97.9 \\
98.1 \\
103.4 \\
86.4\end{array}$ & $\begin{array}{r}98.1 \\
92.4 \\
105.5 \\
98.1 \\
99.6 \\
103.8 \\
87.3\end{array}$ & $\begin{array}{r}98.5 \\
92.6 \\
105.5 \\
98.4 \\
99.8 \\
104.4 \\
87.6\end{array}$ & $\begin{array}{r}98.7 \\
92.6 \\
105.4 \\
98.5 \\
99.3 \\
104.7 \\
88.0\end{array}$ & $\begin{array}{r}98.5 \\
92.4 \\
105.2 \\
96.8 \\
98.6 \\
104.7 \\
87.9\end{array}$ & $\begin{array}{r}97.5 \\
92.4 \\
105.3 \\
95.4 \\
97.8 \\
103.9 \\
86.7\end{array}$ & $\begin{array}{r}974 \\
92.4 \\
104.3 \\
95.3 \\
97.6 \\
103.8 \\
86.5\end{array}$ & $\begin{array}{r}99.3 \\
95.6 \\
105.3 \\
98.9 \\
100.1 \\
105.3 \\
88.4\end{array}$ & $\begin{array}{r}101.4 \\
97.5 \\
107.1 \\
101.4 \\
102.1 \\
106.5 \\
92.2\end{array}$ & $\begin{array}{r}103.8 \\
98.4 \\
108.4 \\
103.6 \\
104.6 \\
108.8 \\
94.7\end{array}$ & $\begin{array}{r}104.5 \\
97.7 \\
109.1 \\
103.9 \\
105.5 \\
109.3 \\
95.9\end{array}$ & \\
\hline $\begin{array}{l}\text { Lower Atlantlo (PADD IZ) } \\
\text { North Carolina } \\
\text { Virginia }\end{array}$ & $\begin{array}{l}89.0 \\
89.5 \\
88.4\end{array}$ & $\begin{array}{l}89.6 \\
89.8 \\
89.4\end{array}$ & $\begin{array}{l}89.3 \\
89.1 \\
89.5\end{array}$ & $\begin{array}{l}89.6 \\
89.5 \\
89.7\end{array}$ & $\begin{array}{l}89.0 \\
88.9 \\
89.1\end{array}$ & $\begin{array}{l}87.5 \\
87.0 \\
88.0\end{array}$ & $\begin{array}{l}875 \\
87.1 \\
88.0\end{array}$ & $\begin{array}{l}89.8 \\
89.4 \\
90.3\end{array}$ & $\begin{array}{l}92.7 \\
91.8 \\
93.9\end{array}$ & $\begin{array}{l}937 \\
93.2 \\
94.2\end{array}$ & $\begin{array}{l}93.6 \\
93.0 \\
94.2\end{array}$ & \\
\hline $\begin{array}{l}\text { Mlowast (PADDII) } \\
\text { Indiana } \\
\text { lowa } \\
\text { Michigan } \\
\text { Minriasota } \\
\text { Ohio } \\
\text { Wisconsin }\end{array}$ & $\begin{array}{l}85.8 \\
85.4 \\
79.1 \\
86.6 \\
88.9 \\
84.2 \\
85.4\end{array}$ & $\begin{array}{c}89.3 \\
88.3 \\
\text { NA } \\
89.6 \\
92.5 \\
86.5 \\
88.8\end{array}$ & $\begin{array}{l}88.2 \\
87.0 \\
82.7 \\
90.0 \\
91.9 \\
86.2 \\
87.1\end{array}$ & $\begin{array}{c}87.5 \\
86.0 \\
N A \\
89.3 \\
90.5 \\
85.3 \\
85.9\end{array}$ & $\begin{array}{l}85.0 \\
82.8 \\
76.1 \\
87.2 \\
88.9 \\
82.4 \\
85.2\end{array}$ & $\begin{array}{c}83.0 \\
B 1.1 \\
\text { NA } \\
84.6 \\
85.6 \\
79.5 \\
82.7\end{array}$ & $\begin{array}{l}80.9 \\
79.8 \\
66.7 \\
83.6 \\
84.3 \\
78.5 \\
81.8\end{array}$ & $\begin{array}{c}83.5 \\
82.1 \\
\text { NA } \\
84.5 \\
85.2 \\
82.2 \\
82.8\end{array}$ & $\begin{array}{l}84.0 \\
84.0 \\
69.9 \\
87.5 \\
85.9 \\
83.9 \\
83.6\end{array}$ & $\begin{array}{c}85.6 \\
86.4 \\
70.9 \\
89.5 \\
86.3 \\
86.0 \\
85.2\end{array}$ & $\begin{array}{l}85.9 \\
85.5 \\
71.3 \\
89.4 \\
87.0 \\
86.3 \\
85.9\end{array}$ & \\
\hline
\end{tabular}

NA=Not available.

$P=$ Preliminary data.

$R=$ Revised data.

Note: Due to severe weather conditions and stock drawdowns, EIA initlated weekly collection of State heating oil prices to monitor prices at the retail level.

Source: Based on data collected by State Energy Offices. 
Figure 19. Residential Heating Oil Prices, New England

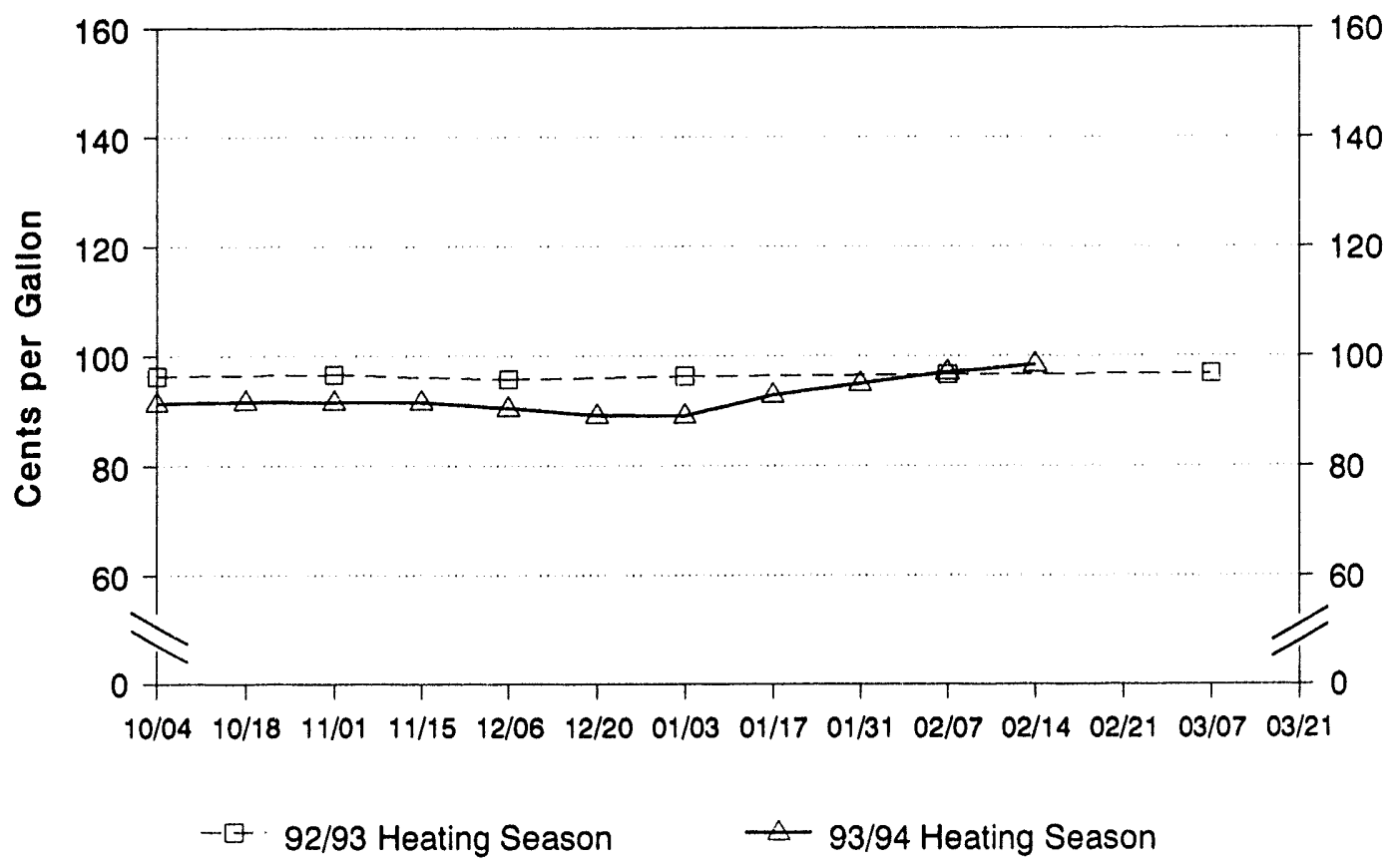

Source: Based on data collected by State Energy Offices.

Figure 20. Residential Heating Oil Prices, Central Atlantic

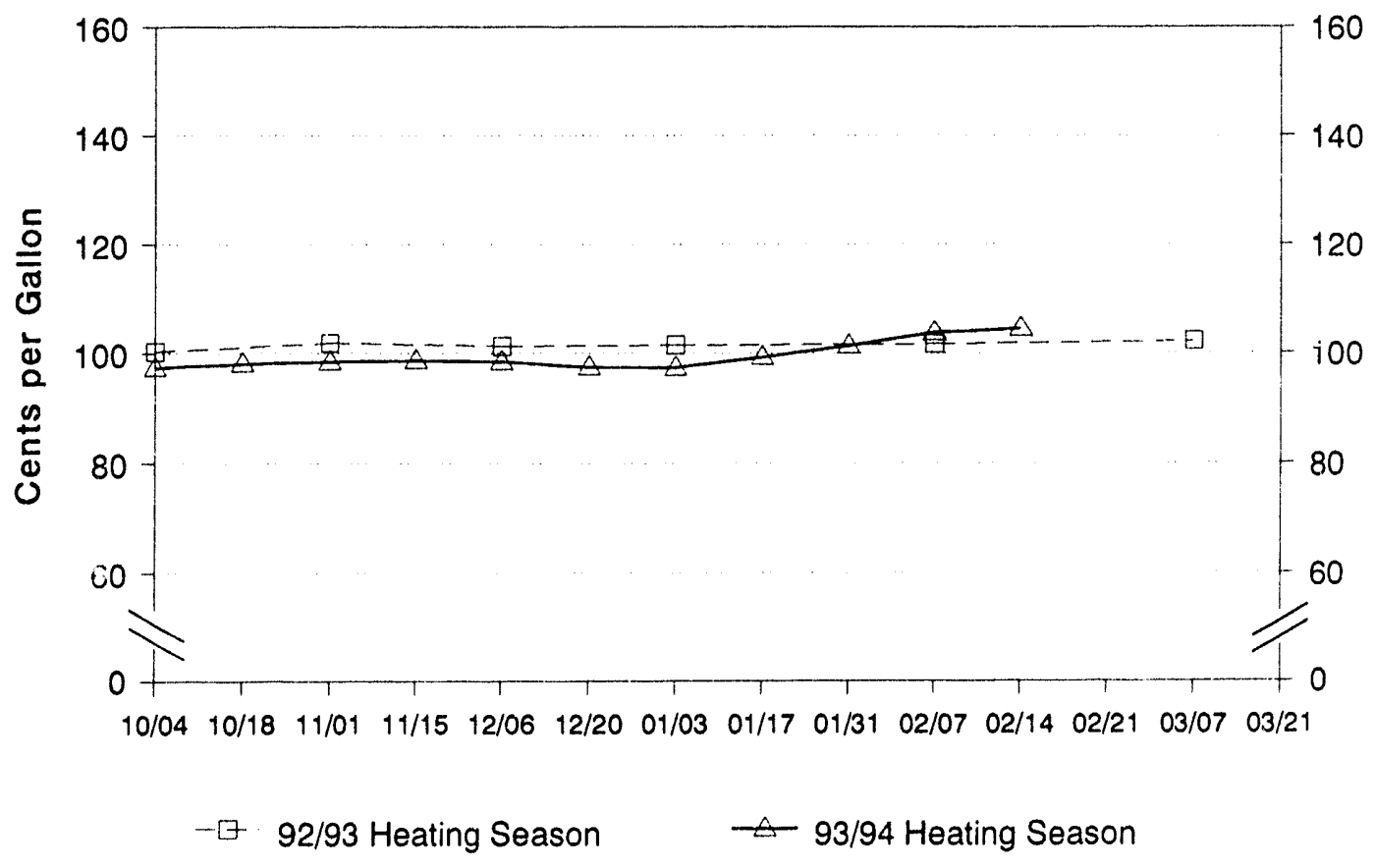

Source: Based on data collected by State Energy Offices. 
Figure 21. Residential Heating Oil Prices, Lower Atlantic

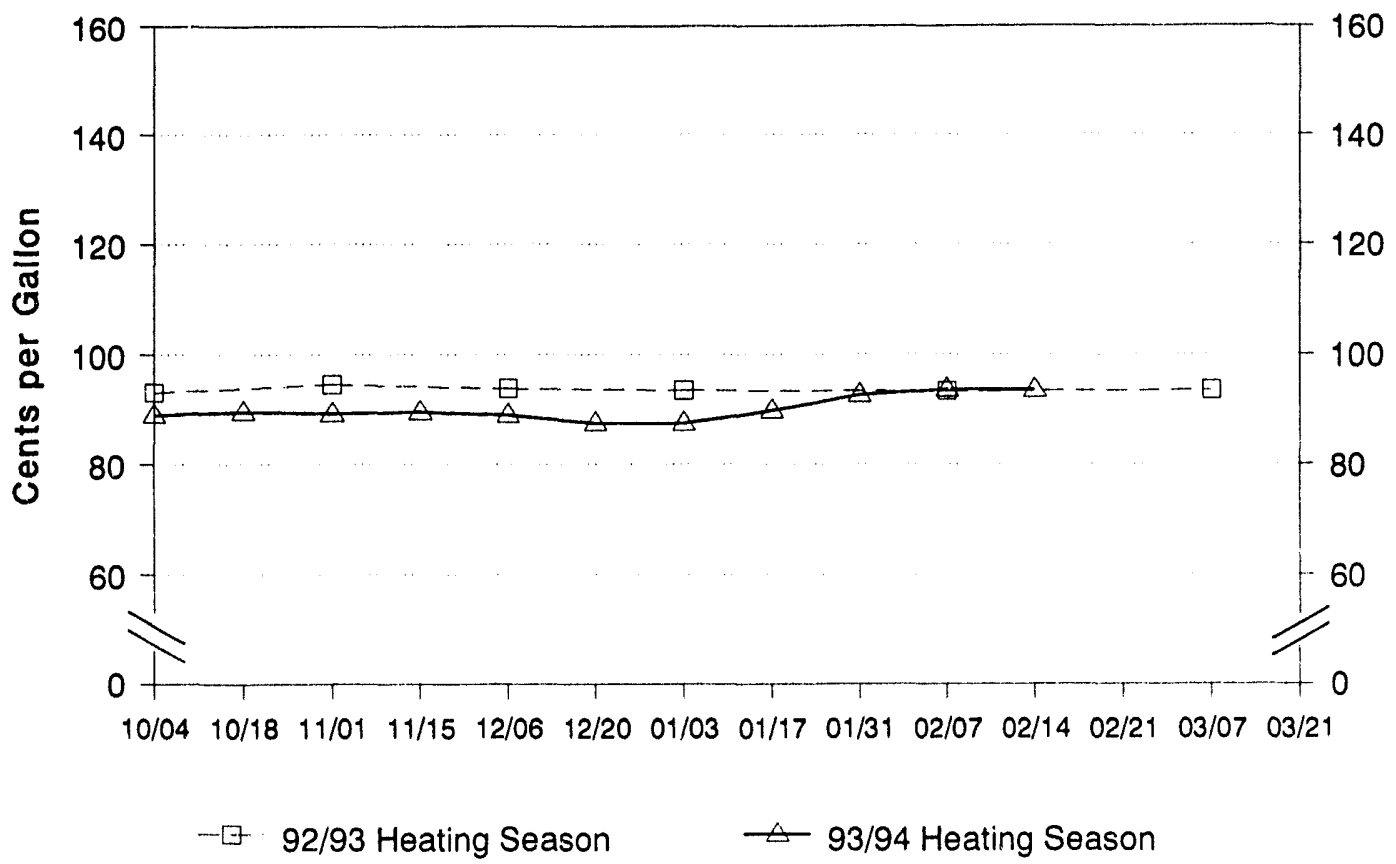

Source: Based on data collected by State Energy Offices.

Figure 22. Residential Heating Oil Prices, Midwest

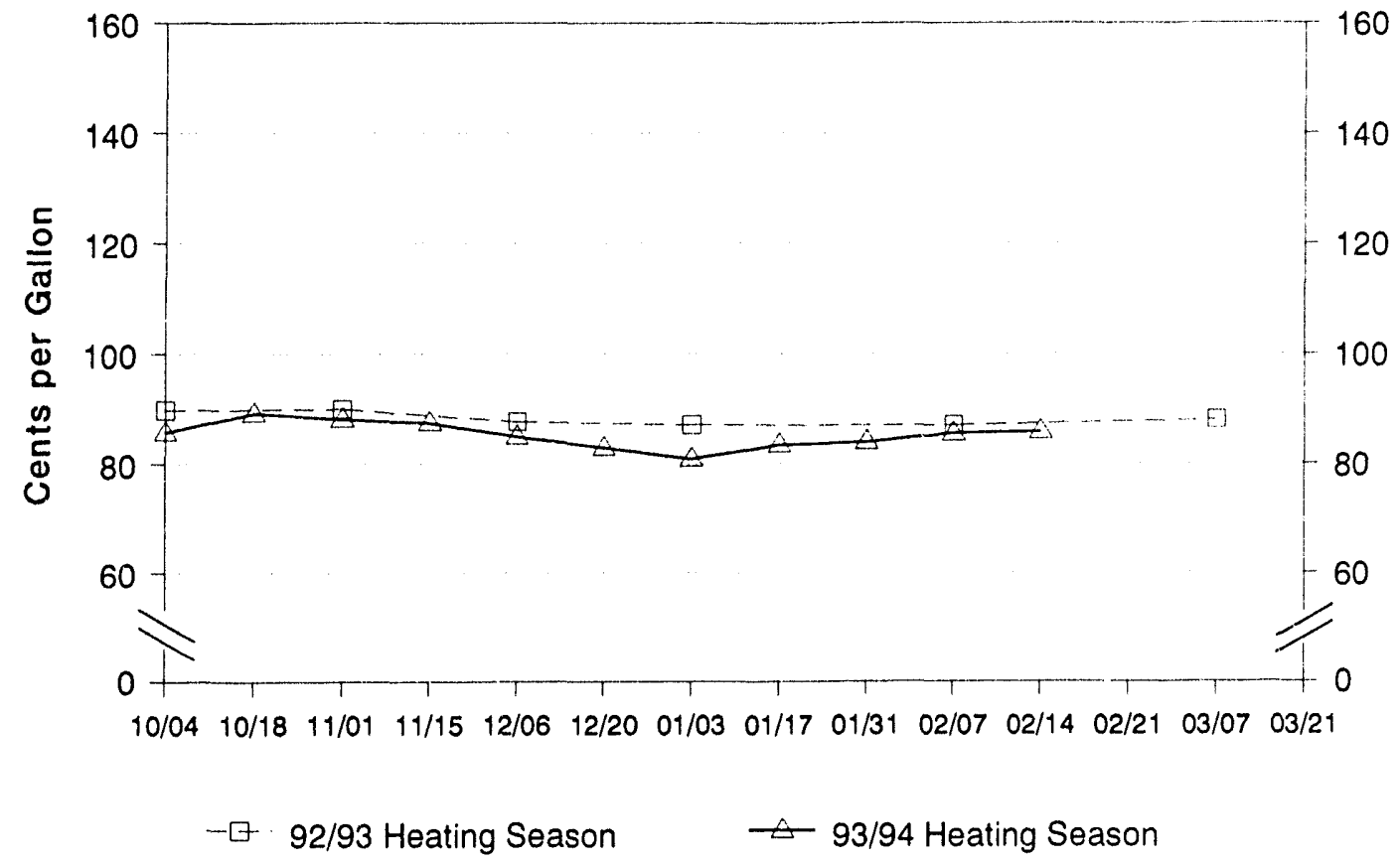

Source: Based on data collected by State Energy Offices. 
Table 8. Residential Propane Prices by Region and State (Cents per Gallon)

\begin{tabular}{|c|c|c|c|c|c|c|}
\hline \multirow[b]{2}{*}{ Reglon/State } & \multicolumn{6}{|c|}{ 1992/93 Heating Season } \\
\hline & October & November & December & January & February & March \\
\hline Average & 858 & 672 & 89.5 & 979 & 94.6 & 95.6 \\
\hline East Coast (PADD) & $115 \%$ & 1154 & 115.7 & 116.7 & 116.9 & 118.1 \\
\hline New England (PADD IX) & 116.9 & 116.6 & 116.4 & 117.4 & 118.3 & 119.3 \\
\hline Central Atlantic (PADD IY) & 125.2 & 125.6 & 126.4 & 127.3 & 127.0 & 129.8 \\
\hline Lower Atlantic (PADD IZ) & 100.2 & 100.5 & 100.8 & 102.2 & 102.0 & 101.3 \\
\hline MIdWOst (PADD II) & 1022 & 721 & 15.3 & 87.7 & 82.3 & 83.3 \\
\hline
\end{tabular}

\begin{tabular}{|c|c|c|c|c|c|c|c|c|c|c|c|c|}
\hline \multirow[b]{2}{*}{ Reglon/State } & \multicolumn{12}{|c|}{ 1993/94 Heating Season } \\
\hline & $10 / 04$ & $10 / 18$ & $11 / 01$ & $11 / 15$ & $12 / 06$ & $12 / 20$ & $01 / 03$ & $01 / 17$ & $01 / 31$ & $02 / 07$ & $02 / 14$ & $02 / 21$ \\
\hline Averago & 870 & 872 & 876 & 88.0 & 88,2 & 88.0 . & 88.0 & 88.4 & 89.6 & 90.5 & 90.9 & \\
\hline East Coast (PADO) & 1101 & 1102 & 1103 & 1107 & 1109 & 1110 & 1111 & 1118 & 113.5 & 114.6 & 115.0 & \\
\hline Now england (PADO IX) & 115.5 & 1156 & 115.6 & 115.6 & 1150 & $1161 \%$ & 116.1 & 116. & 116.8 & 1177 & 117.8 & \\
\hline Connecticut & 112.2 & 112.9 & 111.9 & 112.7 & 112.5 & 113.8 & 114.4 & 114.3 & 115.5 & 115.3 & 115.9 & \\
\hline Maine & 125.8 & 125.5 & 125.6 & 125.4 & 125.4 & 125.3 & 125.3 & 125.3 & 125.4 & 126.0 & 123.6 & \\
\hline Massachusetts & 113.8 & 114.4 & 114.8 & 115.2 & 115.5 & 115.4 & 115.4 & 115.1 & 115.5 & 115.8 & 117.3 & \\
\hline New Hampshire & 110.2 & 108.9 & 109.0 & 109.0 & 109.8 & 109.8 & 109.8 & 109.9 & 110.6 & 113.3 & 113.5 & \\
\hline Rhode Island & 129.3 & 130.8 & 130.8 & 130.5 & 130.4 & 130.3 & 129.5 & 129.5 & 129.6 & 130.0 & 130.6 & \\
\hline Vermont & 114.7 & 114.5 & 114.6 & 114.7 & 114.5 & 114.4 & 114.1 & 114.4 & 115.6 & 117.1 & 117.4 & \\
\hline Central Atlantlc (PADD M & 118.1 & 118.3 & 1182 & 118.4 & 118.7 & 118.7 & 1187 & 119.9 & 121.0 & 122.7 & 123.4 & \\
\hline Delaware & 110.3 & 111.3 & 111.3 & 111.2 & 111.2 & 111.3 & 111.4 & 113.8 & 118.1 & 120.0 & 122.8 & \\
\hline Maryland & 118.7 & 119.1 & 119.1 & 119.2 & 119.9 & 119.9 & 119.6 & 120.3 & 125.6 & 127.9 & 128.0 & \\
\hline New Jersey & 118.6 & 118.6 & 119.1 & 119.3 & 119.3 & 119.3 & 119.6 & 119.8 & 120.0 & 122.6 & 124.7 & \\
\hline New York & 123.3 & 123.4 & 122.5 & 123.1 & 123.2 & 123.5 & 123.7 & 124.4 & 125.1 & 126.4 & 126.9 & \\
\hline Pennsylvania & 113.8 & 113.8 & 113.8 & 114.0 & 114.3 & 114.2 & 114.2 & 116.1 & 115.5 & 116.9 & 116.8 & \\
\hline Lower Atlantic (PADD IZ) & 95.2 & 95.3 & 95.6 & 96.3 & 96,5 & 96,8 & 96.8 & 97.9 & 100.8 & 101.5 & 101.7 & \\
\hline North Carolina & 92.7 & 92.7 & 93.1 & 94.0 & 94.3 & 94.7 & 94.7 & 95.9 & 99.2 & 99.9 & 100.0 & \\
\hline Virginia & 104.8 & 104.9 & 104.9 & 104.8 & 104.8 & 104.7 & 104.8 & 105.4 & 107.3 & 107.4 & 107.9 & \\
\hline Midwest (PADD II) & 74.0 & 74.3 & 74.7 & 74.9 & 75.2 & 74.8 & 74.7 & 75.1 & 75.9 & 76.7 & 76.8 & \\
\hline Indiana & 79.8 & 81.5 & 82.5 & 83.3 & 83.5 & 84.2 & 83.8 & 84.7 & 87.3 & 87.6 & 87.2 & \\
\hline lowa & 60.5 & 60.0 & 60.4 & 61.1 & 61.3 & 59.9 & 59.3 & 59.3 & 59.1 & 59.8 & 60.3 & \\
\hline Kansas & 62.0 & 62.1 & 62.2 & 62.8 & 62.8 & 61.3 & 61.2 & 61.2 & 61.2 & 61.6 & 61.9 & \\
\hline Michigan & 84.0 & 84.4 & 84.5 & 84.9 & 85.0 & 85.0 & 85.0 & 85.4 & 87.0 & 87.8 & 88.3 & \\
\hline Minnesota & 75.8 & 77.0 & 77.1 & 77.2 & 77.6 & 76.6 & 77.3 & 77.9 & 78.9 & 79.3 & 79.2 & \\
\hline Missouri & 70.7 & 70.6 & 72.5 & 72.8 & 73.6 & 73.5 & 73.4 & 73.8 & 73.9 & 74.5 & 74.3 & \\
\hline North Dakota & 61.4 & 62.2 & 62.4 & 62.5 & 63.0 & 62.7 & 62.4 & 62.8 & 63.4 & 64.1 & 64.2 & \\
\hline Ohio & 87.4 & 87.7 & 87.8 & 87.3 & 87.0 & 87.2 & 87.6 & 88.2 & 89.3 & 89.9 & 90.0 & \\
\hline South Dakota & 62.1 & 63.9 & 63.9 & 64.4 & 64.4 & 63.7 & 63.1 & 63.8 & 63.4 & 64.0 & 64.0 & \\
\hline Wisconsin & 77.3 & 76.8 & 76.5 & 76.3 & 76.8 & 76.7 & 76.2 & 76.7 & 77.9 & 79.5 & 79.8 & \\
\hline
\end{tabular}

$P=$ Preliminary data.

R=Revised data.

Note: Due to severe weather conditions and stock drawdowns, EIA Initlated weekly collection of State propane prices to monitor prices at the retail level.

Source: Based on data collected by State Energy Offices. 
Figure 23. Residential Propane Prices, New England

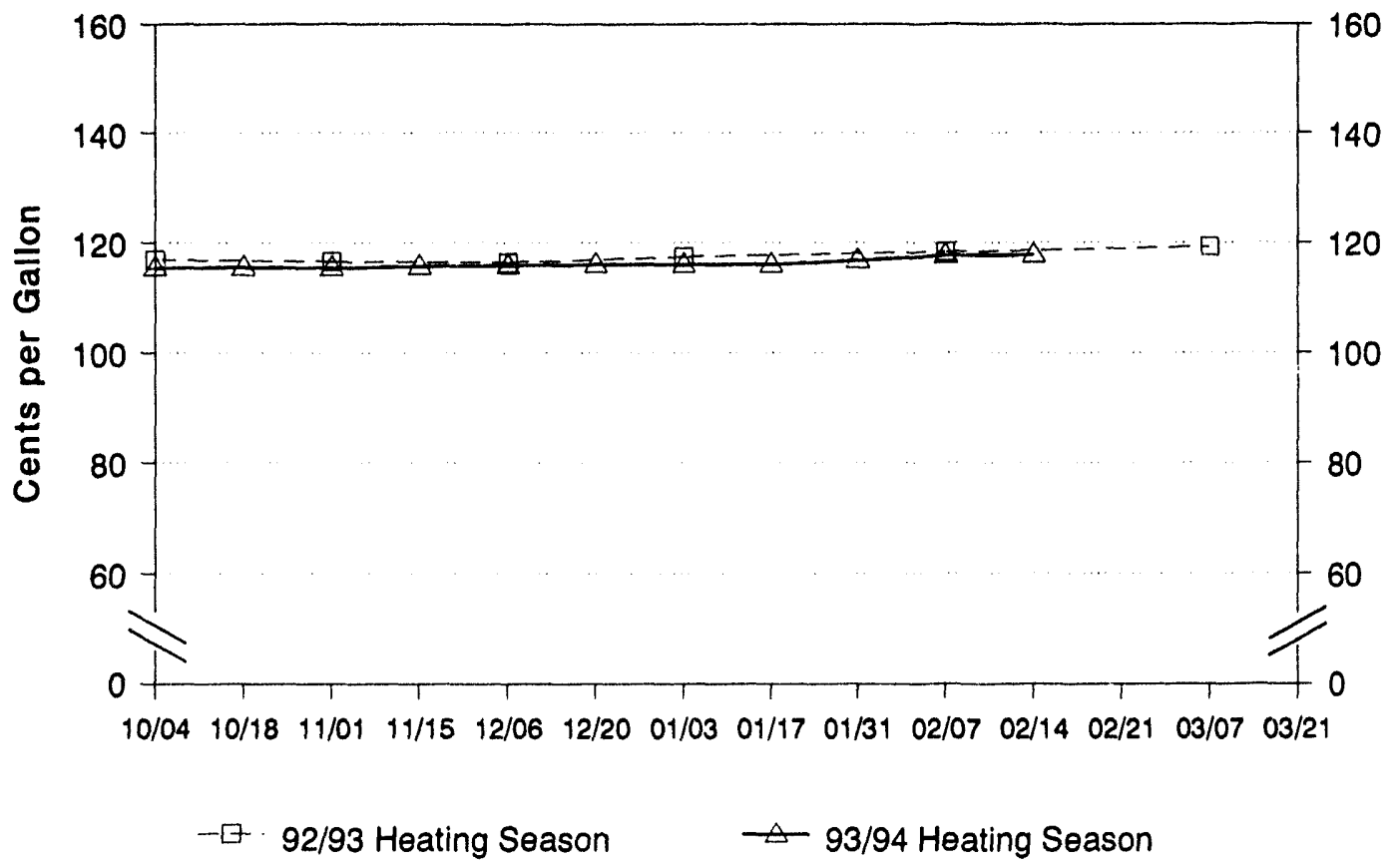

Source: Based on data collected by State Energy Offices.

Figure 24. Residential Propane Prices, Central Atlantic

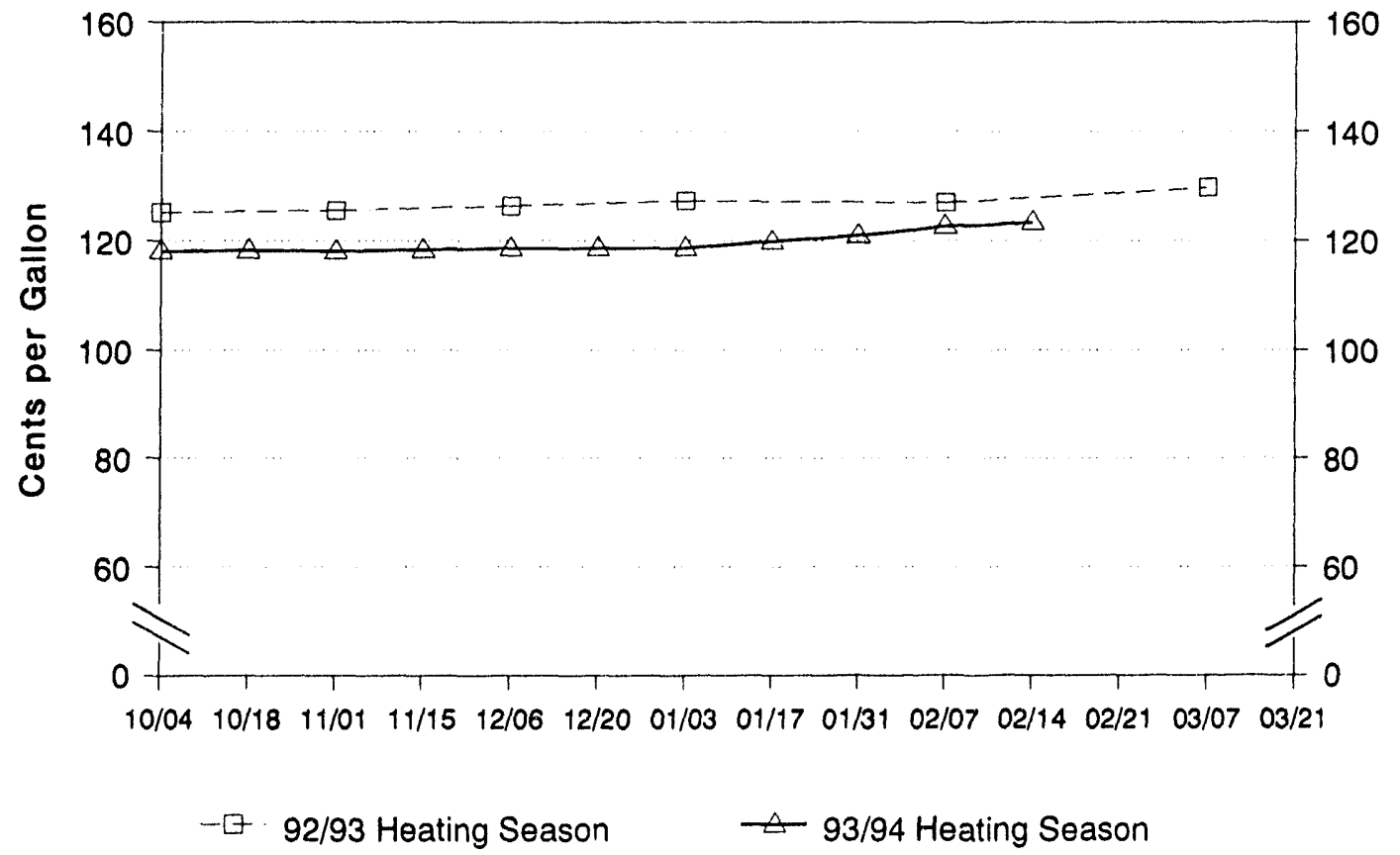

Source: Based on data collected by State Energy Offices. 
Figure 25. Residential Propane Prices, Lower Atlantic

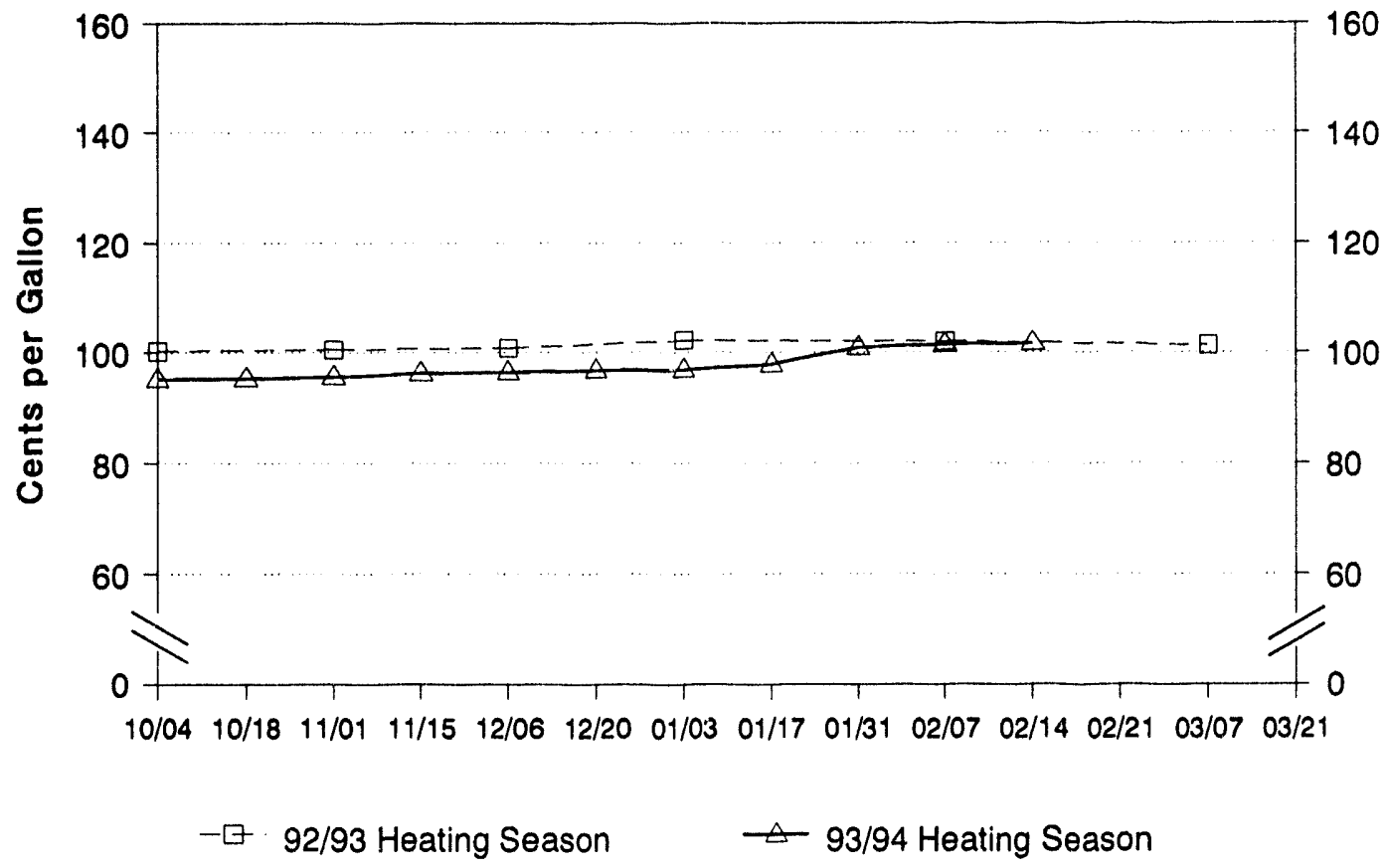

Source: Based on data collected by State Energy Offices.

Figure 26. Residential Propane Prices, Midwest

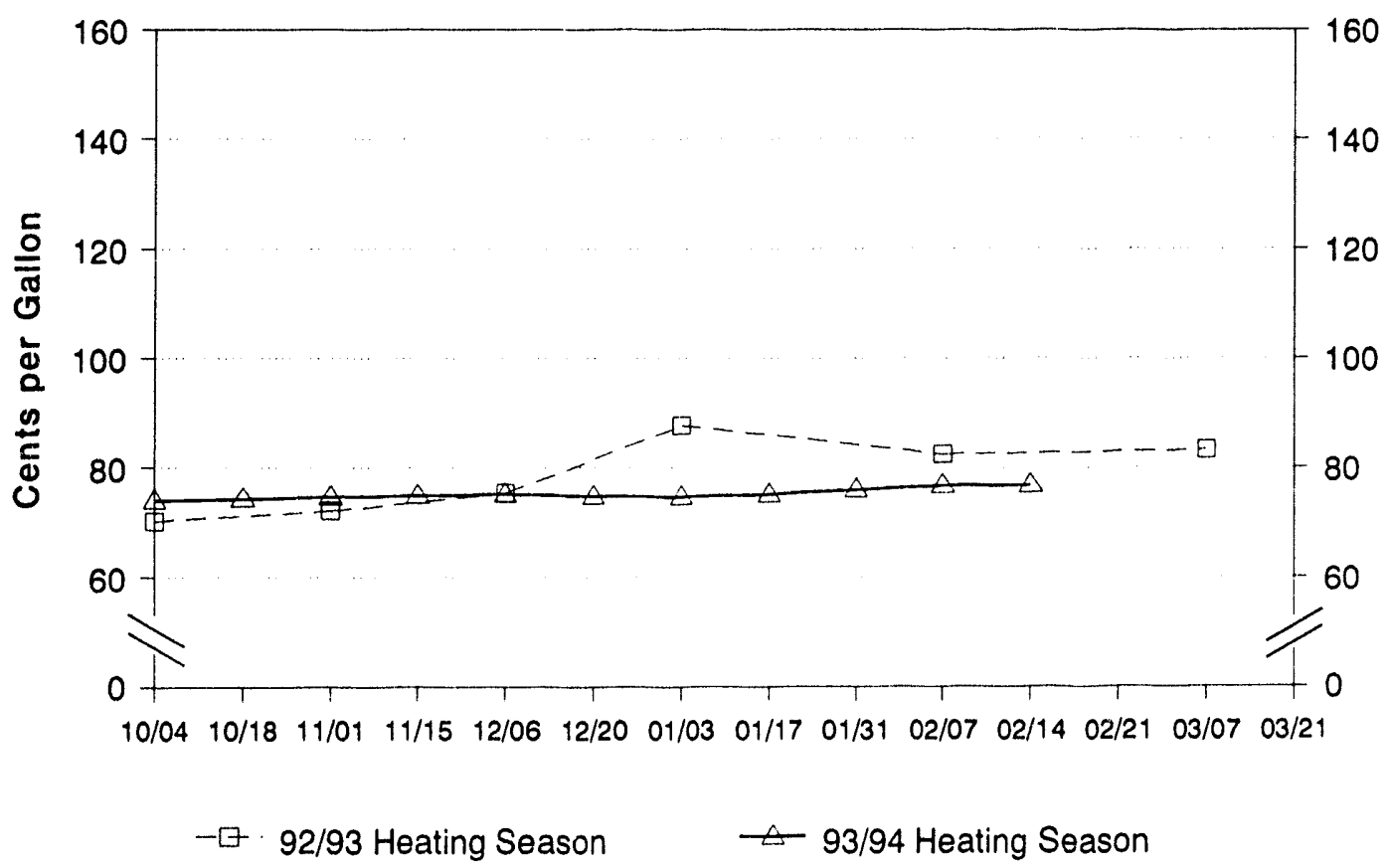

Source: Based on data collected by State Energy Offices. 
Table 9. Wholesale Heating Oil Prices by Reglon and State (Cents per Gallon)

\begin{tabular}{|c|c|c|c|c|c|c|}
\hline \multirow[b]{2}{*}{ Reglon/State } & \multicolumn{6}{|c|}{ 1992/93 Heating Season } \\
\hline & October & November & December & January & February & March \\
\hline Avarage $/$,, প. & $867 \%$ & 61.6 & 573 & 56.3 & 58,3 & 610 \\
\hline $\begin{array}{l}\text { East Coast (PADD ) } \\
\text { New England (PADD IX) } \\
\text { Central Atlantic (PADD IY) } \\
\text { Lower Atlantic (PADD IZ) }\end{array}$ & $\begin{array}{l}66.8 \\
68.2 \\
66.2 \\
65.9\end{array}$ & $\begin{array}{l}61.9 \\
64.2 \\
60.9 \\
60.0\end{array}$ & $\begin{array}{l}581 \\
60.4 \\
57.1 \\
55.8\end{array}$ & $\begin{array}{l}56.9 \\
59.4 \\
55.8 \\
54.8\end{array}$ & $\begin{array}{l}58.7 \\
60.0 \\
58.3 \\
57.1\end{array}$ & $\begin{array}{l}61,1 \\
62.3 \\
60.7 \\
59.4\end{array}$ \\
\hline MIdwest (PADDII) $/$, & 66,6 & 607 & 54,8 & 54.4 & 57.3 & 60.4 \\
\hline
\end{tabular}

\begin{tabular}{|c|c|c|c|c|c|c|c|c|c|c|c|c|}
\hline \multirow[b]{2}{*}{ Region/State } & \multicolumn{12}{|c|}{ 1993/94 Heating Season } \\
\hline & $10 / 04$ & $10 / 18$ & $11 / 01$ & $11 / 15$ & $12 / 06$ & $12 / 20$ & $01 / 03$ & $01 / 17$ & $01 / 31$ & $02 / 07$ & $02 / 14$ & $02 / 21$ \\
\hline Averago & 588 & 58.9 & 54.7 & 53.8 & 48.1 & 45.2 & 50.6 & 56.3 & 57,3 & 61.6 & 61.2 & \\
\hline East Coast (PADD ) & 680 & 575 & 54.3 & 63.7 & 48.1 & 45.9 & 51.7 & 58.0 & 58.9 & 63.6 & 63.7 & \\
\hline $\begin{array}{l}\text { New England (PADOIX) } \\
\text { Connecticut } \\
\text { Maine } \\
\text { Massachusetts } \\
\text { New Hampshire } \\
\text { Rhode Island } \\
\text { Vermont }\end{array}$ & $\begin{array}{l}58.5 \\
58.9 \\
59.3 \\
58.4 \\
57.8 \\
58.3 \\
\text { NA }\end{array}$ & $\begin{array}{l}58.2 \\
57.8 \\
59.6 \\
58.3 \\
57.0 \\
57.9 \\
61.1\end{array}$ & $\begin{array}{l}55.1 \\
54.4 \\
56.5 \\
54.9 \\
55.5 \\
54.8 \\
57.6\end{array}$ & $\begin{array}{l}54.8 \\
54.3 \\
56.2 \\
54.6 \\
55.3 \\
54.3 \\
57.4\end{array}$ & $\begin{array}{l}49.2 \\
48.9 \\
50.4 \\
48.7 \\
51.0 \\
48.7 \\
51.1\end{array}$ & $\begin{array}{l}47.1 \\
46.9 \\
48.2 \\
46.8 \\
47.6 \\
46.4 \\
49.6\end{array}$ & $\begin{array}{l}53.0 \\
52.3 \\
54.1 \\
53.2 \\
53.6 \\
51.7 \\
56.3\end{array}$ & $\begin{array}{l}59.7 \\
57.9 \\
61.5 \\
60.0 \\
61.2 \\
58.8 \\
62.1\end{array}$ & $\begin{array}{l}60.8 \\
59.5 \\
60.7 \\
61.6 \\
61.0 \\
60.3 \\
\text { NA }\end{array}$ & $\begin{array}{l}65.7 \\
64.4 \\
66.2 \\
66.1 \\
66.5 \\
65.3 \\
\text { NA }\end{array}$ & $\begin{array}{l}65.5 \\
64.4 \\
67.3 \\
65.6 \\
66.3 \\
64.9 \\
\text { NA }\end{array}$ & \\
\hline $\begin{array}{l}\text { Central Atlantlo }(\mathrm{PADO} \mathrm{M}) \\
\text { Delaware } \\
\text { District of Columbia } \\
\text { Maryland } \\
\text { New Jersey } \\
\text { New York } \\
\text { Pennsylvania }\end{array}$ & $\begin{array}{l}581 \\
57.0 \\
56.9 \\
56.9 \\
57.6 \\
58.7 \\
58.8\end{array}$ & $\begin{array}{l}57 . \\
56.2 \\
56.8 \\
56.3 \\
55.9 \\
58.6 \\
58.5\end{array}$ & $\begin{array}{l}541 \\
53.5 \\
53.1 \\
52.9 \\
52.7 \\
55.9 \\
54.7\end{array}$ & $\begin{array}{c}53.3 \\
52.7 \\
52.5 \\
52.1 \\
52.4 \\
54.8 \\
53.5\end{array}$ & $\begin{array}{l}47.7 \\
46.4 \\
47.2 \\
46.3 \\
46.8 \\
49.5 \\
47.7\end{array}$ & $\begin{array}{l}45.4 \\
44.3 \\
45.5 \\
45.1 \\
44.4 \\
46.9 \\
45.5\end{array}$ & $\begin{array}{l}51.3 \\
50.8 \\
50.6 \\
50.3 \\
50.6 \\
52.8 \\
50.8\end{array}$ & $\begin{array}{l}57.6 \\
57.3 \\
58.0 \\
62.8 \\
56.6 \\
58.1 \\
57.2\end{array}$ & $\begin{array}{l}58.7 \\
56.8 \\
57.5 \\
63.7 \\
56.9 \\
60.5 \\
58.4\end{array}$ & $\begin{array}{l}63.6 \\
62.8 \\
62.6 \\
63.7 \\
62.8 \\
65.5 \\
62.4\end{array}$ & $\begin{array}{l}64.0 \\
63.5 \\
61.6 \\
62.0 \\
64.1 \\
66.1 \\
61.7\end{array}$ & \\
\hline $\begin{array}{l}\text { Lower Atlantlc (PADD I Z) } \\
\text { North Carolina } \\
\text { Virginia }\end{array}$ & $\begin{array}{l}571 \\
57.6 \\
56.7\end{array}$ & $\begin{array}{l}56.5 \\
57.1 \\
56.0\end{array}$ & $\begin{array}{l}53.2 \\
53.6 \\
52.8\end{array}$ & $\begin{array}{l}52.4 \\
52.7 \\
52.2\end{array}$ & $\begin{array}{l}46.4 \\
46.6 \\
46.2\end{array}$ & $\begin{array}{l}45.1 \\
45.4 \\
44.8\end{array}$ & $\begin{array}{l}49.6 \\
49.8 \\
49.4\end{array}$ & $\begin{array}{l}54.6 \\
54.7 \\
54.5\end{array}$ & $\begin{array}{l}54.2 \\
54.0 \\
54.3\end{array}$ & $\begin{array}{l}57.7 \\
57.8 \\
57.6\end{array}$ & $\begin{array}{l}56.5 \\
56.6 \\
56.4\end{array}$ & \\
\hline $\begin{array}{l}\text { Midwest (PADD II) } \\
\text { Illinois } \\
\text { Indiana } \\
\text { lowa } \\
\text { Kansas } \\
\text { Michigan } \\
\text { Minnesota } \\
\text { Missouri } \\
\text { North Dakota } \\
\text { Ohio } \\
\text { South Dakota } \\
\text { Wisconsin }\end{array}$ & $\begin{array}{l}611 \\
61.1 \\
58.9 \\
64.1 \\
64.7 \\
57.2 \\
63.4 \\
61.9 \\
65.8 \\
60.9 \\
67.7 \\
61.5\end{array}$ & $\begin{array}{l}63.6 \\
62.1 \\
59.3 \\
67.1 \\
68.9 \\
58.9 \\
68.5 \\
66.3 \\
73.8 \\
61.9 \\
76.2 \\
64.8\end{array}$ & $\begin{array}{l}56.0 \\
54.8 \\
53.0 \\
57.6 \\
57.4 \\
52.2 \\
60.1 \\
56.6 \\
68.6 \\
55.8 \\
69.7 \\
56.3\end{array}$ & $\begin{array}{l}54.1 \\
52.8 \\
51.4 \\
56.1 \\
56.3 \\
50.4 \\
58.5 \\
53.5 \\
61.6 \\
54.0 \\
64.2 \\
55.3\end{array}$ & $\begin{array}{l}48.2 \\
47.1 \\
45.4 \\
50.4 \\
50.5 \\
45.8 \\
52.0 \\
47.2 \\
54.8 \\
48.2 \\
53.4 \\
49.2\end{array}$ & $\begin{array}{l}42.8 \\
41.4 \\
40.7 \\
45.4 \\
44.5 \\
40.3 \\
45.9 \\
42.9 \\
48.6 \\
42.9 \\
46.8 \\
43.6\end{array}$ & $\begin{array}{l}47.0 \\
45.7 \\
45.6 \\
49.5 \\
48.8 \\
44.3 \\
50.2 \\
47.3 \\
51.5 \\
46.9 \\
49.2 \\
47.6\end{array}$ & $\begin{array}{l}50.9 \\
50.4 \\
48.8 \\
51.9 \\
51.6 \\
48.8 \\
52.8 \\
51.0 \\
54.5 \\
52.2 \\
51.2 \\
51.2\end{array}$ & $\begin{array}{l}51.7 \\
51.4 \\
50.2 \\
51.7 \\
51.2 \\
50.3 \\
53.3 \\
51.6 \\
55.4 \\
53.5 \\
49.2 \\
51.9\end{array}$ & $\begin{array}{l}54.8 \\
54.0 \\
54.2 \\
53.5 \\
52.6 \\
53.8 \\
55.5 \\
54.3 \\
56.8 \\
57.0 \\
54.0 \\
55.3\end{array}$ & $\begin{array}{l}52.7 \\
51.8 \\
51.3 \\
52.1 \\
51.2 \\
51.7 \\
53.4 \\
52.6 \\
54.9 \\
55.7 \\
52.2 \\
52.5\end{array}$ & \\
\hline
\end{tabular}

NA $=$ Not available.

$P=$ Preliminary data.

Source: Based on terminal quotes collected by the Computer Petroleum Corporation, Inc. 
Figure 27. Wholesale Heating Oil Prices, New England

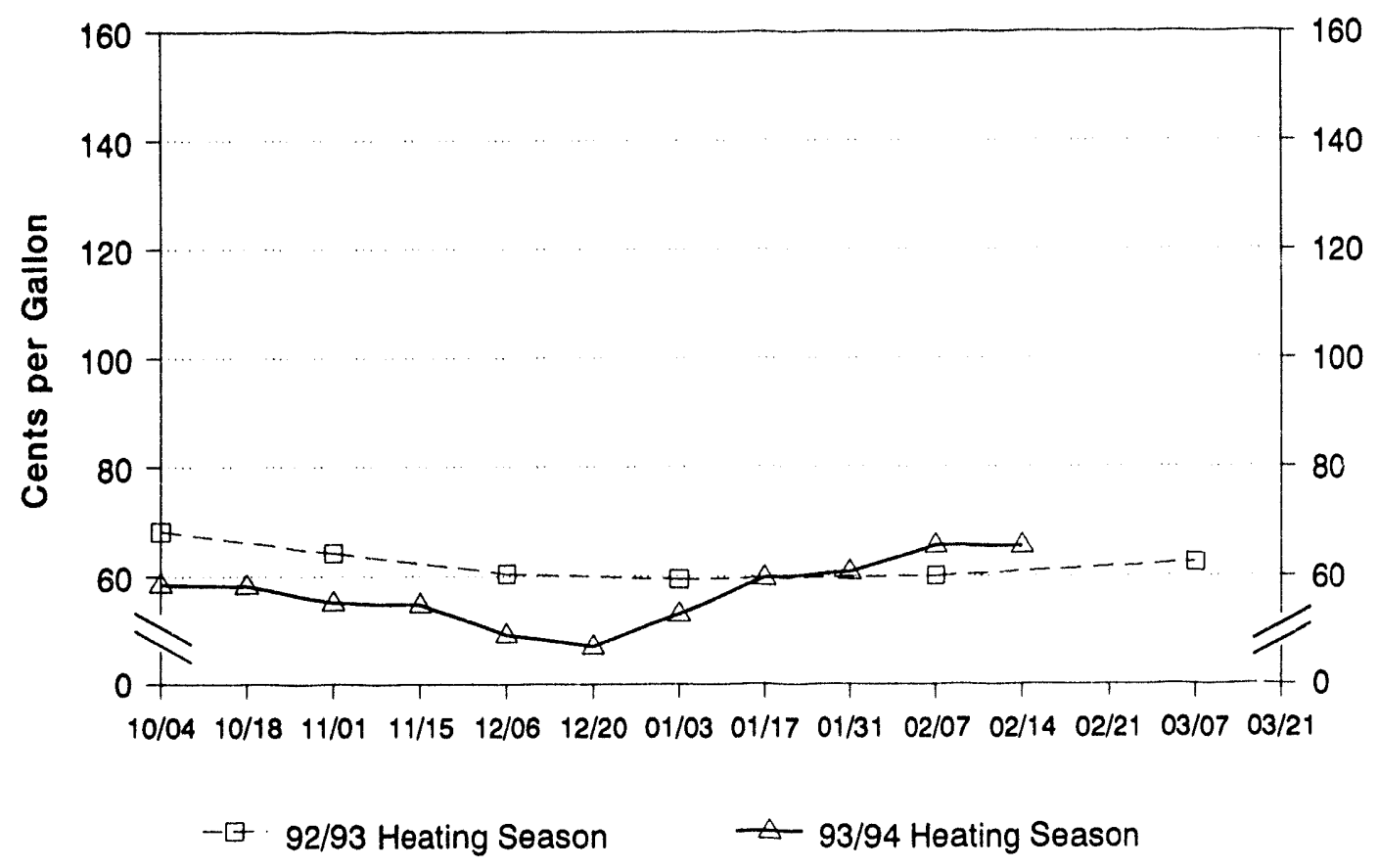

Source: Based on terminal quotes collected by the Computer Petroleum Corporation, Inc.

Figure 28. Wholesale Heating Oil Prices, Central Atlantic

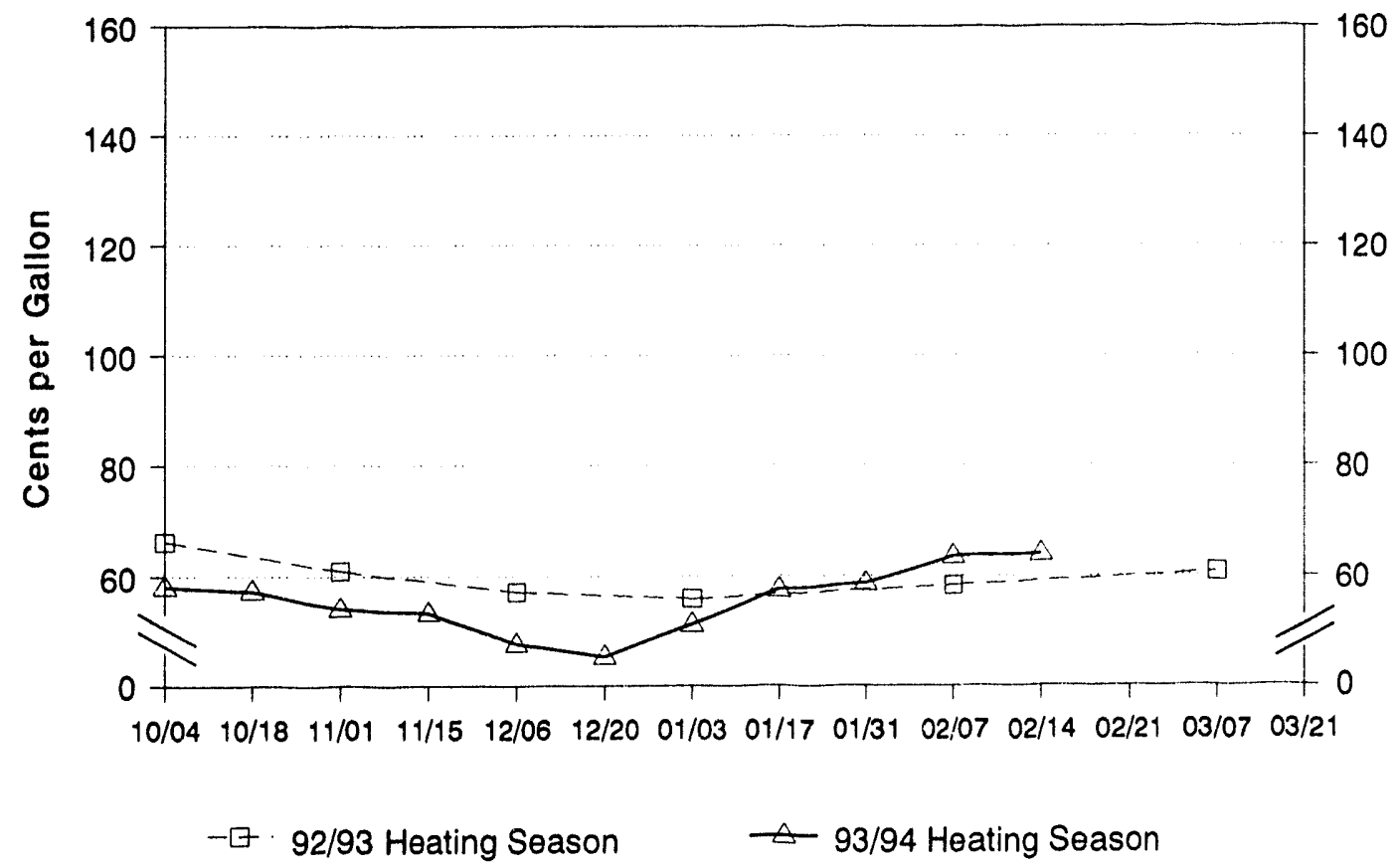

Source: Based on terminal quotes collected by the Computer Petroleum Corporation, Inc. 
Figure 29. Wholesale Heating Oll Prices, Lower Atlantic

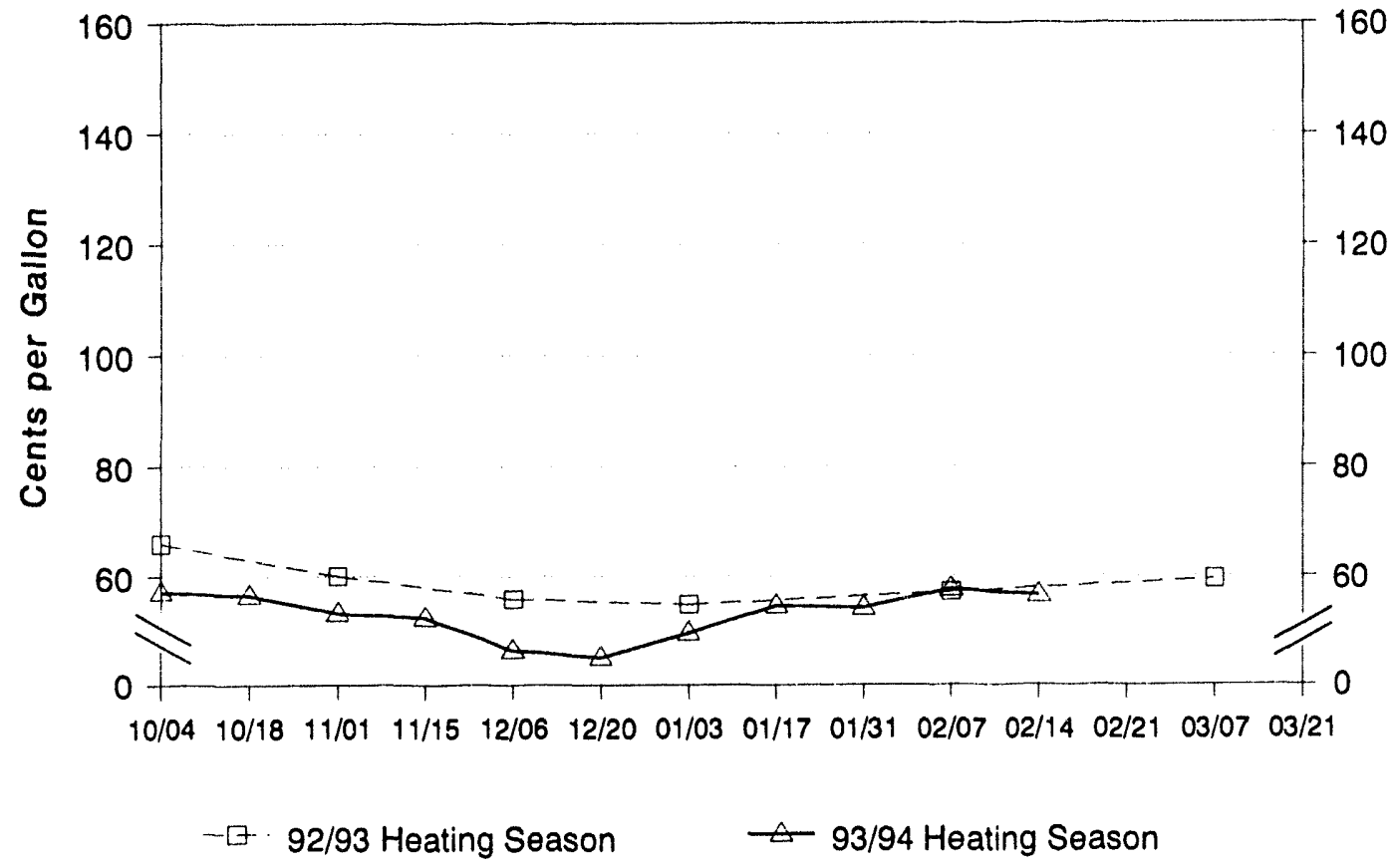

Source: Based on terminal quotes collected by the Computer Petroleum Corporation, Inc.

Figure 30. Wholesale Heating Oil Prices, Midwest

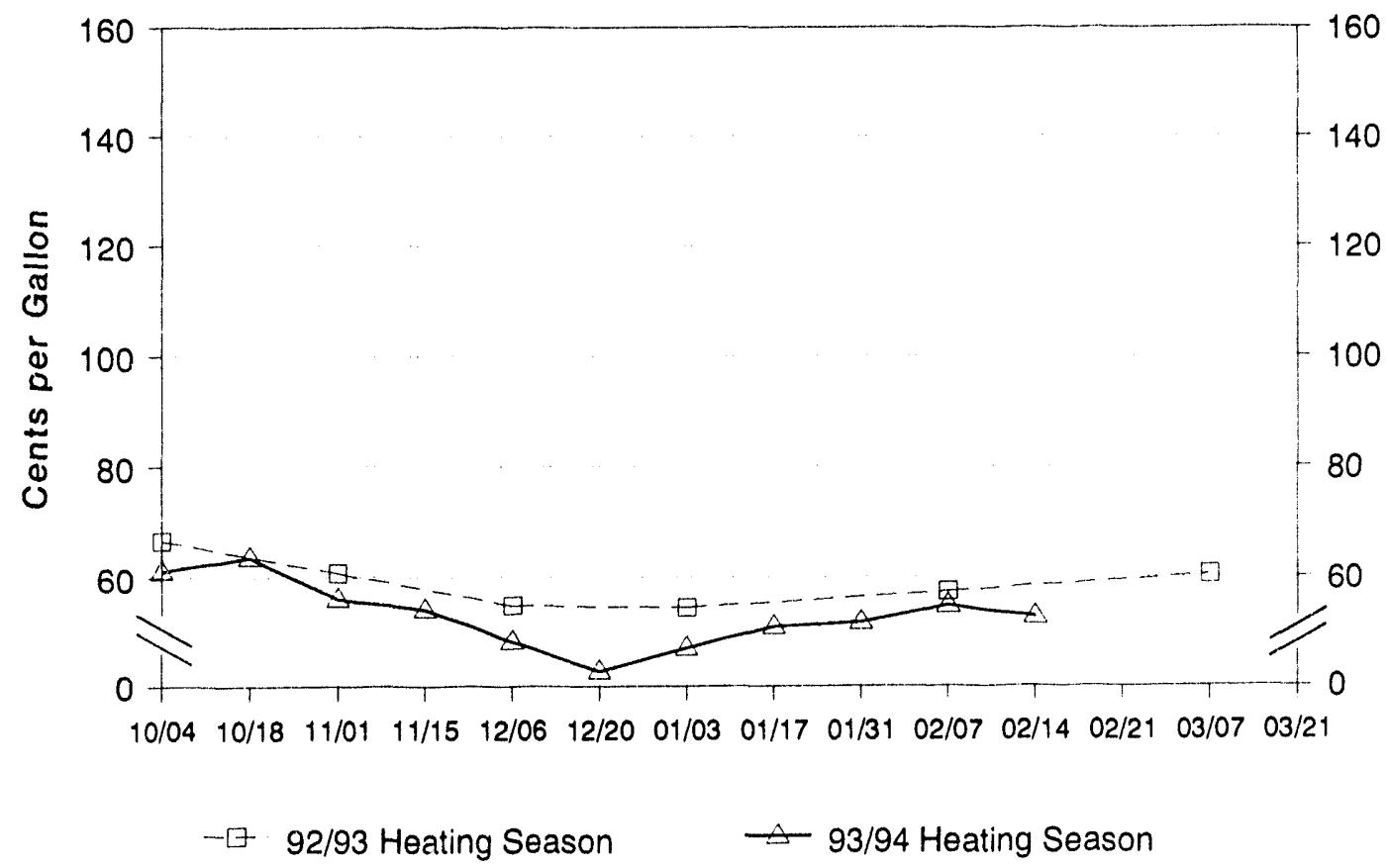

Source: Based on terminal quotes collected by the Computer Petroleum Corporation, Inc. 
Table 10. Wholesale Propane Prices by Reglon and State (Cents per Gallon)

\begin{tabular}{|c|c|c|c|c|c|c|}
\hline \multirow[b]{2}{*}{ Region/State } & \multicolumn{6}{|c|}{ 1992/93 Heating Season } \\
\hline & October & November & December & January & February & March \\
\hline Average & 38.9 & 38,8 & 39.7 & 48.5 & 39.2 & 47.1 \\
\hline East Coast (PADD) & 45.1 & 42.6 & 39.7 & 421 & 40.8 & 42.8 \\
\hline Central Atlantic (PADD IY) & 45.3 & 42.9 & 40.0 & 42.8 & 41.6 & 43.9 \\
\hline Lower Atlantic (PADD IZ) & 45.0 & 42.2 & 39.1 & 41.2 & 39.4 & 41.2 \\
\hline Mowest (PADD II) & 37.6 & 37.8 & 39.6 & 50.0 & 38.8 & 48.1 \\
\hline
\end{tabular}

\begin{tabular}{|c|c|c|c|c|c|c|c|c|c|c|c|c|}
\hline \multirow[b]{2}{*}{ Reglon/State } & \multicolumn{12}{|c|}{ 1993/94 Heating Season } \\
\hline & $10 / 04$ & $10 / 18$ & $11 / 01$ & $11 / 15$ & $12 / 06$ & $12 / 20$ & $01 / 03$ & $01 / 17$ & $01 / 31$ & $02 / 07$ & $02 / 14$ & $02 / 21$ \\
\hline Averago & 38.2 & 38.2 & 36.3 & 35.9 & 32,5 & 30.2 & 31,7 & 32.7 & 33.2 & 36.5 & 35.5 & \\
\hline East Coast (PADO ) & 37.9 & 381 & 36.5 & 36,4 & 34,3 & 32,9 & 34,9 & 37,1 & 39.4 & 43.3 & 43.0 & \\
\hline oentral Atlantlo (PADD IM) & 38,6 & 387 & 36.9 & 371 & 349 & 33.6 & 34.7 & 36.3 & 38.1 & 42.4 & 41.7 & \\
\hline New York & 38.9 & 38.9 & 37.1 & 37.3 & 35.2 & 33.9 & 35.0 & 36.5 & 38.3 & 42.5 & 41.8 & \\
\hline Pennsylvania & 38.3 & 38.5 & 36.8 & 36.9 & 34.7 & 33.4 & 34.4 & 36.2 & 38.0 & 42.3 & 41.6 & \\
\hline Lower Atlantic (PADD (Z) & 36.9 & 37.2 & 35.8 & 35.5 & 33.4 & 318 & 35,2 & 38.3 & 41.3 & 44.7 & 44,9 & \\
\hline North Carolina & 36.9 & 37.2 & 35.8 & 35.5 & 33.4 & 31.8 & 35.2 & 38.3 & 41.3 & 44.7 & 44.9 & \\
\hline Midwest (PADD II) & 38.3 & 38.3 & 36.3 & 35.8 & 32, & 29.6 & 30.9 & 31,7 & 317 & 34.9 & 33.7 & \\
\hline Illinois & 39.9 & 39.8 & 37.9 & 37.3 & 33.6 & 30.6 & 32.3 & 32.6 & 33.6 & 36.2 & 34.8 & \\
\hline Indiana & 36.8 & 36.8 & 35.3 & 34.8 & 32.8 & 31.4 & 32.7 & 34.0 & 35.2 & 39.4 & 38.3 & \\
\hline lowa & 39.0 & 39.1 & 37.0 & 36.3 & 31.9 & 29.3 & 30.2 & 31.0 & 30.2 & 33.2 & 32.1 & \\
\hline Kansas & 36.3 & 36.2 & 33.9 & 33.5 & 28.8 & 26.4 & 27.7 & 28.4 & 27.4 & 31.0 & 29.0 & \\
\hline Minnesota & 39.2 & 39.4 & 37.3 & 36.7 & 32.7 & 29.9 & 30.6 & 31.2 & 30.9 & 33.5 & 32.5 & \\
\hline Missouri & 38.6 & 38.5 & 36.4 & 35.8 & 32.2 & 29.5 & 30.6 & 31.5 & 30.8 & 33.8 & 32.5 & \\
\hline North Dakota & 38.4 & 38.5 & 35.6 & 35.8 & 34.1 & 31.5 & 31.8 & 32.7 & 33.2 & 33.8 & 33.5 & \\
\hline Ohio & 37.0 & 36.9 & 35.3 & 35.0 & 32.9 & 31.4 & 33.0 & 33.9 & 35.9 & 39.5 & 38.7 & \\
\hline South Dakota & 39.6 & 39.7 & 37.1 & 36.9 & 32.1 & 29.4 & 30.5 & 31.4 & 30.4 & 3.7 .3 & 32.0 & \\
\hline Wisconsin & 41.3 & 41.7 & 40.0 & 40.0 & 35.3 & 31.9 & 34.4 & 35.8 & 35.8 & 39.5 & 39.5 & \\
\hline
\end{tabular}

P=Preliminary data.

Source: These data are average prices collected by the Computer Petroleum Corporation, Inc. 
Figure 31. Wholesale Propane Prices, Central Atlantic

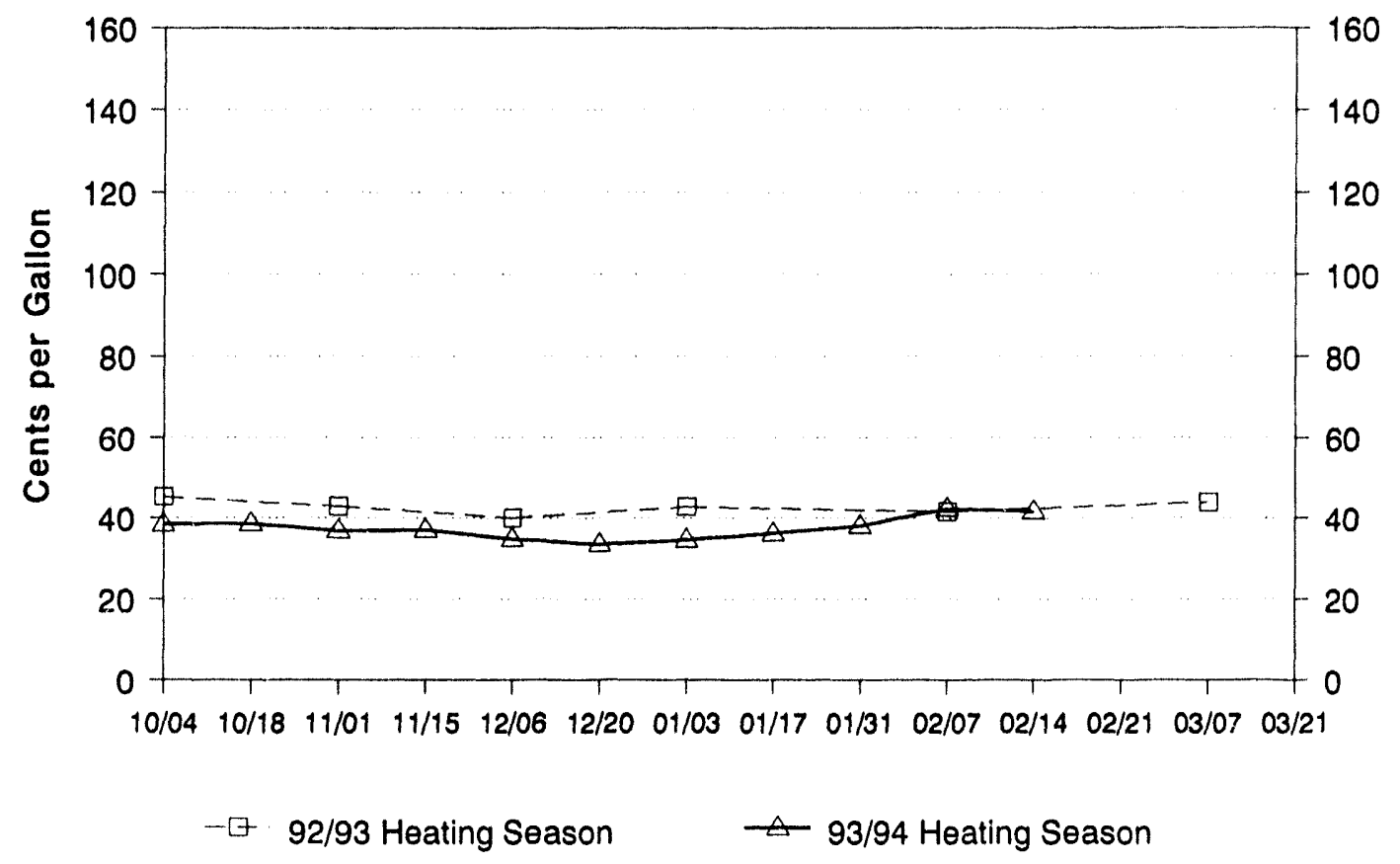

Source: Based on data collected by the Computer Petroleum Corporation, Inc.

Figure 32. Wholesale Propane Prices, Lower Atlantic

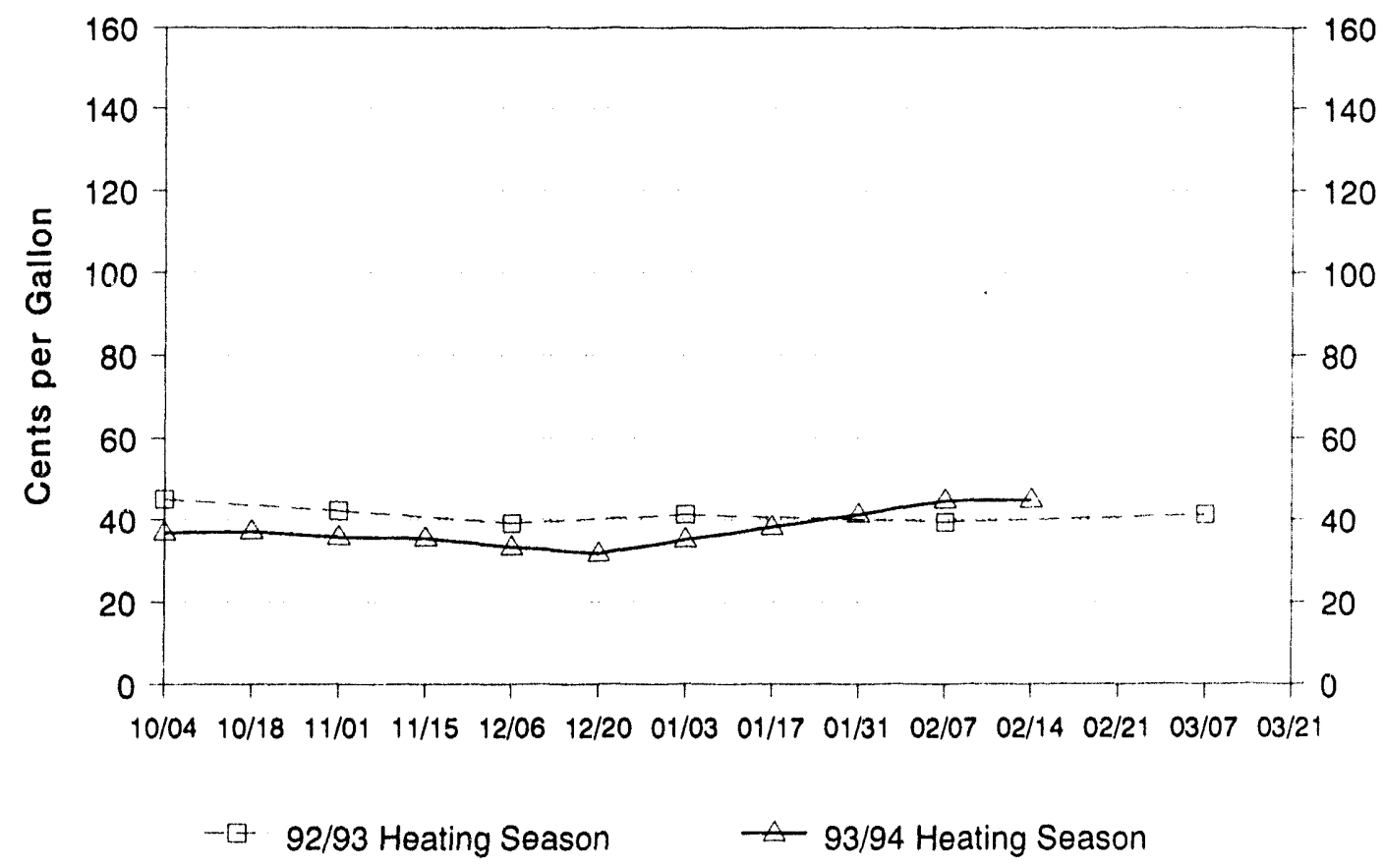

Source: Based on data collected by the Computer Petroleum Corporation, Inc. 
Figure 33. Wholesale Propane Prices, Midwest

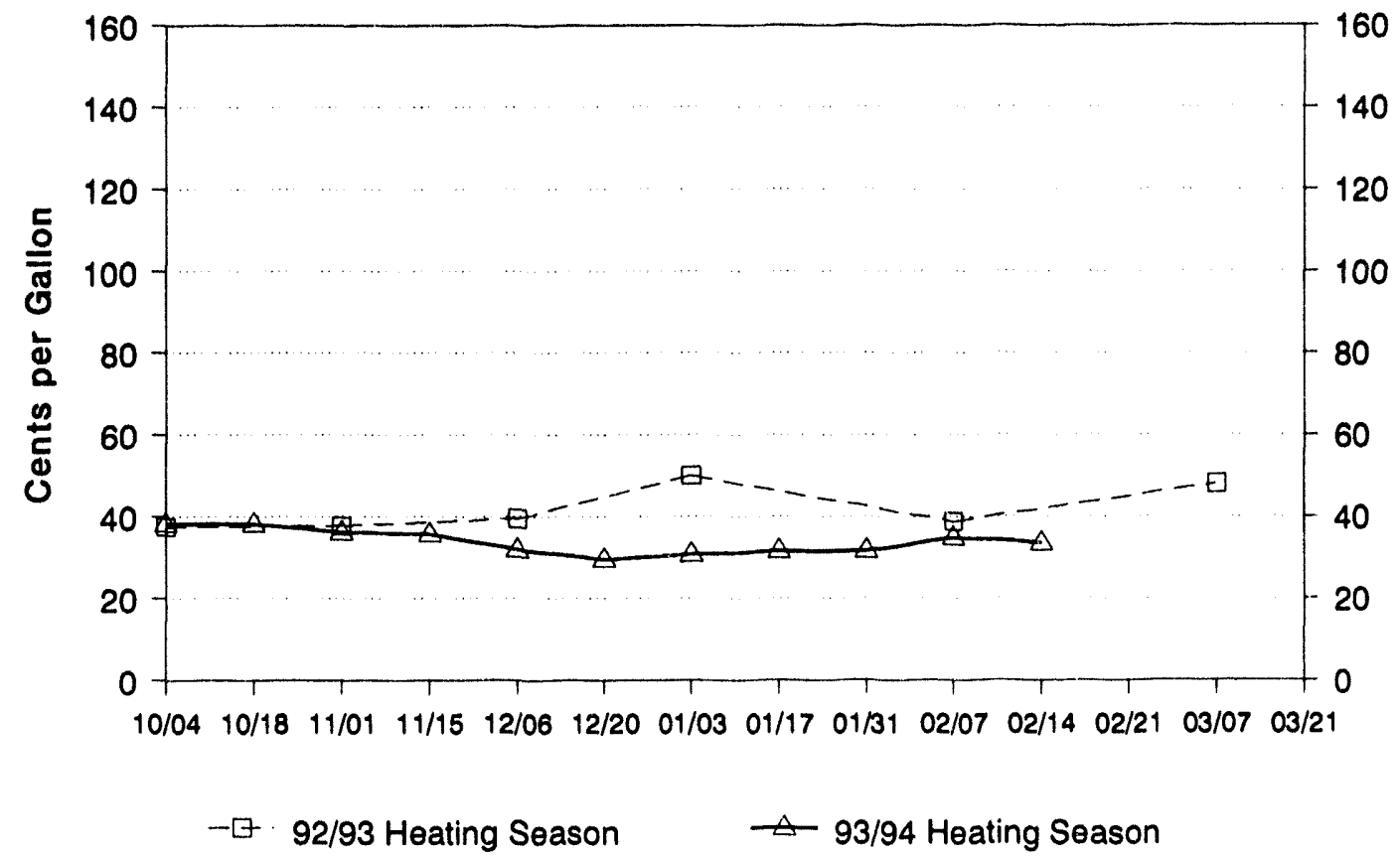

Source: Based on data collected by the Computer Petroleum Corporation, Inc. 
Table 11. U.S. Crude Oll and Petroleum Product Prices

(Cents per Gallon, Except Where Noted)

\begin{tabular}{|c|c|c|c|c|c|c|c|c|}
\hline \multirow[b]{2}{*}{$\begin{array}{l}\text { Report } \\
\text { Perlod }\end{array}$} & \multirow{2}{*}{$\begin{array}{c}\text { Crude } \\
\text { WTI } \\
\text { (Dollars per } \\
\text { Barrel) }\end{array}$} & \multicolumn{4}{|c|}{ No. 2 Distlllate } & \multicolumn{3}{|c|}{ Propane } \\
\hline & & Spot & Terminal & $\begin{array}{c}\text { Resl- } \\
\text { dentlal }\end{array}$ & $\begin{array}{l}\text { Dlesel } \\
\text { Retall }\end{array}$ & Spot & Terminal & $\begin{array}{l}\text { Resl- } \\
\text { dential }\end{array}$ \\
\hline \multicolumn{9}{|l|}{ Monthly } \\
\hline $02 / 93$ & 20.08 & 55.9 & 60.0 & 97.6 & 122.0 & 33.0 & 38.9 & 94.6 \\
\hline $03 / 93$ & 20.32 & 58.0 & 62.2 & 98.1 & 122.0 & 34.2 & 42.1 & 95.6 \\
\hline $04 / 93$ & 20.25 & 55.5 & 59.9 & 93.2 & 122.4 & 34.3 & 38.2 & 93.0 \\
\hline $05 / 93$ & 19.95 & 54.5 & 58.5 & NA & 122.8 & 32.8 & 36.4 & NA \\
\hline $06 / 93$ & 19.09 & 52.5 & 56.7 & NA & 122.3 & 32.8 & 36.9 & NA \\
\hline $07 / 93$ & 17.87 & 49.9 & 54.0 & NA & 119.6 & 31.4 & 36.2 & NA \\
\hline $08 / 93$ & 18.01 & 51.0 & 55.4 & NA & 118.4 & 30.4 & 36.4 & NA \\
\hline $09 / 93$ & 17.51 & 52.0 & 56.4 & NA & 118.6 & 29.9 & 37.6 & NA \\
\hline $10 / 93$ & 18.12 & 54.0 & 58.4 & 94.2 & 128.9 & 29.3 & 37.6 & 87.1 \\
\hline $11 / 93$ & 16.61 & 50.2 & 54.5 & 94.7 & 127.5 & 27.5 & 36.1 & 87.8 \\
\hline $12 / 93$ & 14.51 & 43.5 & 48.0 & 93.2 & 123.5 & 24.5 & 31.4 & 88.1 \\
\hline $01 / 94$ & 15.03 & 50.4 & 56.3 & 93.4 & NA & 26.4 & 32.4 & 88.6 \\
\hline \multicolumn{9}{|l|}{ Week Ending } \\
\hline 12/23/93 & 14.40 & 43.0 & 46.9 & 92.6 & NA & 24.9 & 30.5 & 88.0 \\
\hline $12 / 31 / 93$ & 14.21 & 44.4 & 48.7 & NA & NA & 25.3 & 31.1 & NA \\
\hline $01 / 07 / 94$ & 15.03 & 48.1 & 51.1 & 92.1 & NA & 25.6 & 31.4 & 88.8 \\
\hline $01 / 14 / 94$ & 14.66 & 49.6 & 53.6 & NA & NA & 26.0 & 31.8 & NA \\
\hline $01 / 21 / 94$ & 15.02 & 50.1 & 58.3 & 94.7 & NA & 26.7 & 32.8 & 88.4 \\
\hline $01 / 28 / 94$ & 15.34 & 52.1 & 59.6 & NA & NA & 26.8 & 33.1 & NA \\
\hline $02 / 04 / 94$ & 15.76 & 58.0 & 64.4 & NA & NA & 30.0 & 35.3 & NA \\
\hline $02 / 11 / 94$ & 14.82 & 61.5 & 67.2 & NA & NA & 29.3 & 35.9 & NA \\
\hline $02 / 18 / 94$ & 14.14 & 51.7 & 64.5 & 99.5 & NA & 27.8 & 35.0 & 90.9 \\
\hline \multicolumn{9}{|l|}{ Dally } \\
\hline $01 / 28 / 94$ & 15.37 & 53.2 & 61.6 & NA & NA & 27.4 & 33.2 & NA \\
\hline $01 / 31 / 94$ & 15.24 & 54.2 & 61.8 & NA & NA & 28.6 & 33.5 & NA \\
\hline $02 / 01 / 94$ & 15.91 & 56.8 & 62.0 & NA & NA & 29.9 & 34.1 & NA \\
\hline $02 / 02 / 94$ & 16.06 & 59.4 & 63.7 & NA & NA & 31.6 & 34.8 & NA \\
\hline $02 / 03 / 94$ & 15.97 & 60.7 & 66.3 & NA & NA & NA & 36.7 & NA \\
\hline $02 / 04 / 94$ & 15.63 & 58.8 & 68.0 & NA & NA & 29.8 & 37.3 & NA \\
\hline $02 / 08 / 94$ & 15.12 & 60.8 & 66.8 & NA & NA & 29.6 & 36.0 & NA \\
\hline $02 / 09 / 94$ & 14.64 & 60.9 & 67.0 & NA & NA & 29.1 & 36.0 & NA \\
\hline $02 / 11 / 94$ & 14.71 & 62.8 & 67.7 & NA & NA & 29.1 & 35.6 & NA \\
\hline $02 / 14 / 94$ & 14.15 & 57.3 & 67.7 & 99.5 & NA & 28.8 & 35.6 & 90.9 \\
\hline $02 / 15 / 94$ & 14.13 & 53.4 & 65.9 & NA & NA & 27.9 & 35.3 & NA \\
\hline $02 / 16 / 94$ & 13.89 & 51.0 & 64.9 & NA & NA & 27.6 & 34.9 & NA \\
\hline $02 / 17 / 94$ & 14.26 & 48.5 & 62.6 & NA & NA & 27.4 & 34.7 & NA \\
\hline $02 / 18 / 94$ & 14.26 & 48.5 & 61.4 & NA & NA & 27.4 & 34.7 & NA \\
\hline
\end{tabular}

NA=Not available.

Source: - Spot West Texas Intermediate (WTI) at Cushing, Oklahoma; No. 2 distillate in New York Harbor from Reuters. Computer Petroleum Corp. rack (terminal) prices. - Residential No. 2 distillate and propane prices from State Heating Oil and Propane Program. Diesel Retail prices from Lundberg PS. - Mt. Belvieu, Texas, spot propane prices from Platts' Oilgram Price Report. 
Table 12. Petroleum Product Prices for Selected Citles (Cents per Gallon)

\begin{tabular}{|c|c|c|c|c|c|c|}
\hline \multirow{3}{*}{$\begin{array}{l}\text { Report } \\
\text { Perlod }\end{array}$} & \multicolumn{3}{|c|}{ Chlcago } & \multicolumn{3}{|c|}{ Houston } \\
\hline & \multicolumn{2}{|c|}{ No. 2 Dlatillate } & \multirow{2}{*}{$\begin{array}{l}\text { Propane } \\
\text { Terminal }\end{array}$} & \multicolumn{2}{|c|}{ No. 2 Diatlllats } & \multirow{2}{*}{$\begin{array}{l}\text { Propane } \\
\text { Terminal }\end{array}$} \\
\hline & Spot & Terminal & & Spot & TermInal & \\
\hline \multicolumn{7}{|l|}{ Monthly } \\
\hline $\begin{array}{l}02 / 93 \\
03 / 93 \\
04 / 93 \\
05 / 93 \\
06 / 93 \\
07 / 93 \\
08 / 93 \\
09 / 93 \\
10 / 93 \\
11 / 93 \\
12 / 93 \\
01 / 94\end{array}$ & $\begin{array}{l}54.2 \\
57.0 \\
55.5 \\
55.4 \\
53.4 \\
47.4 \\
46.2 \\
50.8 \\
51.4 \\
46.7 \\
39.6 \\
43.4\end{array}$ & $\begin{array}{l}56.7 \\
59.5 \\
57.3 \\
57.1 \\
55.2 \\
49.4 \\
47.8 \\
52.2 \\
59.0 \\
50.8 \\
42.7 \\
46.6\end{array}$ & $\begin{array}{l}40.7 \\
44.9 \\
38.5 \\
37.1 \\
38.6 \\
38.0 \\
38.5 \\
39.7 \\
39.7 \\
37.9 \\
32.1 \\
32.2\end{array}$ & $\begin{array}{l}53.3 \\
55.7 \\
53.5 \\
53.4 \\
50.8 \\
48.1 \\
50.4 \\
49.9 \\
51.6 \\
48.2 \\
41.0 \\
45.4\end{array}$ & $\begin{array}{l}55.5 \\
57.3 \\
55.5 \\
55.8 \\
53.5 \\
50.1 \\
51.7 \\
52.0 \\
55.6 \\
52.4 \\
44.0 \\
49.1\end{array}$ & $\begin{array}{l}35.2 \\
36.7 \\
37.0 \\
35.2 \\
34.5 \\
34.0 \\
33.1 \\
32.8 \\
32.0 \\
30.5 \\
27.8 \\
29.7\end{array}$ \\
\hline \multicolumn{7}{|l|}{ Week Endling } \\
\hline $\begin{array}{l}12 / 23 / 93 \\
12 / 31 / 93 \\
01 / 07 / 94 \\
01 / 14 / 94 \\
01 / 21 / 94 \\
01 / 28 / 94 \\
02 / 04 / 94 \\
02 / 11 / 94 \\
02 / 18 / 94\end{array}$ & $\begin{array}{l}38.5 \\
38.9 \\
41.2 \\
41.7 \\
43.4 \\
46.2 \\
51.5 \\
47.8 \\
43.0\end{array}$ & $\begin{array}{l}40.8 \\
42.4 \\
43.3 \\
45.1 \\
47.9 \\
48.6 \\
52.2 \\
51.6 \\
57.0\end{array}$ & $\begin{array}{l}30.9 \\
31.5 \\
31.9 \\
31.8 \\
32.6 \\
32.2 \\
34.3 \\
34.7 \\
33.6\end{array}$ & $\begin{array}{l}40.9 \\
41.1 \\
44.6 \\
45.0 \\
45.6 \\
45.7 \\
49.3 \\
47.4 \\
42.9\end{array}$ & $\begin{array}{l}43.5 \\
44.0 \\
45.8 \\
48.0 \\
50.7 \\
50.2 \\
53.4 \\
54.2 \\
49.8\end{array}$ & $\begin{array}{l}27.0 \\
28.1 \\
27.9 \\
29.0 \\
29.6 \\
31.4 \\
33.8 \\
34.9 \\
33.8\end{array}$ \\
\hline \multicolumn{7}{|l|}{ Dally } \\
\hline $\begin{array}{l}01 / 28 / 94 \\
01 / 31 / 94 \\
02 / 01 / 94 \\
02 / 02 / 94 \\
02 / 03 / 94 \\
02 / 04 / 94 \\
02 / 08 / 94 \\
02 / 09 / 94 \\
02 / 11 / 94 \\
02 / 14 / 94 \\
02 / 15 / 94 \\
02 / 16 / 94 \\
02 / 17 / 94 \\
02 / 18 / 94\end{array}$ & $\begin{array}{l}47.2 \\
47.2 \\
50.6 \\
53.6 \\
53.6 \\
52.6 \\
47.8 \\
47.8 \\
48.0 \\
44.3 \\
44.3 \\
41.5 \\
42.5 \\
42.8\end{array}$ & $\begin{array}{l}50.2 \\
50.1 \\
50.3 \\
52.0 \\
54.3 \\
54.3 \\
52.0 \\
51.9 \\
50.9 \\
50.9 \\
48.8 \\
47.5 \\
46.1 \\
45.9\end{array}$ & $\begin{array}{l}32.4 \\
32.6 \\
32.7 \\
34.1 \\
35.8 \\
36.4 \\
34.7 \\
34.9 \\
34.5 \\
34.5 \\
34.2 \\
33.2 \\
33.1 \\
33.2\end{array}$ & $\begin{array}{l}46.8 \\
46.8 \\
49.1 \\
50.5 \\
50.5 \\
49.3 \\
47.4 \\
47.4 \\
47.2 \\
43.9 \\
43.9 \\
41.8 \\
42.3 \\
42.8\end{array}$ & $\begin{array}{l}51.8 \\
51.7 \\
51.4 \\
52.7 \\
55.1 \\
56.0 \\
54.6 \\
54.6 \\
53.3 \\
53.3 \\
51.2 \\
50.0 \\
47.9 \\
46.8\end{array}$ & $\begin{array}{l}31.7 \\
32.2 \\
32.5 \\
33.0 \\
35.5 \\
35.6 \\
35.0 \\
34.9 \\
34.9 \\
34.9 \\
34.0 \\
33.5 \\
33.3 \\
33.3\end{array}$ \\
\hline
\end{tabular}

See footnotes at end of table. 
Table 12. Petroleum Product Prices for Selected Cities (Continued) (Cents per Gallon)

\begin{tabular}{|c|c|c|c|c|c|c|}
\hline \multirow{3}{*}{$\begin{array}{l}\text { Report } \\
\text { Perlod }\end{array}$} & \multicolumn{3}{|c|}{ Los Angeles } & \multicolumn{3}{|c|}{ New York } \\
\hline & \multicolumn{2}{|c|}{ No. 2 Distillate } & \multirow{2}{*}{$\begin{array}{l}\text { Propane } \\
\text { Terminal }\end{array}$} & \multicolumn{2}{|c|}{ No. 2 Dlstlllate } & \multirow{2}{*}{$\begin{array}{l}\text { Propene } \\
\text { Terminal }\end{array}$} \\
\hline & Spot & Terminal & & Spot & TermInal & \\
\hline \multicolumn{7}{|l|}{ Monthly } \\
\hline $02 / 93$ & 55.4 & 60.6 & 44.9 & 55.9 & 60.0 & 42.9 \\
\hline $03 / 93$ & 58.5 & 62.1 & 41.0 & 58.0 & 62.2 & 45.4 \\
\hline $04 / 93$ & 59.7 & 63.6 & 38.7 & 55.5 & 59.9 & 44.7 \\
\hline $05 / 93$ & 58.7 & 63.7 & 35.9 & 54.5 & 58.5 & 42.5 \\
\hline $06 / 93$ & 56.9 & 60.2 & 33.0 & 52.5 & 56.7 & 41.6 \\
\hline $07 / 93$ & 54.8 & 58.1 & 33.3 & 49.9 & 54.0 & 40.8 \\
\hline $08 / 93$ & 55.6 & 57.2 & 35.8 & 51.0 & 55.4 & 39.9 \\
\hline $09 / 93$ & 59.7 & 63.6 & 41.0 & 52.0 & 56.4 & 39.6 \\
\hline $10 / 93$ & 73.6 & 66.6 & 45.4 & 54.0 & 58.4 & 39.5 \\
\hline $11 / 93$ & 61.2 & NA & 46.0 & 50.2 & 54.5 & 37.9 \\
\hline $12 / 93$ & 48.5 & 46.3 & 46.0 & 43.5 & 48.0 & 35.3 \\
\hline $01 / 94$ & 50.6 & 47.0 & 48.5 & 50.4 & 56.3 & 37.2 \\
\hline \multicolumn{7}{|l|}{ Week Ending } \\
\hline $12 / 23 / 93$ & 49.1 & 45.5 & 46.0 & 43.0 & 46.9 & 34.8 \\
\hline $12 / 31 / 93$ & 49.1 & 46.3 & 46.0 & 44.4 & 48.7 & 35.3 \\
\hline $01 / 07 / 94$ & 50.2 & 46.7 & 48.7 & 48.1 & 51.1 & 35.6 \\
\hline $01 / 14 / 94$ & 49.9 & 47.0 & 49.0 & 49.6 & 53.6 & 36.2 \\
\hline $01 / 21 / 94$ & 50.0 & 47.0 & 49.0 & 50.1 & 58.3 & 37.6 \\
\hline $01 / 28 / 94$ & 52.1 & 47.2 & 47.9 & 52.1 & 59.6 & 38.5 \\
\hline $02 / 04 / 94$ & 52.0 & 49.0 & 46.0 & 58.0 & 64.4 & 41.3 \\
\hline $02 / 11 / 94$ & 53.0 & 51.2 & 44.0 & 61.5 & 67.2 & 42.7 \\
\hline $02 / 18 / 94$ & 53,3 & 51.8 & 43,2 & 517 & 64.5 & 422 \\
\hline \multicolumn{7}{|l|}{ Dally } \\
\hline $01 / 28 / 94$ & 52.5 & 47.5 & 46.0 & 53.2 & 61.6 & 38.9 \\
\hline $01 / 31 / 94$ & 53.0 & 48.0 & 46.0 & 54.2 & 61.8 & 39.2 \\
\hline $02 / 01 / 94$ & 52.0 & 48.0 & 46.0 & 56.8 & 62.0 & 39.8 \\
\hline $02 / 02 / 94$ & 52.5 & 49.0 & 46.0 & 59.4 & 63.7 & 40.2 \\
\hline $02 / 03 / 94$ & 53.0 & 50.0 & 46.0 & 60.7 & 66.3 & 43.6 \\
\hline $02 / 04 / 94$ & 49.5 & 50.0 & 46.0 & 58.8 & 68.0 & 43.6 \\
\hline $02 / 08 / 94$ & 53.0 & 51.0 & 44.0 & 60.8 & 66.8 & 42.7 \\
\hline $02 / 09 / 94$ & 52.8 & 51.0 & 44.0 & 60.9 & 67.0 & 42.7 \\
\hline $02 / 11 / 94$ & 53.3 & 51.5 & 44.0 & 62.8 & 67.7 & 42.7 \\
\hline $02 / 14 / 94$ & 55.0 & 51.5 & 44.0 & 57.3 & 67.7 & 42.7 \\
\hline $02 / 15 / 94$ & 54.3 & 51.5 & 43.0 & 53.4 & 65.9 & 42.3 \\
\hline $02 / 16 / 94$ & 52.3 & 51.5 & 45.0 & 51.0 & 64.9 & 42.3 \\
\hline $02 / 17 / 94$ & NA & 51.5 & 43.0 & 48.5 & 62.6 & 42.1 \\
\hline $02 / 18 / 94$ & 51.5 & 53.0 & 43.0 & 48.5 & 61,4 & 41.8 \\
\hline
\end{tabular}

NA=Not available.

Source: - No. 2 distillate spot prices in Chicago, Houston, and Los Angeles, are from Telerate; New York spot prices are from Reuters. $\cdot$ No. 2 distillate terminal

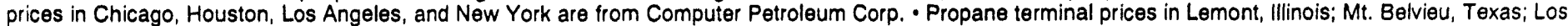
Angeles, California; and Selkirk, New York are from Computer Petroleum Corp. 


\section{Weather \\ Summary}

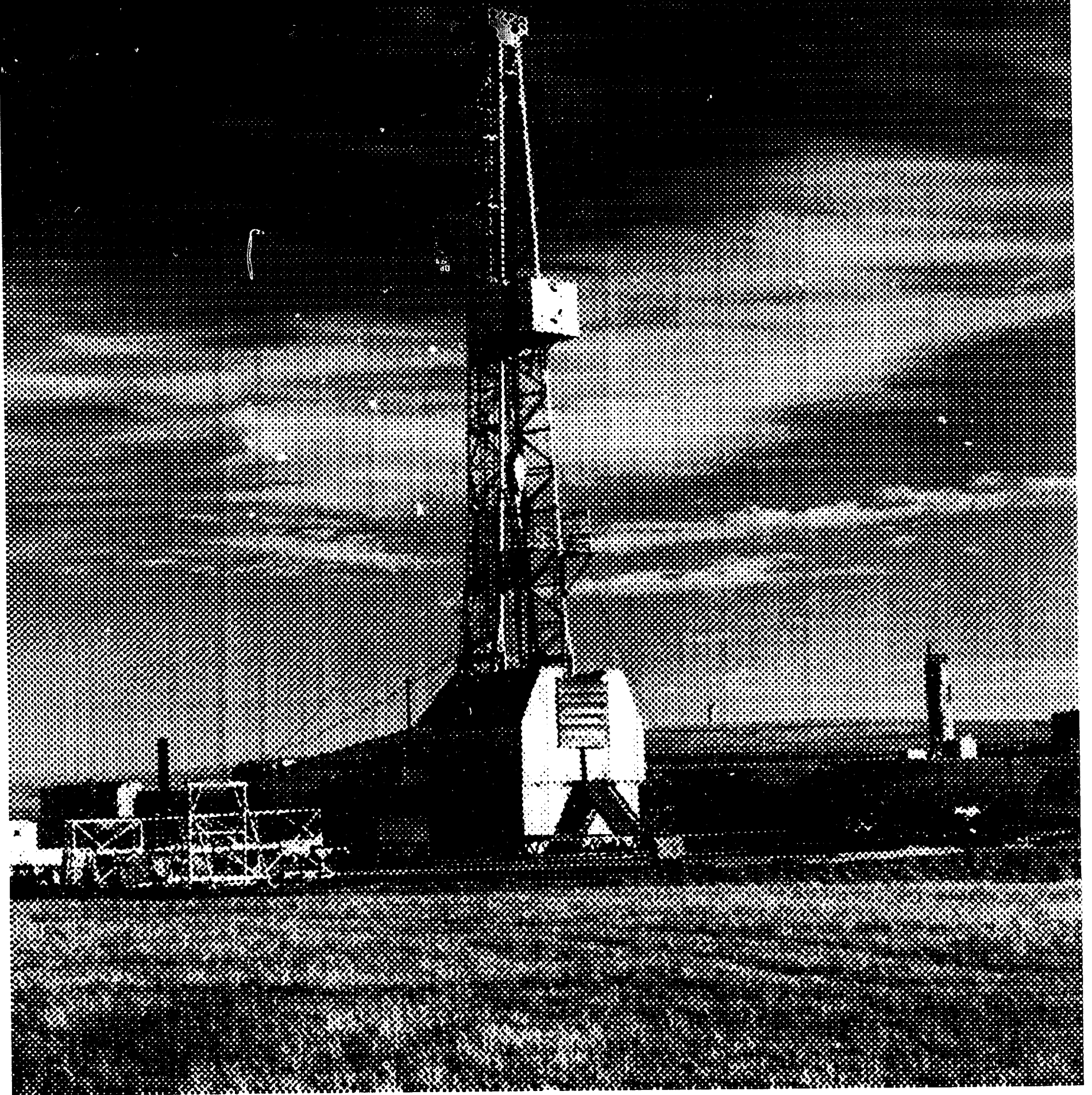




\section{United States Weather Summary}

\section{6-10 Day Outlook- February 27 Through March 3, 1994}

Near normal average temperatures are expected for nearly all of the southeastern two-thirds of California, the southern half of Nevada, northwestern Arizona, the upper Mississippi Valley, the western and southern areas of the Great Lakes region, the Ohio Valley, Virginia, the Carolinas, eastern Tennessee and Georgia, the eastern half of the Florida panhandle, and the Florida peninsula. Below normal temperatures are expected for the area in and around San Diego, New England and New York, New Jersey, Delaware, nearly all of Pennsylvania and Maryland, portions of northern Virginia, and the area around the Sault Sainte Marie area of Michigan. Above normal temperatures are indicated for most of the western and central regions of the Nation as well as unspecified areas.

Little or no precipitation is forecast for eastern Montana, the Dakotas, western Minnesota, Nebraska, Kansas, Colorado, the western halves of Oklahoma and Texas, New Mexico, Arizona, southern Nevada, and the southeastern sections of California. Below normal amounts are expected for eastern Wyoming and the adjacent parts of both extreme southeastern Montana and north-central Colorado, the middle and upper portions of the Mississippi River Valley, extreme southeastern New York and most of New England. Above normal amounts are predicted over the Pacific Northwest, the western portions of Montana and Wyoming, northwestern Colorado, the northwestern two-thirds of Nevada and California, the lower Mississippi Valley, extreme southeastern Oklahoma, the eastern third of Texas, and the eastern half of the Florida peninsula.

(Refer to Figures 34 and 35).

\section{Day Outlook - Mid-February Through Mid-March 1994}

There is at least a 55 percent chance for above normal temperatures from Utah and eastern Arizona eastward across Colorado, southern Kansas, the southern Great Plains, the Gulf Coast region, southern Missouri, Arkansas, the southern sections of the Ohio Valley and Tennessee and the Atlantic Coast states as far north as Maryland and Delaware. The likelihood for warmer temperatures rises to as high as 60 percent over southeastern New Mexico, much of Texas, Louisiana, and the southern region of Mississippi, Alabama, Georgia, South Carolina, and Floricla. There is at least a 55 percent chance for below normal temperatures over New England, the upper peninsula of Michigan, extreme northern Wisconsin, much of Minnesota, North Dakota, northern South Dakota, most of Montana, northern and western Washington, and the coastal regions of Oregon and extreme northwestern California. In unspecified areas the average temperature probabilities are not expected to depart significantly from climatological values.

(Refer to Figure 36).

\section{Day Outlook - February 1994 Through April 1994}

Specifies at least a 55 percent chance of below normal temperatures in the area extending from the southern Plains eastward through the Gulf States and Tennessee Valley to the southeastern region of the Nation and then moving northeastward through the middle Atlantic states and southeastern Midwest to New England. Within this area there is at least a 65 percent chance for colder temperatures in the Florida Panhandle, southern Georgia, and Alabama. There is a 55 percent chance for above normal temperatures along the West Coast, extending eastward from the Pacific Northwest to the Northern Rockies. Included in this area are southwestern Arizona, all of California, western Nevada, all of Oregon, Washington and Montana, and northern Idaho and Wyoming. Within this area the probabilities for relatively mild conditions exceed 65 percent along the immediate West Coast from near Los Angeles northward to the Canadian border.

(Refer to Figure 37).

Source: National Weather Service, National Oceanic and Atmospheric Administration, U.S. Department of Commerce. 
Figure 34. 6 - 10 Day Temperature Outlook for February 27 Through March 3, 1994

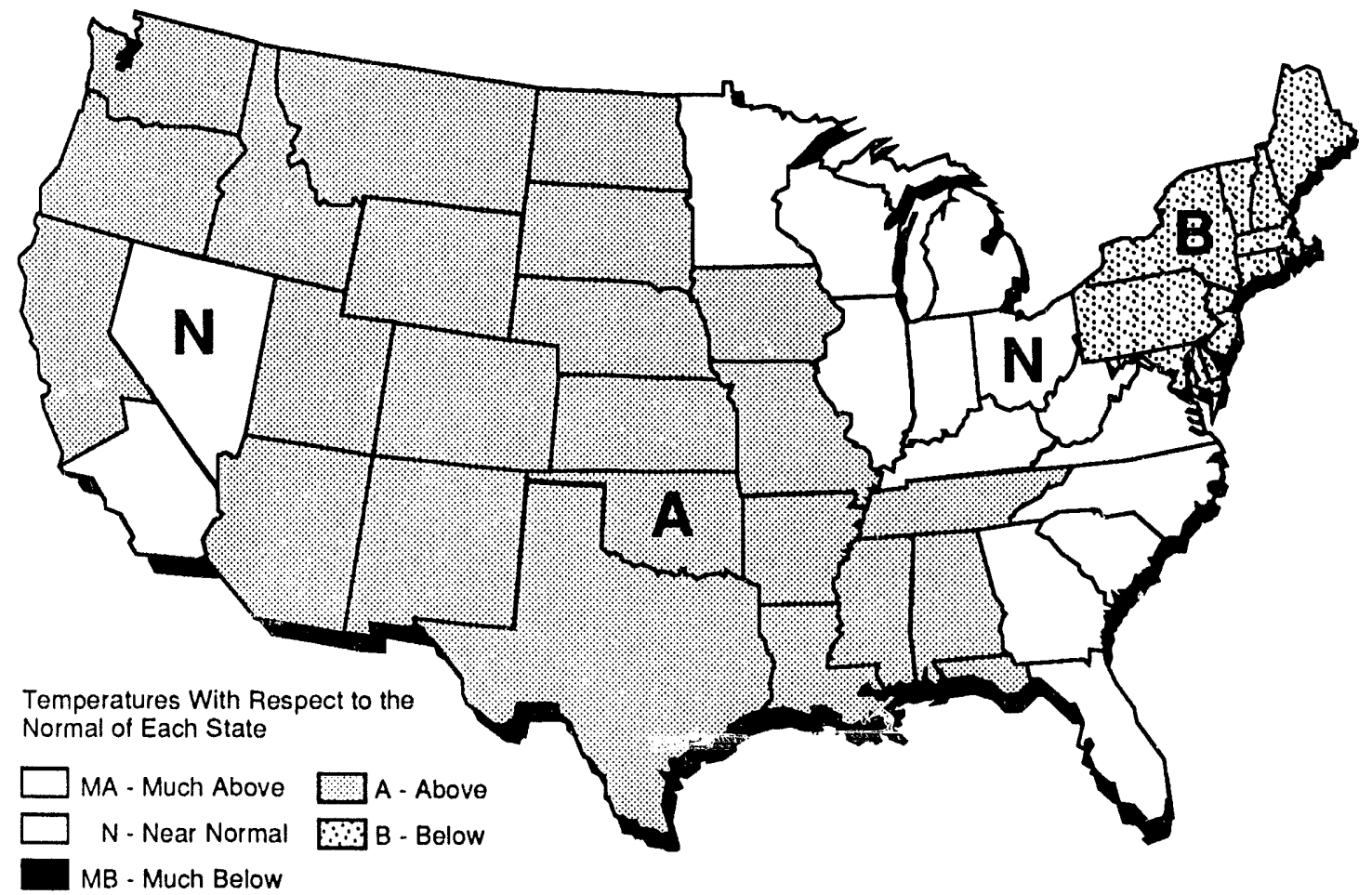

Source: National Weather Service, National Oceanic and Atmospheric Administration, U.S. Department of Commerce.

Figure 35. 6 - 10 Day Precipitation Outlook for February 27 Through March 3, 1994

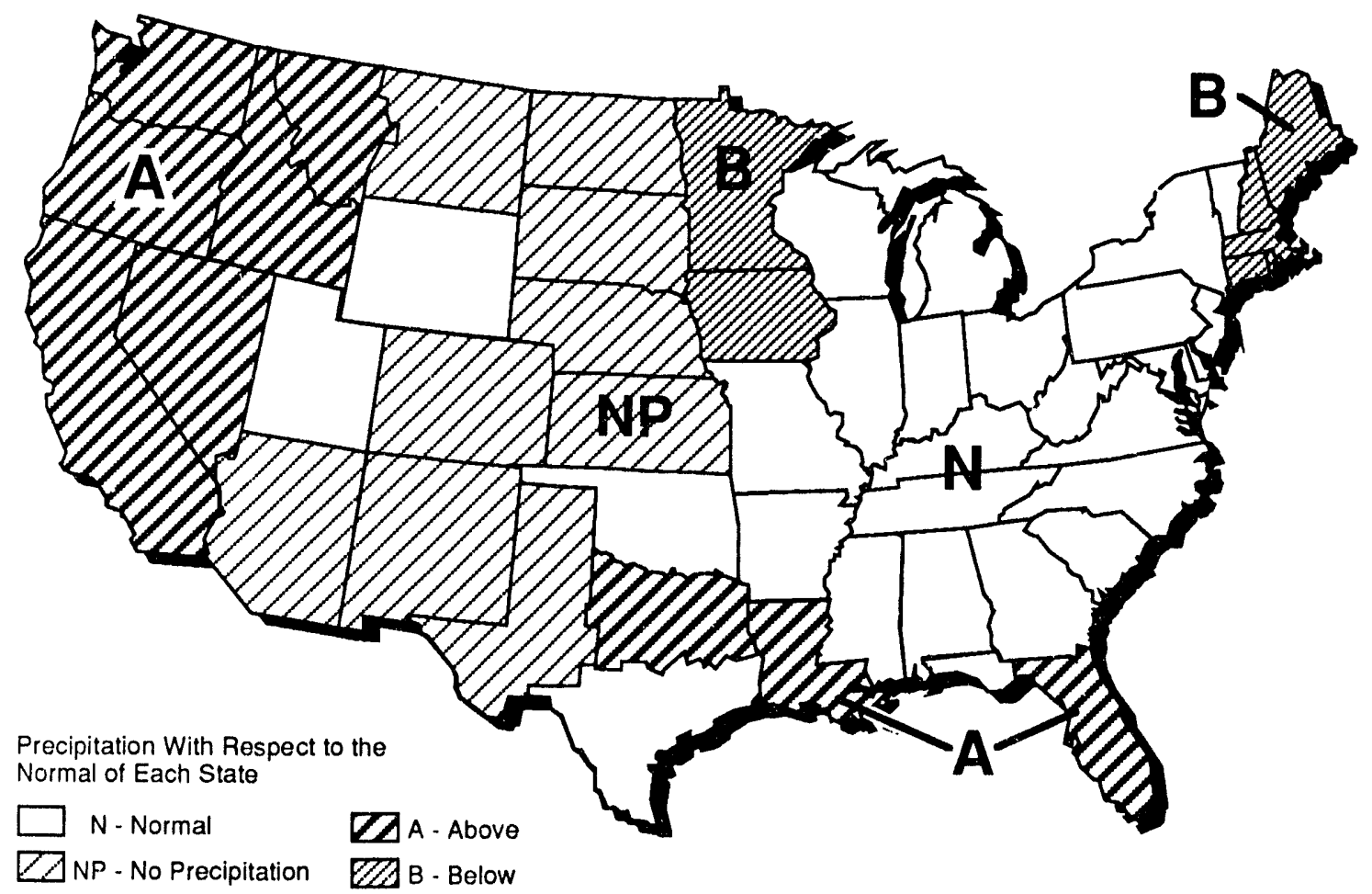

Source: National Weather Service, National Oceanic and Atmospheric Administration, U.S. Department of Commerce. 
Figure 36. 30 Day Temperature Outlook for Mid-February Through Mid-March 1994

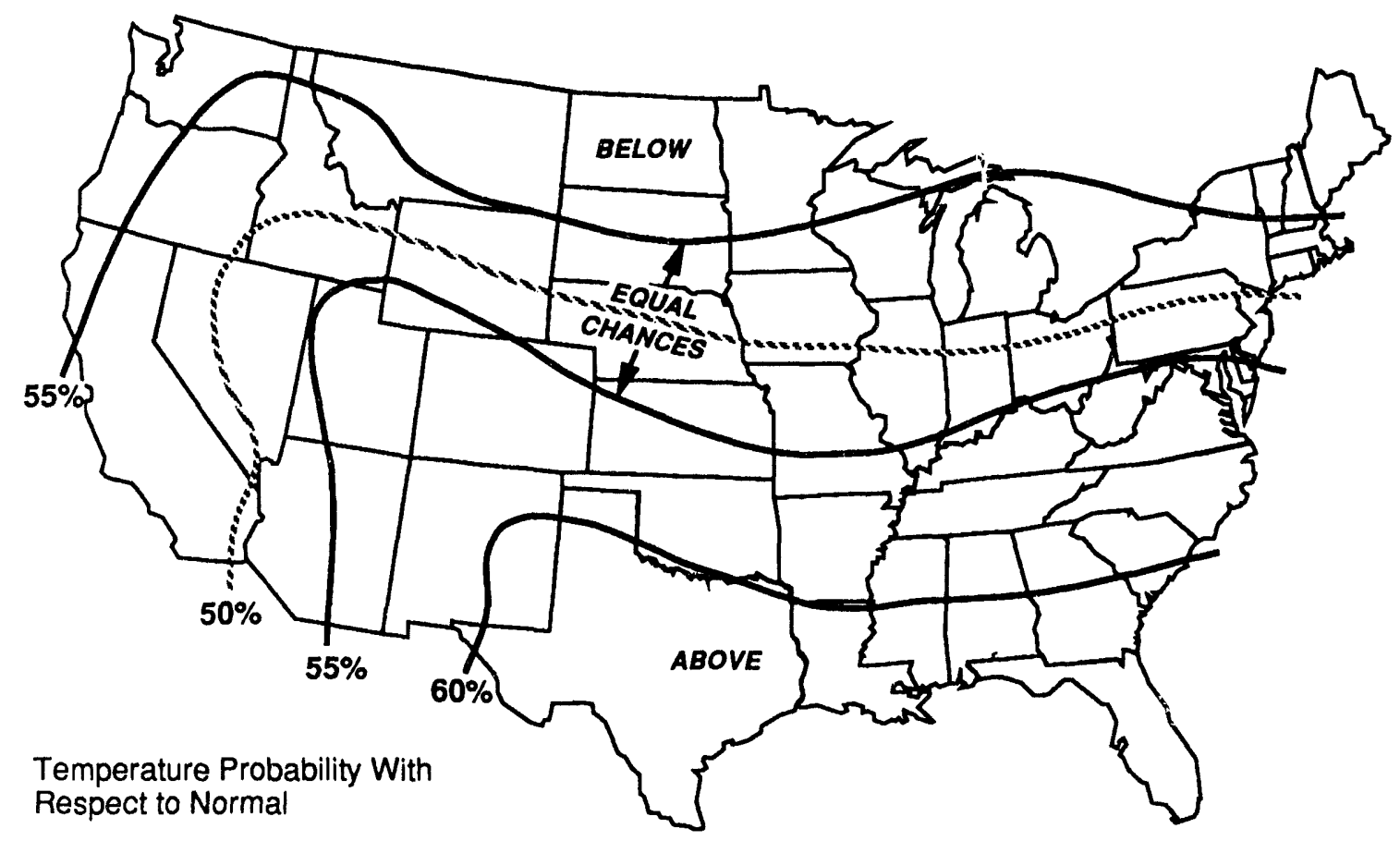

Source: National Weather Service, National Oceanic and Atmospheric Administration, U.S. Department of Commerce.

Figure 37. 90 Day Temperature Outlook for February 1994 Through April 1994

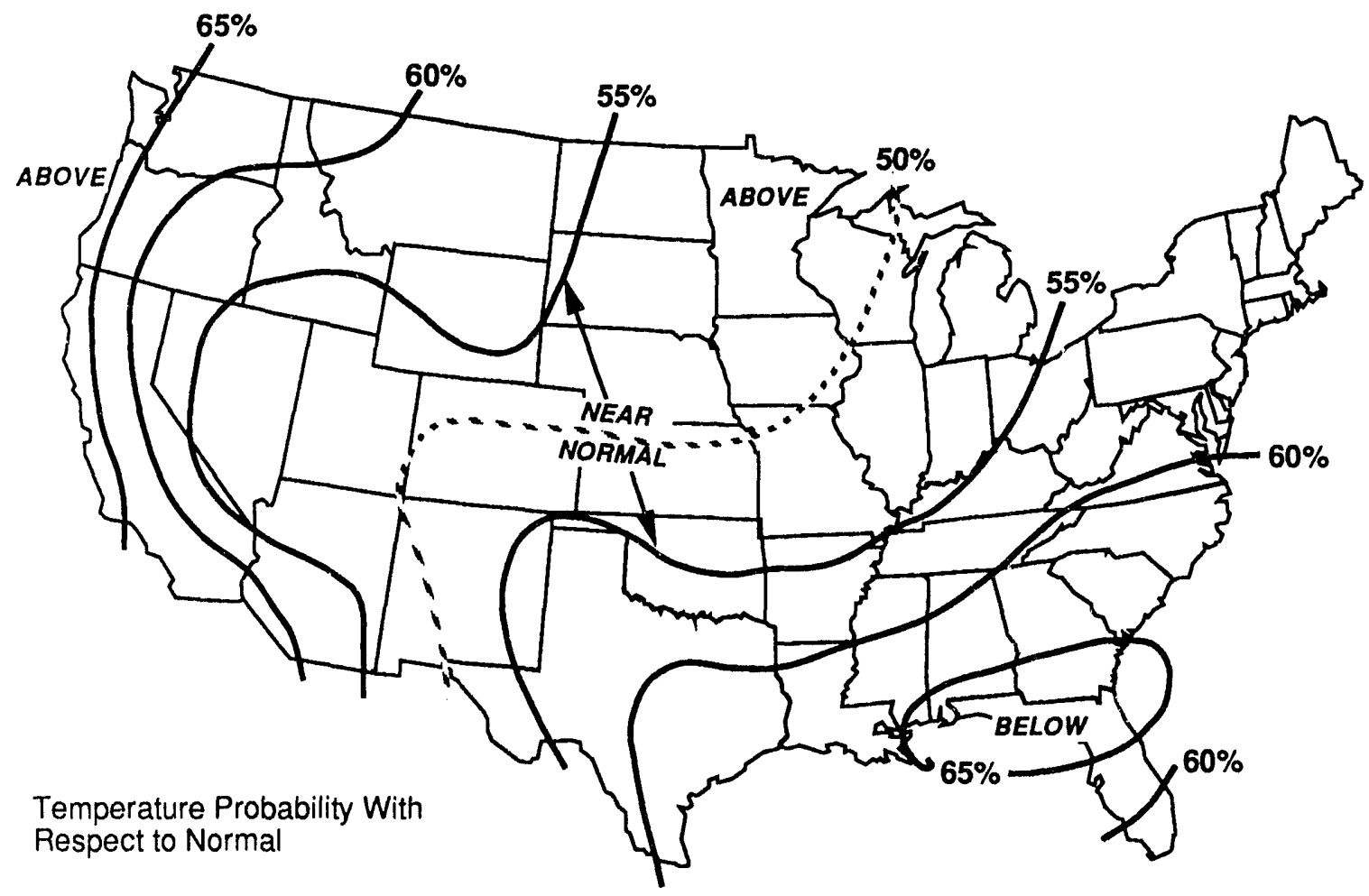

Source: National Weather Senvice, National Oceanic and Atmospheric Administration, U.S. Department of Commerce. 
Table 13. U.S. Total Heating Degree Days by City

(Population Weighted Heating Degree-Days, Except Where Noted)

\begin{tabular}{|c|c|c|c|c|c|}
\hline \multirow[b]{2}{*}{ Location } & \multirow{2}{*}{$\begin{array}{c}\text { Current } \\
07 / 01 / 93 \\
\text { thru } \\
02 / 19 / 94\end{array}$} & \multirow{2}{*}{$\begin{array}{c}\text { Prevlous } \\
07 / 01 / 92 \\
\text { thru } \\
02 / 19 / 93\end{array}$} & \multirow{2}{*}{$\begin{array}{c}\text { Normal } \\
07 / 01 \\
\text { thru } \\
02 / 19\end{array}$} & \multicolumn{2}{|c|}{ Percent Change } \\
\hline & & & & $\begin{array}{c}\text { Current } \\
\text { vs. } \\
\text { Prevlous }\end{array}$ & $\begin{array}{c}\text { Current } \\
\text { vs. } \\
\text { Normal }\end{array}$ \\
\hline U.S. Total, Population-Weighted & 3,165 & 2,892 & 3,021 & 9 & 5 \\
\hline Albuquerque & 2,922 & 2,929 & 3,097 & 0 & .6 \\
\hline Amarillo & 3,163 & 3,175 & 2,950 & 0 & 7 \\
\hline Asheville & 3,109 & 2,687 & 2,925 & 16 & 6 \\
\hline Atlanta & 2,060 & 1,885 & 2,160 & 9 & .5 \\
\hline Billings & 4,574 & 4,828 & 4,679 & -5 & -2 \\
\hline Boise & 3,919 & 4,147 & 3,926 & -5 & $\overline{0}$ \\
\hline Boston & 3,865 & 3,604 & 3,525 & 7 & 10 \\
\hline Buffalo & 4,640 & 4,123 & 4,217 & 13 & 10 \\
\hline Cheyenne & 4,764 & 4,619 & 4,564 & 3 & 4 \\
\hline Chicago & 4,665 & 4,115 & 4,303 & 13 & 8 \\
\hline Cincinnati & 3,872 & 3,289 & 3,546 & 18 & 9 \\
\hline Cleveland & 4,305 & 3,663 & 3,947 & 18 & 9 \\
\hline Columbia,SC & 2,007 & 1,807 & 1,933 & 11 & 4 \\
\hline Denver & 3,870 & 4,121 & 3,895 & -6 & -1 \\
\hline Des Moines & 4,853 & 4,335 & 4,422 & 12 & 10 \\
\hline Detroit & 4,399 & 3,921 & 4,238 & 12 & 4 \\
\hline Fargo & 6,595 & 6,137 & 6,168 & 7 & 7 \\
\hline Hartford & 4,387 & 4,013 & 4,018 & 9 & 9 \\
\hline Houston & 1,233 & 992 & 1,207 & 24 & 2 \\
\hline Jacksonville, FL & 1,104 & 789 & 1,074 & 40 & 3 \\
\hline Kansas City & 3,897 & 3,614 & 3,726 & 8 & 5 \\
\hline Las Vegas & 1,680 & 1,780 & 1,754 & -6 & .4 \\
\hline Los Angeles & 540 & 679 & 797 & -20 & -32 \\
\hline Memphis & 2,339 & 2,050 & 2,239 & 14 & 4 \\
\hline Miami & 78 & 20 & 162 & 290 & -52 \\
\hline Milwaukee & 4,603 & 4,250 & 4,656 & 8 & -1 \\
\hline Minneapolis & 5,832 & 5,174 & 5,365 & 13 & 9 \\
\hline Montgomery & 1,827 & 1,452 & 1,654 & 26 & 10 \\
\hline New York & 3,321 & 3,005 & 3,133 & 11 & 6 \\
\hline Oklahoma City & 2,903 & 2,570 & 2,628 & 13 & 10 \\
\hline Omaha & 4,797 & 4,344 & 4,390 & 10 & 9 \\
\hline Philadelphia & 3,152 & 2,876 & 3,257 & 10 & -3 \\
\hline Phoenix & 747 & 725 & 966 & 3 & -23 \\
\hline Pittsburgh & 4,088 & 3,525 & 3,900 & 16 & 5 \\
\hline Portland, ME & 4,855 & 4,628 & 4,627 & 5 & 5 \\
\hline Providence & 3,949 & 3,621 & 3,675 & 9 & 7 \\
\hline Raleigh & 2,536 & 2,278 & 2,412 & 11 & 5 \\
\hline Richmond & 2,799 & 2,519 & 2,736 & 11 & 2 \\
\hline St. Louis & 3,437 & 3,073 & 3,294 & 12 & 4 \\
\hline Salem, OR & 2,832 & 2,878 & 3,045 & -2 & -7 \\
\hline Salt Lake City & 3,677 & 4,089 & 3,847 & -10 & -4 \\
\hline San Francisco & 1,472 & 1,465 & 1,831 & 0 & -20 \\
\hline Seattle & 2,909 & 2,912 & 3,049 & 0 & -5 \\
\hline Shreveport & 1,794 & 1,637 & 1,696 & 10 & 6 \\
\hline Washington, DC & 3,095 & 2,724 & 2,754 & 14 & 12 \\
\hline
\end{tabular}

$* *=$ Normal heating degree-days 100 or less, or ratio incalculable.

Note: - The weather for the Nation, as measured by population-weighted heating degree-days from July 1, 1993, through February 19, 1994, has been 9 percent cooler than last year and 5 percent cooler than normal. - The total heating degree-days for the previous heating season (July 1,1992 . June 30,1993 ) was 4663 , and the normal is 4689 . - A new method for calculating heating/coolirg degree days was implemented by the Climate Analysis Center in October 1993, with further refinements implemented in November 1993. The routines incorporate 1961.1990 normals supplied by the National Climatic Data Center, and 1990 census data for calculation of population weighted degree days.

- Heating degree-days is defined as the number of degrees per day the daily average temperature is below 65 degrees Fahrenheit. The daily average temperature is the mean of the maximum and minimum temperature for a 24 -hour period.

Source: Weather data reported in the Winter Fuels Report are taken directly from a computerized system implemented by the National Oceanic and Atmospheric Administration, Department of Commerce. The National Oceanic and Atmospheric Administration (NOAA)/NWS, as a U.S. Government Agency, does not endorse any consumer information services. 


\title{
Appendix A
}

\author{
District \\ Descriptions \\ and Maps
}

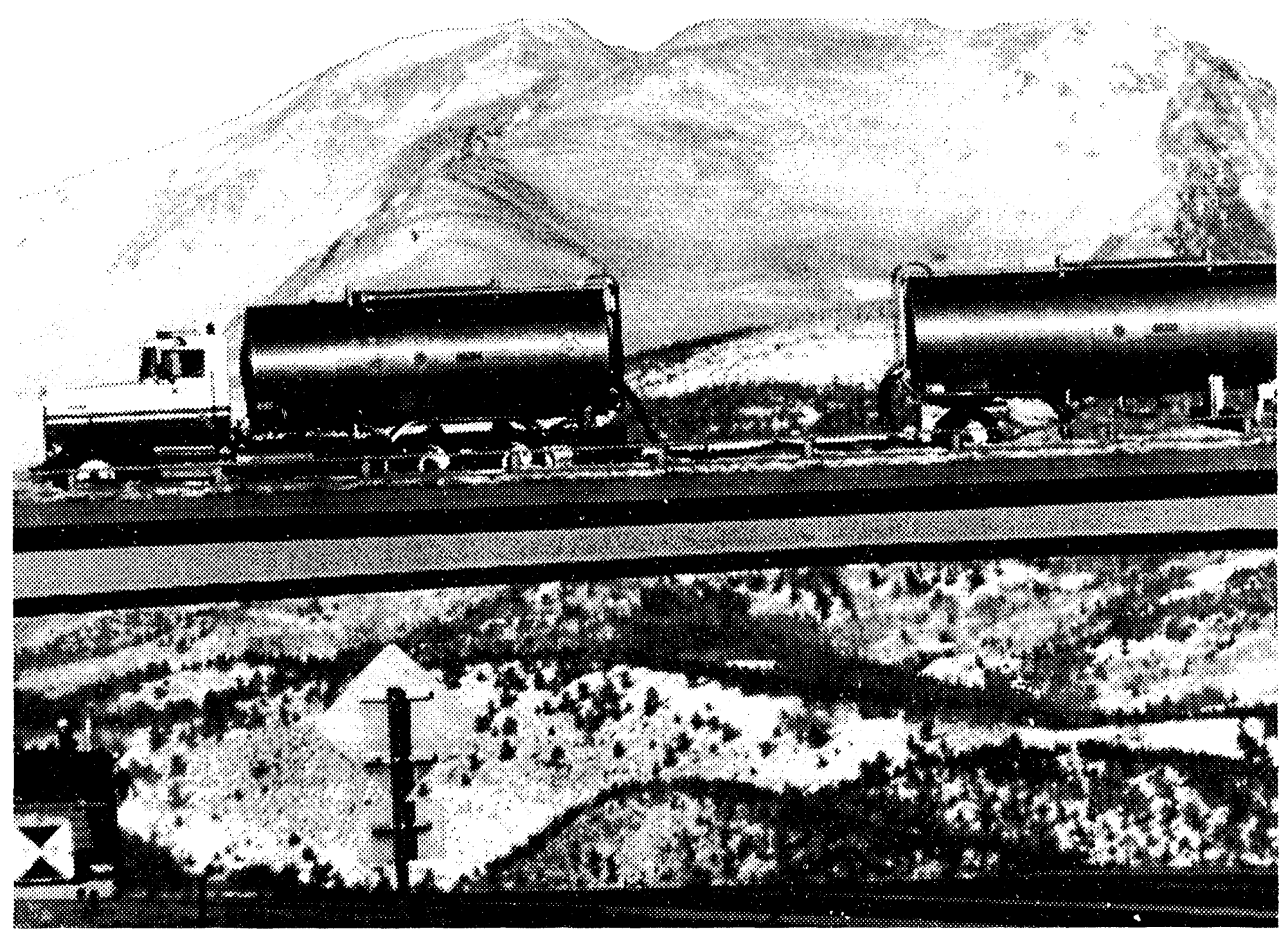

Tank trucks are used to distribute heating oil to remote areas. 


\section{Appendix A}

\section{District Descriptions and Maps}

The following are the Petroleum Administration for Defense (PAD) Districts.

\section{PAD District I}

East Coast: District of Columbia and the States of Maine, New Hampshire, Vermont, Massachusetts, Rhode Island, Connecticut, New Jersey, Delaware, Maryland, Virginia, North Carolina, South Carolina, Georgia, Florida, and the following counties of the State of New York: Cayuga, Tompkins, Chemung, and all counties east and north thereof. Also the following counties in the State of Pennsylvania: Bradford, Sullivan, Columbia, Montour, Northu:nberland, Dauphin, York, and all counties east thereof.

Appalachian No. 1: The State of West Virginia and those parts of the States of Pennsylvania and New York not included in the East Coast District.

\section{Sub-PAD District I}

New England (PADD 1X): The States of Connecticut, Maine, Massachusetts, New Hampshire, Rhode Island, and Vermont.

Central Atlantic (PADD IY): The District of Columbia and the States of Delaware, Maryland, New Jersey, New York, and Pennsylvania.

Lower Atlantic (PADD 1Z): The States of Florida, Georgia, North Carolina, South Carolina, Virginia, and West Virginia.

\section{PAD District II}

Indiana-Illinois-Kentucky: The States of Indiana, Illinois, Kentucky, Tennessee, Michigan, and Ohio.

Minnesota-Wisconsin-North and South Dakota: The States of Minnesota, Wisconsin, North Dakota, and South Dakota.

Oklahoma-Kansas-Missouri: The States of Oklahoma, Kansas, Missouri, Nebraska, and Iowa.

\section{PAD District III}

Texas Inland: The State of Texas except the Texas Gulf Coast District.

Texas Gulf Coast: The following counties of the State of Texas: Newton, Orange, Jefferson, Jasper, Tyler, Hardin, Liberty, Chambers, Polk, San Jacinto, Montgomery, Harris, Galveston, Waller, Fort Bend, Brazoria, Wharton, Matagorda, Jackson, Victoria, Calhoun, Refugio, Aransas, San Patricio, Nueces. Kleberg, Kenedy, Willacy, and Cameron.

Louisiana Gulf Coast: The following parishes of the State of Louisiana: Vernon, Rapides, Avoyelles, Pointe Coupee, West Feliciana, East Feliciana, Saint Helena, Tangipahoa, Washington, and all parishes south thereof. Also the following counties of the State of Mississippi: Pearl River, Stone, George, Hancock, Harrison, and Jackson. Also the following counties of the State of Alabama: Mobile and Baldwin.

North Louisiana-Arkansas: The State of Arkansas and those parts of the States of Louisiana, Mississippi, and Alabama not included in the Louisiana Gulf Coast District.

New Mexico: The State of New Mexico.

\section{PAD District IV}

Rocky Mountain: The States of Montana. Idaho, Wyoming. Utah, and Colorado.

\section{PAD District V}

West Coast: The States of Washington, Oregon. California, Nevada, Arizona, Alaska, and Hawaii. 


\section{Petroleum Administration for Defense (PAD) Districts}

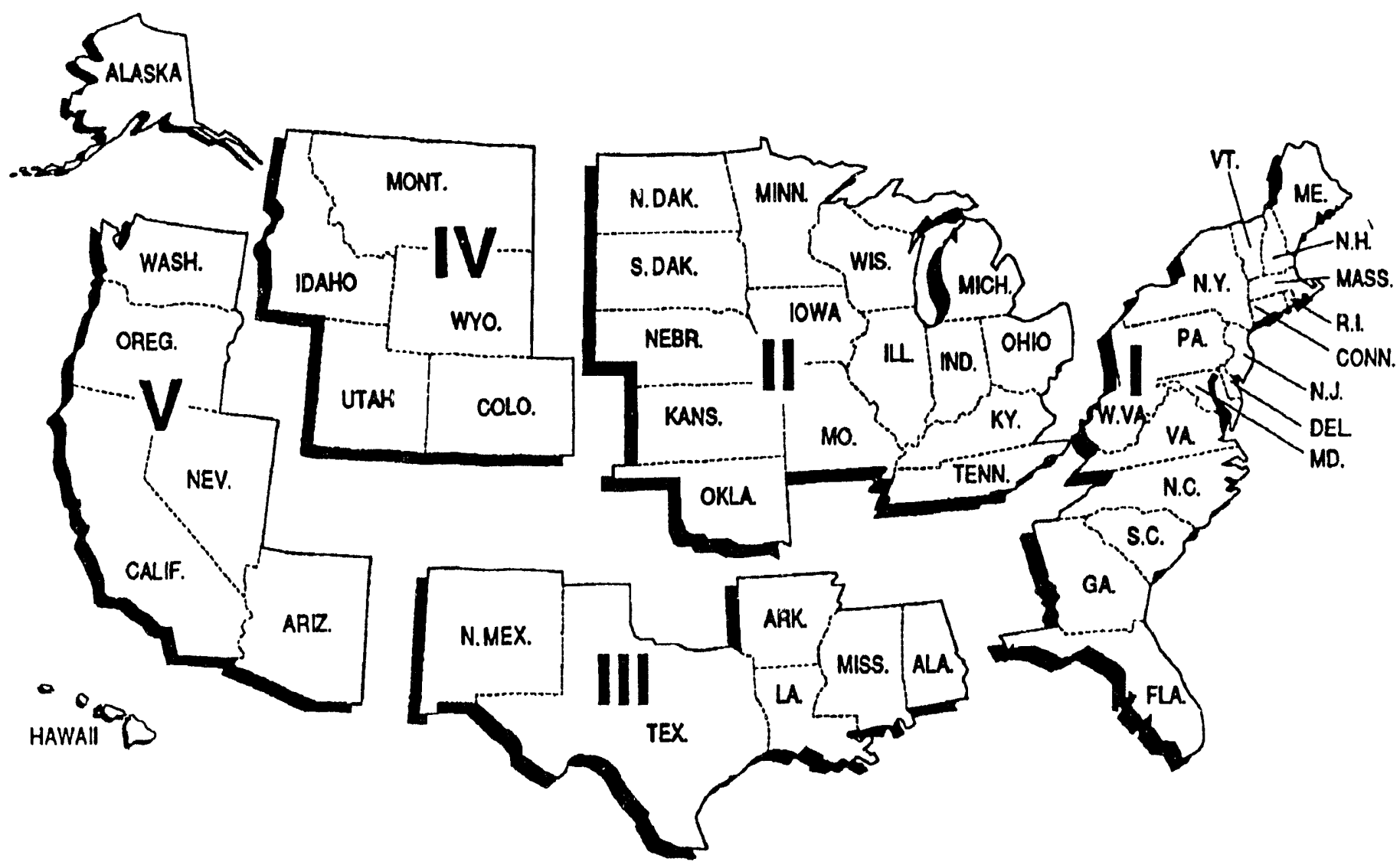




\section{Appendix B}

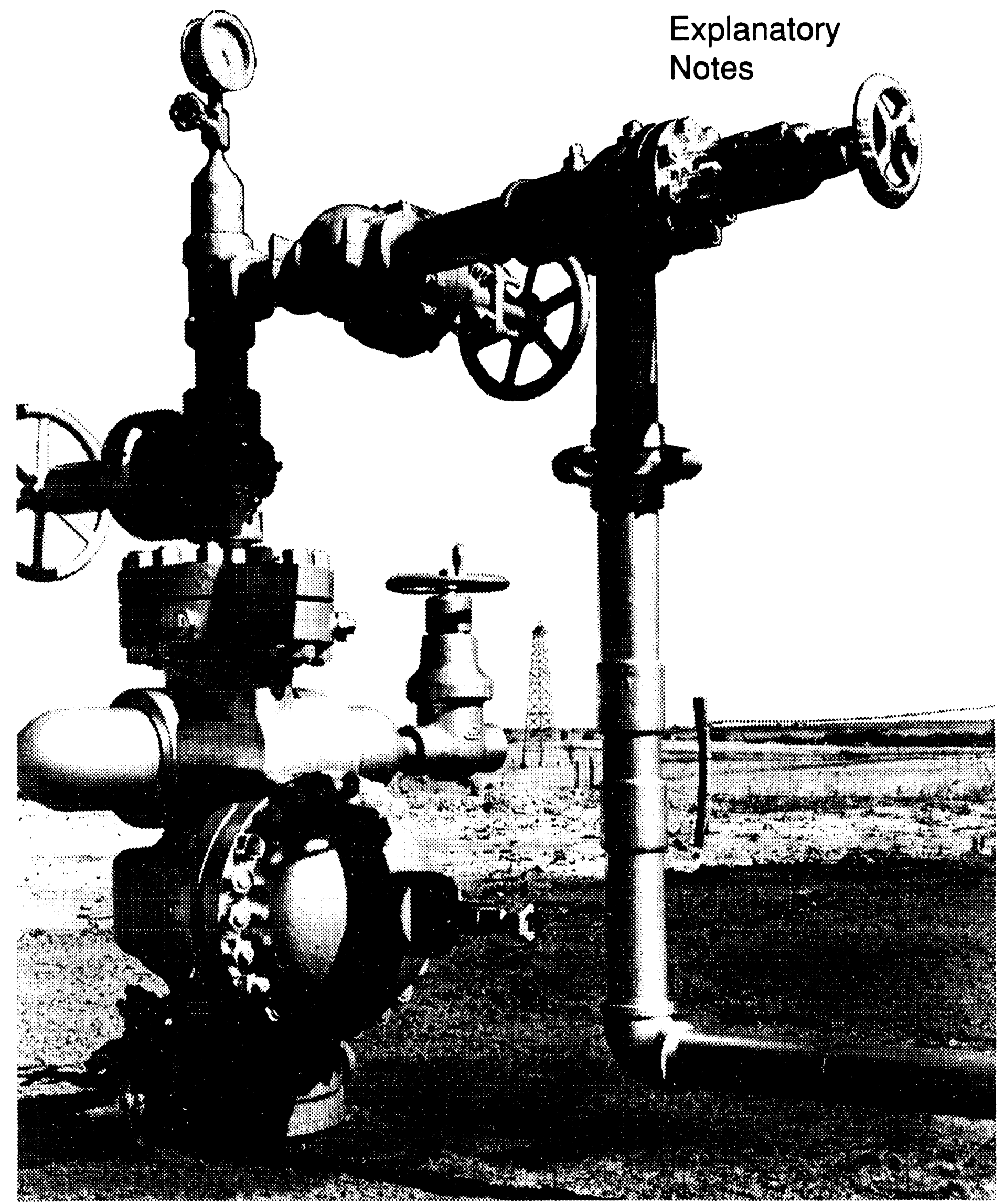

The cluster of pipes and valves that control the flow of oil at the mouth of an oil well is what oilmen call a

"Christmas Tree." 


\section{Explanatory Notes}

The following Explanatory Notes are provided to assist in understanding and interpreting the data presented in this publication.

- Note 1. Distillate Fuel Oil

- Note 2. Propane

- Note 3. Figures

- Note 4. Natural Gas

- Note 5. Prices

- Note 6. Provisions Regarding Confidentiality of Information

\section{Note 1. Distillate Fuel Oil}

Data on distillate fuel oil are collected within two time frames: weekly and monthly. Data from the Weekly Petroleum Supply Reporting System (WPSRS) are used to develop estimates for distillate fuel oil on a weekly basis. The forms that comprise the WPSRS are:

\section{Form}

Number

Name

EIA-800

\section{Weekly Refinery Report}

ElA-801 Weekly Bulk Terminal Report

EIA-802 Weekly Product Pipeline Report

EIA-803 Weekly Crude Oil Report

EIA-804 Weekly Imports Report

Monthly data are extracted from selected surveys in the Monthly Petroleum Supply Reporting System (MPSRS). The forms that comprise the MPSRS are:

\section{Form}

Number

Nane

EIA-810

\section{Monthly Refinery Report}

EIA-811 Monthly Bulk Terminal Report

EIA-812 Monthly Product Pipeline Report

EIA-814 Monthly lmports Report

EIA-816 Monthly Natural Gas Liquids Report

Refer to Explanatory Note 2 in the Petroleum Supply Monthly for a detailed discussion of the MPSRS.

\section{Sample Frame}

A sample of all petroleum companies report weekly data to the Energy Information Administration (EIA) on crude oil and petroleum product stocks, refinery inputs and production, and crude oil and petroleum product imports. The sample of companies that report weekly is selected from the universe of companies that report on the comparable monthly surveys.

\section{Sampling}

The sampling procedure used for the weekly system is the cut-off method. In the cut-off method, companies are ranked from largest to smallest on the basis of the quantities reported during some previous period. Companies are chosen for the sample beginning with the largest and adding companies until the total sample covers about 90 percent of the total, for each item and each geographic region for which weekly data are published.

\section{Collection Methods}

Data are collected by mail, mailgram, telephone, Telex, Telefax, and electronic transmission on a weekly basis. All canvassed firms must file by 5:00 p.m. on the Monday following the close of the report week, 7:00 a.m. Friday.

\section{Resubmissions}

During the processing week, company corrections of the prior week's data are also entered. This revised data is used to edit the current processing week's data.

\section{Estimation and Imputation}

After the company reports have been checked and entered into the weekly data base, explicit imputation is done for companies which have not yet responded. The imputed values atre exponentially smoothed means of recent weekly reported values for this specific company. The imputed values are treated like reported values in the estimation procedure, which calculates ratio estimates of the weekly totals. First, the current week's data for a given product reported by companies in a geographic region are summed. (Call this weekly sum, $W_{s}$ ) Next, the most recent month's data for the product reported by those sime companies are summed. (Call this monthly sum, $\mathrm{M}_{\mathrm{s}}$ ) Finally, let $M_{1}$ be the sum of most recent month's data for the product as reported by all companies. Then, the current week's ratio estimate for that product for all companies, $W_{1}$, is given by:

$$
W_{t}=\frac{M_{t}}{M_{s}} \cdot W_{s}
$$

This procedure is used directly to estimate total weekly inputs to refineries and production. To estimate stocks of finished products, the preceding procedure is followed separately for refineries, bulk terminals, and pipelines. Total estimates are formed by summing over establishment types. 
Weekly imports data are highly variable on a company-by-company basis or a week-by-week basis. Therefore, an exponentially smoothed ratio has been developed. The estimate of total weekly imports is the product of the smoothed ratio and the sum of the weekly reported values and imputed values.

\section{Response Rates}

The response rate as of the day after the filing deadline is about 80 percent for the EIA-800, 75 percent for the EIA-801, 95 percent for the EIA-802, 80 percent for the EIA-803, and greater than 95 percent for the EIA-804. However, more forms are received the next day, bringing the final response rates up. Late respondents are contacted by telephone. Nearly all of the major companies report on time. The nonresponse rate for the published estimate is usually between 1 percent and 2 percent.

\section{Note 2. Propane}

The Form EIA-807, "Propane Telephone Survey," was implemented in April 1990 as the result of the 1989 propane supply disruption. The hardships experienced by propane users during the December 1989 cold-snap in the Northeast and Mid-Continent areas made the need for timely supply information imperative. During 1990, propane data was collected and provided to Congress and others upon request. Because of the overwhelming demand for continuous monitoring of propane supply, the Winter Fuels Report was implemented in September 1990. This report publishes weekly data on propane as well as other heating fuels.

\section{Respondent Frame}

The Form EIA-807, "Propane Telephone Survey," collects data on production, stocks, and imports of propane. The sample of companies that report weekly is selected from the universe of companies that report on the comparable monthly surveys. These surveys are:

Form

Number Name

EIA-810 Monthly Refinery Report

EIA-811 Monthly Bulk Terminal Report

EIA-812 Monthly Product Pipeline Report

EIA-814 Monthly Imports Report

EIA-816 Monthly Natural Gas Liquids Report

\section{Sampling}

The sampling procedure used for the EIA-807 is the cut-off method. In the cut-off method, facilities are ranked from largest to sminilest on the basis of quantities reported for propane production, imports, and stocks. Companies are chosen for the sample beginning with the largest and adding companies until the total sample covers about 90 percent of the total for each item and each geographic region (Petroleum Administration for
Defense Districts I (IX, IY, IZ), II and III) for which data are published. A bench mark factor is used to capture the remaining 10 percent of the propane industry.

The sample frame for the EIA-807 is re-evaluated on an annual basis to assure 90 percent coverage of the total for each item collected and each geographic region. However, when necessary the sample frame is updated more frequently.

\section{Collection Methods}

Data are collected by telephone or facsimile. No written confirmation of the data submission is necessary. For weckly data collections, telephone calls to the respondents start on the Monday following the end of the report period. For monthly data collections, telephone calls to respondents start on the third working day following the end of the report period.

\section{Resubmissions}

Resubmissions are any changes to originally submitted data. A determination is made on whether to process the resubmissions based on the magnitude of the revision. Cell entries on publication tables are marked with an " $R$ " for revised.

\section{Revision Error}

Summary information on the revision error between preliminary weekly data and final monthly data will be incorporated in the feature article in the Petroleum Supply Monthly entitled, "Timeliness and Accuracy of Petroleum Supply Data." The last article was published in the August 1993 issue and evaluated the accuracy of the data for 1992 compared with previous years.

From October 1992 through March 1993, the difference between preliminary and final data for propane stocks remained within six percent. No difference in preliminary and final data was found for imports and production of propane.

\section{Estimation and Imputation}

After the company reports have been checked and entered into the EIA-807 data base, imputation is done for companies which have not yet .esponded. The imputed values are equal to the latest reported data for a particular reporting unit. Response rates are over 90 percent so very little imputation is done.

After the data files have been edited and corrected, aggregation is done for net production, imports, and stocks by each geographic region. Estimation factors, which were derived from 1992 reported data, are then applied to each cell to generate published estimates.

\section{Response Rate}

The response rate is generally 95 to 100 percent. Chronic nonrespondents and late filing respondents are contacted by telephone and reminded of their requirement to report. Nearly 
all of the major companies report on time. The nonresponse rate for the published estimate is usually between 1 percent and 2 percent.

\section{Note 3. Figures}

The national inventory (stocks) graphs for distillate fuel oil and propane include features to assist in comparing current inventory levels with past inventory levels and with judgments of critical levels. Methods used in developing the average inventory levels and minimum operating levels are described below.

\section{Average Inventory Levels}

The charts displaying inventory levels of distillate fuel oil and propane (Figures 1 through 14) provide the reader with actual inventory data compared to an "average range" for the most recent 3-year period running from January through December or from July through June. The ranges also reflect seasonal variation for the past 7 years.

The seasonal factors, which determine the shape of the upper and lower curves, are estimated with a seasonal adjustment technique developed at the Bureau of Census (Census X-11). The seasonal factors are assumed to be stable (i.e., unchanging from year to year) and additive (i.e., the series is deseasonalized by subtracting the seasonal factor for the appropriate month from the reported inventory levels.) The intent of deseasonalization is to remove only annual variation from the data. Thus, deseasonalized series would contain the same trends, cyclical components, and irregularities as the original data. The seasonal factors are updated annually in October, using the 7 most recent years' final monthly data.

The seasonal factors are used to deseasonalize data from the most recent 3-year period (January-December or July-June). The average of the deseasonalized 36-month series determines the midpoint of the "average range." The standard deviation of the deseasonalized 36 months is then calculated after adjusting for extreme data points. The upper curve of the "average range" is defined as average plus the seasonal factors plus the standard deviation. The lower curve is defined as the average plus the seasonal factors minus the standard deviation. Thus, the width of the "average range" is twice the standard deviation. The ranges are updated every 6 months in April and October.

The lines labeled "observed minimum" on the stock graphs are the lowest inventory levels observed during the most recent 36-month period as published in the Petroleum Supply Monthly.

\section{Note 4. Natural Gas}

Data contained in tables in the Natural Gas Section are from tables published in the Natural Gas Monthly. Data are collected from the following surveys:

\section{Form EIA-191}

The Form EIA-191, "Underground Natural Gas Storage Report," collects storage data by State, field, and reservoir. There are approximately 400 operating reservoirs in the United States. owned by 97 companies. It is a multipart form that reports the quantities of gas in storage, injections and withdrawals, and the location (State and county) and capacity of underground storage reservoirs along with peak day sendout during the reporting period.

The response rate as of the filing deadline is approximately 20 percent. Data from the remaining 80 percent of respondents are received in writing and/or by telephone within 3 to 4 days after the filing deadline. All data supplied by telephone are subsequently filed in writing, generally within 15 days of the filing deadline. The final response rate is 100 percent.

\section{Form FERC-11}

The Form FERC-11, "Natural Gas Pipeline Company Monthly Statement," is a monthly regulatory reporting form. Form FERC-11 is filed by major interstate natural gas pipeline companies whose combined sales for resale and gas transported interstate or stored for a fee exceeded 50 billion cubic feet in the previous calendar year. Approximately 50 pipeline companies report data on Form FERC-11. Information is collected monthly by mail. Historically, the response rate has been 100 percent.

\section{Form FPC-14}

The Form FPC-14, "Annual Report for Importers and Exporters of Natural Gas," is filed annually by each organization or individual having authorization to import and export natural gas regardless of whether any imports or exports took place during the reporting year. In 1992, 375 companies met the reporting criteria, only 143 reported imports or exports of natural gas.

\section{Form EIA-857}

The Form EIA-857, "Monthly Report of Natural Gas Purchases and Deliveries to Consumers," is a mandatory report. Data collected on the Form EIA-857 include both price and volume data and are considered proprietary. A sample of 391 natural gas companies including interstate pipelines, intrastate pipelines, and local distribution companies report on the Form ElA-857. The sample is selected independently for each of the 50 States and the District of Columbia.

\section{Form EIA-176}

The Form EIA-176, "Annual Report of Natural and Supplemental Gas Supply and Disposition," survey universe includes approximately 1,800 companies and 2,096 responses. These companies are interstate and intrastate natural gas pipeline companies, investor and municipally owned natural gas distributors, synthetic natural gas plant operators, and field, well. 
or processing plant operators that deliver natural gas directly to consumers (including their own industrial facilities) and/or that transport gas to, across, or from a State border through field or gathering facilities.

\section{Note 5. Prices}

The residential No. 2 heating oil and propane prices (excluding taxes) for a given State are based on the results of telephone surveys of a sample of marketers and refiners. Data are collected under the Energy Information Administration (EIA) State Heating Oil and Propane Program.

\section{Sampling Methodology and Estimation Procedures}

To estimate aggregate propane and No. 2 heating oil price data for a State, the sample weight and volume sales data were applied to the reported price, summed and divided by the sum of the weighted volume:

$$
\sum_{j=1}^{s} \sum_{i=1}^{n_{j}} w_{i j} v_{i j} p_{i j} / \sum_{j=1}^{s} \sum_{i=1}^{n_{j}} w_{i j} v_{i j} . \quad \text { where } w=
$$

sample weight, $v=$ volume, $p=$ price, $i=$ respondent, $n_{j}=$ sample size of stratum $\mathrm{j}$, and $\mathrm{s}=$ number of strata, to obtain a volume weighted price.

The volume used for No. 2 heating oil is the company's residential sales volume for 1988 as reported on the EIA-863 "Petroleum Product Sales Identification Survey." The volume used for propane is the company's residential propane sales volume for the previous year obtained by Forn EIA-877, "Winter Heating Fuels Telephone Survey." during the first pricing period.

These fixed volume weights indicate the relative importance of the individual companies according to the size of their sales. Therefore, changes in the average price across time reflect only the change in the price being offered by the company, and not changes in the amounts sold. Price indexes constructed using fixed volumes, such as these annual sales, are known as Laspeyres Indexes. The alternative method of weighting, current weights, would require each company to report the number of gallons sold at the reported price each pricing period. This method is more burdensome on the companies and reflects prices over a period of time as compared to a point in time. Therefore, the calculation of average prices tends to lag behind the reference period. Indexes constructed from current period weights are known as Paasch Indexes.

Both methods of weighting are correct; they do, however, vary when current weights are changing. It has been argued that during periods of change, the Laspeyres method has a tendency to overestimate price changes, while the Paasche method tends to underestimate price changes.

In this survey, it is expected that the relative change in volumes monthly is small. Residential sales are not bulk in nature and do not tend to reflect discounts on price for large volume purchases. Absolute changes in volume within a year's time would more likely reflect demand and be consistent across companies within a geographical area.

\section{Rellabllity of the Data}

Two types of errors are associated with data produced from a sample survey---sampling errors and nonsampling errors. Sampling errers occur because the estimates are based on a sample rather than on a census. The particular sample used for the EIA-877 survey is one of a large number of samples of equal size which could have been selected from the sampling frame using the same sample design. Each of these samples would produce a different estimate. If the estimates were averaged over all possible samples, the result would be the same as the estimate derived from a census of the sampling frame. The sampling error is a measure of variability among the estimates from all possible samples and, thus, is a measure of the precision with which an estimate from a particular sample approximates the results of a census.

Nonsampling errors can be attributed to many sources: (1) inability to obtain complete information about all cases in the sample (i.e., nonresponse), (2) response errors, (3) definitional difficulties, (4) differences in the interpretation of questions, (5) mistakes in recording or coding the data obtained, and (6) other errors of collection, response, coverage, and estimation for missing data. These nonsampling errors also occur in complete censuses.

Although no direct measurement of the biases due to nonsampling errors can be obtained, precautionary steps were taken in all phases of the frame development and data collection. processing, and tabulation processes, in an effort to minimize their influence.

Data in Tables B1 and B2 are based on survey data which are subject to sampling errors. Coefficients of variation, which are estimates of sampling errors, are presented for the propane and No. 2 heating oil prices in the following tables for the $1993 / 94$ survey. The coefficients of variation $(\mathrm{CV})$ were estimated by:

$$
C V(\hat{P})=\frac{\sqrt{V A R(\hat{P})}}{\hat{P}}
$$

where:

$$
\begin{aligned}
& V A R(\hat{P})=\frac{1}{\sqrt{2}} \sum_{k} N_{k}\left(\frac{1-f_{k}}{n_{k}}\right) S_{k}^{2} \\
& S_{\hat{k}}^{2}=S_{k q}^{2}+\hat{P}^{2} S_{k v}^{2}-2 \hat{P} S_{k k v}^{2}
\end{aligned}
$$

for heating oil:

$$
\begin{aligned}
S_{k q}^{2} & =\frac{\sum_{i=1}^{n_{k}}\left(P_{i k} V_{i k}-\overline{P_{k} V_{k}}\right)^{2}}{n_{k}-1} \\
S_{k v}^{2} & =\frac{\sum_{i=1}^{n_{k}}\left(V_{i k}-\overline{V_{k}}\right)^{2}}{n_{k}-1}
\end{aligned}
$$




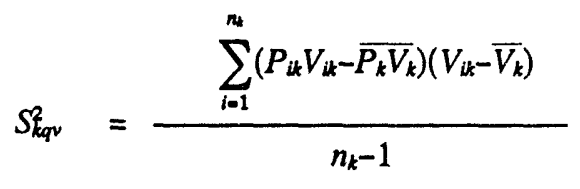

but for propane:

$$
S_{k q}^{2}=\frac{\sum_{i=1}^{n_{k}-1}\left(P_{i k} V_{i k}-P_{i+1, k} V_{i+1, k}\right)^{2}}{2\left(n_{k}-1\right)}
$$$$
S_{k}^{2}=\frac{\sum_{i=1}^{n_{k} \cdot 1}\left(V_{i k}-V_{i+1, k}\right)^{2}}{2\left(n_{k}-1\right)}
$$

$$
S_{k q v}^{2}=\frac{\sum_{i=1}^{n_{k}-1}\left(P_{i k} V_{i k}-P_{i+1, k} V_{i+1, k}\right)\left(V_{i k}-V_{i+1, k}\right)}{2\left(n_{k}-1\right)}
$$

$\mathrm{n}_{\mathrm{k}} \quad=$ number of respondents in stratum $\mathrm{k}$

$\mathrm{N}_{\mathrm{k}} \quad$ = number of population units in stratum $\mathrm{k}$

$V_{\mathrm{IK}} \quad=$ reported volume for unit $\mathrm{i}$ in stratum $\mathrm{k}$

$\bar{V}_{k} \quad=$ average volume for sample units in stratum $k$

$P_{i k} V_{i k}=$ reported revenue for unit $i$ in stratum $k$

$\bar{P}_{k} V_{k}=$ average revenue for sample units in stratum $k$

$\hat{\mathrm{P}} \quad=$ weighted average price for each State

\section{Residentlal No. 2 Heating OII}

For the No. 2 heating oil price data, a sample design similar to that used for the EIA Forrn EIA-782B, "Resellers'/Retailers' Monthly Petroleum Product Sales Report," sample design was used. The sampling frame was an extract of approximately

- 11,000 companies from the Form EIA-863, "Petroleum Product Sales Survey," conducted in 1989 and containing 1988 sales volume information. A one-way stratified sample design using No. 2 residential distillate frame sales volumes by State, for each of the 27 States to be sampled, was used. Stratum boundaries were determined by the Dalenius-Hodges procedure. Sample weights were calculated as the inverse of the probability $(\mathrm{N} / \mathrm{n})$. Certainty strata were established based on sales volumes and the number of States in which the company has sales. The expected price coefficient of variation is one to two percent.

\section{Residential Propane}

Since no volume sales information existed to predetermine the volume sales of propane dealers, two strata for propane dealers was used. A certainty stratum of the known, large, multi-State dealers was created. These companies were identified using establishment lists obtained in deriving the frame. All other dealers were in a second stratum and a random sample from this stratum was selected. Sample weights were calculated as the inverse of the probability $(\mathrm{N} / \mathrm{n})$. The name and address list sampling frame was constructed by first extracting from the Form EIA-863, "Petroleum Product Sales Identification Survey," companies who marked the box on the survey indicating they sell propane. This was augmented by companies on the Office of Oil and Gas Master File who have the words propane or liquefied petroleum gas (LPG) in their name. In addition, companies who file the Form EIA-782A, "Refiners'/Gas Plant Operators" Monthly Petroleum Product Sales Report," and report retail propane or the Form EIA-782C, "Monthly Report of Petroleum Products Sold into States for Consumption," and report propane, as well as companies that were active on the Form EIA-174. "Liquefied Petroleum Gas Survey," prior to its discontinuance. were included.

After unduplicating these companies, the initial frame file contained approximately 5,100 companies. Additional companies were obtained from an extract of a current Dun and Bradstreet file of SIC code 5984(9903), primary and secondary retail propane dealers, containing 3,283 names and addresses. Removal of duplicates within this file and between it and the initial frame file was performed using tailored automated match programs with manual review, and resulted in approximately 1,000 potential adds to the initial file. Similarly, additional names and addresses were furnished by industry associations and journais and by State Energy Offices, yielding another 7.429 names. Again, removal of duplicates through the match programs yielded an approximate potential add of 900 companies. Another 800 companies were identified as residing on the Master File but not previously selected as potential propane sellers. Further matching, merging and unduplicating reduced the final total frame count to approximately 6,000 companies. Reseller/retailer propane price data were unavailable to calculate a target coefficient of variation. However, it was expected that residential propane price variances were similar to heating oil. Increases in variances were expected as a result of lack of detailed stratification, but were only expected to reach three to four percent.

\section{Revision Error}

The numbers in Tables B3 and B4 display revision errors for heating oil and propane prices collected during the 1992/93 survey season. Numbers may be revised in the publication based on data received late or receipt of revised data. Numbers are published as preliminary and final. The difference between preliminary and final data is called the revision error.

\section{Response Rate}

Response rates are generally 95 to 100 percent. 
Table B1. Coefflcieints of Varlation for Residential Heating Oll Prices by Region and State (Cents per Gallon)

\begin{tabular}{|c|c|c|c|c|c|c|c|c|c|c|c|c|}
\hline \multirow[b]{2}{*}{ Reglon/State } & \multicolumn{12}{|c|}{ 1993/94 Heating Season } \\
\hline & $10 / 04$ & $10 / 18$ & $11 / 01$ & $11 / 15$ & $12 / 06$ & $12 / 20$ & $01 / 03$ & $01 / 17$ & $01 / 31$ & $02 / 07$ & $02 / 14$ & $02 / 21$ \\
\hline Average & 0.01 & 0.01 & 0.01 & 0.01 & 001 & 001 & $00 \%$ & $00 \%$ & 0.01 & 001 & & \\
\hline Eastcoast (OADDI) $/$, & 0002 & 0.01 & 0.01 & 0.01 & 0.01 & 0.01 & 0001 & $0.01 \%$ & 0001 & 001 & & \\
\hline Now England (PADO $\mathrm{X})$ & 0.01 & 0.01 & 0.01 & 0.01 & 0.01 & 0.01 & 0.01 & 0.01 & 001 & 8001 & & \\
\hline Connecticut & 0.01 & 0.01 & 0.01 & 0.01 & 0.00 & 0.01 & 0.01 & 0.01 & 0.01 & 0.01 & & \\
\hline Maine & 0.01 & 0.00 & 0.01 & 0.01 & 0.01 & 0.01 & 0.01 & 0.01 & 0.01 & 0.00 & & \\
\hline Massachusetts & 0.02 & 0.01 & 0.01 & 0.01 & 0.01 & 0.01 & 0.01 & 0.01 & 0.01 & 0.01 & & \\
\hline New Hampshire & 0.01 & 0.01 & 0.01 & 0.01 & 0.01 & 0.01 & 0.01 & 0.01 & 0.01 & 0.01 & & \\
\hline Rhode Island & 0.01 & 0.01 & 0.01 & 0.01 & 0.00 & 0.01 & 0.01 & 0.00 & 0.00 & 0.00 & & \\
\hline Vermont & 0.02 & 0.02 & 0.02 & 0.02 & 0.02 & 0.02 & 0.02 & 0.02 & 0.01 & 0.01 & & \\
\hline Oentral Atantic (PADO $\mathrm{PY}$ & 0.02 & 0.02 & 0.02 & 0.02 & 0.02 & 0.02 & 002 & 002 & 002 & 002 & & \\
\hline Delaware & 0.00 & 0.01 & 0.01 & 0.01 & 0.01 & 0.01 & 0.01 & 0.01 & 0.01 & 0.01 & & \\
\hline District of Columbia & 0.00 & 0.00 & 0.00 & 0.00 & 0.00 & 0.00 & 0.00 & 0.00 & 0.00 & 0.00 & & \\
\hline Maryland & 0.01 & 0.01 & 0.01 & 0.01 & 0.01 & 0.01 & 0.01 & 0.01 & 0.01 & 0.01 & & \\
\hline New Jersey & 0.02 & 0.02 & 0.02 & 0.02 & 0.02 & 0.02 & 0.02 & 0.02 & 0.02 & 0.02 & & \\
\hline New York & 0.03 & 0.03 & 0.03 & 0.03 & 0.03 & 0.03 & 0.03 & 0.03 & 0.02 & 0.02 & & \\
\hline Pennsylvania & 0.02 & 0.02 & 0.02 & 0.02 & 0.02 & 0.02 & 0.02 & 0.02 & 0.01 & 0.01 & & \\
\hline Lower Allantic (PADD /Z) & 0.01 & 0.01 & 0,01 & 0.01 & 0.01 & 0.01 & 0.01 & 0.01 & 0.01 & 0.01 & & \\
\hline North Carolina & 0.01 & 0.01 & 0.01 & 0.01 & 0.01 & 0.01 & 0.01 & 0.01 & 0.01 & 0.01 & & \\
\hline Virginia & 0.01 & 0.01 & 0.01 & 0.01 & 0.01 & 0.01 & 0.01 & 0.01 & 0.01 & 0.01 & & \\
\hline Midwest (PADD II) & 0.00 & 0.01 & 0.01 & 0.01 & 0.01 & 0.01 & 0.01 & 001 & 0.01 & 0.01 & & \\
\hline Indiana & 0.02 & 0.01 & 0.02 & 0.02 & 0.02 & 0.02 & 0.02 & 0.02 & 0.02 & 0.02 & & \\
\hline lowa & 0.01 & 0.00 & 0.01 & 0.00 & 0.01 & 0.00 & 0.01 & 0.00 & 0.01 & 0.01 & & \\
\hline Michigan & 0.01 & 0.01 & 0.01 & 0.01 & 0.01 & 0.01 & 0.01 & 0.01 & 0.01 & 0.01 & & \\
\hline Minnesota & 0.02 & 0.02 & 0.02 & 0.02 & 0.02 & 0.03 & 0.03 & 0.03 & 0.03 & 0.03 & & \\
\hline Ohio & 0.01 & 0.01 & 0.01 & 0.01 & 0.01 & 0.01 & 0.01 & 0.01 & 0.01 & 0.01 & & \\
\hline Wisconsin & 0.01 & 0.01 & 0.01 & 0.01 & 0.01 & 0.01 & 0.01 & 0.01 & 0.01 & 0.01 & & \\
\hline
\end{tabular}

Source: Based on data collected by State Energy Offices. 
Table B2. Coefficients of Variation for Residentlal Propane Prices by Region and State (Cents per Gallon)

\begin{tabular}{|c|c|c|c|c|c|c|c|c|c|c|c|c|}
\hline \multirow[b]{2}{*}{ Reglon/State } & \multicolumn{12}{|c|}{ 1993/94 Heating Season } \\
\hline & $10 / 04$ & $10 / 18$ & $11 / 01$ & $11 / 15$ & $12 / 06$ & $12 / 20$ & $01 / 03$ & $01 / 17$ & $01 / 31$ & $02 / 07$ & $02 / 21$ & $03 / 07$ \\
\hline Average & 001 & 0.01 & 0.01 & 0.01 & 0.01 & 0.01 & 0.01 & 0.01 & 0.01 & 0.01 & & \\
\hline East Coast PABOII & 0.02 & 0.01 & 0.01 & 0.01 & 0.01 & 0.01 & 0.01 & 0.01 & 0.01 & 0.01 & & \\
\hline Now England $(\mathrm{PADON} X)$ & 0.03 & 0.01 & 0.02 & 0.01 & 0.01 & 001 & 0.01 & 0.01 & 0.01 & 0.01 & & \\
\hline Connecticut & 0.03 & 0.03 & 0.03 & 0.03 & 0.03 & 0.03 & 0.02 & 0.03 & 0.02 & 0.02 & & \\
\hline Maine & 0.05 & 0.05 & 0.05 & 0.05 & 0.05 & 0.05 & 0.05 & 0.05 & 0.05 & 0.05 & & \\
\hline Massachusetts & 0.05 & 0.03 & 0.03 & 0.03 & 0.03 & 0.03 & 0.03 & 0.03 & 0.03 & 0.03 & & \\
\hline New Hampshire & 0.02 & 0.03 & 0.03 & 0.03 & 0.03 & 0.03 & 0.03 & 0.03 & 0.03 & 0.04 & & \\
\hline Rhode Island & 0.01 & 0.00 & 0.00 & 0.01 & 0.00 & 0.00 & 0.00 & 0.00 & 0.00 & 0.00 & & \\
\hline Vermont & 0.03 & 0.03 & 0.03 & 0.03 & 0.03 & 0.03 & 0.03 & 0.03 & 0.03 & 0.03 & & \\
\hline Central atlantic (PADD IY) & 002 & $0.02 \%$ & 002 & 002 & 002 & 002 & 0.02 & 0.02 & 0,02 & 0.02 & & \\
\hline Delaware & 0.00 & 0.01 & 0.01 & 0.01 & 0.01 & 0.01 & 0.01 & 0.01 & 0.01 & 0.01 & & \\
\hline Maryland & 0.02 & 0.01 & 0.01 & 0.01 & 0.01 & 0.01 & 0.01 & 0.02 & 0.02 & 0.03 & & \\
\hline New Jersey & 0.03 & 0.01 & 0.01 & 0.01 & 0.01 & 0.01 & 0.01 & 0.01 & 0.02 & 0.01 & & \\
\hline New York & 0.03 & 0.03 & 0.03 & 0.03 & 0.03 & 0.03 & 0.03 & 0.03 & 0.03 & 0.03 & & \\
\hline Pennsylvania & 0.05 & 0.05 & 0.05 & 0.05 & 0.04 & 0.04 & 0.04 & 0.04 & 0.04 & 0.04 & & \\
\hline Lower Atlantic $(P A D D$ IZ) & 002 & 0.02 & 0.01 & 0.01 & 0.01 & 0.01 & 0.01 & 0.01 & 0.01 & 0.01 & & \\
\hline North Carolina & 0.03 & 0.02 & 0.02 & 0.02 & 0.02 & 0.02 & 0.02 & 0.01 & 0.01 & 0.01 & & \\
\hline Virginia & 0.04 & 0.03 & 0.03 & 0.02 & 0.02 & 0.02 & 0.02 & 0.02 & 0.02 & 0.02 & & \\
\hline Mldwest (PADD II) & 0.00 & 0.01 & 0.01 & 0.01 & 0.01 & 0.01 & 0.01 & 0.01 & 0.01 & 0.01 & & \\
\hline Indiana & 0.03 & 0.02 & 0.02 & 0.02 & 0.02 & 0.02 & 0.02 & 0.02 & 0.03 & 0.03 & & \\
\hline lowa & 0.03 & 0.02 & 0.02 & 0.02 & 0.02 & 0.02 & 0.02 & 0.02 & 0.02 & 0.02 & & \\
\hline Kansas & 0.03 & 0.03 & 0.03 & 0.03 & 0.03 & 0.03 & 0.03 & 0.03 & 0.03 & 0.03 & & \\
\hline Michigan & 0.02 & 0.02 & 0.02 & 0.02 & 0.02 & 0.02 & 0.02 & 0.02 & 0.02 & 0.02 & & \\
\hline Minnesota & 0.03 & 0.02 & 0.02 & 0.02 & 0.02 & 0.03 & 0.02 & 0.02 & 0.02 & 0.02 & & \\
\hline Missouri & 0.03 & 0.02 & 0.02 & 0.02 & 0.02 & 0.02 & 0.02 & 0.02 & 0.02 & 0.02 & & \\
\hline North Dakota & 0.02 & 0.02 & 0.02 & 0.02 & 0.03 & 0.03 & 0.02 & 0.02 & 0.02 & 0.02 & & \\
\hline Ohio & 0.05 & 0.04 & 0.04 & 0.03 & 0.03 & 0.03 & 0.03 & 0.03 & 0.03 & 0.03 & & \\
\hline South Dakota & 0.02 & 0.01 & 0.01 & 0.01 & 0.01 & 0.01 & 0.02 & 0.02 & 0.02 & 0.01 & & \\
\hline Wisconsin & 0.01 & 0.01 & 0.01 & 0.01 & 0.01 & 0.01 & 0.01 & 0.01 & 0.01 & 0.01 & & \\
\hline
\end{tabular}

Source: Based on data collected by State Energy Offices. 
Table B3. Revision Rates for Residential Heating Oil Prices by Reglon and State (Cents per Gallon)

\begin{tabular}{|c|c|c|c|c|c|c|c|c|c|c|c|c|}
\hline \multirow[b]{2}{*}{ Reglon/State } & \multicolumn{12}{|c|}{ 1992/93 Heating Season } \\
\hline & $10 / 05$ & $10 / 19$ & $11 / 02$ & $11 / 16$ & $12 / 07$ & $12 / 21$ & $01 / 04$ & $01 / 18$ & $02 / 01$ & $02 / 15$ & $03 / 01$ & $03 / 15$ \\
\hline Averago & 0.0 & 00 & 0,0 & 0.0 & 0.0 & 0.0 & 0.0 & 0.0 & 0.0 & 0.0 & 0.0 & 0.0 \\
\hline East Coast (PADD I) & 0.0 & 0.0 & 0.1 & 0.0 & 0.0 & 0.0 & 00 & 0.0 & 0.0 & 0.0 & 0.0 & 0.0 \\
\hline Now England (PADD IX) & 0.0 & 0.1 & 0.1 & 0.0 & 0.0 & 0.0 & 00 & 0.0 & 0.1 & 0.0 & 0.0 & 0.0 \\
\hline Connecticut & 0.0 & 0.0 & 0.0 & 0.0 & 0.0 & 0.0 & 0.0 & 0.0 & 0.0 & 0.0 & 0.0 & 0.0 \\
\hline Maine & 0.0 & 0.1 & 0.4 & 0.0 & 0.0 & 0.0 & 0.0 & 0.0 & 0.0 & 0.0 & 0.0 & 0.0 \\
\hline Massachusetts & 0.0 & 0.1 & 0.0 & 0.0 & 0.0 & 0.0 & 0.0 & 0.0 & 0.2 & 0.0 & 0.0 & 0.0 \\
\hline New Hampshire & 0.0 & 0.0 & 0.0 & 0.2 & 0.0 & 0.2 & 0.0 & 0.0 & 0.3 & 0.0 & 0.0 & 0.0 \\
\hline Rhode Island & 0.0 & 0.0 & 0.0 & 0.0 & 0.0 & 0.0 & 0.0 & 0.0 & 0.0 & 0.0 & 0.0 & 0.0 \\
\hline Verlilont & 0.0 & 0.0 & 0.0 & 0.0 & 0.0 & 0.0 & 0.0 & 0.0 & 0.0 & 0.0 & 0.0 & 0.0 \\
\hline Centra Allantio $(\mathrm{PADD} \cap Y)$ & 0.0 & 00 & 00 & 0.0 & 00 & 0.0 & 0.0 & 00 & 0.0 & 0.0 & 0.0 & 0.0 \\
\hline Delaware & 0.0 & 0.0 & 0.0 & 0.1 & 0.0 & 0.0 & 0.0 & 0.0 & 0.0 & 0.0 & 0.0 & 0.0 \\
\hline District of Columbia & 0.0 & 0.0 & 0.0 & 0.0 & 0.0 & 0.0 & 0.0 & 0.0 & 0.0 & 0.0 & 0.0 & 0.0 \\
\hline Maryland & 0.0 & 0.2 & 0.1 & 0.0 & 0.0 & 0.0 & 0.0 & 0.0 & 0.0 & 0.0 & 0.0 & 0.0 \\
\hline New Jersey & 0.0 & 0.0 & 0.0 & 0.0 & 0.1 & 0.0 & 0.0 & 0.0 & 0.0 & 0.0 & 0.0 & 0.0 \\
\hline Now York & 0.0 & 0.0 & 0.3 & 0.0 & 0.0 & 0.0 & 0.0 & 0.0 & 0.0 & 0.0 & 0.0 & 0.0 \\
\hline Pennsylvania & 0.0 & 0.5 & 0.0 & 0.0 & 0.0 & 0.0 & 0.0 & 0.0 & 0.0 & 0.0 & 0.1 & 0.0 \\
\hline Lower Atlantic (PADD IZ) & 0.0 & 12 & 00 & 0,0 & 00 & 0.1 & 0.0 & 00 & 0,0 & 0.0 & 0.0 & 0.0 \\
\hline North Carolina & 0.0 & 0.0 & 0.0 & 0.0 & 0.0 & 0.0 & 0.0 & 0.0 & 0.0 & 0.0 & 0.0 & 0.0 \\
\hline Virginia & 0.0 & 2.6 & 0.0 & 0.0 & 0.0 & 0.1 & 0.0 & 0.0 & 0.0 & 0.0 & 0.0 & 0.0 \\
\hline Midwest (PADD II) & 0.0 & 0.2 & 0.0 & 0.0 & 0.0 & 0.0 & 0.0 & 0,0 & 0.0 & 0.0 & 0.0 & 0.0 \\
\hline Indiana & 0.0 & 0.0 & 0.0 & 0.0 & 0.0 & 0.0 & 0.0 & 0.0 & 0.1 & 0.0 & 0.0 & 0.0 \\
\hline lowa & 0.0 & 0.0 & 0.0 & 0.0 & 0.0 & 0.0 & 0.0 & 0.0 & 0.0 & 0.0 & 0.0 & 0.0 \\
\hline Michigan & 0.0 & 0.4 & 0.0 & 0.0 & 0.0 & 0.0 & 0.0 & 0.0 & 0.0 & 0.0 & 0.0 & 0.0 \\
\hline Minnesota & 0.0 & 0.2 & 0.1 & 0.0 & 0.0 & 0.0 & 0.0 & 0.0 & 0.0 & 0.0 & 0.1 & 0.0 \\
\hline Ohio & 0.0 & 0.0 & 0.0 & 0.0 & 0.0 & 0.0 & 0.0 & 0.0 & 0.0 & 0.0 & 0.0 & 0.0 \\
\hline Wisconsin & 0.0 & 0.0 & 0.0 & 0.0 & 0.0 & 0.0 & 0.0 & 0.0 & 0.0 & 0.0 & 0.0 & 0.0 \\
\hline
\end{tabular}

Source: Based on data collected by State Energy Offices. 
Table B4. Revision Rates for Residential Propane Prices by Region and State (Cents per Gallon)

\begin{tabular}{|c|c|c|c|c|c|c|c|c|}
\hline \multirow[b]{2}{*}{ Reglon/State } & \multicolumn{8}{|c|}{ 1992/93 Heating Season } \\
\hline & $10 / 05$ & $10 / 19$ & $11 / 02$ & $11 / 16$ & $12 / 07$ & $12 / 21$ & $01 / 04$ & $01 / 11$ \\
\hline Averago $/$ la $/$ la $/$ । & 0.0 & 0.2 & $0.2 \%$ & 0.0 & 0. & $0,0 \%$ & 0.0 & 0.0 \\
\hline EastCoast (PAOOU) & 000 & 04 & 01 & $01 \%$ & 00 & 00 & 00 & 0.0 \\
\hline $\begin{array}{l}\text { Now England (PADD D } \\
\text { Connecticut } \\
\text { Maine } \\
\text { Massachusetts } \\
\text { New Hampshire } \\
\text { Rhode Island } \\
\text { Vermont }\end{array}$ & $\begin{array}{l}0.0 \\
0.0 \\
0.0 \\
0.0 \\
0.0 \\
0.0 \\
0.0\end{array}$ & $\begin{array}{l}0.0 \\
0.0 \\
0.1 \\
0.0 \\
0.0 \\
0.0 \\
0.0\end{array}$ & $\begin{array}{l}0.0 \\
0.0 \\
0.4 \\
0.0 \\
0.0 \\
0.0 \\
0.0\end{array}$ & $\begin{array}{l}0.4 \\
0.0 \\
0.1 \\
0.0 \\
0.1 \\
0.0 \\
1.9\end{array}$ & $\begin{array}{l}0.0 \\
0.0 \\
0.0 \\
0.1 \\
0.0 \\
0.6 \\
0.0\end{array}$ & $\begin{array}{l}0.0 \\
0.0 \\
0.0 \\
0.0 \\
0.0 \\
0.0 \\
0.0\end{array}$ & $\begin{array}{l}0.0 \\
0.0 \\
0.0 \\
0.0 \\
0.0 \\
0.0 \\
0.0\end{array}$ & $\begin{array}{l}0.0 \\
0.0 \\
0.0 \\
0.0 \\
0.0 \\
0.0 \\
0.0\end{array}$ \\
\hline $\begin{array}{l}\text { Contral Allantio (PADDM } \\
\text { Delaware } \\
\text { Maryland } \\
\text { New Jersey } \\
\text { New York } \\
\text { Pennsylvania }\end{array}$ & $\begin{array}{l}0.0 \\
0.0 \\
0.0 \\
0.0 \\
0.0 \\
0.0\end{array}$ & $\begin{array}{l}0.0 \\
0.0 \\
0.0 \\
1.5 \\
2.2 \\
4.8\end{array}$ & $\begin{array}{l}0.3 \\
0.0 \\
0.0 \\
0.0 \\
0.0 \\
0.6\end{array}$ & $\begin{array}{l}0.0 \\
0.0 \\
0.1 \\
0.0 \\
0.0 \\
0.0\end{array}$ & $\begin{array}{l}0.0 \\
0.0 \\
0.0 \\
0.0 \\
0.0 \\
0.0\end{array}$ & $\begin{array}{l}0.0 \\
0.0 \\
0.0 \\
0.0 \\
0.0 \\
0.0\end{array}$ & $\begin{array}{l}0.0 \\
0.0 \\
0.0 \\
0.0 \\
0.0 \\
0.0\end{array}$ & $\begin{array}{l}0.0 \\
0.0 \\
0.0 \\
0.0 \\
0.0 \\
0.0\end{array}$ \\
\hline $\begin{array}{l}\text { Lower Allantic }(\mathrm{PADO} / \mathrm{Z}) \\
\text { North Carolina } \\
\text { Virginia }\end{array}$ & $\begin{array}{l}0.0 \\
0.0 \\
0.0\end{array}$ & $\begin{array}{l}0.0 \\
0.0 \\
0.2\end{array}$ & $\begin{array}{l}0.0 \\
0.0 \\
0.0\end{array}$ & $\begin{array}{l}0.0 \\
0.0 \\
0.0\end{array}$ & $\begin{array}{l}0.0 \\
0.0 \\
0.0\end{array}$ & $\begin{array}{l}0.1 \\
0.1 \\
0.1\end{array}$ & $\begin{array}{l}0.0 \\
0.0 \\
0.0\end{array}$ & $\begin{array}{l}0.0 \\
0.0 \\
0.0\end{array}$ \\
\hline $\begin{array}{l}\text { Midwest (PADOI) } / \text { I } \\
\text { Indiana } \\
\text { lowa } \\
\text { Kansas } \\
\text { Michigan } \\
\text { Minnesota } \\
\text { Missouri } \\
\text { North Dakota } \\
\text { Ohio } \\
\text { South Dakota } \\
\text { Visconsin }\end{array}$ & $\begin{array}{l}0.0 \\
0.0 \\
0.0 \\
0.0 \\
0.0 \\
0.0 \\
0.0 \\
0.0 \\
0.0 \\
0.0 \\
0.0\end{array}$ & $\begin{array}{l}0.1 \\
0.0 \\
0.0 \\
1.0 \\
0.0 \\
0.1 \\
0.0 \\
0.0 \\
3.3 \\
0.0 \\
0.0\end{array}$ & $\begin{array}{l}0.2 \\
0.1 \\
0.0 \\
1.1 \\
0.1 \\
0.0 \\
0.6 \\
0.0 \\
0.0 \\
0.6 \\
0.0\end{array}$ & $\begin{array}{l}0.0 \\
0.0 \\
0.0 \\
0.0 \\
0.0 \\
0.0 \\
0.0 \\
0.0 \\
0.0 \\
0.0 \\
0.0\end{array}$ & $\begin{array}{l}0.0 \\
0.0 \\
0.0 \\
0.0 \\
0.0 \\
0.0 \\
0.0 \\
0.0 \\
0.0 \\
0.0 \\
0.3\end{array}$ & $\begin{array}{l}0.0 \\
0.0 \\
0.0 \\
0.0 \\
0.0 \\
0.0 \\
0.0 \\
0.0 \\
0.0 \\
0.0 \\
0.0\end{array}$ & $\begin{array}{l}0.0 \\
0.0 \\
0.0 \\
0.0 \\
0.0 \\
0.0 \\
0.0 \\
0.0 \\
0.0 \\
0.0 \\
0.0\end{array}$ & $\begin{array}{l}0.0 \\
0.0 \\
0.0 \\
0.0 \\
0.0 \\
0.0 \\
0.0 \\
0.0 \\
0.0 \\
0.0 \\
0.0\end{array}$ \\
\hline
\end{tabular}

\begin{tabular}{|c|c|c|c|c|c|c|c|c|}
\hline \multirow[b]{2}{*}{ Reglon/State } & \multicolumn{8}{|c|}{ 1992/93 Heating Season } \\
\hline & $01 / 18$ & $01 / 25$ & $02 / 01$ & $02 / 15$ & $03 / 01$ & $03 / 15$ & $04 / 05$ & $04 / 19$ \\
\hline Averago & $0.1 \%$ & 0.0 & 0.0 & 0.1 & 0.2 & 0.0 & 01 & 0.0 \\
\hline East Coast (PADO) & 0.0 & 0.0 & 0.0 & 0.0 & 0.6 & 0.0 & 0.0 & 0.0 \\
\hline $\begin{array}{l}\text { Now England (PADD }(X) \\
\text { Connecticut } \\
\text { Maine } \\
\text { Massachusetts } \\
\text { New Hampshire } \\
\text { Rhode Island } \\
\text { Vermont }\end{array}$ & $\begin{array}{l}0.0 \\
0.0 \\
0.0 \\
0.0 \\
0.0 \\
0.0 \\
0.0\end{array}$ & $\begin{array}{l}0.1 \\
0.0 \\
0.0 \\
0.0 \\
0.0 \\
0.0 \\
0.0\end{array}$ & $\begin{array}{l}0.0 \\
0.0 \\
0.0 \\
0.0 \\
0.0 \\
0.0 \\
0.0\end{array}$ & $\begin{array}{l}0.1 \\
0.1 \\
0.8 \\
0.0 \\
0.0 \\
0.0 \\
0.0\end{array}$ & $\begin{array}{l}0.0 \\
0.0 \\
0.0 \\
0.0 \\
0.1 \\
0.0 \\
0.0\end{array}$ & $\begin{array}{l}0.0 \\
0.0 \\
0.0 \\
0.0 \\
0.0 \\
0.0 \\
0.0\end{array}$ & $\begin{array}{l}0.0 \\
0.0 \\
0.0 \\
0.0 \\
0.0 \\
1.3 \\
0.0\end{array}$ & $\begin{array}{l}0.0 \\
0.0 \\
0.0 \\
0.0 \\
0.0 \\
0.0 \\
0.0\end{array}$ \\
\hline $\begin{array}{l}\text { Central Atlantic (PADDIY) } \\
\text { Delaware } \\
\text { Maryland } \\
\text { New Jersey } \\
\text { New York } \\
\text { Pennsylvanla }\end{array}$ & $\begin{array}{l}0.1 \\
0.0 \\
0.0 \\
0.0 \\
0.0 \\
0.2\end{array}$ & $\begin{array}{l}0.0 \\
0.0 \\
0.0 \\
0.0 \\
0.0 \\
0.0\end{array}$ & $\begin{array}{l}0.0 \\
0.0 \\
0.0 \\
0.0 \\
0.0 \\
0.1\end{array}$ & $\begin{array}{l}0.0 \\
0.0 \\
0.0 \\
0.0 \\
0.0 \\
0.3\end{array}$ & $\begin{array}{l}1.4 \\
0.0 \\
0.0 \\
0.0 \\
3.9 \\
0.0\end{array}$ & $\begin{array}{l}0.0 \\
0.0 \\
0.0 \\
0.0 \\
0.0 \\
0.0\end{array}$ & $\begin{array}{l}0.0 \\
0.0 \\
0.0 \\
0.2 \\
0.0 \\
0.0\end{array}$ & $\begin{array}{l}0.0 \\
0.0 \\
0.0 \\
0.0 \\
0.0 \\
0.0\end{array}$ \\
\hline $\begin{array}{l}\text { Lower Atlantle (PADD Z }) \\
\text { North Carolina } \\
\text { Virginla }\end{array}$ & $\begin{array}{l}0.0 \\
0.0 \\
0.0\end{array}$ & $\begin{array}{l}0.0 \\
0.1 \\
0.0\end{array}$ & $\begin{array}{l}0.0 \\
0.0 \\
0.0\end{array}$ & $\begin{array}{l}0.0 \\
0.0 \\
0.0\end{array}$ & $\begin{array}{l}0.0 \\
0.0 \\
0.1\end{array}$ & $\begin{array}{l}0.0 \\
0.0 \\
0.0\end{array}$ & $\begin{array}{l}0.0 \\
0.0 \\
0.2\end{array}$ & $\begin{array}{l}0.0 \\
0.0 \\
0.0\end{array}$ \\
\hline $\begin{array}{l}\text { Mlawost (PADD II) } \\
\text { Indlana } \\
\text { lowa } \\
\text { Kansas } \\
\text { Michigan } \\
\text { Minnesota } \\
\text { Missouri } \\
\text { North Dakota } \\
\text { Ohlo } \\
\text { South Dakota } \\
\text { Wisconsin }\end{array}$ & $\begin{array}{l}0.1 \\
1.4 \\
0.11 \\
0.0 \\
0.0 \\
0.0 \\
0.0 \\
0.0 \\
0.0 \\
0.0 \\
0.0\end{array}$ & $\begin{array}{l}0.0 \\
0.0 \\
0.0 \\
0.0 \\
0.0 \\
0.1 \\
0.0 \\
0.0 \\
0.0 \\
0.0 \\
0.0\end{array}$ & $\begin{array}{l}0.0 \\
0.0 \\
0.0 \\
0.0 \\
0.0 \\
0.0 \\
0.0 \\
0.0 \\
0.0 \\
0.0 \\
0.0\end{array}$ & $\begin{array}{l}0.0 \\
0.0 \\
0.0 \\
0.0 \\
0.0 \\
0.0 \\
0.0 \\
0.0 \\
0.0 \\
0.0 \\
0.0\end{array}$ & $\begin{array}{l}0.0 \\
0.0 \\
0.0 \\
0.1 \\
0.0 \\
0.0 \\
0.0 \\
0.0 \\
0.0 \\
0.1 \\
0.0\end{array}$ & $\begin{array}{l}0.0 \\
0.0 \\
0.0 \\
0.0 \\
0.0 \\
0.0 \\
0.0 \\
0.0 \\
0.0 \\
0.0 \\
0.0\end{array}$ & $\begin{array}{l}0.1 \\
2.6 \\
0.0 \\
0.0 \\
0.0 \\
0.0 \\
0.0 \\
0.0 \\
0.6 \\
2.5 \\
0.4\end{array}$ & $\begin{array}{l}0.0 \\
0.0 \\
0.0 \\
0.0 \\
0.0 \\
0.0 \\
0.0 \\
0.0 \\
0.0 \\
0.0 \\
0.0\end{array}$ \\
\hline
\end{tabular}

Note: - Due to significant wholesale prices increases between January 4 and January 11, 1993, stemming from low propane stocks in the Midwest, EIA initiated weekly collection of State propane prices to monitor prices at the retail level. - Data in table appear in absolute values.

Source: Based on data collected by State Energy Offices. 


\section{Note 6. Provisions Regarding Confidentiality of Information}

The Office of Legal Counsel of the Department of Justice concluded on March 20, 1991, that the Federal Energy Administration Act requires the Energy Information Administration to provide company-specific data to the Department of Justice, or to any Federal agency when requested for official use, which may include enforcement of Federal law. The information contained on this form may also be made available, upon request, to another component of the Department of Energy (DOE), to any Committee of Congress, the General Accounting Office, or other Congressional agencies authorized by law to receive such information. A court of competent jurisdiction may obtain this information in response to an order.

The information contained on this form will be kept confidential and not disclosed to the public to the extent that it satisfies the criteria for exemption under the Freedom of !nformation Act (FOIA), 5 U.S.C. section 552, the DOE regulations, 10 C.F.R. section 1004.11, implementing the FOIA, and the Trade Secrets ACT, 18 U.S.C. section 1905.

Upon receipt of a request for this information under the FOIA, the DOE shall make a final determination whether the information is exempt from disclosure in accordance with the procedures and criteria provided in the regulations. To assist us in this determination, respondents should demonstrate to the DOE that, for example, their information contains trade secrets or commercial or financial information whose release would be likely to cause substantial harm to their company's competitive position. A letter accompanying the submission that explains (on an element-by-element basis) the reasons why the information would be likely to cause the respondent substantial competitive harm if released to the public would aid in this determination. A new justification does not need to be provided each time information is submitted on the form, if the company has previously submitted a justification for that information and the justification has not changed. 


\section{Glossary}

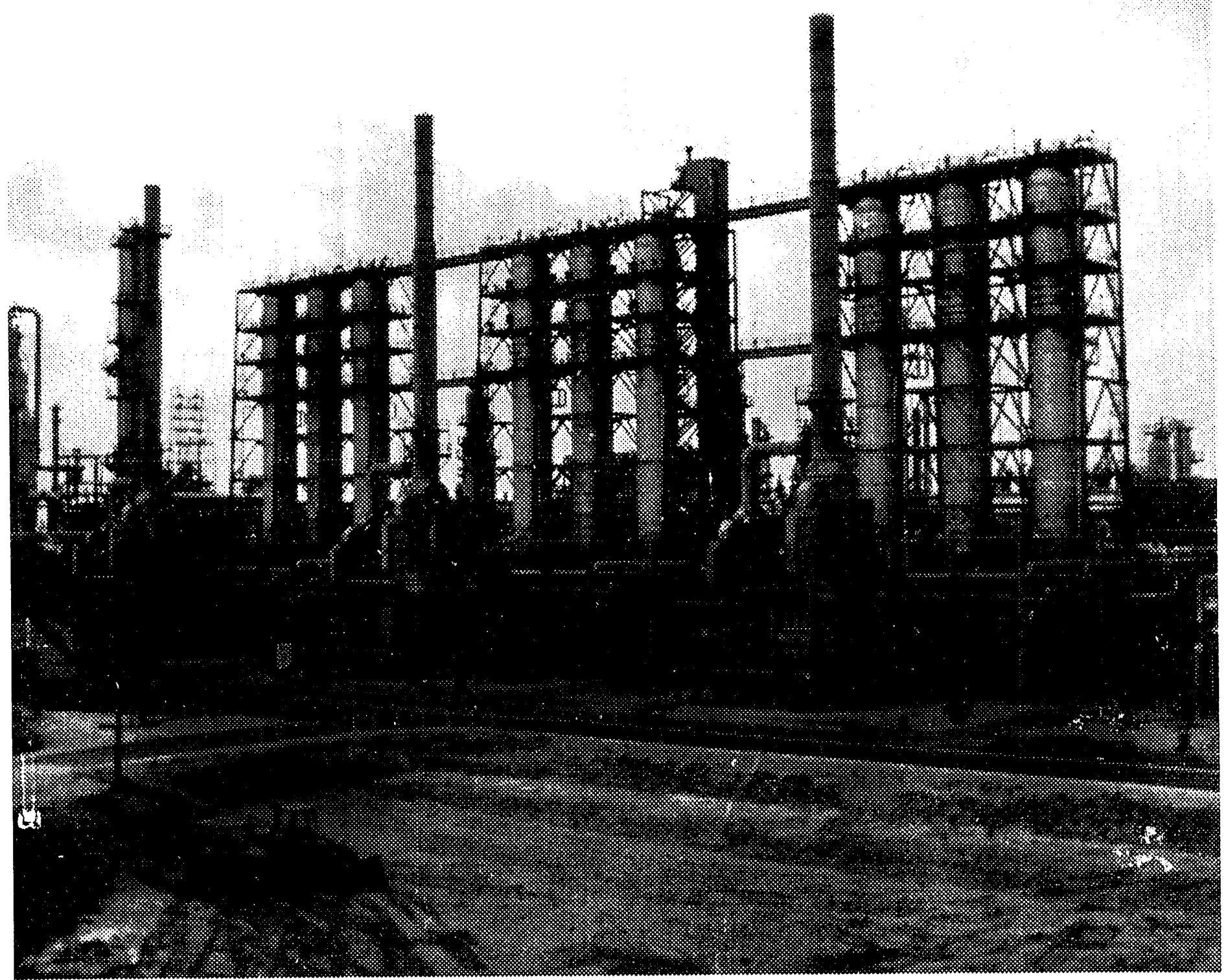




\section{Definitions of Petroleum Products and Other Terms}

Balancing Item. Represents differences between the sum of the components of natural gas supply and the sum of the components of natural gas disposition. These differences may be due to quantities lost or to the effects of data reporting problems. Reporting problems include differences due to the net result of conversions of flow data metered at varying temperatures and pressure bases and converting to a standard temperature and pressure base; the effect of variations in company accounting and billing practices; differences between billing cycle and calendar period time frames; and imbalances resulting from the merger of data reporting systems that vary in scope, format, definitions, and type of respondents.

Barrel. A volumetric unit of measure for crude oil and petrolcum products equivalent to 42 U.S. gallons. This measure is used in most statistical reports.

Bulk Terminal. A facility used primarily for the storage and/or marketing of petroleum products which has a total bulk storage capacity of 50,000 barrels or more and/or receives petroleum products by tanker, barge, or pipeline.

Commercial Consumption. Gas used by nonmanufacturing establishments or agencies primarily engaged in the sale of goods or services. Included are such establishments as hotels, restaurants, wholesale and retail stores and other service enterprises: gas used by establishments engaged in agriculture, forestry, and fisheries; and gas used by local, State, and Federal agencies engaged in nonmanufacturing activities.

Degree-Day Normals. Simple arithmetic averages of monthly or annual degree-days over a long period of time (usually the 30-year period 1961-1990). These may be simple degree-day normals or population-weighted degree-day normals.

Distillate Fuel Oil. A general classification for one of the petroleum fractions produced in conventional distillation operations. It is used primarily for space heating, on-and-off-highway diesel engine fuel (including railroad engine fucl and fuel for agricultural machinery), and electric power generation. Included are products known as No. 1. No. 2, and No. 4 fucl oils; No. 1, No. 2, and No. 4 diesel fucls. Distillate fuel oil is reported in the following sulfur categories: $0.05 \%$ sulfur ind under and Greater than $0.05 \%$ sulfur.

No. 1 Distillate. A petroleum distillate which meets the specifications for No. 1 heating or fuel oil as defined in ASTM D 396 and/or the specifications for No. 1 diesel fuel as defined in ASTM Specification D 975 with distillation temperatures of $420^{\circ} \mathrm{F}$ at the 10 -percent recovery point and $550^{\circ} \mathrm{F}$ at the 90-percent recovery point, and kinematic viscosities between 1.4 and 2.2 centistokes at $100^{\circ} \mathrm{F}$.

No. 2 Distillate. A petroleum distillate which meets the specifications for No. 2 heating or fuel oil as defined in ASTM D 396 and/or the specifications for No. 2 diesel fuel as defined in ASTM Specification D 975 with distillation temperatures of $540^{\circ}$ and $640^{\circ} \mathrm{F}$ at the 90 -percent recovery point, and kinematic viscosities between 2.0 and +.3 centistokes at $100^{\circ} \mathrm{F}$.

No. 4 Fuel Oil. A fuel oil for commercial burner installations not equipped with preheating facilitics. It is used extensively in industrial plants. This grade is a blend of distillate fuel oil and residual fuel oil stocks that conforms to ASTM Specification D396 or Federal Specification VV-F-S15C: with minimum and maximum kinematic viscosities between 5.8 and 26.4 centistokes at $100^{\circ} \mathrm{F}$. Also included is No. +-D. a fuel oil for low and medium-speed diesel engines that conforms to ASTM Specification D975.

Dry Natural Gas Production. Marketed production less extraction loss.

Electric Utility' Consumption. Gas used as fuel in clectric utility plants.

Ending Stocks. Primary stocks of crude oil and petroleum products held in storage as of the end of a specific report period. For the monthly report period this is as of 12 midnight on the last day of the month. For the weckly report period, 7 a.m. cach Friday. Primary stocks include crude oil or petroleum products held in storage at (or in) leases, refineries, natural gas processing plants, pipelines, tank farms, and bulk terminals that can store at least 50,000 barrels of petroleum products or that can receive petroleum products by tanker, barge, or pipeline. Crude oil that is in-transit by water from Alaska, or that is stored on Federal leases or in the Strategic Petroleum Reserve is included. Primary stocks exclude stocks of foreign origin that are held in bonded warehouse storage.

Exports. Shipments of goods from the 50 States and the District of Columbia to foreign countries, Puerto Rico, the Virgin Islands. and other U.S. possessions and territories.

Heating Degree-Days. The number of degrees per day the daily average temperature is below 65 degrees $F$. The daily average temperatire is the mean of the maximum and minimum temperature for a 24-hour period.

Imports. Receipts of goods into the 50 States and the District of Columbia from foreign countries, Pucrto Rico, the Virgin Islands, and other U.S. possessions and territories.

Industrial Consumption. Natural gas used by manufacturing and mining establishments for heat, power, and chemical feedstock.

Liquefied Natural Gas (LNG). Natural gas (primarily methane) that has been liquefied by reducing its temperature to minus 260 degrees Fahrenheit at atmospheric pressure.

Natural Gas. A mixture of hydrocarbon compounds and small quantities of various nonhydrocarbons existing in the gaseous 
phase or in solution with crude oil in natural underground reservoirs at reservoir conditions.

Natural Gas Processing Plant. A gas processing plant is a facility designed (1) to achieve the recovery of natural gas liquids from the stream of natural gas which may or may not have been processed through lease separators and field facilities, and (2) to control the quality of the natural gas to be marketed. Cycling plants are classified as gas processing plants.

Net Production. Petroleum products produced at a refinery, natural gas processing plant, or blending plant. Published production of these products equals production minus input. Negative production will occur when the amount of a product produced during the month is less than the amount of that same product that is reprocessed (input) or reclassified to become another product during the same month.

Pipeline. Crude oil and pruduct pipclines used to transport crude oil and petroleum products respectively, (including interstate, intrastate, and intracompany pipelines) within the 50 States and the District of Columbia.

Population-Weighted Degree-Days. Heating or cooling degree-days weighted by the population of the area in which the degrec-days are recorded. To compute national population-weighted degree-days, the Nation is divided into nine Census regions comprised of from three to eight States which are assigned weights based on the ratio of the population of the region to the total population of the Nation. Degree-day readings for each region are multiplied by the corresponding population weight for each region and these products are then summed to arrive at the national population weighted degrec-day figure.

Product Supplied. Approximately represents consumption of petroleum products because it measures the disappearance of these products from primary sources, i.e., refincries, natural gas processing plants, blending plants, pipelines, and bulk terminals. In general, product supplied of each product in any given period is computed as follows: field production, plus refinery production, plus imports, plus unaccounted for crude oil, (plus net receipts when calculated on a PAD District basis), minus stock change, minus crude oil losses, minus refinery inputs, minus exports.

Propane (C3H8). A normally gascous straight-chain hydrocarbon. It is a colorless paraffinic gas that boils at a temperature of -43.67 degrees $F$. It is extracted from natural gas or refinery gas streams. It includes all products designated in ASTM Specification D1835 and Gas Processors Association Specifications for commercial propane and HD-5 propane.
Propylene (C3H6). An olefinic hydrocarbon recovered from refinery processes or petrochemical processes.

Refinery. An installation that manufactures finished petroleum products from crude oil, unfinished oils, natural gas liquids, other hydrocarbons, and alcohol.

Report Dates. The official report dates for the residential and wholesale price surveys are the first and third Mondays. The official day for the primary stock survey is $7 \mathrm{a} . \mathrm{m}$. on the Friday preceding the report date.

Residential Consumption. Gas used in private dwelling, including apartments, for heating, air conditioning, cooking, water heating, and other household uses.

Residential Heating Oil Price. The price charged for home delivery of No. 2 heating oil, exclusive of any discounts such as those for prompt cash payment. Prices do not include taxes paid by the consumer.

Residential Propane Price. The "bulk keep full" price for home delivery of consumer grade propane intended for use in space heating, cooking, or hot water heaters in residences.

Storage Additions. Volumes of gas injected or otherwise added to underground natural gas reservoirs or liquefied natural gas storage.

Storage Withdrawals. Volumes of gas withdrawn from underground storage or liquefied natural gas storage.

Supplemental Gaseous Fuels Supplies. Synthetic natural gas, propane-air, coke oven gas, refinery gas, biomass gas, air injected for Btu stabilization, and manufactured gas conımingled and distributed with natural gas.

Underground Storage. The storage of natural gas in underground reservoirs at a different location from which it was produced.

Underground Storage Injections. Gas from extraneous sources put into underground storage reservoirs.

Underground Storage Withdrawals. Gas removed from underground storage reservoirs.

United States. For the purpose of this report, the 50 States and the District of Columbia. Data for the Virgin Islands. Puerto Rico, and other U.S. territories are not included in the U.S. Totals.

Wholesale Price. The rack price charged for No. 2 heating oil: that is, the price charged customers who purchase No. 2 heating oil free-on-board at a supplier's terminal and provide their own transportation for the product. 

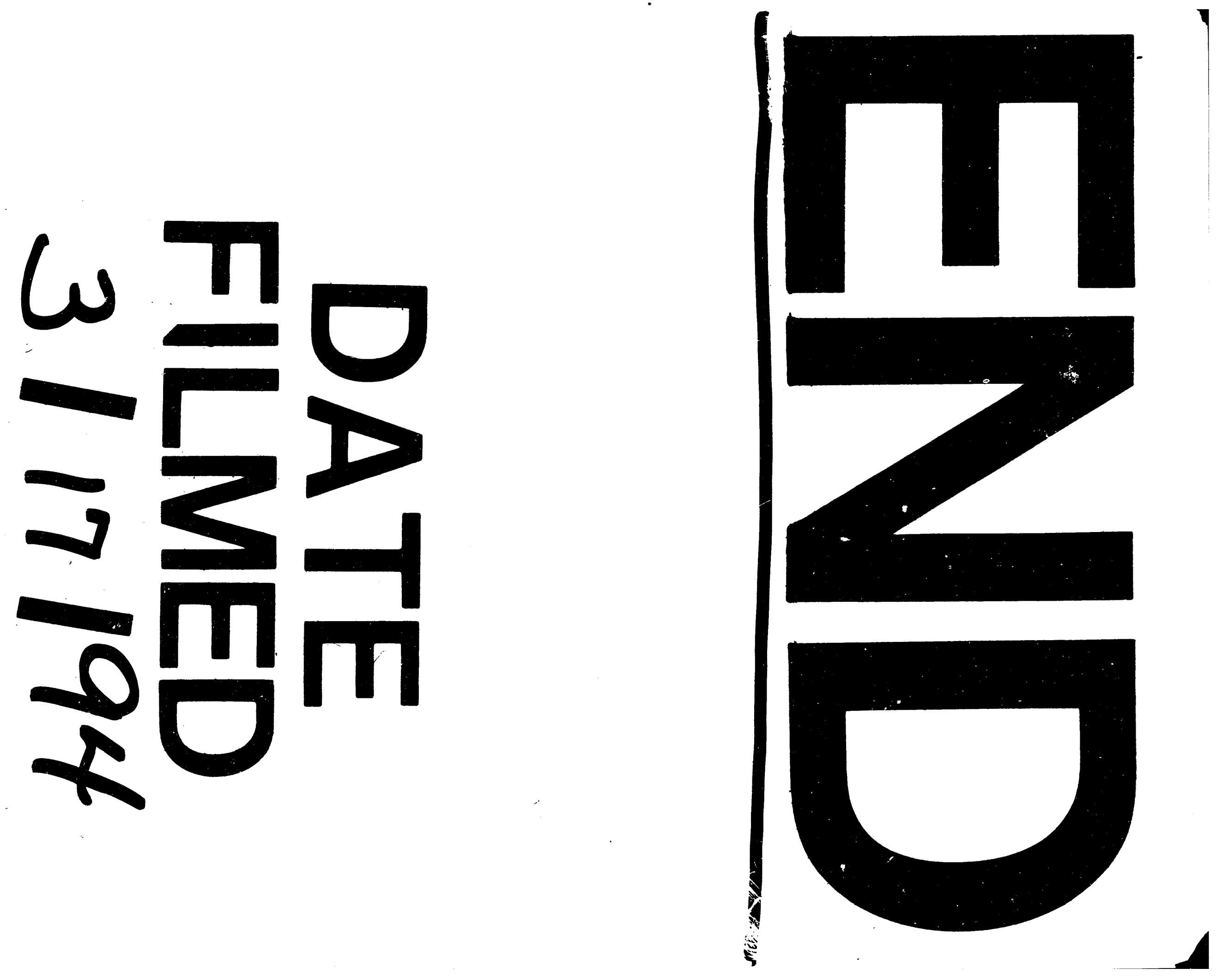
